\title{
Scaffold design and characterisation for osteochondral tissue regeneration
}

\author{
Thesis submitted by \\ Harmony Deplaine \\ to obtain the degree of Dr. Eng. Sci. \\ at the Universitat Politècnica de València \\ Valencia (Spain), 2012
}

Thesis supervisor: Dr. Gloria Gallego Ferrer 

Member of the jury

\section{President}

Dr. Manuel Doblaré Castellano

Universidad de Zaragoza, Spain

\section{Secretary}

Dr. Ana Vallés Lluch

Universitat Politècnica de València, Spain

\section{Members}

Dr. Manuel Salmerón Sánchez

Universitat Politècnica de València, Spain

Dr. Ignacio Ochoa Garrido

Universidad de Zaragoza, Spain

Dr. Iñigo Izal Azcárate

Universidad de Navarra, Spain 

À David, car sans toi ce pas n'aurait pas été possible ... 



\section{Acknowledgements}

This work was financially support from the Spanish Ministry of Science and Innovation through the DPI2007-65601-C03-03 "Diseño y validación experimental de un nuevo concepto de andamiaje bicapa para regeneración de defectos osteocondrales" and its continuity DPI2010-20399-C04-03 "Diseño, construcción y validación de una plataforma biomimética para la evaluación funcional y optimización de constructos de ingeniería tisular de cartilage articular" projects directed by Gloria Gallego Ferrer and the Instituto de Salud Carlos III (ISCIII) through the Centro de Investigación Biomédica en Red en Bioingeniería, Biomateriales y Nanomedicina (CIBER-BBN).

It is a pleasure to thank those who made this thesis possible, all the people of the Centre for Biomaterials and Tissue Engineering. In particular Gloria Gallego Ferrer and José Luis Gómez Ribelles who taught me the values of research. I would like also to thank Manuel Monleón Pradas and José Carlos Rodríguez Hernández for their advices. Thank you Ana Vidaurre Garayo, Roberto García Gomez and, Angel Serrano Aroca for the experimental help.

Thank you Myriam Lebourg for teaching me principles of scaffolding, also to my collegues Miriam, Cristina, Marco, Tatiana, Carmen, Amparo, Pachi, Andrés, Laura.

I am also grateful to the staff of the Microscopy Service of the Universitat Politècnica de València, Manuel Planes Insausti, José Luis Moya López, Mercedes Tabernero Mendoza, and Alicia Nuez Imbernón.

Thank you to Ana Rosalia Villanueva Molines and Samuele Guerzoni (Ulrica Edlund's student) for their work.

I give also my thanks to Daniel Beltrán and Pedro Amorós for purchasing us $\mathrm{SiO}_{2}$ particles.

I am very grateful for the collaboration with the Group of Structural Mechanics and Materials Modelling, of the 
University of Zaragoza, and the Laboratorio de Ortopedia Experimental of the Área de Terapia Cellular of the University of Navarra, in Spain. I would especially mention to Manuel Doblaré, Ignacio Ochoa, Victor Acosta, Iñigo Izal, Felipe Prosper, Gonzalo Mora and Purificación Ripalda.

I would like to thank Colm, Luke and Kat for their help.

I would like to thank my family and very good friends, most especially my mum and David for your love and support. 


\section{Index}

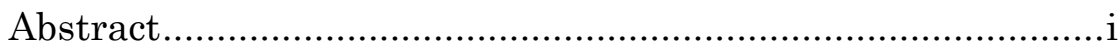

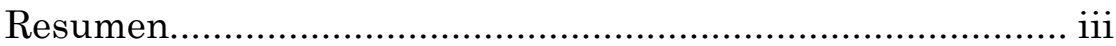

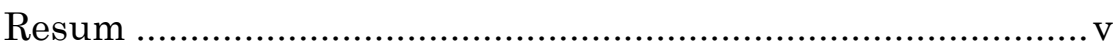

General purposes ............................................................. vii

Specific purposes..................................................................

Chapter 1. Introduction .................................................... 1

$1.1 \quad$ Osteochondral biology............................................. 1

1.1.1 Structure and function of articular cartilage....... 1

1.1.2 Composition, structure and function of subchondral bone ............................................... 6

1.1.3 Cartilage injuries.............................................. 12

1.1.4 Articular cartilage repair conventional therapies. 14

1.1.5 Tissue engineering (TE) approach for the regeneration of articular cartilage .................... 17

1.1.6 TE approach for the regeneration of bone..........21

1.2 Osteochondral tissue engineering ...........................26

1.2.1 Purpose of the strategy ..................................26

1.2.2 Layered scaffolds for osteochondral TE: an overview .................................................... 27

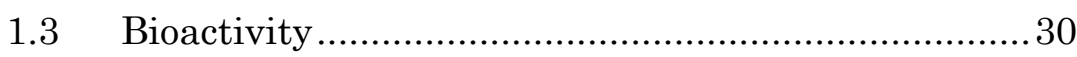

1.3.1 In vivo bioactivity ............................................ 30

1.3.2 Bioactive materials and mechanism of apatite formation......................................................... 33

1.4 Synthetic polymers biodegradation.......................... 36

1.4.1 Mechanisms of biodegradation.......................... 36

1.4.2 Effect of different parameters on the rate of biodegradation ................................................. 38

1.5 Poly(L-lactic acid) and poly(L-lactic acid)/ inorganic composite scaffolds ................................................ 39

1.5.1 Main properties of poly(L-lactic acid)................ 39

1.5.2 An overview to poly(L-lactic acid) scaffold fabrication techniques ...................................... 41 
1.5.3 Bioactive poly(L-lactic acid)/inorganic composites

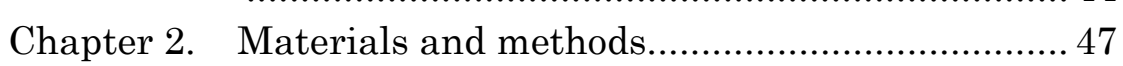

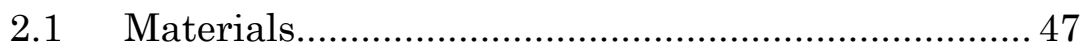

2.1.1 Micro and macro porous scaffolds..................... 47

2.1.2 Hybrid scaffolds PLLA/bioactive particles......... 50

2.1.3 Bioactivity test .............................................. 53

2.1.4 Oxygen plasma treatment................................ 54

2.1.5 Sonication ...................................................... 54

2.1.1 Static degradation ......................................... 54

2.1.2 Fatigue essay .............................................. 55

2.1.3 Biconstruct fabrication: bilayered scaffold for osteochondral regeneration .............................. 56

2.2 Characterization techniques .................................. 57

2.2.1 Scanning Electron Microscopy (SEM)............... 57

2.2.2 Elemental analysis (EDS) ............................... 58

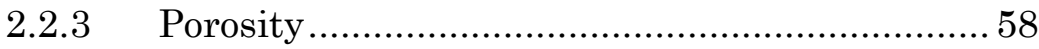

2.2.4 Compressive test ......................................... 59

2.2.5 Dynamic mechanical spectroscopy (DMS) .........61 61

2.2.6 Differential scanning calorimetry (DSC) ...........61 61

2.2.7 Gel permeation chromatography (GPC) ........... 61

2.2.8 Thermogravimetric analysis (TGA) ..................6 61

2.2.9 Calcination experiments ................................62 62

2.2.10 Fourier transform infrared analysis (FTIR) ...... 62

Chapter 3. Results .......................................................... 63

3.1 Scaffolds fabrication: Processing, morphology and

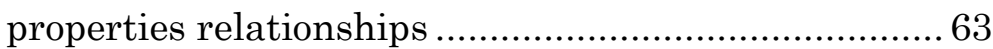

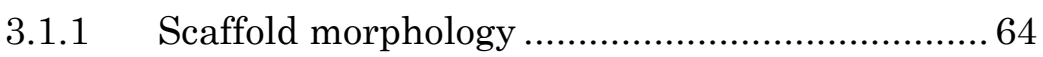

3.1.2 Porosity ....................................................... 71

3.1.3 Mechanical properties ...................................... 73

3.1.4 Calorimetric properties ................................... 78

3.2 Degradation experiments ..................................... 83

3.2.1 Static degradation .......................................... 84

3.2.2 Fatigue essay ............................................... 102

3.3 Reinforcement and bioactivity of the material ....... 117 
3.3.1 2D hybrid structure ....................................... 118

3.3.2 3D hybrid structure ........................................ 134

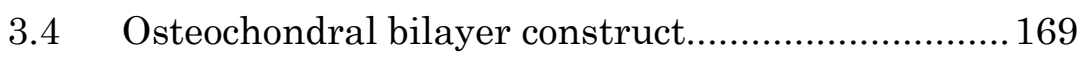

Chapter 4. Discussion .................................................. 171

4.1 Scaffolds fabrication: Processing-morphology-properties relationships ...................................................... 171

4.1.1 Effect on the scaffold properties...................... 171

4.1.2 Selection of a composition ............................... 176

4.1.3 Partial conclusions.......................................... 176

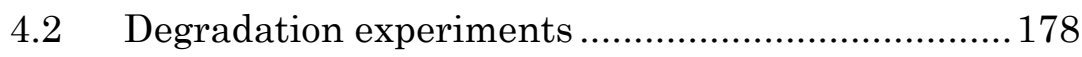

4.2.1 Partial conclusions......................................... 180

4.3 Reinforcement and bioactivity of the material ....... 181

4.3.1 Effect of the filler content on the membrane's properties ...................................................... 181

4.3.2 Effect of the filler content on the scaffold's properties ...................................................... 184

4.3.3 Effect of filler type ........................................ 187

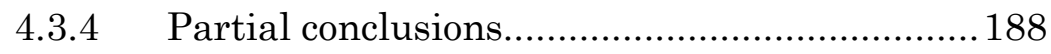

4.4 Bilayer construct ................................................ 191

Chapter 5. Conclusions ...................................................... 193

Chapter 6. Appendix. In vitro and in vivo biological response

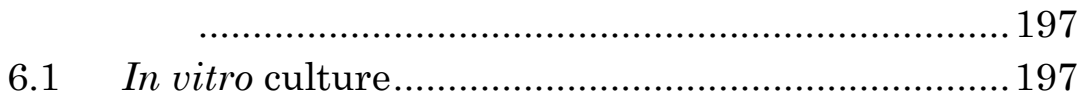

6.2 Implantation ..................................................... 204

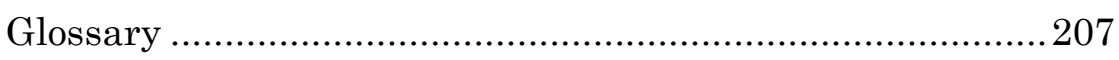

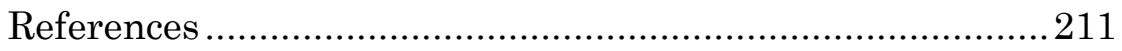





\section{Abstract}

The main purpose of this thesis is the design of a polymeric bilayer scaffold with porous architecture for osteochondral regeneration. The material used for the construct fabrication was poly(L-lactic acid), a biodegradable polymer of the family of polyesters. One of the layers of the scaffold was designed to attend the regeneration of articular cartilage. The other layer serves as anchorage to subcondral bone, one difference with the previous layer is in the mechanical properties and the bioactivity. This behavior was obtained by combination of poly(L-lactic acid) with inorganic nanoparticles. Both layers are joined to each other by a thin layer of nonporous material, that avoids the flow of cells from one part to the other one.

For this aim, first, scaffolds with different porous morphology were fabricated varying parameters of fabrication such as the polymer concentration and the polymer / porogen ratio, in order to obtain the more suitable structure for the regeneration of both tissues. Then they were evaluated in regard to their mechanical and physical-chemical properties, in order to select the parameters of fabrication which lead to greater results.

In tissue regeneration it is essential to know how the implant properties will vary with time when implanted and when its degradation starts. Consequently we considered opportune studying in vitro degradation of the material in different conditions. The degradation study was realised by static degradation during $6,12,18,24$ weeks and 1 year and fatigue essay during 1, 2, 4 and 6 weeks. Then the mechanical properties and physical-chemical properties were evaluated after the different times of degradation.

Later, and to increase the mehanical properties and bioactivity of the bone construct, different contents of bioactive 
nanoparticles of hydroxyapatite and silica were incorporated to the material. The influence of the incorporation of nanoparticles on the mechanical and physical-chemical properties was evaluated. Special emphasis was made in evaluating the increase of the bioactive potential provided by the inorganic filler. The bioactivity was evaluated thanks to the in vitro test consisting in studying the capacity of the material in nucleating biomimetcic hydroxyapatite crystals on its surface, while immersed in simulated body fluid during different times.

These studies allowed selecting the optimal parameters of synthesis for the bilayer osteochondral construct, which was fabricated for in vivo evaluation in animals. 


\section{Resumen}

El objetivo principal de esta tesis doctoral es el diseño de un andamio polimérico bicapa macroporoso para la regeneración del complejo osteocondral. El material empleado para la fabricación del constructo ha sido el ácido poli(L-láctico), un polímero biodegradable de la familia de los poliésteres. Una de las capas del andamio ha sido diseñada para asistir la regeneración del cartílago articular. La otra capa sirve de anclaje al hueso subcondral, y se diferencia de la anterior en sus propiedades mecánicas y bioactividad. Este comportamiento ha sido logrado por combinación del ácido poli(L-láctico) con nanopartículas inorgánicas. Ambas capas están unidas entre sí por una fina capa de material no poroso que evita el flujo de células de una parte a otra del constructo. Para lograr este objetivo se realizó un primer estudio de diseño variando la morfología de los andamios hasta obtener aquella arquitectura más adecuada para la regeneración de ambos tejidos. Se varió parámetros de síntesis tales como la concentración de polímero y el ratio entre polímero y porógeno. Los andamios fueron evaluados mecánica y fisicoquímicamente y se seleccionó los parámetros de síntesis del ácido poli(L-láctico) que dieron mejores resultados.

En la regeneración del tejido es esencial conocer cómo variarán las propiedades del material una vez sea implantado y comience su degradación. Por lo tanto, fue considerado oportuno realizar un estudio de degradación del material in vitro en diversas condiciones. El estudio de la degradación fue realizado en condiciones estáticas durante $6,12,18,24$ semanas y 1 año y en condiciones dinámicas durante 1, 2, 4 y 6 semanas. Se evaluó tanto las características mecánicas como las fisicoquímicas tras los diversos tiempos de la degradación. 
Posteriormente, y para aumentar las características mecánicas y la bioactividad del anclaje óseo, se incorporó distintas cantidades de nanopartículas inorgánicas de hidroxiapatita y sílice a los andamios. La influencia de la cantidad de nanopartículas incorporadas y su naturaleza en las características mecánicas y fisicoquímicas de los materiales fueron estudiadas. Se hizo especial hincapié en la mejora del potencial bioactivo proporcionado por la carga inorgánica. Para ello se realizó ensayos de bioactividad in vitro consistentes en estudiar la capacidad del material de nuclear hidroxiapatita biomimética en su superficie cuando es sumergido en fluido biológico simulado durante distintos tiempos.

Estos estudios permitieron elegir los parámetros de síntesis óptimos para el andamiaje bicapa osteocondral, que fue fabricado para su evaluación in vivo en animales de experimentación. 


\section{Resum}

L'objectiu principal d'aquesta tesi doctoral és el disseny d'un constructe polimèric bicapa macroporós (scaffold) per a la regeneració del teixit osteocondral. El material emprat per a la fabricació del constructe ha segut l'àcid poli(L-làctic), un polímer biodegradable de la família dels polièsters. Una de les capes del constructe ha segut dissenyada per a assistir la regeneració del cartílag articular. L’altra capa serveix d'ancoratge a l'os subcondral, i es diferencia de l'anterior en les seves propietats mecàniques i bioactivitat. Aquest comportament ha segut atansat amb la combinació de l'àcid poli(L-làctic) amb nanopartículas inorgàniques. Ambdues capes estan unides entre sí per una fina capa de material no porós, que evita el flux de cèl•lules d'una part a l'altra del constructe.

Per tal d'obtenir aquest objectiu es realitzà un primer estudi de disseny variant la morfologia dels scaffolds fins a obtenir aquella arquitectura més adequada per a la regeneració dels dos teixits. Es varià paràmetres de síntesi tals com la concentració de polímer i el ràtio entre polímer i porogen. Els scaffods van ser avaluats mecànica i fisicoquímicament i se seleccionà els paràmetres de síntesi que donaren els millors resultats.

En la regeneració del teixit és essencial conèixer com varien les propietats del material una vegada és implantat i comenci la seva degradació. Per tant, es considerà oportú realitzar un estudi de degradació del material in vitro en diverses condicions. L'estudi de degradació es realitzà en condicions estàtiques durant $6,12,18,24$ setmanes $i 1$ any $i$ en condicions dinàmiques durant $1,2,4$ i 6 setmanes. Es va avaluar tant les característiques mecàniques com fisicoquímiques després de diversos temps de degradació. 
Posteriorment, i per tal d'augmentar les propietats mecàniques i la bioactivitat de l'ancoratge ossi, s'incorporà distintes quantitats de nanopartícules inorgàniques d'hidroxiapatita i sílice als scaffolds. La influència de la quantitat de nanopartícules incorporades i la seva naturalesa en les propietats mecàniques i fisicoquímiques dels materials van ser estudiades. Es posà l'accent en la millora del potencial bioactiu proporcionat per la càrrega inorgànica. Per a això, es realitzà assajos de bioactivitat in vitro consistents en estudiar la capacitat del material de nuclear hidroxiapatita biomimètica a la superfície quan és submergit en fluid biològic simulat durant diversos temps.

Aquestos estudis permeteren escollir els paràmetres de síntesi òptims per al constructe bicapa osteocondral, que va ser fabricat per a la seva avaluació in vivo en animals d'experimentació. 


\section{General purposes}

This thesis focuses on the design of a bilayer scaffold for osteochondral complex regeneration. The first layer presents similar characteristics to articular cartilage. In this layer mesenchymal cells will be cultivated and pre-differentiated to chondrocytes in vitro to promote cartilage regeneration when implanted in vivo. The second layer presents the bioactivity necessary to favour the anchorage to subchondral bone and mechanical properties similar to the surrounding bone. The two layers will be separated by an impermeable layer in order to avoid vascularization and migration of cells in the cartilage layer from the bone layer. The material selected for the design was poly(L-lactic acid), a biodegradable polymer approved by the FDA for medical devices.

\section{Specific purposes}

- Design of porous scaffolds with interconnected pores and a double porosity, micrometric and macrometric, able to reproduce the mechanical properties of artilcular cartilage and subchondral bone.

- Evaluate the relationship between the properties of the scaffolds (porosity, micro and macrostructure, mechanical and other physicochemical properties) and the fabrication parameters, polymer/solvent ratio and polymer/porogen ratio.

- Evaluate the effect of static and dynamic degradation on the structure, mechanical and physical-chemical properties of the scaffolds. 
- Modify the scaffolds to increase their bioactivity and mechanical properties for the bone anchorage layer.

- Assembly of the cartilage and bone scaffolds into an osteochondral construct.

- The biological response of the designed scaffolds was not a direct objective to this thesis but thanks to the collaboration with the Laboratorio de Ortopedia Experimental and Área de Terapia Celular of the Universidad de Navarra and the Group of Structural Mechanics and Materials Modelling, of the University of Zaragoza, it was possible to test samples in in vitro and in vivo. Some of the results are shown in the appendix of this thesis. 


\section{Chapter 1. Introduction}

\subsection{OSTEOCHONDRAL BIOLOGY}

\subsubsection{Structure and function of articular cartilage}

Cartilage is a highly specialized, avascular, aneural, connective tissue found throughout the body. The primary function of this tissue in all locations is to provide structural support for surrounding tissues. There are four major types of cartilage each distinguished by specific constitutive or organizational components: hyaline or articular cartilage, elastic cartilage, and fibrocartilage.

Elastic cartilage or yellow cartilage is a type of cartilage present in the outer ear, larynx and epiglottis. It contains elastic fibre networks and collagen fibres of type II and few ones of type IX, X and XI. The principal protein is elastin. Elastic cartilage is histologically similar to hyaline cartilage but contains many yellow elastic fibres lying in a solid matrix. These fibres give elastic cartilage great flexibility so that it is able to withstand repeated bending. Chondrocytes, which are the cartilage-specific cells, lie between the fibres.

Fibrocartilage owes its flexibility and toughness to the former of these constituents, and its elasticity to the latter. Fibrocartilage is the only type of cartilage that contains type I collagen in addition to the normal type II. Chondrocytes are also the fibrocartilage cells. Fibrocartilage is found in the pubic symphysis, the annulus fibrosus of intervertebral discs, meniscus and the temporomandibular joint (1).

Articular cartilage is the most prevalent type in the body and is distinguished by shiny white appearance. It is a tissue of mesenchymal origin that covers the articulating surfaces of bones. Articular cartilage has different functions: it spreads 
the applied load onto the subchondral bone; it provides the articular surfaces with low friction and lubrication; it is also responsible for the mechanism of shock-absorption.

Hyaline cartilage is a flexible tissue consisting of cartilage cells, chondrocytes, scattered through a woven network of collagen fibres and proteoglycans distributed within the extracellular matrix (ECM) containing $60-80 \%$ of water (2).

In humans, chondrocytes represent only about $1 \%$ of the volume of hyaline cartilage but are essential since it is these cells that replace degraded matrix molecules to maintain the correct size and mechanical properties of the tissue. Thus, chondrocytes produce and maintain components of ECM including collagen, glycoaminoglycans (GAGs) and proteoglycans (3).

Collagen types II, VI, IX, X and XI are found in articular cartilage, although type II accounts for $90-95 \%$ of the collagen in the matrix. Type II collagen has a high amount of bound carbohydrate groups, allowing more interaction with water than some other types. Collagen types IX and XI, along with type II, form fibrils that interweave to form a mesh. This organization provides tensile strength as well as physically entrapment of other macromolecules. Type $\mathrm{X}$ is found only near the areas of the matrix that are calcified.

The ECM in hyaline cartilage contains three kinds of GAGs: hyaluronan, chondroitin sulphate and keratan sulphate. The hyaluronan molecule is important in the structure of the ECM as it forms a linear aggregate and is interwoven with the network of collagen fibrils. A proteoglycan monomer consists of a core protein joined by GAGs. The most important proteoglycan monomer in the ECM is aggrecan, which is joined by chondroitin sulphate and keratan sulphate molecules. Proteoglycans are hydrophilic; this property results in the ability of hyaline cartilage to retain water which is essential to its proper function. The principal role of articular cartilage is to efficiently transmit weight loads to the 
underlying bone. Keratan sulfate and chondroitin sulfate on the GAGs carry a negative charge. This negative charge creates a high affinity for water that helps cartilage to resist compressive loads and causes the aggrecans to repel one another, resulting in maximal volume expansion. The flow of water through charged regions of the proteoglycan-rich matrix generates piezoelectric charges that further modulate the rate of water flow contributing to the viscoelastic behaviour of articular cartilage. In addition, there is evidence that electric and electromagnetic fields can produce a sustained upregulation of growth factors in articular cartilage (4).

The proteoglycan aggregate structure is presented in Figure $1-1$.

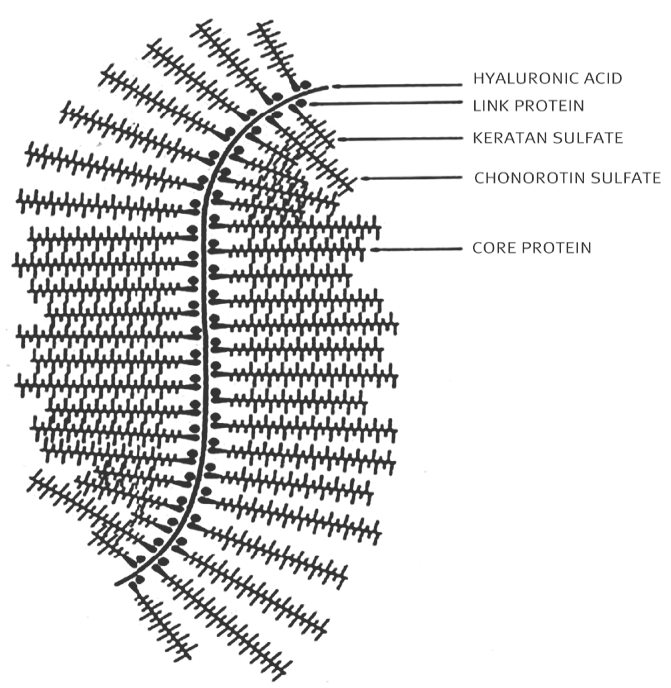

Figure 1-1: Proteoglycan aggregate structure. Reproduced from (4).

As represented in Figure 1-2, articular cartilage can be divided into four zones: superficial, transitional, middle and calcified; based on differences in matrix morphology and biochemistry. The superficial zone is the thinnest zone and is made of two distinct layers. An acellular sheet of 
predominantly collagen fibers covers the joint. Below this, the second layer is composed of flattened chondrocytes with their long axes parallel to the articular surface. The ECM in this area has more collagen and less proteoglycan than the other zones. There are also large amounts of fibronectin and water. This combination of molecules imparts more tensile strength to this area of the matrix, which could be useful in resisting shear stress from the articulating surfaces. Occupying more volume than the superficial zone, the transitional zone includes chondrocytes that are spherical and contain synthetic organelles such as the endoplasmic reticulum, Golgi bodies and mitochondria. The ECM in this area has larger collagen fibrils, more proteoglycan and less collagen and water than in the previous zone. The interterritorial fibrils are aligned obliquely or randomly to the articular surface, as opposed to parallel in the superficial zone. The middle zone is usually the biggest and has the largest diameter collagen fibrils, the most proteoglycan and the least water. The cells are rounded, like in the transitional zone, but are stacked in columns perpendicular to the articulating surface. The territorial region of the matrix encloses each column. These cells show high synthetic activity, ten times that of superficial zone chondrocytes. Also, in this area, the orientation of the interterritorial fibres changes again so that they are now aligned perpendicular to the joint surface. The fibres extend into the tidemark, a basophilic line of unknown composition that indicates the beginning of calcified tissue. The thin calcified cartilage zone lies closest to the subchondral bone and acts as a transition from soft hyaline cartilage to bone. Significant shear stress can be produced in this area due to the interface between the soft cartilage and much stiffer bone. The chondrocytes here are smaller, with almost no endoplasmic reticulum. In some places, they seem to be completely surrounded by calcified ECM, indicating that they have very little metabolic activity (5). 


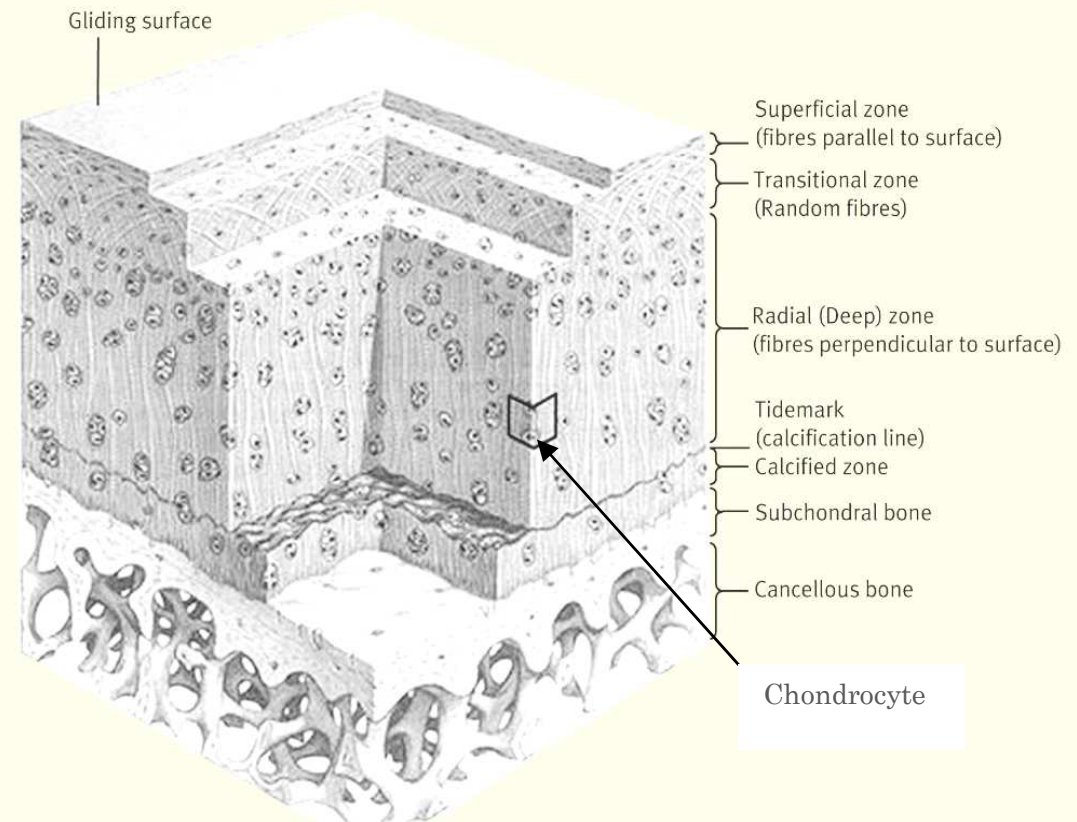

Figure 1-2: Three dimensional organisation of articular cartilage. Produced from (4).

The complex network formed by proteoglycans and type II collagen, provides resistance to a compressive stress and high elasticity to the articular cartilage. The equilibrium modulus of articular cartilage in compression can range from 0.05 to 0.8 $\mathrm{MPa}$. The network confers to the matrix porosity between 65 and $80 \%$, with pores of 60 Angstroms in normal conditions, which could be reduced up to 20 Angstroms when cartilage is compressed. When a load is applied, the water flows very slowly out of the tissue. This slow flow is due to two factors, the low porosity and the presence of the negative charges contained in the GAGs molecules, which exert a strong resistance to a volume reduction. In these conditions the load bearing capacity is influenced mainly by liquid. The uncompressible water sustains the compressive stresses, ultimately protecting the solid components of the cartilage 
matrix, which appears only partially involved in the biomechanical response. If cartilage is damaged the water flows out rapidly from the ECM involving significantly the solid component of the tissue in the biomechanical response. This may lead to a rapid deterioration of the whole cartilaginous tissue (3). Articular cartilage can reach a thickness of up to $7-8 \mathrm{~mm}$ in human knee joint (6).

\subsubsection{Composition, structure and function of subchondral bone}

Bones are rigid organs that constitute part of the endoskeleton of vertebrates. Bone tissue is a type of dense connective tissue. The extra cellular matrix of bone comprises of both a mineralized inorganic component and an organic one. It is the mineralized osseous tissue that gives it rigidity and a honeycomb-like three-dimensional internal structure.

The adult human skeleton is composed of $80 \%$ cortical bone, also called compact bone, and $20 \%$ trabecular bone, also called cancelleous bone. In Figure 1-3 is presented the structure of bone. 

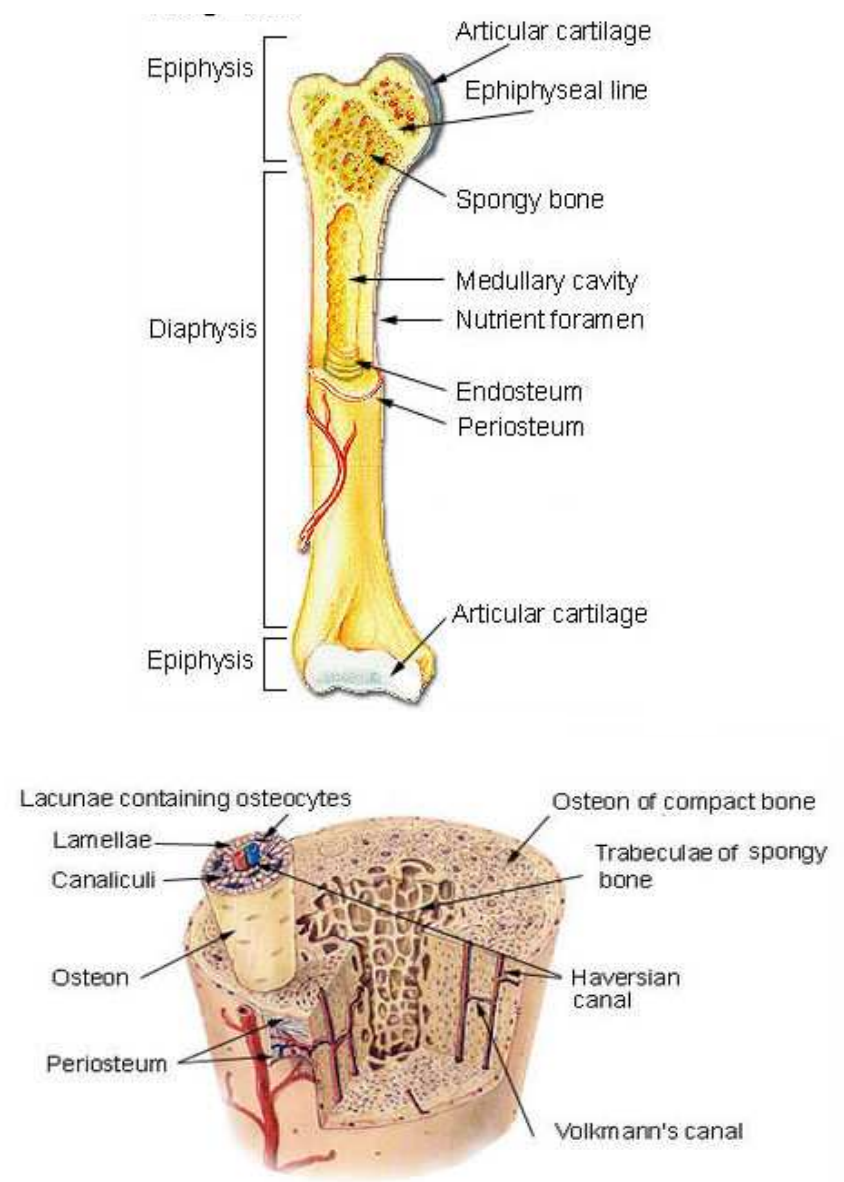

Figure 1-3: Hieralgical structure of bone.

The hard outer layer of bones is composed of compact bone tissue, so-called due to its minimal gaps and spaces. Filling the interior of the bone is the trabecular bone tissue, an open cell porous network which is composed of a network of rodand plate-like elements that make the overall organ lighter and allow room for blood vessels and marrow. Trabecular bone accounts for the remaining $20 \%$ of total bone mass but has nearly ten times the surface area of compact bone. Its porosity is $30-90 \%$. Both cortical and trabecular bone are composed of osteons. Cortical osteons are called Haversian systems. 
Haversian systems are cylindrical in shape, are approximately $400 \mathrm{~mm}$ long and $200 \mathrm{~mm}$ wide at their base, and form a branching network within the cortical bone. The periosteum is a fibrous connective tissue that surrounds the outer cortical surface of bone, except at joints where bone is lined by articular cartilage. The periosteum is tightly attached to the outer cortical surface of bone by thick collagenous fibers, called Sharpeys' fibers, which extend into underlying bone tissue. The endosteum is a membranous structure covering the inner surface of cortical bone, trabecular bone, and the blood vessel canals (Volkman's canals) present in bone.

At the joint, the epiphysis is covered with articular cartilage; below that covering is a zone similar to the epiphyseal plate, known as subchondral bone.

Bone is composed of cells such as osteoblasts and osteoclasts which control the formation or the destruction of tissue. Osteoblasts are located on the surface of bone or osteoid, and they are responsible for synthesizing the organic components of the bone matrix, including type I collagen, proteoglycans, and glycoproteins. Osteoblasts also synthesize the enzyme alkaline phosphatase, which is needed locally for the mineralization of osteoid, the matrix produced by osteoblasts. Osteoblasts contact their neighbouring osteoblasts cytoplasmically. These cells have polarity with the cytoplasm toward the bone and the nuclei at the opposite end away from the bone. Osteoblasts do not divide. They give rise to osteocytes, remain as osteoblasts, or return to the state of osteoprogenitor cells from which they derived $(7,8,9)$.

An osteoblast becomes an osteocyte when the cell is encased by osteoid matrix that it synthesizes itself. Lacunae and canaliculi form around the osteocyte and its cytoplasmic processes, respectively (10). Thus, an osteocyte lies in its own lacuna and contacts its neighbouring osteocytes cytoplasmically through canaliculi. 
Adjacent cells make contact via gap junctions, maintaining the vitality of osteocytes by passing nutrients and metabolites between blood vessels and distant osteocytes. Although osteocytes have reduced synthetic activity and are not capable of mitotic division, they are actively involved with the maintenance of the bony matrix. Some of the osteocytes die during remodelling, but most probably return to the state of osteoprogenitor cells or persist as osteocytes for a long time.

The osteoclasts are probably derived from a monocyticmacrophage system and are responsible for bone resorption, and are the only cells to resorb mineralized bone matrix.

Similarly to cartilage, bone cells are part of the matrix. Bone matrix consists of organic and inorganic components. The association of organic and inorganic substances gives bone its hardness and resistance. The organic component is composed of collagen fibers with predominately type I collagen (95\%) and amorphous material, including glycosaminoglycan (chondroitin sulphate) that are associated with proteins, with amino acid content rich in glycine, leucine, glutamate and aspartate. Osteoid is uncalcified organic matrix. Inorganic matter represents about $50 \%$ of the dry weight of bone matrix, composed of abundant calcium and phosphorus, as well as smaller amounts of bicarbonate, citrate, magnesium, potassium, and sodium. Calcium forms hydroxyapatite (HAp) crystals with phosphorus but is also present in an amorphous form. The ratio between calcium and phosphate is a way to characterise the type of hydroxyapatite, stoichiometric hydroxyapatite $\left(\mathrm{Ca}_{10}\left(\mathrm{PO}_{4}\right)_{6}(\mathrm{OH})_{2}\right)$ has a calcium phosphate ratio of 1.67 , and physiological $\mathrm{HAp}$ a $\mathrm{Ca} / \mathrm{P}$ ratio of 1.65 . Bone hydroxyapatite crystals are very small, 200 Angstroms. Matrix maturation is associated with expression of alkaline phosphatase and several noncollagenous proteins, including osteocalcin, osteopontin and bone sialoprotein. It is thought that these calcium and phosphate binding proteins help 
regulate ordered deposition of mineral by regulating the amount and size of hydroxyapatite crystals formed.

Bone mineral provides mechanical rigidity and load-bearing strength to bone, whereas the organic matrix provides elasticity and flexibility. Bone mineral is initially deposited in "hole" zones between the ends of collagen fibrils. This process may be facilitated by extracellular matrix vesicles in bone. Matrix extracellular vesicles are synthesized by chondrocytes and osteoblasts and serve as protected microenvironments in which calcium and phosphate concentrations can increase sufficiently to precipitate crystal formation. The extracellular fluid is not normally supersaturated with hydroxyapatite, so hydroxyapatite does not spontaneously precipitate. Matrix extracellular vesicles contain a nucleational core that is composed of proteins and a complex of acidic phospholipids, calcium and inorganic phosphate that is sufficient to precipitate hydroxyapatite crystals. It is not yet certain how matrix extracellular vesicles contribute to mineralization points at the ends of collagen fibrils, because the vesicles apparently are not directly targeted to the ends of fibrils. There is no evidence that noncrystalline calcium phosphate clusters (amorphous calcium phosphate) form in bone before they are converted to hydroxyapatite. As bone matures, hydroxyapatite crystals enlarge and reduce their level of impurities. Crystal enlargement occurs both by crystal growth and by aggregation. Confirmed mineralization promoters (nucleators) include dentin matrix protein 1 and bone sialoprotein. Type I collagen is not a bone mineralization promoter. Phosphoprotein kinases and alkaline phosphatase regulate the mineralization process. Bone alkaline phosphatase may increase local phosphorus concentrations, remove phosphate-containing inhibitors of hydroxyapatite crystal growth, or modify phosphoproteins to control their ability to act as nucleators. Vitamin D plays an indirect role in stimulating mineralization of unmineralized bone matrix. 
After absorption or skin production of vitamin D, the liver synthesizes 25-hydroxyvitamin $\mathrm{D}$ and the kidneys subsequently produce biologically active 1.25dihydroxyvitamin D [1.25- $(\mathrm{OH}) 2 \mathrm{D}]$. Serum $1.25-(\mathrm{OH}) 2 \mathrm{D}$ is responsible for maintaining serum calcium and phosphorus in adequate concentrations to allow passive mineralization of unmineralized bone matrix (11).

Bone remodelling is the process by which bone is renewed to maintain bone strength and mineral homeostasis. Remodelling involves continuous removal of discrete packets of old bone, replacement of these packets with newly synthesized proteinaceous matrix and subsequent mineralization of the matrix to form new bone. Remodelling begins before birth and continues until death. The bone remodelling unit is composed of a tightly coupled group of osteoclasts and osteoblasts that sequentially carry out resorption of old bone and formation of new bone. The remodelling cycle is composed of four sequential phases. Activation precedes resorption, which precedes formation. Remodelling sites are thought to develop mostly in a random manner. Activation involves recruitment and activation of mononuclear monocyte-macrophage osteoclast precursors from the circulation, lifting of the endosteum that contains the lining cells of the bone surface, and fusion of multiple mononuclear cells to form multinucleated preosteoclasts. Osteoclast-mediated bone resorption takes only approximately 2 to 4 weeks during each remodelling cycle. Resorbing osteoclasts secrete hydrogen ions via $\mathrm{H}^{+}$-ATPase proton pumps and chloride channels in their cell membranes into the resorbing compartment to lower the $\mathrm{pH}$ within the boneresorbing compartment to as low as 4.5 , which helps mobilize bone mineral. Resorbing osteoclasts secrete tartrate-resistant acid phosphatase, cathepsin $\mathrm{K}$, matrix metalloproteinase 9, and gelatinase from cytoplasmic lysosomes to digest the organic matrix. Resorption phase is completed by mononuclear 
cells after the multinucleated osteoclasts undergo apoptosis. At the completion of bone resorption, cavities contain a variety of mononuclear cells, including monocytes, osteocytes released from bone matrix and preosteoblasts recruited to begin new bone formation. The coupling signals linking the end of bone resorption to the beginning of bone formation are as yet unknown. Bone formation takes approximately 4 to 6 months to complete. Osteoblasts synthesize new collagenous organic matrix and regulate mineralization of matrix by releasing small, membrane-bound matrix vesicles that concentrate calcium and phosphate and enzymatically destroy mineralization inhibitors such as pyrophosphate or proteoglycans. Osteoblasts surrounded by and buried within matrix become osteocytes. At the completion of bone formation, approximately 50 to $70 \%$ of osteoblasts undergo apoptosis, with the balance becoming osteocytes or bone-lining cells. Bone-lining cells may regulate influx and efflux of mineral ions into and out of bone extracellular fluid, thereby serving as a blood bone barrier, but retain the ability to redifferentiate into osteoblasts upon exposure to parathyroid hormone or mechanical forces (11).

\subsubsection{Cartilage injuries}

Articular cartilage is a metabolically active tissue, but it has a poor intrinsic healing potential when damaged. It is able to repair but not to regenerate itself. A reparative process may replace the damaged cartilage by a tissue that fails. The cartilage response to traumas is limited for two reasons: first, cartilage is an avascularised tissue and therefore, when a cartilaginous lesion occurs, the blood clot formation cannot take place and the inflammation response, often accompanied by the migration of the undifferentiated cells to the lesion site, is absent. Second, there is a lack of undifferentiated cells. The number of the chondrocytes and their synthetic properties 
further decline with age, making the repair process further difficult in adult and old patients (3).

Globally cartilage injuries can be classified by three main types: matrix disruption, partial thickness defects and full thickness defects. In the case of matrix disruption ECM is damaged, but if the injury is not extreme, the remaining viable chondrocytes will increase their synthetic activity to repair the tissue. Partial thickness defects demonstrate disruption of the cartilage surface (fissures, etc.) but this does not extend to the subchondral bone. Immediately following the injury, nearby cells begin to proliferate. Full thickness defects transverses the entire cartilage thickness and penetrates the subchondral bone. In this case, the defect is filled with a fibrin clot and a classic wound healing response occurs. With this type of injury, progenitor cells from the bone marrow can migrate to fill the defect (5).

One of the most common diseases of cartilage is osteoarthritis (OA), also called degenerative arthritis, involving the degradation of joints including articular cartilage and subchondral bone. It has been studied that cartilage defects may result in increased cartilage breakdown leading to decreased cartilage volume and joint space narrowing (12). OA is a debilitating, progressive joint disease often associated with aging process. It presents a combination of several disorders in which biomechanical properties of cartilage are altered leading to tissue softening and finally degradation. The degenerative process may be initiated with loss of proteoglycans from the ECM followed by disruption of collagenous fibrillar network leading to cell apoptosis/necrosis and deterioration of the functional tissue. Any synovial joint can develop osteoarthritis but knees, hips and small hand joints are the most common sites affected. Injurious and excessive mechanical stress can also result in reduction of proteoglycans and damage the collagen network. Mechanical factors leading to joint damage are factors that compromise 
joint protection and excessive loading. The first category comprises factors increasing joint vulnerability from deformation, muscle weakness, genetic predisposition and aging. The second implicates obesity, certain physical activities and trauma $(13,14)$. OA is a disorder affecting about $10 \%$ of the United State of America population older than 30, and most of the people over the age of 65 , with total costs estimated at $\$ 28.6$ billion/year (15).

\subsubsection{Articular cartilage repair conventional therapies}

One of the first therapies used to repair cartilage injuries was a conservative one; medications. Unfortunately it was rather than a cure a temporary relief of symptoms, which lead clinicians to surgical strategy (16). Today, pain caused by advanced arthritis in joints is palliated by total joint replacement by a synthetic prosthesis, therapy known as total hip or knee arthroplasty. Recently, and in order to avoid such a traumatic clinical treatment, therapies based on the stimulation of the natural repair process of cartilage have started to be practiced. In this sense, one of the first methods proposed to repair articular cartilage involved the disruption of subchondral bone to induce fibrin clot formation and initiate primitive stem cell migration from the bone marrow into the cartilage defect site. The fibrin clot formation is supposed to stimulate mesenchymal cells to initiate a repair response and differentiate into chondrocytes under the biological and biomechanical conditions experienced in the joint. These techniques, called bone marrow-based repair techniques, are techniques such as abrasion, subchondral drilling and microfracture. Abrasion arthroplasty is an abrasion of the damaged surface down to bleeding bone. The philosophy is that blood vessels will nourish the damaged area and primitive blood cells will reform into joint cartilage cells and 
cover the damaged area with a new healthy surface. It is usually kept for smaller areas, as it has been found that the material which grows, fibrocartilage, is not as strong as proper cartilage and may break down (17). Subchondral drilling is a surgical technique, no longer popular, where a high-speed drill is used to drill down through a defect in the joint surface and into the underlying bone, in order to allow the release of blood and bone marrow cells which have the potential to form a protective fibrocartilage layer over the defect. Because the high speed drill can cause heating of the bone, and consequent damage, the procedure has largely been replaced with less damaging techniques such as microfracture (18). Microfracture was designed to be less invasive than drilling. Multiple microfractures (3 to $4 \mathrm{~mm}$ apart) are created in the subchondral bone to disrupt blood vessels and allow fibrin clot formation (19). Although these therapies are able to produce a regenerated tissue, they lead to the formation of fibrocartilage in the cartilage defect. Fibrocartilage lacks the structural and mechanical properties of the original hyaline cartilage; it does not produce proper compressive stiffness against applied mechanical load and is excessively deformed under physiological loading. Then, the supposed reparative tissue formed recurrently degenerates and fissures (16).

More recent surgical strategies plan to replace the defect with patches or grafts. Cartilage allograft has problems with preparation and storage, and chondrocytes expire during the process. If implanted rapidly, cartilage allograft is supposed, by immune reaction from the synovial fluid, to induce tissue formation $(20,21)$. Autografting techniques, such as mosaicplasty with autologous osteochondral graft mimicking the defect dimension, lead to better results than other techniques. This technique is easily applied and the grafts are self-secure to the subchondral bone without any additional fixing procedure. However, simple cartilage grafts are difficult to fix as the graft requires a join to the native cartilage by 
sutures. This is a process that takes time and which may damage the native tissue. Although mosaicplastic grafts are more firmly implanted, they are limited to a few autogenous donor sources in the human body meaning the therapy is only valid for small cartilage defects and artificially constituted extra cartilage is needed to repair larger defects (22).

Solutions to the clinical problem of articular cartilage repair need multidisciplinary collaboration from biotechnology. In 1994 Brittberg and collaborators (23) introduced the technique of Autologous Chondrocyte Implantation (ACI). This technique consists of harvesting a minimum amount of autogenous cartilage to retrieve chondrocyte, which are cultured in vitro to expand the population, seeded onto the defect and covered with a flap $(13,24-26)$. Periosteum or perichondrium is used as a sutured flap to cover the transplantation of chondrocytes or progenitors cells in the defect site (PACI), but problems of abrasion force leads to suture failure and an unsuccessful outcome (27-30). However, the donor site morbidity produced by the use of a periosteal flap and reports of the emerging complication of graft hypertrophy encouraged the development of the second generation of ACI techniques. Collagen-covered ACI (CACI) replaces a periosteal flap with a porcine collagen membrane as a graft covering, avoiding donor site morbidity associated with harvesting periosteum and theoretically reducing the risk of graft hypertrophy (31). Successful clinical results with CACI suggest that a periosteal membrane is not essential for ACI to be successful (32). PACI and CACI methods both require the chondrocytes suspension to be injected under the chosen covering and cannot ensure even cell distribution within the final graft. In addition, both methods require the graft to be sutured in place, potentially requiring extended operating times where multiple grafts or concurrent procedures are required. In an effort to address these issues while continuing to avoid graft hypertrophy and donor-site morbidity, Matrix-Induced Autologous Chondrocyte 
Implantation (MACI) was developed. In MACI technique the cells are seeded onto a biodegradable porous matrix to constitute cartilage. The elements of MACI have been improved continually to regenerate cartilage of better quality (33). MACI is the first step to what is known as tissue engineering (TE) approaches. We will dedicate the following sections to specific topics associated to articular cartilage TE and bone TE, as the main objective of this doctoral thesis is the development of a synthetic construct for osteochondral tissue engineering to repair osteoarthritic joints.

\subsubsection{Tissue engineering (TE) approach for the regeneration of articular cartilage}

A commonly applied definition of tissue engineering, as stated by Langer and Vacanti in 1995, is "an interdisciplinary field that applies the principles of engineering and life sciences toward the development of biological substitutes that restore, maintain, or improve tissue function or a whole organ" (36). In 2006 Williams suggested that "tissue engineering is the creation of new tissue for the therapeutic reconstruction of the human body, by the deliberate and controlled stimulation of selected target cells, through a systematic combination of molecular and mechanical signals". Most of all adult tissues have limited ability to regenerate. In order to develop this ability it is necessary to give to the tissue some cues or signals. Being TE a matter of creation of new tissue, the process is logically expected to be achieved by cells. The cells need to be stimulated in order to create the new tissue by factors like biomolecules and support. The support structure may be the key for proper regeneration and can be a scaffold, a matrix, or a membrane within or on which the cells will express the new tissue. And they will be persuaded to do this by the molecular signals provided by relevant cytokines, growth factors or other 
molecules and mainly by mechanical signals transmitted through the support and the fluid medium $(34,35)$.

One of the main concerns in cartilage TE is cell sourcing. The optimal cell source for cartilage tissue engineering is still being identified. Chondrocytes, fibroblasts, stem cells and genetically modified cells have all been explored for their potential as a viable cell source for cartilage repair. Chondrocytes are the most obvious choice, since they are found in native cartilage and have been extensively studied to assess their role in producing, maintaining and remodelling the cartilage ECM. Fibroblasts are easily obtained in high numbers and can be directed toward a chondrogenic phenotype. Recent work has focused on stem cells, which have multi-lineage potential and can be isolated from a plethora of tissues. These progenitor cells can be expanded through several passages without loss of differentiation potential. Additionally, all of these cells can be modified genetically to induce or enhance chondrogenesis. The goal is to find an ideal cell source that can be easily isolated, is capable of expansion and can be cultured to express and synthesize cartilagespecific molecules (e.g., type II collagen and aggrecan).

In cartilage TE scaffolds have an important role acting as cell carrier and mechanical support. The primary focus has been on polymeric materials, in forms of hydrogels, sponges and fibrous meshes. Scaffolds provide a $3 \mathrm{D}$ environment that is desirable for the production of cartilaginous tissue. Ideally the scaffold should:

1) have directed and controlled degradation,

2) promote cell viability, differentiation, and ECM production,

3) allow for the diffusion of nutrients and waste products,

4) adhere and integrate with the surrounding native cartilage,

5) span and assume the size of the defect and

6) provide mechanical integrity depending on the defect location. 
Materials used to create these scaffolds can be natural polymers, synthetic artificial polymeric materials or combinations. Hyaluronic acid hydrogel is one of the most natural polymers used in this field. As it is naturally present in cartilage tissue it promotes cell proliferation, morphogenesis, inflammation and wound repair (37), and can easily fill defects and be crosslinked to increase its mechanical properties (38). Other natural hydrogels alternative to hyaluronic acid are collagen and fibrin and have been widely used for this aim (39-41).

Many different scaffolds have been constructed from other varieties of biomaterials. Biodegradable synthetic polymers such as poly(glycolic acid) (PGA), poly(lactic acid) (PLA) and their copolymer of poly(DL-lactic-co-glycolic acid) (PLGA) are good material candidates. These two kinds of biomaterials have their respective advantages and disadvantages. These synthetic polymers have relatively good mechanical strength and their shape and degradation rate can be easily modified, but their surfaces are hydrophobic and lack cell-recognition signals. Naturally derived polymers have good cell interaction and hydrophilicity but they are mechanically too weak to maintain the desired shape until newly formed tissue matures. A novel kind of hybrid porous scaffolds have been investigated by introducing porous collagen sponge into the pores of synthetic polymer sponge and demonstrated its ability for cartilage tissue engineering (42). Biodegradable polymers for cartilage regeneration need to improve the hydrophilicity to hinder cell attachment and protein adsorption on their surface. It is widely accepted that if chondrocytes adhere to the pore walls of a scaffold, despite the $3 \mathrm{D}$ environment, they tend to dedifferentiate and produce an ECM which is more abundant in collagen I than in collagen II (43). For an adequate regeneration cell arrangement should be more similar to what occurs in nature, where chondrocytes are situated in lacunae embedded into a hydrophilic ECM. Cell 
adhesion to scaffold pore walls is a phenomenon to be avoid. Several strategies have been reported to reduce cell-adhesive properties; plasma treatment and chemical graft of hydroxyl, carboxyl, or amide groups are examples of surface modifying in order to increase hydrophilicity $(44,45)$.

Not only the nature of the scaffolds has influence on the healing role of the implant, but also the percentage of porosity and the pores dimension. It has been studied that scaffolds with pores from 200 to $400 \mu \mathrm{m}$ and $90 \%$ porosity provide an optimum environment for chondrogenesis $(46,47)$. Surface roughness also affects cellular adhesion, proliferation and phenotype (48).

Stimulating factors, such as growth factors, are another component of TE triad. Growth factors act on target cells by adhering to a specific receptor which triggers, by a system of signals or messengers, the activation of nuclear genes that determine cell proliferation, differentiation and death. Growth factors combine to promote cell survival and induce chondrocytic proliferation. A number of growth factors like TGF- $\beta$, FGF, BMP and IGF, along with other soluble factors like HA, chondroitin sulfate, and insulin, have been explored for their effects on cartilage tissue engineering (49).

There is significant evidence that dynamic compressive loading has a stimulatory effect on cartilage, chondrocytes, and stem cells. Chondrocytes exhibit compressibility and behavioral changes with compressive load as a function of strain. Numerous loading regimes ranging from a single application to continuous loading have been investigated as a means to accelerate and improve tissue formation $(50,51)$.

Bioreactors have been employed to accelerate and improve the growth of engineered cartilage in vitro. They serve to enhance nutrient transport and provide a hydrodynamic environment that imposes a fluid-induced shear stress to promote the synthesis of cartilage-specific matrix proteins. Dynamic cell 
seeding of porous scaffolds has led to faster invasion and better cell distributions (52-54).

\subsubsection{TE approach for the regeneration of bone}

Bone and cartilage cells come from the same embryonic stem cells, and while studies show how bone stem cells are involved in cartilage repair it is first necessary to study the status of bone TE independently. The remodelling process of bone gives to bone a high ability to regenerate naturally. But some defects, because of the dimension or severity, cannot be cured naturally. In this context TE is an alternative to the limit of bone regeneration.

Natural bone grafts represent one of the techniques for bone repair, but there are some important drawbacks. Harvesting the graft requires additional invasive surgical procedures that may lead to donor morbidity and infections. The use of autografts is not highly available, unlike autologous bone, allogeneic (from human cadaver) and xenogeneic (from animal source) grafts which are widely available and do not require additional surgery on the patient. However, allogeneic bone has to undergo processing techniques such as lyophilization, irradiation or freeze-drying to remove all immunogenic proteins in order to avoid any risk of immunogenic reaction. These processing techniques have a negative effect on the potential of the allografts, which consequently decrease their biological performance when compared to autografts (55).

Because of the above mentioned drawbacks of natural bone grafts, a large number of synthetic grafts have been developed. As for cartilage tissue engineering, bone tissue engineering presents the same areas of focus, such as cell sourcing, scaffolds design and stimulating agents.

One of the most common cell sources for bone tissue engineering is bone marrow stromal cells (bone marrowderived mesenchymal stromal cells) (56). Endothelial cells are 
one other source of cells to seed scaffolds for bone repair. They can be isolated from the umbilical cord or from human dermis, for example (57). It has been studied that human primary fetal bone cells could be of great interest for bone research, due to their fast growth rate and their ability to differentiate into mature osteoblasts (58). Another source of cells can be adipose stem cells, called lipoaspirate cells as they share the characteristics of human osteoprogenitor cell surface markers similar to bone marrow mesenchymal stem cells $(59,60)$.

Scaffolds provide support in the formation of tissue, playing a crucial role in cell growth and differentiation. These goals are achieved by an appropriate chemistry, morphology and structure of the scaffold to accelerate cell adhesion for osteoblasts and osteoprogenitor cells, migration, differentiation and synthesis of the homogenous bone matrix avoiding the necrosis in the central regions. Various combinations of matrix materials are well studied, including biodegradable synthetic polymers such as poly(glycolic acid) (PGA), poly(lactic acid) (PLA) (61), copolymers of poly(DLlactic-glycolic acid) (PLGA) (62) and poly( $\varepsilon$-caprolactone) (PCL) (63), and biodegradable naturally derived polymers such as collagen (64), fibrin (65) or silk $(66,67)$.

High porosity and large pores enhance bone ingrowth and osseointegration of the implant after surgery, as such, the higher pore surface area, interacting with the host tissue can accelerate degradation due to macrophages via oxidation and/or hydrolysis. In vitro lower porosity enhances osteogenesis due to cell aggregation and suppressed proliferation. The recommended pore size for a scaffold is 200$500 \mu \mathrm{m}$. Relatively larger pores favor direct osteogenesis, since they allow vascularization and high oxygenation, while smaller pores result in osteochondral ossification $(68,69)$. Thus big pores should be interconnected in order to allow the flux of cells and the supply of oxygen and nutrients to cells inside the scaffolds and facilitate waste out of the scaffold and 
vascularization (70). There is, however, an upper limit in porosity and pore size set by constraints associated with mechanical properties. An increase in the void volume results in a reduction in mechanical strength of the scaffold, which can be critical for regeneration in load-bearing bones (68).

One of the important needs for scaffolds is to support load. However the specific mechanical requirements of the scaffolds from the beginning of implantation till complete healing is still under debate. For example, some authors have stated that scaffold mechanical properties should match those of natural bone. In the case of cortical bone the elastic modulus is from 12 to $18 \mathrm{GPa}$ and 1 to $5 \mathrm{GPa}$ for trabecular bone. But some authors state simply that there should be enough if the scaffold does not collapse during handling and during the patient's normal activities (71). Most of the porous scaffolds listed reveal insufficient mechanical properties (Figure 1-4). Figure 1-4 shows that some dense polymers match cancellous bone properties and approach cortical bones as well. Porous scaffolds however are at least one order of magnitude weaker than cancellous bone and orders of magnitude than cortical bone. Bioactive ceramics have less stiffness than the biodegradable scaffolds and the porous composites present a slight increase of mechanical properties compared to porous polymer scaffolds (72). A wide range of bioactive inorganic materials similar in composition to mineral phase of bone are used in the fabrication of composite scaffolds for bone regeneration, e.g. tricalcium phosphate, hyadroxyapatite, bioactive glasses and their combination (73). The composite is an alternative to generate bioactive materials which combine the bone nature of an inorganic phase with the toughness of a polymer phase (74). 


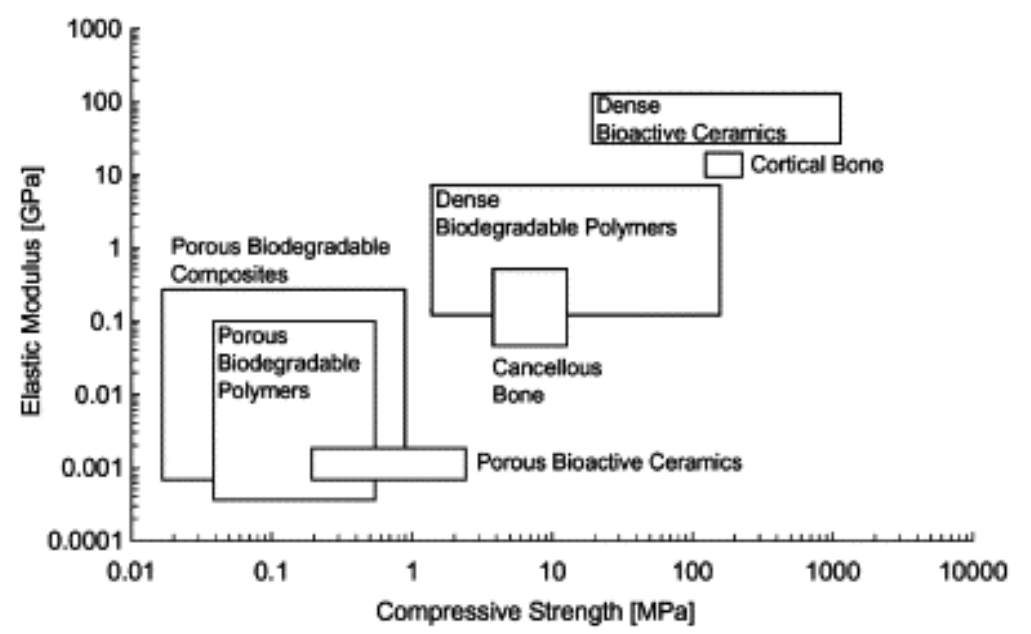

Figure 1-4: Elastic modulus vs. compressive strength of biodegradable polymers, bioactive ceramics, composites, cancellous bone and cortical bone. Porosities of the porous scaffolds are superior of $\mathbf{7 5 \%}$ and mostly interconnected. Reproduced from (72).

Cells seeded on tissue engineering constructs are often loaded by directly adding a cell suspension to the scaffold. Although this method is simple and thus widely used, it can result in low seeding efficiencies and non-homogenous seeding distributions. Seeding efficiency can be improved by placing porous scaffolds in a mixing cell solution. Spinner flask systems have been used for this purpose. Bone marrow stromal cell and chondrocyte loading efficiency have been shown to be $20 \%$ higher using a perfusion system as compared to static and spinner flask loading and significantly more uniformly distributed. Dynamic seeding yielded higher seeding efficiencies under most culture conditions. Perfusion seeding may increase seeding efficiency and reduce risk of contamination by decreasing the amount of time the scaffolds are handled outside the bioreactor system (75).

Bone contains a cocktail of growth factors including transforming growth factor beta (TGF- $\beta$ ), platelet-derived growth factor (PDGF), bone morphogenetic proteins (BMPs), 
insulin-like growth factors I and II (IGF-I and IGF-II) and fibroblast growth factors (FGFs) that have been shown to have important actions in bone cell function and therefore have been used to induce adequate bone cell response and enhance bone formation. Bone seems to be the largest reservoir for transforming growth factor- $\beta$ (TGF- $\beta$ ). TGF- $\beta$ is a secreted multifunctional protein that regulates many aspects of cellular function, including cell proliferation, differentiation and extracellular matrix metabolism. Insulin-like growth factors (IGFs) are also considered to be among the most important skeletal growth factors. The response to BMPs differs from the response to TGF- $\beta$ in that BMPs (BMPs 2, 4 and 7) induce bone development from connective tissue perivascular cells (or other osteoprogenitor cells), which in the lifetime of the animal would not normally develop bone, i.e. they are osteoinductive $(76,77)$. The significance of enhanced osteogenic effect with multiple growth factors has been well established. Enhanced bone formation was observed with application of TGF- $\beta 1$ and IGF-1 simultaneously. Similarly, TGF- $\beta 3$ and BMP-2s loaded alginate scaffolds had significantly enhanced bone formation in mice in comparison to single growth factor loaded scaffolds. It has been suggested that multiple growth factor use can also be cost effective by yielding better results with reduced amounts of growth factor utilization when compared to much higher doses of single growth factors required for comparable results (78).

Cells regularly contract and thereby deform the scaffolds, resulting in the generation of tension within the cells. This contractile activity of cells is essential for mechanotransduction, which in vitro is provided by the bioreactor. As a part of mechanotransduction, cells generate and experience forces which affect the kinetic rate of proteinprotein interaction. MSCs when subjected to the mechanical stimulations accelerate the expression of osteogenic markers on the cell surface. Bioreactors offer a controlled environment 
to cells. The fate of cell proliferation and differentiation according to environmental stress can be induced by fluid flow and/or mechanical stimuli such as dynamic, static or shear forces, and other physical cues such as oxygen tension and $\mathrm{pH}$ effects. Several bioreactor designs have been evaluated for in vitro bone-tissue engineering applications: rotating wall-vessel bioreactor, hollow fiber bioreactor (HFBR), electromagnetic bioreactor, compression-perfusion bioreactor and perfusioncompression bioreactor. The HFBR system allows high cell densities, preferential for the functional development of bone cells, while in an electromagnetic bioreactor, electromagnetic wave stimulation enhances cell proliferation and growth factor secretion. A significant extra cellular matrix synthesis is claimed when osteoblasts like cells are treated with an electromagnetic wave. The electromagnetic stimulation increased the net $\mathrm{Ca}^{2+}$ flux in human osteoblast like cells. Magnetic up-regulation of type-I collagen has been introduced representing the most abundant and significant structural protein of the bone matrix. On the other hand, compressionperfusion stimulation enhances the metabolic activity of boneforming cells, including ECM synthesis (79).

\subsection{OSTEOCHONDRAL TISSUE ENGINEERING}

\subsubsection{Purpose of the strategy}

Osteochondral TE can be used in the case of cartilage defects to penetrate the subchondral bone. In cases where the lesions do not penetrate the subchondral bone, the pluripotent progenitor cells from the bone marrow cannot be recruited and the repair will be limited. It would be desirable to use biphasic constructs, which could satisfy the mechanical and biological requirements of both tissues. Even in chondral defects, the use of single scaffolds intended to regenerate cartilage may not be effective, as it is difficult to integrate the implant with the surrounding tissue. In that situation strategy could consist in 
creating a full thickness defect and a posterior implantation of an osteochondral implant, this will help to anchor the graft into the defect since, as commented, bone-to-bone interface integrates better and faster than cartilage-to-cartilage interface (80). One of the main parameter to take care of is the interface between bone and cartilage. Indeed cartilage is a soft tissue compared to bone which is a hard one, so the connection is characterized as a soft-to-hard tissue interface. Cartilage-tobone interface are critical for joint motion and stabilization. It is characterized by gradual changes in properties and structural organization from one tissue to another, as from cartilage to bone mineralization is gradually detected. Interfaces of tissues are complex in structure, and they have a relatively small length scale; around $1 \mathrm{~mm}$. For all these reasons and in matter of nature imitation osteochondral TE is widely studied in the case of cartilage defects $(15,81,82)$.

\subsubsection{Layered scaffolds for osteochondral TE: an overview}

The ultimate researches in cartilage TE lead to the osteonchondral repair as an alternative to cartilage repair strategy. One approach to deal with articular cartilage repair from subchondral bone could be from single-layer scaffolds. That would be the concept of cartilage tissue engineering mentioned previously. An alternative strategy could include the construction of bilayered structures (Figure 1-5), especially adequate to incorporate, in a favourable environment two types of cells requiring different chemical surroundings, mechanical features and pore morphology. This would allow the growth of two different tissues, satisfying the different biological requirements. Therefore, heterogeneous structures should be built, in which one of the sides promotes cartilage regeneration and the other region, exhibiting different properties, is designed to encourage bone integration (15). In 
the case of bilayered scaffolds the concept of osteochondral engineering is the fusion of cartilage and bone tissue engineering, which were discussed above. One important parameter in the bilayered scaffold is the interface between the two parts (81-83).

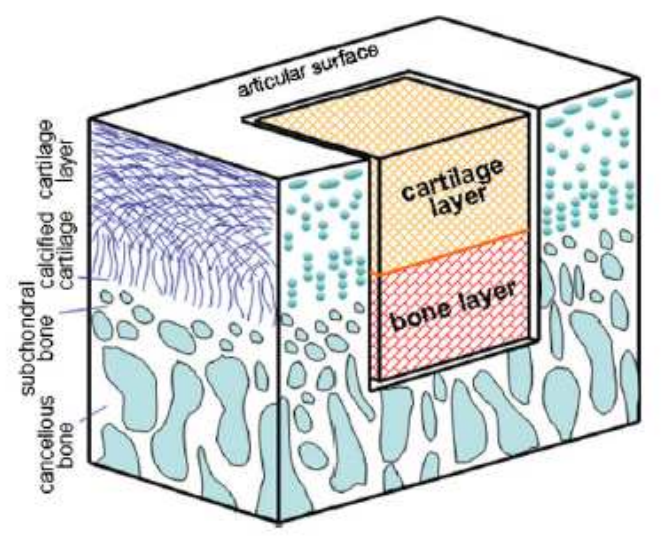

Figure 1-5: Schematic representation of osteochondral defect, of the cartilage layer (upper side) and the bone region (bottom) and the osteochondral implant. Reproduced from (15).

Different techniques are used to produce osteochondral scaffolds and to induce tissue regeneration. The easiest way to construct a biphasic scaffold is to build two layers independently and join them prior implantation. There are some bilayered scaffolds commercialised such as "Chondromimetic"TM" (Orthomimetics / Tigenix Ltd., Cambridge UK) (84) made from collagen and-GAG for the chondral part and collage-GAG and calcium phosphate for the bone part, which present satisfactory results after 26 weeks in osteochondral defects of goats. They support the repair of osteochondral defects, and a maturation of regenerated tissue towards hyaline structure. The production of gradient biomaterials to engineer grafts for full reconstruction of softto-hard interface tissues is one promising strategy (84). Bi et 
al. made biphasic scaffolds made from collagen-chitosan mixtures for the chondral phase and a composite of bioactive glass and collagen for the osseous phase. Collagen integrated in the two respective phases connected by cross linking (85). Chen et al. fabricated biphasic scaffold made of collagen sponge and a composite of PLGA with collagen for the cartilage and bone part respectively. Collagen sponge in the two respective layers was connected. Osteochondral tissue was regenerated four months after implantation in the knee of a one-year beagle. Previously canine bone-marrow-derived mesenchymal stem cells were cultivated in the biphasic scaffold (86). Ghosh et al, fabricated bi-layerded construct based on poly(L-lactic acid) and starch for osteocondral defect. In both side PLLA was used. A blend of starch and PLLA was used in the cartilage side, which was found to possess adequate hydratation capability. For the bone region, PLLA reinforced with hydroxyapatite was used. The bone like layer exhibited a bioactive like behaviour within 30 days in SBF. The two layers were not separated by a compact zone (83). Cui et al. studied autologous chondrocytes and osteoblasts seeded on scaffolds of PLGA for the cartilage part and tricalcium phosphate (TCP) for the bone part to generate a biphasic scaffold sutured by absorbable suture. The scaffolds were implanted in femoralmedi condyles of mini-pigs during six months. The cartilage regenerated was similar to host cartilage, subchondral bone was partly replaced by neocartilage (87). As it was discussed previously, in order to mimic human nature it is necessary to reproduce a gradient cartilage-to-bone structure when oesteochondral layered scaffolds are designed. Kon et al. designed three layered scaffolds from cartilage to bone made of a first layer of collagen I (100\%), a transitional one of hydroxyapatite (HAp) (40\%) and collagen I (60\%) and a third one of HAp (70\%) and collagen I (30\%). The scaffolds were implanted during 6 months in femoral condyles, patellas and throchleas in 
humans as a pilot clinical study. This study showed to be promising, scaffolds maturation in bone and cartilage tissues showed normal osteochondral structure in $60 \%$ and a not differentiated inhomogeneous signal in the implanted area in the rest of the patient (88). Further, in the mimetic approach Lien et al. designed a four layer scaffold made of: a porous ceramic layer as osseous component and also as anchor, a dense ceramic layer to prevent blood vessel penetration, a porous ceramic layer for the fixation of bone to cartilage layer (i.e, joining the ceramic part to a porous gelatine solution into the porous ceramic layer) and a latter layer of gelatine as cartilaginous component. The joining was done by infiltration of gelatin solution into the porous ceramic layer, gelling and crosslinking. The scaffold was tested by in vitro cell culture with rat's joint chondrocytes for 4 weeks, after that time cartilage tissue could be developped (81).

\subsection{BIOACTIVITY}

\subsubsection{In vivo bioactivity}

Materials for bone tissue engineering need to be osteoconductive and so able to support bone growth and encourage the ingrowth of surrounding bone and also should be capable of osteointegration, which means able to integrate into the surrounding bone. Biocompatibility ensures that a biomaterial does not produce a foreign body reaction (continuous presence of macrophages and giant cells in its sorroundings) and can be used without any damage to surrounding tissues and organs as well as to the whole organism. Surface reactions in osteoinductive materials stimulate both intracellular and extracellular responses at the interface of the material with its physiological environment, giving rise to rapid in vivo bone regeneration. They are, thus, osteoproductive materials. Slower surface reactions and only 
extracellular responses occurring at the interface, lead to bone migration along the interface of the so called osteoconductive materials. These materials allow only bone ingrowth, therefore showing osteoconductivity $(55,89,90)$.

The bone-bonding biomaterials, so-called bioactive or surface active, are biocompatible materials that form a layer of hydroxyapatite similar to the apatite in bone on their surface when implanted in the body, and bond to the living bone through this apatite layer $(87,91)$. In vivo bioactivity of a material can be assessed in vitro. In 1991, Kokuko proposed an in vitro test to assess the bioactivity of a material by its capacity to generate a layer of hydroxyapatite (HAp) on its surface when immersed in simulated body fluid (SBF) (92). $\mathrm{SBF}$ is an aqueous medium containing all the salts present in the human plasma at physiological temperature $\left(37^{\circ} \mathrm{C}\right)$ and $\mathrm{pH}$ (7.4) (93). A comparison of the concentrations of ions ( $\mathrm{mmol} / \mathrm{l})$ in human plasma and in SBF is given in Table 1-1. SBF simulates only the inorganic part of human blood plasma and does not contain proteins, glucose, vitamins, hormones, cells, $\mathrm{CO}_{2}$ flow, etc., which are factors influencing the solubility of salts, or which interact in the mineralization process of bone in vivo. It is interesting to know that the composition of blood plasma is not fixed to an amount of ions (i.e. $\mathrm{K}^{+}, \mathrm{Ca}^{2+}$, $\mathrm{Cl}$ - and $\mathrm{HPO}_{4}{ }^{2-}$ ) and can vary depending on the person's sex, age and nutrition $(94,95)$. This test is a first step to determine the intrinsic ability of the material to form crystals of hydroxyapatite on its surface. The main quality of this test is that it does not require expensive equipment to give such information as the in vivo "mineralisation" ability of a material. Many researchers are using this in vitro test to predict the ability of the material to be in vivo bioactive (96, 97). Kokubo et. al studied the ability of materials such as ceramics and metals to nucleate hydroxyapatite of its surface $(98,99)$. Olmo et al. studied the biocompatibility of HAp coated on the surface of bioactive ceramics after immersion in SBF 
during 7 days. It was proved that high cell attachment and good biocompatibility of the samples covered with biomimetic HAp (100). Zhang et al. could nucleate a layer of biomimetic HAp on the surface of poly(L-lactic acid) (PLLA) porous scaffolds after 2 weeks of immersion in SBF (101). Wu et al. were able to observe nuclei of biomimetic HAp on the surface of apatite ceramic scaffolds after 3 days in SBF (102). Taddei et al. could nucleate a layer of biomimetic HAp inside and outside PCL scaffolds after 28 days in SBF (103), and Leeuwenburgh et al. concluded a good in vitro mineralization of Ti discs after 12 weeks in SBF (104). Our research group in the Centre for Biomaterials and Tissue Engineering has also tested the nucleation ability of HAp by immersion in SBF different types of scaffolds. For example, Myriam Lebourg made similar studies on composite scaffolds of PCL and hydroxyapatite (105). Vallés et al, studied the ability of $\mathrm{P}$ (EMA-co-HEA)/ $/ \mathrm{SiO}_{2}$ hybrid nanocomposites to nucleate hydroxyapatite on its surface and the influence of surface modifications on the bioactivity $(106,107)$.

Table 1-1: Ion concentration in simulated body fluid and human blood plasma.

\begin{tabular}{ccccccccc}
\cline { 2 - 8 } & \multicolumn{7}{l}{ Ion molarity $(\mathbf{m M})$} \\
\cline { 2 - 8 } & $\mathrm{Na}^{+}$ & $\mathbf{K}^{+}$ & $\mathbf{M g}^{2+}$ & $\mathbf{C a}^{2+}$ & $\mathbf{C l}^{-}$ & $\mathbf{H C O}_{3}{ }^{-}$ & $\mathbf{H P O}_{4}{ }^{2-}$ & $\mathbf{S O}_{4}{ }^{2-}$ \\
\hline $\begin{array}{c}\text { Blood } \\
\text { Plasma }\end{array}$ & 142.0 & 5.0 & 1.5 & 2.5 & 103.0 & 27.0 & 1.0 & 0.5 \\
\hline SBF & 142.0 & 6.5 & 1.5 & 2.5 & 148.0 & 4.2 & 1.0 & 0 \\
\hline
\end{tabular}




\subsubsection{Bioactive materials and mechanism of apatite formation}

Precipitation of Ca-P phases in aqueous solutions mainly include dicalcium phosphate $\left[\mathrm{CaHPO}_{4} \cdot 2 \mathrm{H}_{2} \mathrm{O}\right.$, DCP], octacalcium phosphate $\left[\mathrm{Ca}_{8}\left(\mathrm{HPO}_{4}\right)_{2}\left(\mathrm{PO}_{4}\right)_{4} \cdot 5 \mathrm{H}_{2} \mathrm{O}, \mathrm{OCP}\right]$ and hydroxyapatite $\left[\mathrm{Ca}_{10}(\mathrm{OH})_{2}\left(\mathrm{PO}_{4}\right)_{6}, \mathrm{HAp}\right]$. HAp is considered the most thermodynamically stable in a physiological environment. OCP and DCPD, however, have been regarded as precursors of HAp or the metastable phases of Ca-P because they are kinetically favourable. $\mathrm{pH}$ is a parameter influencing the nature of HAp nucleated, as only in physiological conditions, $\mathrm{pH}=7.4$ at $37^{\circ} \mathrm{C}, \mathrm{HAp}$ is favourable to precipitate (108). Equation (1) presents the ion units of HAp for precipitation in aqueous solutions.

$$
5 \mathrm{Ca}^{2+}+3 \mathrm{PO}_{4}^{3-}+\mathrm{OH}^{-} \rightarrow \mathrm{Ca}_{5}\left(\mathrm{PO}_{4}\right)_{3}(\mathrm{OH})
$$

In bone TE many materials are studied and revealed to be bioactive i.e. able to nucleate a layer of biomimetic hydroxyapatite on their surface. Most studies deal with the use of biodegradable polymers filled with bioactive particles and ceramics to enhance bioactive potential of biomaterials for bone TE. Poly( $\varepsilon$-carprolactone) (PCL) filled with nanoparticles of HAp materials are able to nucleate a layer of biomimetic HAp on their surface after two weeks in SBF, the layer observed presented cauliflower shape characteristic of HAp and physical characteristics similar to biological HAp (103,109-110). Similar conclusions have been made in the case of poly(L-lactide acid) containing bioactive glass ceramic (111) and also for poly(D-L-lactide and poly(lactide-co-glycolide) containing bioactive glass (112). Bioactive glasses and calcium phosphates present a good bioactivity due to their close chemical and crystal resemblance to bone mineral. Being more 
brittle than polymers they are usually used to reinforce polymeric scaffolds and activate their bioactivity $(113,114)$.

The formation of a precursor of hydroxyapatite is shown in Figure 1-6. The surface of the polymer is negatively charged when immersed in SBF due to the presence of carboxylic and hydroxyl groups (115). Consequently the negative charge attracts the positive ions of the aqueous medium. First an amorphous calcium phosphate layer is formed. Once the amorphous precursors of apatite or apatite nuclei are formed, they can grow spontaneously by consuming the calcium and phosphate ions from the surrounding body fluid, because the body fluid is supersaturated with respect to the apatite (116). During this process, the amorphous calcium phosphate incorporates $\mathrm{OH}^{-} \mathrm{CO}_{3}{ }^{2-}, \mathrm{Na}^{+}, \mathrm{K}^{+}$and $\mathrm{Mg}^{2+}$ ions from the solution and finally crystallizes into HAp, which is the most stable calcium phosphate in aqueous media, rendering a HAp layer of polycrystalline agglomerates of carbonated hydroxyapatite on the substrate (72).

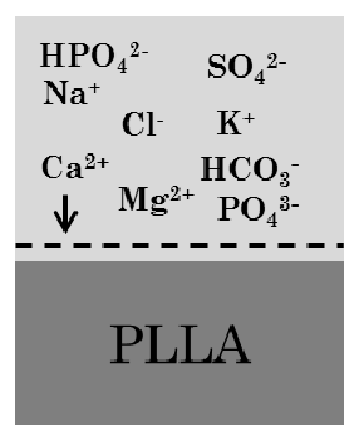

Formation of negative layer on the surface of PLLA

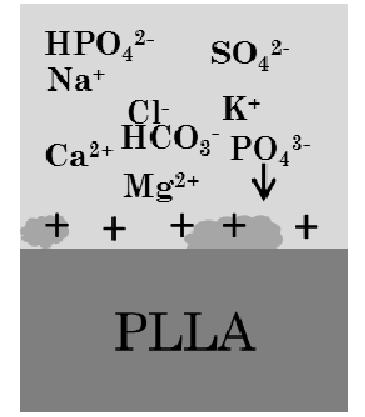

$$
\begin{gathered}
\text { Formation of } \\
\text { amorphous } \\
\text { caleium } \\
\text { phosphate layer } \\
\text { on PLLA surface }
\end{gathered}
$$

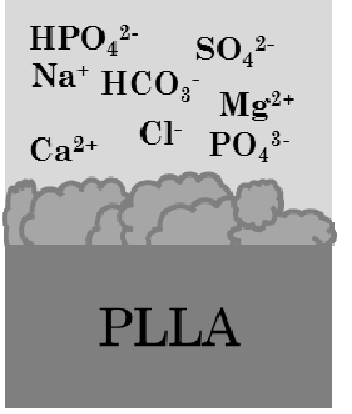

Formation of crystalline apatite layer on PLLA surface

Figure 1-6: Mechanism of apatite nucleation on PLLA surface. Inspirated from (116). 
A variety of procedures have been proposed with the purpose of improving the bone-bonding ability of bioinert substrates or shortening the induction period required for apatite deposition from SBF. One of these procedures is the induction of the ability to nucleate bonelike apatite with the inclusion of calcium phosphate or glass ceramic nanoparticles in the polymeric matrix of the bioinert scaffolds. The mechanism proposed for this behaviour is that bioactive materials show negative surface potential when immersed in $\mathrm{SBF}$, due to deprotonation at physiological $\mathrm{pH}$ of groups like - $\mathrm{COOH}$, $\mathrm{OH},-\mathrm{TiOH},-\mathrm{SiOH}(106,107)$. This negative charge attracts calcium ions, then creating a Ca-rich layer with positive surface potential, which then attracts negative ions in solution like phosphate leading to the deposition of a Ca-poor layer. This process repeats itself generating the HAp layer on the surface of materials $(116,117)$.

Alternatively, prior to the immersion of the materials in SBF a technique has been developed consisting in the pre-nucleation of calcium phosphate elements on the surface of the material. This process is usually done by soaking the materials in aqueous $\mathrm{CaCl}_{2}$ and $\mathrm{K}_{2} \mathrm{HPO}_{4} 3 \mathrm{H}_{2} \mathrm{O}$ saturated solutions alternately three times. The soaking permits the deposit nuclei of calcium and phosphate on the surface of the materials and so creates local precursors to apatite formation (118).

Another procedure consists in the activation of the surface of the material itself in order to increase its intrinsic ability to form HAp on its surface. One technique currently used to activate the surface properties of polymeric materials is the treatment with aqueous $\mathrm{NaOH}$ solutions to introduce carboxylate groups onto the surface of the materials. The results of this immersion in the case of polyesters as PLLA are not satisfactory, as $\mathrm{NaOH}$ ions generate a rapid degradation of the material making it useless for its later implantation in bone. Plasma pre-treatment of the materials surface is an 
alternative to surpass these drawbacks. Plasma surface treatments from functional groups such as hydroxyl and carboxyl groups that increase the hydrophilicity of the materials and so enhance their osteogenenic ability (119-121).

\subsection{SYNTHETIC POLYMERS BIODEGRADATION}

We will refer in this section mainly to the poly(L-lactic acid) (PLLA) polyester since it was the selected material for the design of our osteochondral construct. Similar synthetic materials share some concepts that will appear in the section.

\subsubsection{Mechanisms of biodegradation}

The purpose of porous scaffolds is to degrade in the human body when the targeted tissue regenerates, and that its byproducts can be assimilated or excreted by a living system.

Synthetic polymers with ester functional groups as PLLA can degrade through hydrolytic way; enzymes such as pronase, proteinase $\mathrm{K}$ and bromelain accelerate the degradation process during the later stages of the in vivo degradation $(122,123)$.

The ester bond is a hydrolysable linkage leading to carboxyl endgroup and a hydroxyl one (eq. 2). The carboxyl endgroups formed are capable of catalysing the hydrolysis of other ester bonds, a phenomenon called autocatalysis.

$$
\mathrm{R} \sim \mathrm{COO} \sim \mathrm{R}^{\prime}+\mathrm{H}_{2} \mathrm{O} \stackrel{\sim \text { соон }}{\longrightarrow} \quad \mathrm{R} \sim \mathrm{COOH}+\mathrm{HO} \sim \mathrm{R}^{\prime}
$$

Three types of erosion processes are related to the degradation of biodegradable synthetic polymers: surface, bulk and autocatalysis. Mainly surface erosion and bulk erosion compete, in which bulk erosion can be with or without autocatalysis (124). In surface erosion the hydrolysis process occurs in the region near the surface leading to the erosion of 
the surface moving to the core of the material. At the surface of the scaffolds no autocatalysis can occur because being in aqueous the medium is always buffered. As in vitro and in vivo the carboxyl endgroups are in presence at the surface of the scaffolds, they are neutralised by the medium and consequently lose their catalytic potency. For this to be possible, carboxyl endgroups release has to happen at the same rate as neutralisation, which in some circumstances is not possible and in vivo degradation is faster than expected (124). Bulk erosion involves instantaneous water diffusion at the beginning uniformly in the polymer core, it is then the typical mechanisms found in hydrophilic materials. Water diffusion is normally very slow in the surface erosion process and normally it is typical of hydrophobic materials. Bulk erosion is a mechanism where all the chains or the polymer are degraded at the same rate and molecular weight decrease and reduction of the mechanical properties and mass loss of the material is produced at almost the same time in the whole material (124). In the case of autocatalysis soluble oligomeric species are generated, those which are close to the surface can escape from the matrix before total degradation, while those located inside can hardly diffuse out of the matrix. Therefore, autocatalysis is more characteristic of bulk erosion, but cannot be excluded in the case of surface erosion (124).

Enzymatic erosion is normally considered as a surface erosion mechanism, since enzymes are large molecules difficult to diffuse into the chains of the polymer (124).

The degradation process depends on various parameters, such as matrix morphology, chemical composition, molecular weight, size, distribution of chemically reactive compounds and the nature of the degradation media $(125,126)$. Once placed in an aqueous medium, water penetrates into the scaffold leading to hydrolytic cleavage of ester bonds. PLLA being hydrophobic its degradation is expected to be in surface. PLLA degrades by non-specific hydrolytic scission of its ester 
bonds, and yields lactide acid, which is a normal by-product of anaerobic metabolism in the human body; it can also degrade by enzymatic reaction (127). Complete degradation of one material, being the degradation rate dependent on the medium and the characteristics of the material, can take place in months or even years.

\subsubsection{Effect of different parameters on the rate of biodegradation}

For aliphatic polyesters, hydrolysis rates are affected by temperature, molecular structure, ester group density, type of degradation media and mechanical stimulus. Crystallinity is also an important parameter as the crystalline domains are less permeable to water penetration, slowing down hydrolysis. First, hydrolysis tends to affect to the amorphous regions of semicrystalline polymers with random hydrolytic scission of ester bonds. Then, hydrolysis starts to attack from the edge towards the center of the crystalline domains, when most of the amorphous regions are degraded. The molecular weight plays also a very important role in the degradation rate on polyesters; the higher the initial molecular weight, the slower the degradation rate as more ester bonds have to be attacked and diffusion of water is slower if polymer chains are longer $(128,129)$. The $\mathrm{pH}$ of the aqueous medium also affects the degradation rate, degradation in alkaline solution occurs faster as oligomeric acids are dissociated into $\mathrm{RCOO}$ presenting higher hydrophilicity which enables them to diffuse out in the solution, in contrast to non-dissociated oligomers $\mathrm{RCOOH}$ formed by hydrolysis in neutral or acid solution (130, 131). The dimension and the porosity of the scaffold influence the degradation process. The smaller a scaffold is the less is the difference between surface and centre due to autocatalysis (132). An increase of temperature can also increase diffusion, due to the flexibility gained by the polymer chains. 
1.5 POLY(L-LACTIC ACID) AND POLY(L-LACTIC ACID)/ INORGANIC COMPOSITE SCAFFOLDS

\subsubsection{Main properties of poly(L-lactic acid)}

As said before, in this work poly(L-lactic acid) (PLLA) has been selected in order to design porous scaffolds. Poly(L-lactic acid) is generated from lactic acid (2-hydroxypropanoic acid), which is an organic acid found in many products of natural origin. Lactic acid is produced by animals, in plants and by microorganisms in the nature. It can also be derived from intermediates with an origin in renewable materials or from chemicals derived from coal or oil. PLLA is one of the few polymers approved by FDA for intern biomedical applications. Lactic acid is composed by an asymmetric carbon atom, leading to two optically active configurations. It is one of the smallest optically active molecules, which can be either $\mathrm{L}(+)$ or $\mathrm{D}(-)$ stereoisomer (Figure 1-7). The $\mathrm{L}(+)$ isomer is the one naturally produced in humans and other mammals, but both are produced in bacterial systems $(133,134)$.

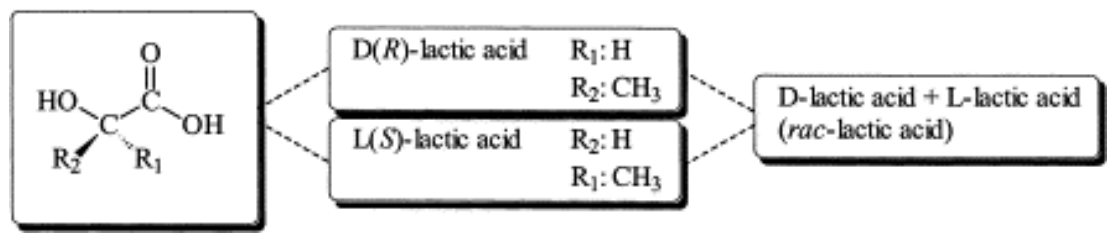

Figure 1-7: Stereoforms of lactic acid.

From the isomers L and D of PLA is it possible to obtain the poly(L-lactic acid) (PLLA) (Figure 1-8), poly(D-lactic acid) (PDLA) and poly(D, L-lactic acid) (PDLLA). 
<smiles>CCOC(=O)C(C)OC(C)C(=O)OC(C)C(=O)O</smiles>

Figure 1-8: Poly(L-lactic acid) formulation.

PLLA is synthesized by ring-opening polymerization of Llactide. The ring-opening polymerization route includes polycondensation of lactic acid followed by a depolymerisation into the dehydrated cyclic dimer, lactide (3,6-dimethyl-1,4dioxane-2,5-dione), which can ring-opening polymerized into high molar mass polymers (135).

PLLA is a semi-crystalline polymer, its thermal transitions, such as glass transition, melting and crystallization, are important aspects to know, since they determine the final properties of the PLLA chosen to fabricate the material for implantation. In general, it is difficult to obtain a PLLA with a crystallinity higher than $60 \%$. The crystallisation process of PLLA can be observed in the range of $80-160^{\circ} \mathrm{C}$ (136). PLLA exhibits a high melting temperature, around $173-178^{\circ} \mathrm{C}(137)$. The glass transition temperature of PLLA is about $55-65^{\circ} \mathrm{C}$, which is not so far above room or body temperature. That would explain the metastable state of the amorphous fraction at physiological temperatures, and slow changes in molecular configuration. The process of physical aging, also called structural relaxation, is a reversible physical process, which is not supposed to generate any chemical changes $(138,139)$.

PLLA is a polymer widely known to be biocompatible and biodegradable into non-toxic components which can be eliminated from the body in the form of carbon dioxide and water. The use of PLLA is also seen in biomedical applications such as scaffolds for tissue engineering or medical devices such as screws or sutures (140-143), as well as being a material used in industry for packaging or textile (135). 


\subsubsection{An overview to poly(L-lactic acid) scaffold fabrication techniques}

There are many techniques to fabricate porous scaffolds from poly(L-lactic acid). The main techniques used are basically solid free-form fabrication (SFF) techniques, electrospinning, moulding, freeze drying and freeze extraction. Each method leads to specific properties which characterise the final product, such as morphology, porosity, mechanical strength and so on. Other parameters to pay attention to at the time of choosing the technique of fabrication are the time it takes, its cost, complexity and repeatability.

SFF techniques require a computer model of the desired scaffold architecture from computer-assisted design. An inkjet printing system is directed by the computer-assisted design. A thin layer of polymer powder is spread over a pistons surface, the inkjet dispenses a binding liquid, which is a solvent for the polymer, in the desired pattern of the scaffold layer. After a short time, the piston is lowered by the thickness of a single layer and the subsequent layers of powder and binding liquid are applied. Unbound polymer remains in the network during the fabrication process to support disconnected sections in the layer. SFF techniques present the advantages of being rapid, being able to control external and internal structure. It is also a reproducible process easy to shape. The scaffolds remain highly porous, $65 \%$ of porosity and with interconnected pores of around $500 \mu \mathrm{m}$ and $600 \mu \mathrm{m}$ wall thickness. It is possible to create smaller pores but decreasing the porosity. The elastic modulus observed for a porosity of $65 \%$ with square pores is around $6 \mathrm{MPa}$. One of the biggest disadvantages responsible for the rejection of this technique is its high cost due to the need for high technological equipment (144-146).

Electrospinning is an easy and economical technique used to fabricate porous samples. The electrospinning equipment uses a high voltage supplier, a capillary tube with small diameter 
extremity, containing a polymer solution and a metal collector. The high voltage is employed to create a charged jet of polymer solution out of the tube, which accelerates, thins and dries in the electric filed, ultimately collecting on a grounded device. The use of high voltage can represent labour risk. The material formed are nanoscaled fibres of polymer, these fibres superposed create the scaffold. This scaffold possess high surface to volume ratio that promotes cell attachment. However the pore size obtained of the interfibres is around 5$10 \mu \mathrm{m}$ which is too small to permit cell penetration even though the pores are interconnected. Furthermore the mechanical properties are not recommendable for load support as the network of fibres lack rigidity, and most of them present elastic modulus around $200 \mathrm{kPa}$. Usually the scaffolds of electrospun PLLA fibres have a $2 \mathrm{D}$ application such as membranes support for cell in the case of skin or heart tissue engineering (147-150).

Moulding techniques permit to modulate scaffold pore size and porosity. This technique involves filling a mould with polymer powder and then heating the mould above the glass transition temperature of polymer while applying pressure to the mixture; in this process a porogen template is performed by sintering the polymer powder. Compressive moulding (also known as melt injection moulding) requires the application of high processing temperature. The polymer is inserted to a mould, filled with the porogen template (previously shaped), in melting phase. The temperature of processing PLLA for melt injection moulding is around $180^{\circ} \mathrm{C}$. This may suppose a problem in the selection of an adequate porogen, as the template needs to be such so it does not deform at that temperature. Alternative materials for the template would be polyethylene oxide (PEO) which has a flash point from $180^{\circ} \mathrm{C}$ to $280^{\circ} \mathrm{C}$ or chloride magnesium which has a degradation temperature of $300^{\circ} \mathrm{C}$. In order to avoid such high processing temperatures, solution injection moulding can be another 
option. The polymer is inserted to the mould dissolved in a solvent, with the aid of compression pressure. This technique permits to fabricate highly porous scaffold $(90 \%)(151,152)$.

Freeze drying is one technique used to fabricate PLLA porous scaffolds, involving the removal of a solvent first by sublimation of a frozen sample and then by desorption of the non-frozen sorbed solvent under reduced pressure or by solvent leaching. In this case the main inconvenience is the use of a freeze dryer instrument. A similar technique able to generate highly porous PLLA scaffolds which does not need the use of a freeze dryer is the freeze-extraction process. It consists of the elimination of the solvent by dissolution rather than sublimation at polymer solution freezing state, which permits a gain on energy and time. Freeze-extraction combined with particle leaching is a simple, low cost and reproducible technique to fabricate highly porous scaffolds with interconnected pores giving the scaffolds suitable mechanical properties. The resulting porosity is double, micro and macro; micropores are formed from the leaching of the solvent and macropores from the leaching of the porogen. Freeze-extraction allows addition of inorganic components to the scaffolds by previous mixing nanoparticles with the polymer solution, in this way physiological or mechanical properties are increased $(55,153-156)$. In this process the temperature of polymer solution is decreased to induce phase separation into two phases, one having a high polymer concentration and one having a low polymer concentration or normally pure solvent crystallized phase.

Polymer-solvent phase separation can be divided into liquidliquid phase separation, which may occur prior to the solvent freezing and solid-liquid phase separation, which is only observed when the solvent has been completely frozen. The addition of a non-solvent to the polymer solution may induce liquid-liquid phase separation. Two types of liquid-liquid phase separation can occur when a homogeneous polymer 
solution is cooled. Between the binodal and spinodal curves of the phase diagram, the liquid-liquid phase separation proceeds through a nucleation and growth mechanism and gives rise to a generally dispersed structure. In contrast, below the spinodal curve, the original solution undergoes a spontaneous liquid-liquid phase separation referred to as the spinodal decomposition, with the formation of an interconnected network of two phases. In the case where the solvent crystallizes before liquid-liquid phase separation occurs, this solid-liquid phase separation is responsible for a final morphology that depends on the crystallization mode of the solvent. Schugens et al. studied that no liquid-liquid phase separation is observed when solutions of polylactides in 1,4dioxane are cooled, at least until a solid-liquid phase separation occurs due to solvent crystallization. Which corresponds to the case where the critical solution temperature of liquid-liquid phase separation is largely lower than the freezing point of the solvent $(157,158)$.

\subsubsection{Bioactive poly(L-lactic acid)/inorganic composites}

Poly(L-lactic acid) and bioactive ceramics are being combined in a variety of composite materials for bone tissue engineering scaffolds. Around 60 wt.\% of bone is made of HAp, Ca 10 $\left(\mathrm{PO}_{4}\right)_{6}(\mathrm{OH})_{2}$, and therefore it is evident why HAp and related calcium phosphates (e. g. $\alpha$-TCP, $\beta$-TCP $(101,159)$ have been intensively investigated as the major component of scaffold materials for bone tissue engineering. As expected, calcium phosphates have an excellent biocompatibility due to their close chemical and crystal resemblance to bone mineral. If the properties of HAp and related calcium phosphates are compared with those of cortical bone, bone has a reasonably good compressive strength though it is lower than that of HAp. However, bone has significantly higher fracture toughness than HAp. The high tensile strength and fracture 
toughness of bone are attributed to the tough and flexible collagen fibers reinforced by HAp crystals. Hence, calcium phosphates alone cannot be used for load-bearing scaffolds despite their good biocompatibility and osteoconductivity (72). The composition of bioactive particles with polymers is an option to the pure ceramic scaffolds. Most of the ceramics used to fabricate composite scaffolds of PLLA are nanoparticles of HAp. It was possible to fabricate highly porous composites increasing the bioactive potential of the PLLA and the mechanical properties. The nanoparticles can be in needlelike shape or more spherical $(160,161)$. The particles of HAp are supposed to transmit resistance to the polymer matrix. This transmission of resistance depends on the adhesion between polymer and particles. In the case of internal interfaces with poor adhesion it might provide links to nucleation of cracks. As the superficial nature of PLLA and HAp does not permit a chemical bond there is a weak adhesion between the two substances. In order to improve the interfacial connection of hydroxyapatite to PLLA Wang et al. used silane to modify the surface of the nanoparticles of HAp, and so increase the mechanical properties of the PLLA/ HAp composites and also to prevent aggregation of HAp powder. The interactions between silane modified HAp particles surface and hydroxyl or carboxyl groups of PLLA is stronger as HAp instead of showing up HAp-P-OH shows HAp-P-OH$\mathrm{Si}(\mathrm{OH})_{2}-\left(\mathrm{CH}_{2}\right)_{17}-\mathrm{CH}_{3}$. The best increase in terms of elastic modulus was obtained for scaffolds filled with $20 \%$ of modified HAp from 0.65 to $0.83 \mathrm{MPa}$, with fibers about $100-800 \mu \mathrm{m}$ and 93.6\% porosity, the scaffolds dimension being $20.5 \mathrm{~mm}$ diameter and $5.0 \mathrm{~mm}$ high. For the scaffolds made by freezedrying with $100-300 \mu \mathrm{m}$ pore dimension elastic modulus increased from 2.14 to $6.44 \mathrm{MPa}$ for the scaffolds filled with $15 \%$ of modified HAp particles in comparison to that not modified $(162,163)$. Other representative bioactive ceramics used to fabricate porous PLLA composite scaffolds are 
bioactive glass. In these cases the elastic modulus could be increased from 5.5 to $8.0 \mathrm{MPa}$ for the scaffolds with cubic form $(10 \times 10 \times 10 \mathrm{~mm})$ and $89 \%$ porosity filled with $30 \%$ of bioactive bioglass. When scaffolds filled with bioactive glass are previously treated with silane there is an increase in the elastic modulus of up to $80 \%$. The elastic modulus increased from 107.7 to 178.6 and up to $259.6 \mathrm{MPa}$ from the scaffolds not filled, the ones filled with $29 \mathrm{vol} \%$. of bioactive glass and the ones filled with 29 vol\%. modified bioactive glass in the works of Zhang and Hong $(101,103)$. 


\section{Chapter 2. Materials and methods}

In this chapter the techniques used to prepare and characterise the materials involved in this work will be described. As a first step porous scaffolds of poly(L-lactic acid) (PLLA) with different porous morphologies have been prepared and studied. From this evaluation a composition of the reactive mixture to prepare the porous scaffolds was selected to design the scaffold for the cartilage layer of the osteochondral construct. Then evolution of the physical and chemical properties under static and dynamic degradation of this scaffold was studied. In order to bioactivate the material for the bone anchorage, hybrid composite structures in $2 \mathrm{D}$ and 3D of PLLA/ bioactive particles were fabricated. The influence of the content and the nature of the bioactive particles on the properties of the samples were studied. Finally the osteochondral construct was assembled.

\section{$2.1 \quad$ MATERIALS}

\subsubsection{Micro and macro porous scaffolds.}

Micro and macroporosity are one of the main morphological qualities for scaffolds fabricated in this work. The macropore structure should host seeded cells and host the regenerated tissue while the network of microchannels allows the diffusion of water and soluble substances through the scaffold. With the variation and combination of these two morphological aspects physical-chemical properties of the scaffolds can be modified over a broad range. This part deals with the design of different morphologies for the selection of the adequate for our application. The techniques used to prepare these samples were freeze extraction and particle leaching leading to the 
formation of micro and macropores, respectively. The design of these scaffolds combines the effects of two different parameters as polymer/ solvent concentration and polymer/ porogen ratio. The poly lactic acids used were from the commercial brands Cargill Dow (Minneapolis, USA) and Purac (Barcelona, Spain). The Cargill Dow lactic acid is of industrial use. It contains a $99.6 \%$ L-lactide isomer, its average molecular weight is $124 \mathrm{kDa}$, with a polydispersity index $\mathrm{Mw} / \mathrm{Mn}=1.83$ (155). The PLLA from Purac (Purasorb PL 18) is a medical grade polymer with a viscosity of $1.8 \mathrm{dl} / \mathrm{g}$. 1,4Dioxane, commonly called dioxane, has been chosen for the freeze extraction process because of its capacity to dissolve PLLA and its high melting point that permits its extraction in solid state when it is at $-10^{\circ} \mathrm{C}$. It was purchased from Sharlau Chemie, S.A. (Barcelona, Spain) and used without any purification. The porogen used is Elvacite®2043, a low molecular weight acrylic resin (low molecular weight poly(ethyl methacrylate) (PEMA) in the form of spherical beads, produced by Lucite International (Southampton, UK). It has an average molecular weight of $50 \mathrm{kDa}$. The diameter of the beads is around $200 \mu \mathrm{m}$, with low dispersion (140-220 $\mu \mathrm{m}$ as claimed by the producer). In order to assure the dimension of the porogen, the particles of PEMA were sieved before their use, in that way particles below $53 \mu \mathrm{m}$ were discarded. This material was chosen as porogen because of its solubility in ethanol; ethanol is in fact one of the few solvents, that does not dissolve PLLA.

In order to vary the polymer concentration, a series of poly(Llactic) acid/ dioxane solutions with 5, 10, 15, 18 and $20 \%$ wt. of polymer content were used to prepare different scaffolds. The obtained scaffolds will be called (5/95), (10/90), (15/85), (18/82) and (20/80) respectively, corresponding to the (PLLA/ dioxane) content. The different PLLA solutions were mixed with two different polymer solution and porogen ratios, 1:1 
and 1:1.25 (w/w), with the aim to vary the polymer / porogen ratio. In the text the different samples are named 1:1-(5/95), (10/90), (15/85), (18/82), (20/80) and 1:1.25-(5/95), (10/90), (15/85), (18/82), (20/80) scaffolds.

Consequently the five different PLLA solutions were poured into Teflon moulds, quickly mixed with the PEMA porogen with a spatula, and then immediately frozen in liquid nitrogen. Then, cold ethanol at $-10^{\circ} \mathrm{C}$ was poured on the frozen mixtures in order to dissolve the crystallized dioxane. Dioxane extraction was conducted in a cold ethanol bath at $-10^{\circ} \mathrm{C}$, ethanol was changed until the dioxane had completely disappeared from the structure, and so leads to the formation of the micropores. It is important not to increase the temperature up to the melting temperature of dioxane $\left(11.8^{\circ} \mathrm{C}\right)$ until the dioxane has been totally removed as it could either dissolve the PLLA or swell the particles of porogen and so deform the porous structure. Afterwards, the extraction of the porogen with hot ethanol at $40^{\circ} \mathrm{C}$ and slow stirring was done; ethanol was changed until the PEMA had completely disappeared from the structure, leading to the formation of the macropores. After extraction, scaffolds were dried firstly at room temperature for 24 hours, then under vacuum for 24 hours and then under vacuum at $40^{\circ} \mathrm{C}$ until constant weight. The scaffolds were cut into cylinders of $6 \mathrm{~mm}$ diameter and 4 mm high.

This part of the study led us to the selection of a scaffold formulation which resulted in the best candidate for the cartilage part of the osteochondral construct, after the evaluation of the influence of the macro and micro porosity on the scaffold properties. 


\subsubsection{Hybrid scaffolds PLLA/bioactive particles}

The bioactivity and mechanical properties of the scaffolds were planned to be enhanced incorporating bioactive nanoparticles, such as HAp and $\mathrm{SiO}_{2}$, to the polymeric structure. First this study involved membranes of the samples. Varying the content of the filler it was possible to study the influence of the amount of nanoparticles to the bioactivity of the material and the properties of the membraness.

\subsubsection{Fabrication of the 2D hybrid structure}

A series of poly(L-lactic acid)/ hydroxyapatite composite membranes with HAp content 0, 5, 10 and 15\% (w/w) were prepared by freeze extraction method. The fabrication process is the same as used in section 2.1.1, p.54, to fabricate scaffolds but the incorporation of porogen and particle leaching processes was skipped as we intended to produce flat samples and without macropores. Hydroxyapatite particles with particle size inferior to $200 \mathrm{~nm}$ were purchased from SigmaAldrich (Spain); the nanoparticles of HAp were sieved before incorporation to the solvent. PLLA for industrial use was used for this study. The required quantity of HAp powder was dispersed in dioxane by sonication, then a $15 \%$ weight PLLA (relative to solvent weight) was added to the mixtures, and stirred until completely dissolved. As mentioned above, four solutions with different content in HAp were prepared with 0 , 5, 10 and $15 \%$ particles, this percentage is in regard to PLLA weight. Then the membranes were fabricated by freeze extraction process. The membranes were cut into squares (1 $\mathrm{cm} \times 1 \mathrm{~cm})$ of $200 \mu \mathrm{m}$ thickness. To obtain the desired thickness the samples were shaped in compound resin (O.C.T.TM) from Tissue-Tek at $-30^{\circ} \mathrm{C}$ and cut by cryogenic microtome. After removing residues of the resin, samples were dried during three days. The first day at room temperature, the second under vacuum and the third under vacuum at $40^{\circ} \mathrm{C}$. 


\subsubsection{Fabrication of the 3D hybrid structure}

The same type of composites as in the previous section were made but, in this case, in three dimension by adding the particle leaching step to the freeze extraction process. After confirming that the HAp was not as effective as expected for the enhancement of the mechanical properties of the 3D PLLA scaffolds, different inorganic filler based on silica particles was introduced.

\section{PLLA/HAp COMPOSITES}

A composite scaffold of poly(L-lactic acid)/ hydroxyapatite with a $5 \%$ wt. of HAp was prepared by the freeze extraction and particle leaching processes following the fabrication procedure as presented in section 2.1.1, p.54. A pure organic scaffold was prepared as a reference material. The samples will be named PLLA and PLLA/ HAp scaffolds. The HAp nanoparticles were sieved before incorporation to the solvent. Briefly, the required quantity of HAp powder was dispersed in dioxane by sonication, then a $15 \%$ weight PLLA was added to the mixture, and stirred until completely dissolved. The PLLA and the PLLA/ HAp solutions were poured into Teflon moulds, quickly mixed with the PEMA porogen with a spatula, and then immediately frozen in liquid nitrogen. Then, cold ethanol at $-10^{\circ} \mathrm{C}$ was poured on the frozen mixtures in order to dissolve the crystallized dioxane. Dioxane extraction was conducted in a cold ethanol bath at $-10^{\circ} \mathrm{C}$, ethanol was changed until the dioxane had completely disappeared from the structure. Afterwards the extraction of the porogen with hot ethanol at $40^{\circ} \mathrm{C}$ and slow stirring was done; ethanol was changed until the PEMA had completely disappeared. The scaffolds were cut into cylinders of $6 \mathrm{~mm}$ diameter and $4 \mathrm{~mm}$ high. Samples were dried firstly at room temperature for 24 hours and then under vacuum for 24 hours and then under vacuum at $40^{\circ} \mathrm{C}$ for 24 hours more. 
Unfortunately this fabrication process did not enhance the mechanical properties of the scaffolds. Two strategies were followed to improve the mechanical properties of the organic matrix:

- A second sonication process was employed after dissolving the PLLA in the dioxane/ particles mixture and before adding the PEMA porogen.

- The increase of HAp nanoparticles content $(5,10,15$, 18, 20 wt.\%).

- The combination of HAp particles with finer inorganic particles based on nanoporous silicates with the aim to enhance the interaction area of the filler and the organic matrix, which could lead to an increase of the mechanical properties.

\section{PLLA/ HAP/ $\mathrm{SIO}_{2}$ COMPOSITES}

The filler used to fabricate these composites consisted of a mixture of the same HAp particles as before, previously sieved, and laboratory made particles of silicates. The silicate particles were nanoparticulated bimodal porous silicas (NBSs) with particles size of 20-30 nm; they were purchased from the Materials Science Institute of the University of Valencia (164). A series of scaffolds were prepared with (100/0/0), (95/0/5), (95/5/0), (90/10/0) and (90/9.5/0.5) \% wt. of PLLA/ HAp/ $\mathrm{SiO}_{2}$ respectively. Freeze extraction and particle leaching, the same procedure explained in section 2.1 .1$, p.54, were used to fabricate these $3 \mathrm{D}$ composites. In order to increase the dispersion of the particles in the polymeric matrix double sonication was applied; when the nanoparticles were added to the dioxane and after the PLLA, added to the HAp/ $\mathrm{SiO}_{2} /$ dioxane mixture, was dissolved (prior to pour the PEMA porogen). The scaffolds were cut into cylinders of $6 \mathrm{~mm}$ diameter and $4 \mathrm{~mm}$ high. Samples were dried first at room temperature for 24 hours and then under vacuum 24 hours and then under vacuum at $40^{\circ} \mathrm{C}$ for 24 hours more. 


\subsubsection{Bioactivity test}

The in vitro bioactivity test was assessed as specified in section 1.3 , p.30, to determine the ability of the samples to precipitate HAp from the ions in a SBF solution while they are in immersion. This layer was characterised to find out the type of HAp coating on the surface of the samples. SBF solution was prepared using the Müller method (95) from different volumes of eight salt solutions of $\mathrm{KCl}$ (59.64 g/l), $\mathrm{NaCl}$ (116.88 g/l), $\mathrm{NaHCO}_{3}(45.37 \mathrm{~g} / \mathrm{l}), \quad \mathrm{MgSO}_{4} .7 \mathrm{H}_{2} \mathrm{O}(49.30 \mathrm{~g} / \mathrm{l}), \mathrm{CaCl}_{2}$ (14.702 g/l), TRIS (tris-hydroxymethyl aminomethane; 121.16 $\mathrm{g} / \mathrm{l}$ ) (used for $\mathrm{pH}$ adjustment, see below), $\mathrm{NaN}_{3}(1 \mathrm{~g} / \mathrm{l}), \mathrm{KH}_{2} \mathrm{PO}_{4}$ $(27.22 \mathrm{~g} / \mathrm{l})$ poured into $650 \mathrm{ml}$ deionised ultra-pure water, in order to prevent salt precipitation and maintain the $\mathrm{pH}$ of the solution. The exact volume in millilitres of the different solutions used to fabricate the SBF (added to the $650 \mathrm{ml}$ of water) is detailed in the Table 2-1. Then the flask containing the salts was filled with deionised ultra-pure water up to 1000 $\mathrm{ml}$. For $\mathrm{pH}$ adjustment to $\mathrm{pH}$ 7.6-7.7 at $25^{\circ} \mathrm{C}$ (which corresponds to $\mathrm{pH} 7.3-7.4$ at $\left.37^{\circ} \mathrm{C}\right), \mathrm{HCl}$ or TRIS was added.

Table 2-1: Volume of salt solutions used to prepare $1 \mathrm{~L}$ of SBF.

\begin{tabular}{lllllllll}
\hline Salts & $\mathrm{KCl}$ & $\mathrm{NaCl}$ & $\mathrm{NaHCO}_{3}$ & $\mathrm{MgSO}_{4} \cdot 7 \mathrm{H}_{2} \mathrm{O}$ & $\mathrm{CaCl}$ & Tris. $\mathrm{HCl}$ & $\mathrm{NaN}_{3}$ & $\mathrm{KH}_{2} \mathrm{PO}_{4}$ \\
\hline $\begin{array}{l}\text { volume } \\
(\mathrm{ml})\end{array}$ & 5 & 60 & 10 & 5 & 25 & 50 & 10 & 5 \\
\hline
\end{tabular}

Samples were soaked in SBF for different times up to 21 days. The SBF solution was not renewed during the first 7 days. Afterwards, the ion concentrations were adjusted to twice those of SBF (2xSBF) and the solution was renewed each 2-3 days, in order to provide more favourable conditions for apatite deposition. As the hydrophobic characteristic of PLLA hindered its immersion in the biological fluid, the pores of the scaffolds were forced to fill of SBF with vacuum. The minimum volume of SBF used for testing the bioactivity of the 
samples followed the recommendation that it should be above the $S_{a} / 10$, where $S_{a}$ is the apparent surface area (93).

\subsubsection{Oxygen plasma treatment}

Surface modification is one method presented in section 1.1.6 to increase the bioactivity of a polymer. Surface modification of some materials was carried out before SBF immersion in order to form hydrophilic groups on the surface of the material which tend to favour the precipitation of ions in presence of the medium responsible for the coating of a layer of HAp (121). The plasma treatment was applied with oxygen plasma (Plasma Electronic, model Piccolo, quartz cylinder, $2.45 \mathrm{GHz}$ generator) at $300 \mathrm{~W}$ in a $50 \mathrm{~Pa}$ vacuum, for 120 seconds for each side. The samples were used for experiments within 1 hour after exposure, time enough for the radicals recombination on the surface of the samples.

\subsubsection{Sonication}

The dispersion of the nanoparticles in the polymeric matrix was done by sonication using a GM 3200 Sonoplus Ultrasonic homogenizers from Bandelin (Berlin, Germany). The sonication was applied during five minutes in pulsation mode ( 2 seconds ON, 1 second $\mathrm{OFF}$ ), at $50 \%$ of $200 \mathrm{~W}$, and at a frequency of $20 \mathrm{kHz}$. In order to avoid heating of the solution, the flask was immersed in cold water inside a Teflon mould with ice cubes.

\subsubsection{Static degradation}

The evolution of the properties of the 1:1-15\%-PLLA-MED scaffold under in vitro static degradation were analysed. This study was done in collaboration with the Group of Structural Mechanics and Materials Modelling, of the University of Zaragoza, in Spain (under the supervision of Drs. Manuel 
Doblaré and Ignacio Ochoa and as part of the $\mathrm{PhD}$ thesis of Dr. Victor Acosta (165). Scaffolds were shaped in cylinder of 6 $\mathrm{mm}$ diameter and $4 \mathrm{~mm}$ thick. Then 65 samples were immersed in phosphate buffered saline solution, PBS, each one in $2 \mathrm{~mL}$ at $37^{\circ} \mathrm{C}$. The immersion times for this study were $0,6,12,18$ and 24 weeks. Furthermore 2 samples were subjected to static degradation during 1 year. The PBS bath was renewed every 2 weeks. After the different times of immersion in PBS the scaffolds were analysed by several tests to see the influence of degradation time on the properties of the scaffolds.

\subsubsection{Fatigue essay}

The 1:1-15\%-PLLA-MED scaffolds were also subjected to degradation under dynamic compression conditions. In order to perform the fatigue essay blocks of Teflon were fixed on the extremity of the arms of the compression machine (Microtest Universal Testing Machine) to avoid their oxidation. The upper side was used to apply the compression, and the lower side was filled with PBS and the scaffolds were placed there, properly ordered to identify each of them. A PBS flow was supplied by a peristaltic pump at $2 \mathrm{ml} / \mathrm{min}$. In order to maintain the PBS temperature at $37^{\circ} \mathrm{C}$ a thermostatic water bath at $40^{\circ} \mathrm{C}$ was used. The silicon PBS circulating tube was immersed in the water to warm the fluid. Silicon tubes with 4 $\mathrm{mm}$ external diameter and $0.8 \mathrm{~mm}$ internal diameter were used for the PBS flow. The circuit can be seen on the photo Figure 2-1. 


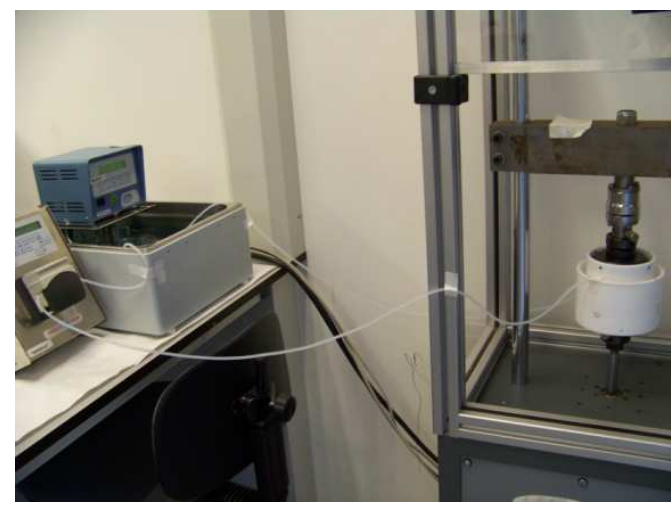

Figure 2-1: Fatigue test circuit.

The tested scaffold was the same as that used for the static degradation; in cylinder shape $6 \mathrm{~mm}$ diameter and $3 \mathrm{~mm}$ thick. The applied strain was $5 \%$ in compression with a predeformation of $0.25 \%$, at $1 \mathrm{~Hz}$. The scaffolds were tested during several times: 1, 2, 4 and 6 weeks. The PBS was renewed every week. At each degradation time the scaffolds were removed from the bath, dried and analysed by several tests to see the influence of degradation time under dynamic conditions on the properties of the scaffolds

\subsubsection{Biconstruct fabrication: bilayered scaffold for osteochondral regeneration}

The aim of this work was to fabricate a bilayered construct with an impermeable junction between each macroporous part. The biconstruct is the combination of two scaffolds fabricated by freeze extraction and particle leaching. The scaffold formulation used in the fabrication of the cartilage part of the biconstruct resulted from the study of the influence of micro and macroporosity on the scaffold's properties. The formulation used to the fabrication of the bony part of the biconstruct was a consequence of the results obtained after filling the scaffold with HAp particles. Thus, the up side of the biconstruct aimed at cartilage repair was a 1:1-15\%PLLA- 
MED scaffold, $1 \mathrm{~mm}$ high (1:1-15\%PLLA refers to a scaffold fabricated with the same mass ratio of porogen and PLLA/dioxane solution, containing this solution a $15 \% \mathrm{wt}$. of PLLA). The lower side was similar to the upper side (1:1$15 \%$ PLLA-MED scaffold), but functionalised for bone regeneration in the sense that it was fabricated with a $5 \%$ of bioactive HAp nanoparticles; and it was $6 \mathrm{~mm}$ high. So the biconstruct was a sandwich of (100/0) and (95/5)- 1:1$15 \%$ PLLA-MED scaffold. The two parts were separately prepared, and once the porogen was removed and the samples were dried they were joined by applying a thin layer of PLLA solution in dioxane of $15 \%$ wt. of PLLA. The purpose of this layer is to prevent blood flow from the bone to the cartilage area once the construct is implanted. In order to remove the dioxane the bilayered construct was dried during $24 \mathrm{~h}$ at $40^{\circ} \mathrm{C}$.

\subsection{CHARACTERIZATION TECHNIQUES}

\subsubsection{Scanning Electron Microscopy (SEM)}

The morphology was characterised by scanning electron microscopy (SEM) analysis, with a JOEL JSM 6300 scanning electron microscope in the secondary mode under an acceleration tension of $15 \mathrm{kV}$ and working distance $15 \mathrm{~mm}$. The cross-section surface of each sample was sputter coated with a gold layer under vacuum to ensure conduction. An estimation of the pores sizes was determined by direct 3 measurements of both micro and macropores from 3 SEM pictures for each composition. 


\subsubsection{Elemental analysis (EDS)}

For EDS analysis, samples were mounted onto copper holders and carbon sputtered. Elements quantification was performed by Electron Dispersive Spectroscopy (EDS) with a JEOL JSM 6300 scanning electron microscope with an acceleration tension of $10 \mathrm{kV}$.

\subsubsection{Porosity}

Porosity was measured by a gravimetric method. Firstly the dry samples were weighed, and then filled with ethanol under vacuum, and subsequently weighed. Porosity was calculated as the quotient of the volume of pores and the total volume of the scaffold. The volume of pores, $V_{\text {pore }}$, was deduced from the weight difference between the dry $\left(m_{d r y}\right)$ and wet $\left(m_{w e t}\right)$ sample, according to equation (3) - assuming that the amount of ethanol absorbed by the polymer matrix is negligible during the short time of the experiment

$$
V_{\text {pore }}=\frac{m_{w e t}-m_{d r y}}{\rho_{\text {eth } O H}},
$$

where $\rho_{\text {ethoH }}$ is the density of ethanol.

Thus, the volume of the pores equals the volume occupied by the absorbed ethanol.

The total volume (equation 4) is the volume occupied by the pores and that occupied by the polymer. The volume of the polymer is:

$$
V_{\text {polymer }}=\frac{m_{d r y}}{\rho_{\text {polymer }}} .
$$

So the porosity $(\mathrm{P})$ is done by the equation 5 : 


$$
P=\frac{\frac{m_{\text {wet }}-m_{d r y}}{\rho_{\text {ethanol }}}}{\frac{m_{\text {wet }}-m_{d r y}}{\rho_{\text {ethanol }}}+\frac{m_{d r y}}{\rho_{\text {polymer }}}} \cdot 100 .
$$

At least five measurements were carried out for each sample. The volume occupied by the polymer was calculated from the dry weight of the scaffold assuming a density of PLLA of 1.19 g.cm ${ }^{-3}(155)$.

\subsubsection{Compressive test}

In order to study the mechanical properties of the samples, a compression test was done, using a Microtest Universal Testing Machine. A compression with a constant speed of 1 $\mathrm{mm} / \mathrm{min}$ until $500 \mathrm{~N}$ was applied on the dry scaffolds, at room temperature. For each scaffold the test was repeated five times for statistical analysis. In Figure 2-2 a schematic compressive stress-strain curve for a PLLA foam is shown. The stress was calculated by the delivered force divided by the initial area of the scaffold and the strain was determined by dividing the displacement of the gag by the initial sample height. The graph shows a linear elastic deformation at low stresses, followed by a long collapse plateau, truncated by a regime of densification in which the stress rises steeply (166). Linear elasticity is related to cell wall bending, and the apparent compressive elastic modulus, $E_{\text {elastic, }}$ is the initial slope of the stress-strain curve; the plateau (characterized by yield point) is associated with the collapse of the cells by elastic buckling; finally, when the cells have almost completely collapsed, opposing cell walls touch and further strain compresses the solid itself, giving rise to the final region of rapidly increasing stress; the densification zone (characterized by densification point). 


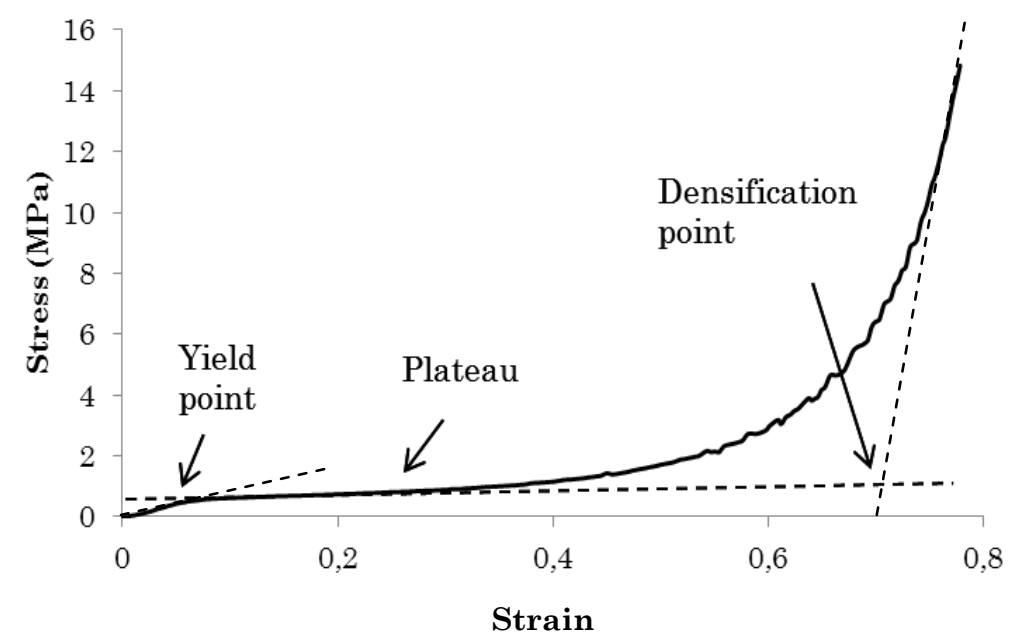

Figure 2-2: Typical regimes of compressive stress-strain curves of polymeric foam. A real measurement on a PLLA scaffold has been used as illustration.

The compressive test used to study the mechanical properties of the scaffolds after static degradation were realised in collaboration with the the Group of Structural Mechanics and Materials Modelling, of the University of Zaragoza, in Spain. The protocol involved in this study was different from the previous one. A slower compression, with a constant speed deformation of $0.01 \mathrm{~mm} / \mathrm{s}(0.6 \mathrm{~mm} / \mathrm{min})$ until $50 \mathrm{~N}$, was applied on the scaffolds, at room temperature and immersed in a PBS solution in a Instron MicroTester 5548 machine. A pre-deformation of a $4 \%$ the initial height was applied to standardize the experiments. The samples were conditioned 24 hours before the experiment by their immersion in SBF. For each scaffold the test was repeated five times for statistical analysis. 


\subsubsection{Dynamic mechanical spectroscopy (DMS)}

The dynamic mechanical properties of the membranes (2D hybrids) were determined by the Seiko Instruments Extrar6000 Thermal Analysis and Rheology System. The samples were heated first from $0^{\circ} \mathrm{C}$ up to $120^{\circ} \mathrm{C}$ at $2^{\circ} \mathrm{C} / \mathrm{min}$, maintained at $120^{\circ} \mathrm{C}$ for 60 minutes, and then cooled to $0^{\circ} \mathrm{C}$ at $2^{\circ} \mathrm{C} / \mathrm{min}$ and heated again to $150^{\circ} \mathrm{C}$ at the same rate. Testing frequency was $1 \mathrm{~Hz}$ and the membranes presented rectangular dimensions $3 \times 3 \times 30 \mathrm{~mm}^{3}$.

\subsubsection{Differential scanning calorimetry (DSC)}

Thermal transitions were evaluated by thermal analysis with the PERKIN ELMER differential scanning calorimeter Pyris I. Each sample was underwent to two heating cycles from $0^{\circ} \mathrm{C}$ to $210^{\circ} \mathrm{C}$ at a heating rate of $10^{\circ} \mathrm{C} / \mathrm{min}$, between the two heating scans there was a cooling scan from $210^{\circ} \mathrm{C}$ to $0^{\circ} \mathrm{C}$ at a heating rate of $10^{\circ} \mathrm{C} / \mathrm{min}$.

\subsubsection{Gel permeation chromatography (GPC)}

The molecular weight of the samples was determined with gel permeation chromatography at $30^{\circ} \mathrm{C}$, using a Water Breeze GPC system equipped with a 2414 refractive index detector and Styrage HR THF columns. Tetrahydrofuran (THF) was used as the eluent at a flow rate of $0.5 \mathrm{ml} / \mathrm{min}$. The calibration curve was prepared by using monodisperse polystyrene standards from Shodex.

\subsubsection{Thermogravimetric analysis (TGA)}

Thermogravimetric analysis (Thermogravimetric analyser SDTQ600 from TA Instruments) was used in order to determine the thermal degradation profile of the samples and the quantity of HAp or silica nanoparticles and biomimetic 
deposited HAp in the samples. The samples were subjected to a temperature ramp from room temperature until $800^{\circ} \mathrm{C}$ at $20^{\circ} \mathrm{C} / \mathrm{min}$ under nitrogen flow $(50 \mathrm{ml} / \mathrm{min})$.

\subsubsection{Calcination experiments}

The real content of HAp in the PLLA/HAp composites was determined by weighting membrane residues after calcination. Calcination was carried out in a Conatec HC-300 ceramic oven. For each composite, three samples were heated up to $600^{\circ} \mathrm{C}$ at $10^{\circ} \mathrm{C} / \mathrm{min}$, and maintained at $600^{\circ} \mathrm{C}$ for 10 minutes, in order to entirely thermally degrade the PLLA.

\subsubsection{Fourier transform infrared analysis (FTIR)}

Chemical surface changes due to the HAp nucleation were studied using FTIR Thermo Nicolet Nexus apparatus in a transmission mode. The spectra resulted from averages of 128 scans at $4 \mathrm{~cm}^{-1}$ resolution, between 650 and $4000 \mathrm{~cm}^{-1}$. The sample, as a finely divided powder, was mixed with potassium bromide from Sigma-Aldrich at a FTIR grade, in (1/99) \% wt. proportion, respectively. 


\section{Chapter 3. Results}

\subsection{SCAFFOLDS FABRICATION: PROCESSING, MORPHOLOGY AND PROPERTIES RELATIONSHIPS}

Two types of PLLA have been used in the study. A PLLA from PURAC for medical use, developed for use in animal models in collaboration with the group of Prof. F. Prosper at the Clínica Universidad de Navarra, was selected. In this way possible effect of low molecular weight substances retained in the material or other by-products of synthesis process could be discarded when analysing the in vivo performance of the scaffolds. Nevertheless the high cost of this material meant carrying out many initial trials and some of the experiments were done with a PLLA from Cargill-Dow, which receives extensive use in packaging industry. From the point of view of an eventual clinical study, any scaffold would need extensive purification and deep toxicity analysis due to the chemicals used in processing. PLLA for industrial use and for medical use will be referred to in this manuscript respectively as PLLA-CD and PLLA-MED. The behaviour of the two materials might be slightly different in several aspects due to differences in molecular weight and stereochemistry. The aim of this section is mainly to determine the influence of processing conditions on the morphology and properties of the scaffolds. Morphology, mechanical and thermal properties, and porosity of the scaffolds will be characterised. As a conclusion of this study the composition of the best construct for the cartilage part of the osteochondral construct will be selected. 


\subsubsection{Scaffold morphology}

The structures of the PLLA scaffolds obtained by freeze extraction and particle leaching processes are presented below. The SEM pictures are classified in order to show both types of PLLA, firstly the scaffolds made with industrial PLLA-CD and then the medical grade PLLA-MED. For each type of PLLA both 1:1 and 1:1.25 PLLA-solution: porogen mass proportions are presented in the images. Each series of images shows the scaffolds obtained with each of the five polymer concentration in the dioxane solution (5, 10, 15, 18 and $20 \%$ in weight). The tests carried out on the different samples examine the influence of factors such as the polymer concentration, the polymer/ porogen ratio and the PLLA type on the final morphology of the scaffolds.

\section{- $\quad$ PLLA-CD}

Figure 3-1 and Figure 3-2 show the SEM pictures of cross sections of the scaffolds made with industrial PLLA. The morphology of the scaffolds is composed of two different types of cells. Small closed-cells called microporosity, because of its microdimensions, which are uniformly distributed all over the surface of the scaffolds. The microcells are arranged in undefined axes. The solid material closes off the cell faces, leaving the external face open. Furthermore the material shows a porous structure of open macrocells forming the so called macroporosity. The walls of the macropores are completely covered by microcells. Macropores are uniformly distributed throughout the entire scaffold. These open macrocells connect each other through smaller diameter throats. The appearance of the resulting structure is that of a $3 \mathrm{D}$ cellular material with open pore structure forming an interconnected network which in many aspects looks like trabecular bone (166). Macroporosity presents some changes 
when the polymer concentration varies. The macrocells trabeculae are well defined with a uniform macroporosity when the scaffolds are prepared with solutions of intermediate PLLA concentration, particularly for both series, from 5 to $15 \%$ of PLLA content. Nevertheless, for the 1:1-(20/80) scaffold and 1:1.25-(5/95)/ (18/82)/ (20/80) scaffolds the trabeculae are not well defined in certain areas or completely broken. The porous structure is somehow collapsed and the macropores appear completely deformed in the case of the 1:1-(20/80) and 1:1.25-(5/95) scaffolds. Scaffolds 1:1.25-(18/82)/ (20/80) present a structure with defined macropores but with very thin trabeculae

While the polymer concentration changes a variation of the microporosity can be seen. Micropores are uniformly dispersed on the surface of the scaffolds except on the surface of the 1:1(20/80) scaffolds and 1:1.25-(18/82)/ (20/80) scaffolds, where the microporosity does not show up. It can be observed that the micropores get bigger when the polymer content decreases, for both 1:1 and 1:1.25 polymer/porogen ratio series. The influence of porogen proportion is observed in the trabeculae dimension. Greater is porogen proportion smaller are the trabeculae. 


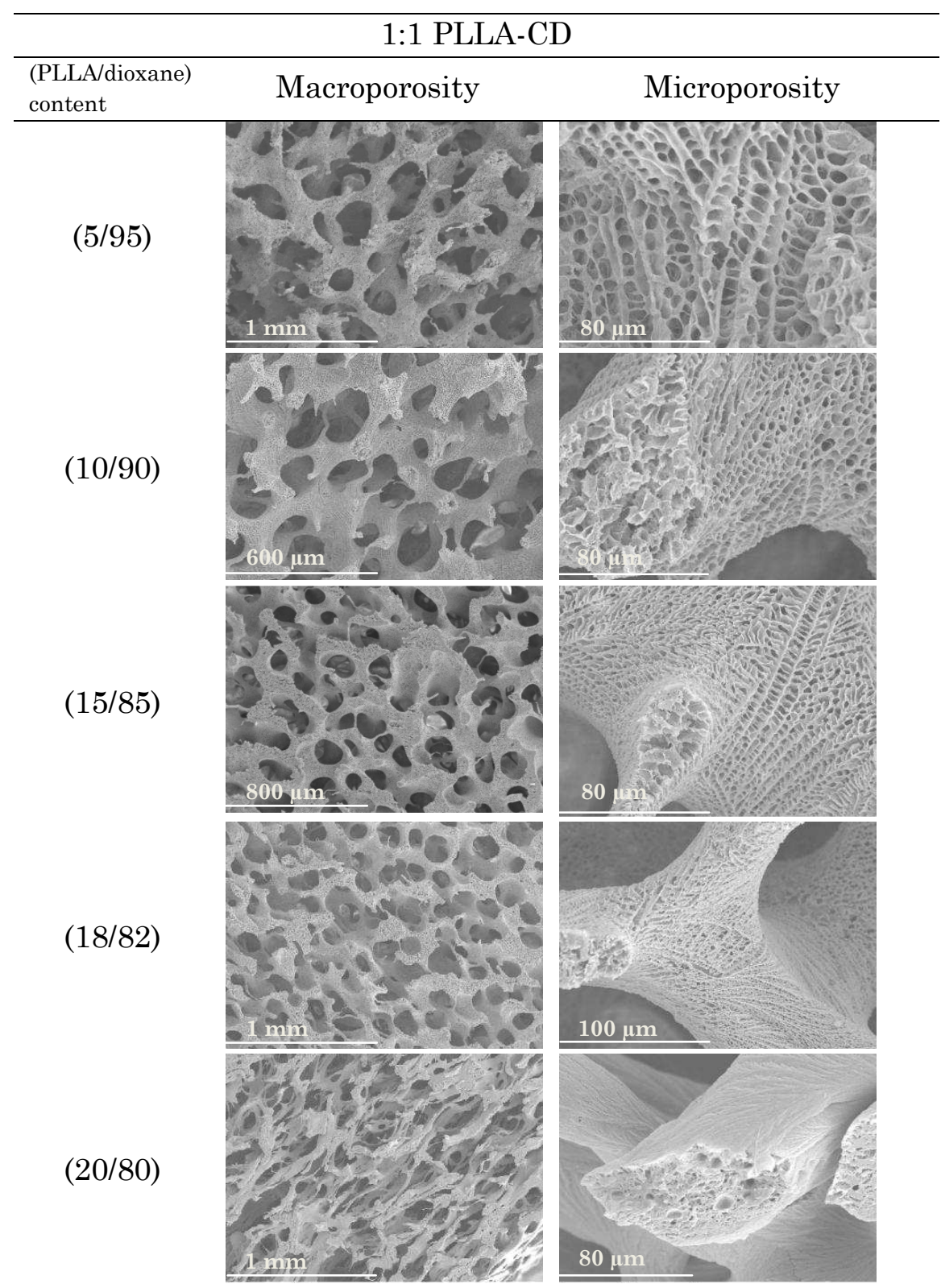

Figure 3-1: SEM pictures of 1:1-(5/95)/ (10/90)/ (15/85)/ (18/82)/ (20/80)-CD scaffolds 


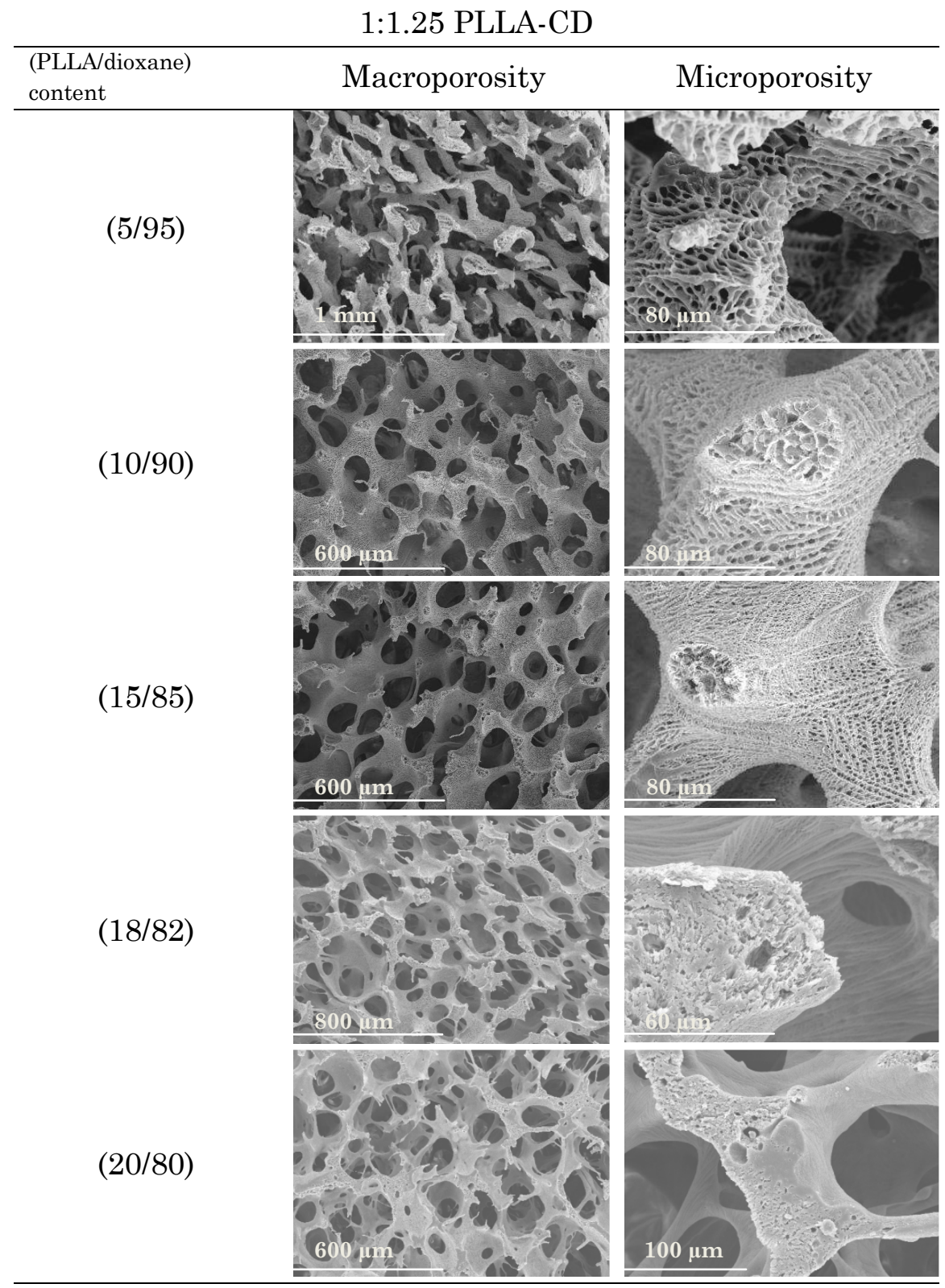

Figure 3-2: SEM pictures of 1:1.25-(5/95)/ (10/90)/ (15/85)/ (18/82)/ (20/80)-CD scaffolds. 
- $\quad$ PLLA-MED

The SEM pictures of the PLLA-MED scaffolds are presented in Figure 3-3 and Figure 3-4. The same morphologies as obtained in the case of industrial PLLA are observed. Interconnected macropores and micropores well dispersed on the surface of the pore walls can be identified. Defects on the structure caused by the polymer concentration, as broken trabeculae in some points, appear only for the 1:1.25-(20/80) scaffold. It can be seen that the trabeculae are getting thinner as the polymer concentration increases. This trend is better observed for the 1:1.25 series.

Micropores are well defined and regularly distributed on the macropore walls, except for the 1:1-(5/95)/ (20/80) scaffolds and $1: 1.25-(18 / 82) /(20 / 80)$ scaffolds. On the macropore walls of this last composition, (20/80), microcells form an undefined relief. 


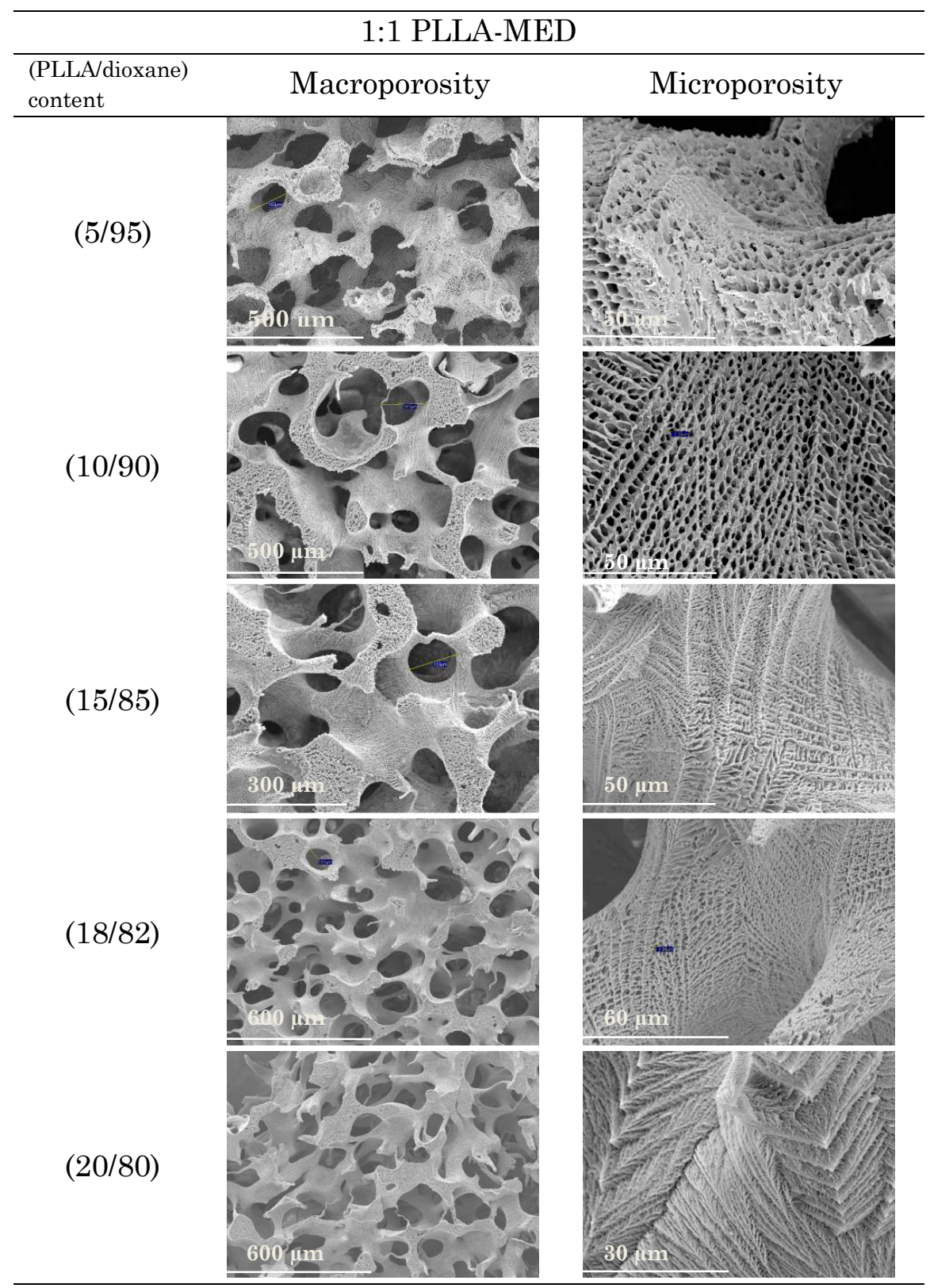

Figure 3-3: SEM pictures of 1:1-(5/95)/ (10/90)/ (15/85)/ (18/82)/ (20/80)-MED scaffolds. 


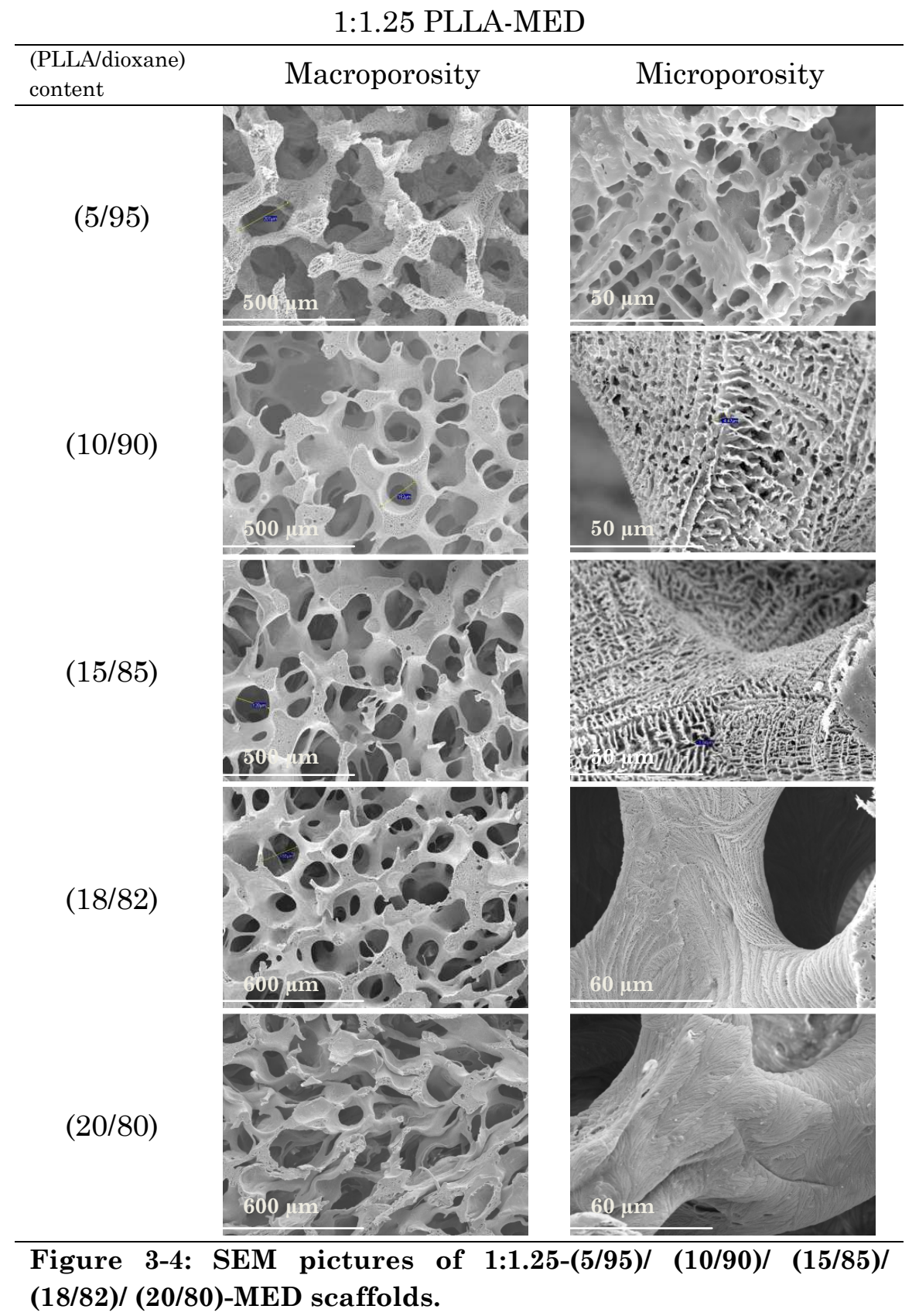




\subsubsection{Porosity}

Porosity, micropore and macropores dimensions of the different scaffolds are presented in Figure 3-5, Figure 3-6 and Figure 3-7 respectively. The porosity was determined as explained in section 2.2 .3 , p.58, the pores dimensions were directly determined from the SEM pictures. An average was made measuring five pores of three different scaffolds for both types of pores. Micro and macropores were both considered to determine the porosity.

A slight decrease of the porosity can be seen when the polymer concentration increases. The porosity varies from $81 \%$ to $96 \%$. The highest macropore dimensions are for the (5/95) scaffolds in both series; around $150 \mu \mathrm{m}$ for the PLLA-CD scaffolds and between 100 and $150 \mu \mathrm{m}$ for the PLLA-MED scaffolds. Both PLLA brands present the same tendency; the highest micropore dimension is obtained for the (5/95) in both series and then decreases with the polymer concentration, until disappearing for the (20/80) scaffolds fabricated with industrial PLLA, as observed on the SEM pictures. For the PLLA-CD scaffolds the micropore dimensions range from 8 to $0 \mu \mathrm{m}$ and for the PLLA-MED scaffolds from 9 to $2 \mu \mathrm{m}$. 


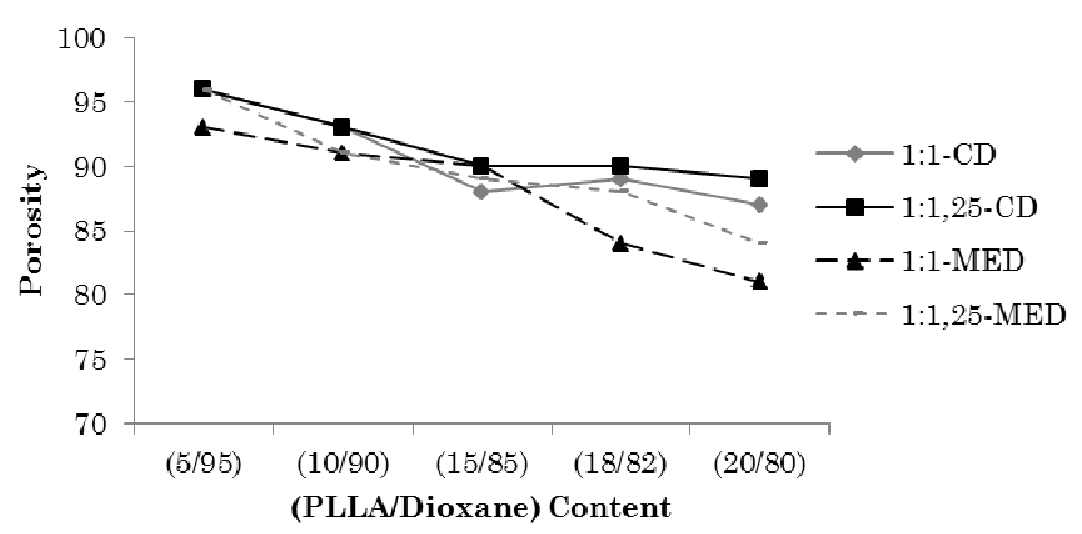

Figure 3-5: Porosity of (5/95), (10/90), (15/85), (18/82), (20/80) PLLA-CD and PLLA-MED scaffolds.

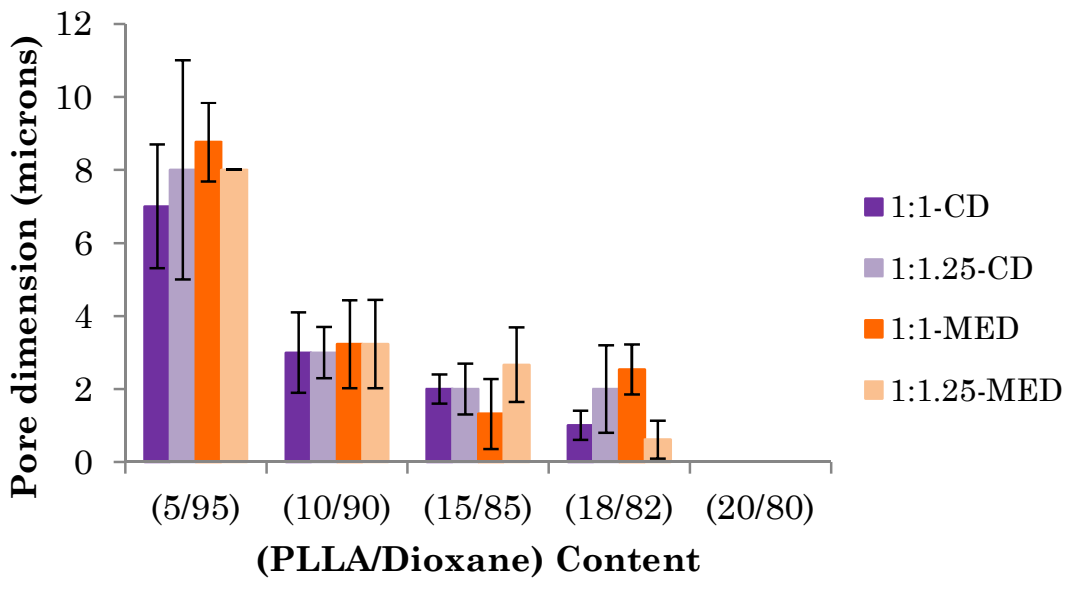

Figure 3-6: Micropore dimensions of PLLA-CD and PLLAMED scaffolds, values obtained by direct measurement from the SEM pictures of five pores of three different scaffolds. 


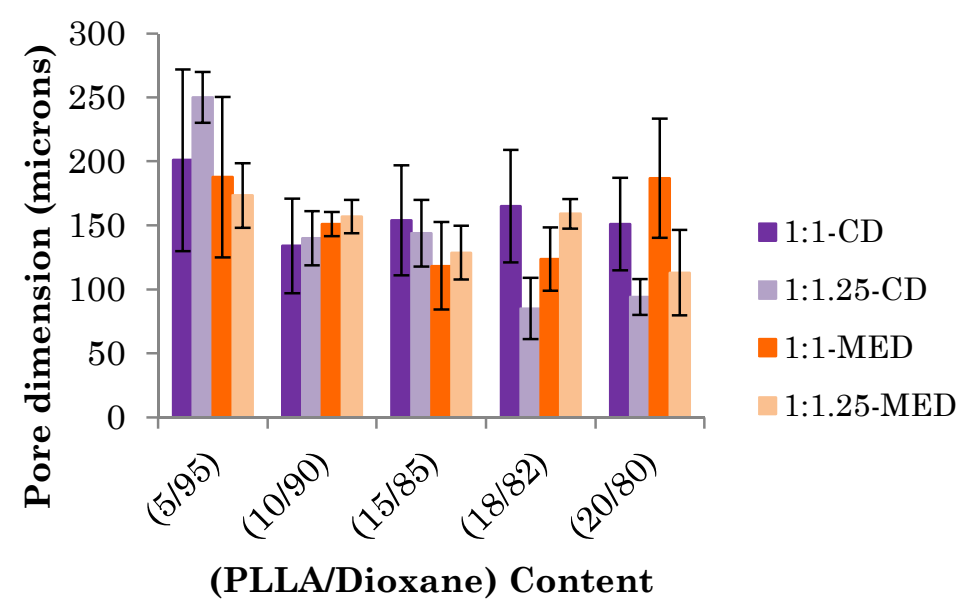

Figure 3-7: Macropore dimensions of PLLA-CD and PLLAMED scaffolds, values obtained by direct measurement from the SEM pictures of five pores of three different scaffolds.

\subsubsection{Mechanical properties}

The curves representative of the highest and the lowest elastic modulus for each type of PLLA and series are represented in Figure 3-8 and Figure 3-9, focusing on the elastic area marked by a dotted line. Figure 3-10 represents the compression curves of the 1:1-(5/95), (10/80), (15/85), (18/82), (20/80)-PLLAMED scaffolds in order to evaluate the mechanical resistance of the scaffolds with different compositions from the same series. This series is chosen to be represented because PLLAMED is the material selected for further degradation and implantation studies. The elastic modulus of the scaffolds can be calculated from the compression curves as explained in section 2.2 .4$, p.59. The compression curves of the (15/85) scaffolds of both PLLA are shown in Figure 3-11. Table 3-1 presents the apparent elastic modulus of each formulation of 
scaffolds. In order to visualize the tendency of the evolution of the elastic modulus as a function of the formulation a graph representing the elastic modulus is represented in Figure 3-12.

\section{$\underline{\text { PLLA-CD }}$}

The highest elastic modulus obtained for either series $1: 1 / 1: 1.25$ is for the scaffolds with $15 \%$ PLLA, corresponding to the $1: 1-(15 / 85)$ scaffold with a modulus of $8.9 \pm 0.8 \mathrm{MPa}$. The lowest measured elastic moduli are for the 1:1-(5/95) and 1:1.25-(5/95) scaffolds, $0.2 \pm 0.1 \mathrm{MPa}$ and $0.1 \pm 0.1 \mathrm{MPa}$, respectively. In general, the elastic moduli of the 1:1 series are bigger than those of the 1:1.25 series, with the exception of the (18/82) sample.

\section{$\underline{\text { PLLA-MED }}$}

The highest moduli are obtained for the 1:1-(18/82) and 1:1.25(15/85) scaffolds with $6.06 \pm 0.92 \mathrm{MPa}$ and $4.8 \pm 0.4 \mathrm{MPa}$, respectively. And the lowest correspond to those calculated for the (5/95) samples of both series.

The shape of the compression curves is similar for each type of PLLA. In Figure 3-8 and Figure 3-9, it is possible to observe the difference in the shape between the elastic zones in the compression curves, of the (5/95) and (15/85) scaffolds for each series and PLLA type. It can be appreciated how the elastic area is inexistent and the yield point is occurring instantly for the 1:1-(5/95) scaffold. It can also be seen that the yield point for a certain strain (dotted line) increases towards higher stress level when the polymer content increases. Therefore, the plateau area is reached at higher stress level when polymer content increases. 
In Figure 3-11 it can be observed that the yield point of the PLLA-MED scaffolds is achieved at higher stress and strain than for the PLLA-CD scaffolds.

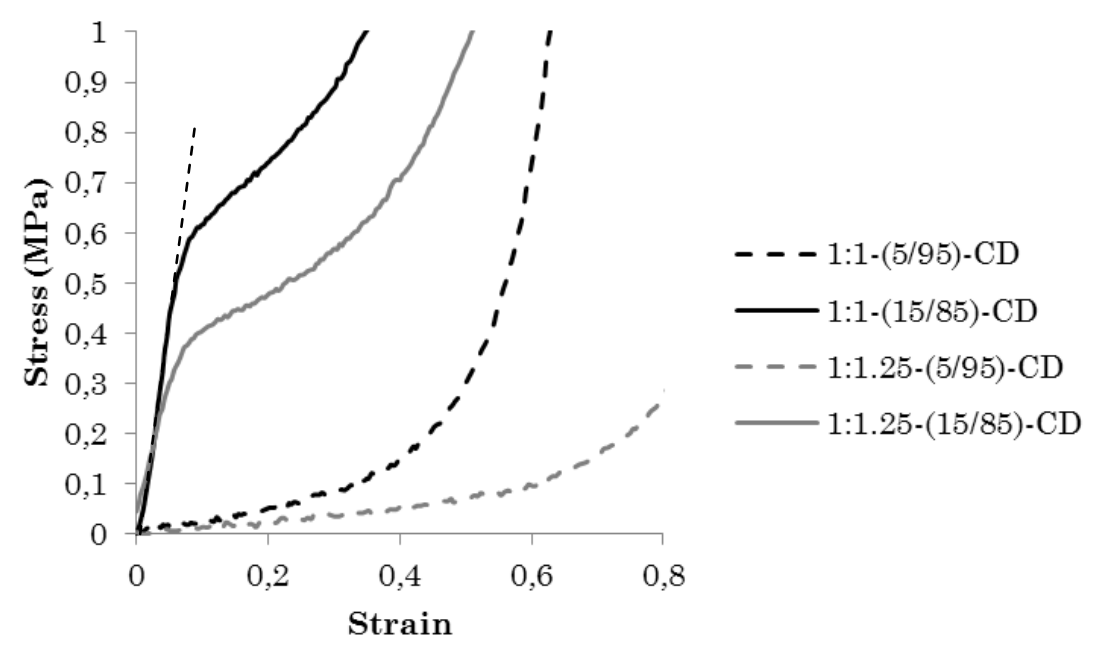

Figure 3-8: Compression test curves of 1:1/1:1.25(5/95)/(15/85)- CD scaffolds

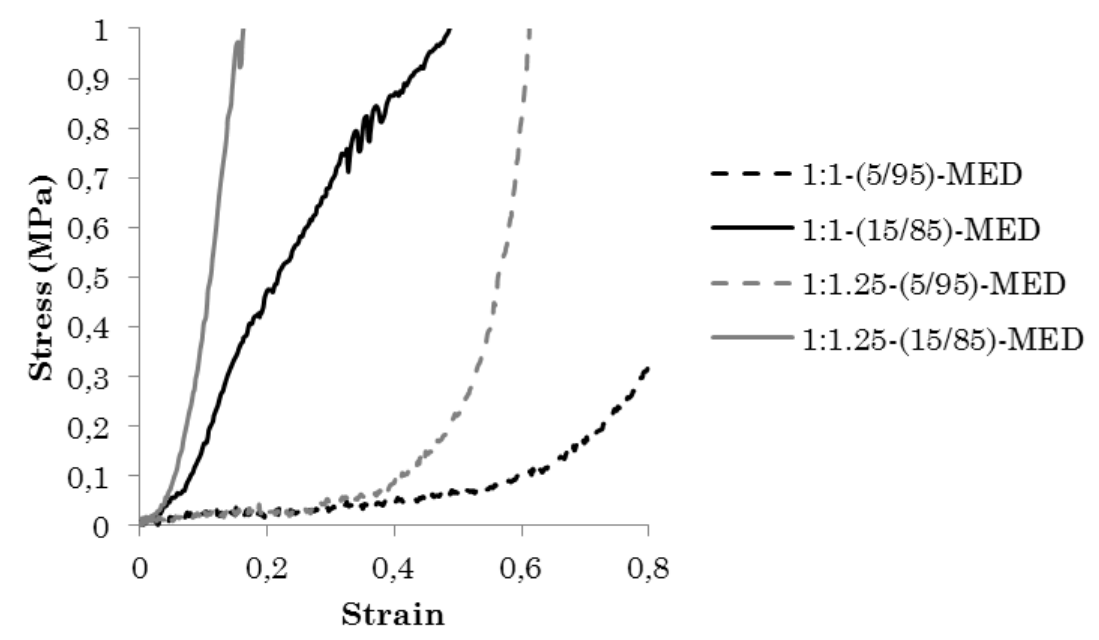

Figure 3-9: Compression test curves of 1:1/1:1.25-(5/95)/(15/85)MED scaffolds. 


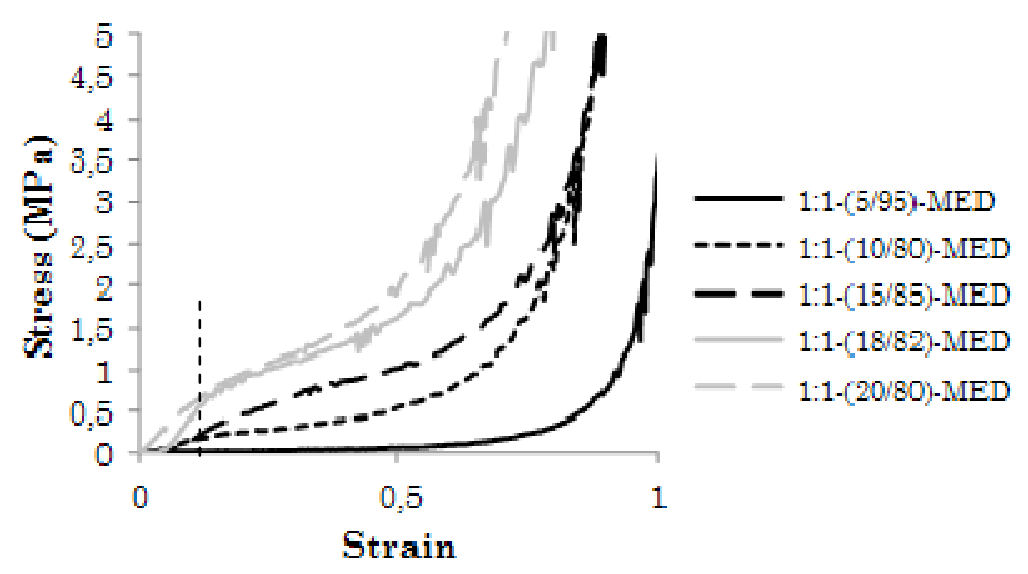

Figure 3-10: Compression test curves of 1:1/1:1.25$(5 / 95) /(10 / 80) /(15 / 85) /(18 / 82) /(20 / 80)-$ MED scaffolds.

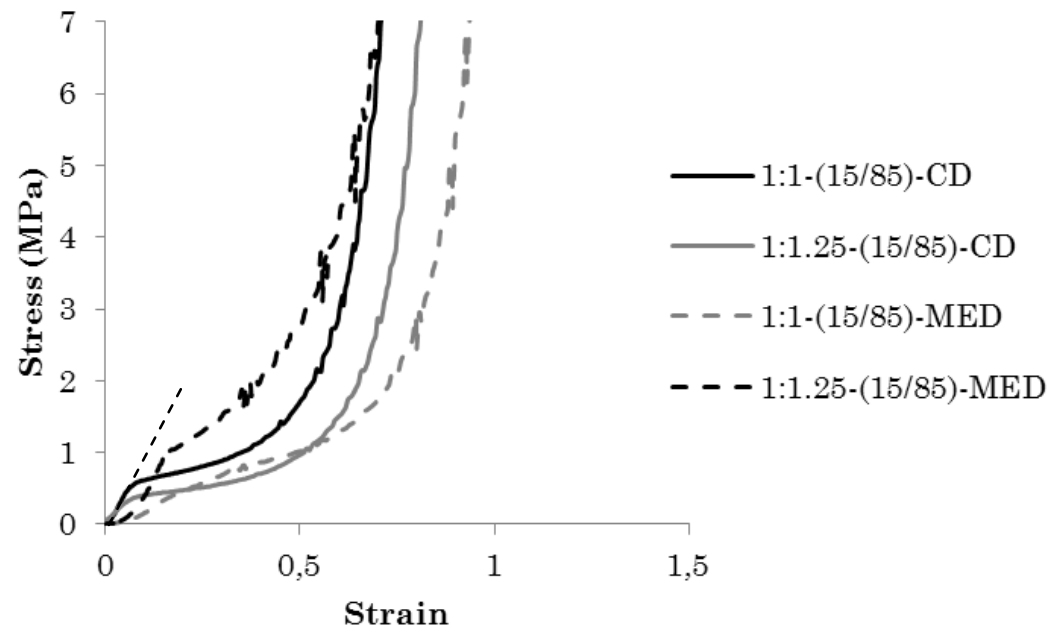

Figure 3-11: Compression test curves of 1:1/1:1.25-(15/85)MED/CD scaffolds. 
Table 3-1: Elastic modulus of the PLLA-CD and PLLA-MED scaffolds from the elastic zone of the compressive curves.

\begin{tabular}{lcccc}
\hline \multirow{2}{*}{$\begin{array}{l}\text { PLLA/dioxane) } \\
\text { content }\end{array}$} & \multicolumn{2}{c}{$\begin{array}{c}\text { E elastic (MPa) } \\
\text { PLLA-CD }\end{array}$} & \multicolumn{2}{c}{$\begin{array}{c}\text { E elastic (MPa) } \\
\text { PLLA-MED }\end{array}$} \\
\cline { 2 - 5 } & $\mathbf{1 : 1}$ & $\mathbf{1 : 1 . 2 5}$ & $\mathbf{1 : 1}$ & $\mathbf{1 : 1 . 2 5}$ \\
\hline $\mathbf{( 5 / 9 5 )}$ & $0.2 \pm 0.1$ & $0.1 \pm 0.1$ & $0.2 \pm 0.1$ & $0.1 \pm 0.03$ \\
$\mathbf{( 1 0 / 9 0 )}$ & $2.4 \pm 1.5$ & $1.7 \pm 1.2$ & $1.7 \pm 0.2$ & $0.6 \pm 0.01$ \\
$\mathbf{( 1 5 / 8 5 )}$ & $\mathbf{8 . 9} \pm \mathbf{0 . 8}$ & $5.3 \pm 2.7$ & $3.5 \pm 0.6$ & $4.8 \pm 0.4$ \\
$\mathbf{( 1 8 / 8 2 )}$ & $2.6 \pm 1.1$ & $3.4 \pm 1.0$ & $6.1 \pm 0.9$ & $2.4 \pm 0.4$ \\
$\mathbf{( 2 0 / 8 0 )}$ & $1.9 \pm 1.7$ & $1.8 \pm 1.3$ & $5.7 \pm 0.4$ & $2.3 \pm 0.5$ \\
\hline
\end{tabular}

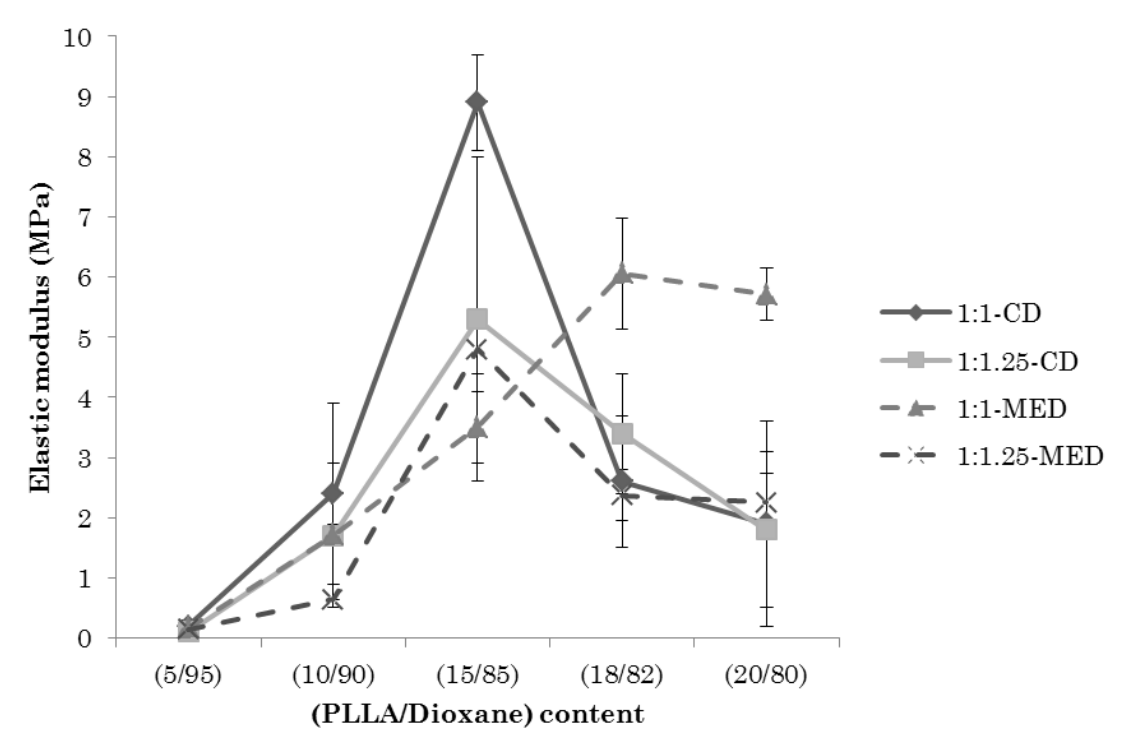

Figure 3-12: Apparent elastic compression modulus of the 1:1/1:1.25-(15/85)- PLLA-CD and PLLA-MED scaffolds 


\subsubsection{Calorimetric properties}

Scans of first and second heating experiments are shown in Figure 3-13 of the 1:1-(15/85) scaffolds of PLLA-MED and CD. Similar scans were obtained for the other compositions.

Table 3-2 presents the enthalpic glass transition temperature, the maximum of the temperature of crystallization and the maximum of the melting temperature of the (15/85) of both PLLA after the first and the second scan of DSC test. Table 3-3, Table 3-4, and Table 3-5 compile the enthalpic glass transition temperature of the first scan, the maximum of the melting temperature of the first scan and the crystallinity of the first and second scans, respectively, obtained from the DSC curves for each composition. In most of these tables the data were calculated only after the first scan in order to have information of the materials after their fabrication. The enthalpic glass transition and the maximum of the melting temperature were obtained for each curve by the method of integration of the heat flow curves. The enthalpic glass transition temperature was calculated and corresponds to the intersection of the enthalpic lines at the liquid and glass states in the enthalpy vs. temperature diagram.

The crystallinity $\left(\chi_{\mathrm{c}}(\%)\right)$ was determined by the equation (6):

$$
\chi_{c}(\%)=\frac{\Delta H m-\Delta H c}{\Delta H m_{100 \%}} \cdot 100
$$

where $\Delta H m$ and $\Delta H c$ are the enthalpy of melting and the enthalpy of crystallization in the case in which a crystallisation peak appears, respectively. $\Delta H_{100 \%}$ is the heat of melting of the $100 \%$ crystallised PLLA; a value of $93.1 \mathrm{~J} / \mathrm{g}$ (167) was used.

Figure 3-13 shows that any crystallisation peak appears after the first scan but only after the second one. In the case of the medical grade PLLA-MED two crystallization peaks at low 
temperature and another one at higher temperature can be observed in the second scan in the case of the PLLA-MED. Glass transition temperature occurs at lower temperature in the first scan than at the second one.

The glass transition temperature is around $60^{\circ} \mathrm{C}$ for all scaffolds (Table 3-3); there are no significant changes between the different PLLA used to fabricate the samples.

The maximum of the melting temperature (Table 3-4) is lower for the PLLA-CD scaffolds (around $164^{\circ} \mathrm{C}$ ) than for the PLLAMED (around $180^{\circ} \mathrm{C}$ ) scaffolds. For both brands there are no significant changes between the different polymer concentration and polymer/ porogen ratio. The crystallinity (Table 3-5) of the PLLA-CD scaffolds is lower (around 40\%) than that of the PLLA-MED scaffolds (around 50\%). For both brands there are no significant changes in the different polymer concentrations and polymer/ porogen ratios.

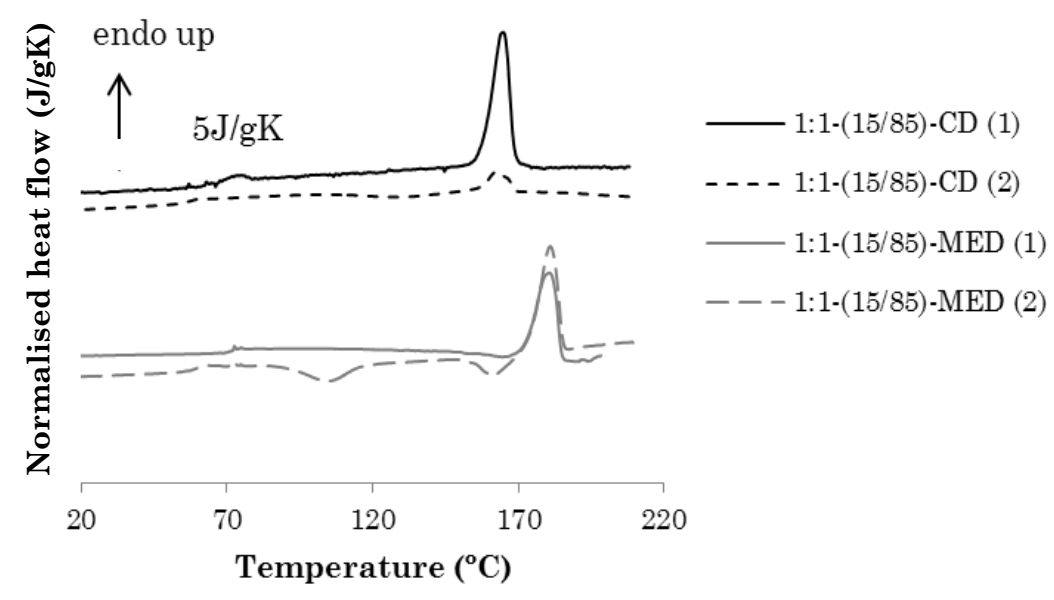

Figure 3-13: Normalised heat flow (J/gK) as a function of the temperature from the DSC first (1) and second scan (2) of 1:1(15/85) PLLA-CD and PLLA-MED scaffolds. 
Table 3-2: Enthalpic glass transition temperature (Tg), maximum of crystallization temperature (Tc) of the second peak in the case of PLLA-MED and melting temperature (Tm) after the first and second scans of 1:1-(15/85) PLLA-MED and PLLA-CD scaffolds.

\begin{tabular}{|c|c|c|c|c|c|c|}
\hline & CD & MED & CD & MED & CD & MED \\
\hline$N^{\circ}$ Scan & \multicolumn{2}{|c|}{$\operatorname{Tg}\left({ }^{\circ} \mathrm{C}\right)$} & \multicolumn{2}{|c|}{$\operatorname{Tc}\left({ }^{\circ} \mathrm{C}\right)$} & \multicolumn{2}{|c|}{$\operatorname{Tm}\left({ }^{\circ} \mathrm{C}\right)$} \\
\hline First & 63.0 & 65.3 & - & - & 164.3 & 180.6 \\
\hline Second & 56.5 & 59.0 & 130.3 & 103.3 & 161.2 & 180.9 \\
\hline
\end{tabular}

Table 3-3: Enthalpic glass transition temperature of PLLACD and PLLA-MED scaffolds.

\begin{tabular}{lcccc}
\hline \multirow{2}{*}{ Samples } & \multicolumn{2}{c}{${\mathbf{T g ~}\left({ }^{\circ} \mathbf{C}\right)}^{c}$} & \multicolumn{2}{c}{$\begin{array}{c}\text { Tg }\left({ }^{\circ} \mathbf{C}\right) \\
\text { PLLA-MED }\end{array}$} \\
\cline { 2 - 5 } & $\mathbf{1 : 1}$ & $\mathbf{1 : 1 . 2 5}$ & $\mathbf{1 : 1}$ & $\mathbf{1 : 1 . 2 5}$ \\
\hline$(5 / 95)$ & 63.7 & 58.3 & 69.0 & 64.4 \\
$(10 / 90)$ & 60.4 & 66.6 & 66.1 & 65.9 \\
$(15 / 85)$ & 63.0 & 60.3 & 65.3 & 72.8 \\
$(18 / 82)$ & 58.0 & 58.9 & 68.8 & 63.4 \\
$(20 / 80)$ & 62.7 & 64.7 & 68.6 & - \\
\hline
\end{tabular}

Table 3-4: Maximum of the melting temperature of PLLA-CD and PLLA-MED scaffolds.

\begin{tabular}{lcccc}
\hline \multirow{2}{*}{ Samples } & \multicolumn{2}{c}{ Tm $\left({ }^{\mathbf{o}} \mathbf{C}\right)$} & \multicolumn{2}{c}{ Tm $\left({ }^{\mathbf{0}} \mathbf{C}\right)$} \\
& $\mathbf{P L L A - C D}$ & \multicolumn{2}{c}{ PLLA-MED } \\
\cline { 2 - 5 } & $\mathbf{1 : 1}$ & $\mathbf{1 : 1 . 2 5}$ & $\mathbf{1 : 1}$ & $\mathbf{1 : 1 . 2 5}$ \\
\hline $\mathbf{( 5 / 9 5 )}$ & 163.8 & 163.6 & 181.3 & 180.4 \\
\hline$(10 / 90)$ & 163.3 & 163.9 & 179.4 & 179.4 \\
\hline$(15 / 85)$ & 164.3 & 163.6 & 180.6 & 181.9 \\
\hline$(18 / 82)$ & 164.8 & 164.9 & 180.8 & 180.6 \\
\hline$(20 / 80)$ & 164.1 & 163.4 & 181.9 & - \\
\hline
\end{tabular}


Table 3-5: Crystallinity of PLLA-CD and PLLA-MED scaffolds after the first scan.

\begin{tabular}{lcccc}
\hline \multirow{2}{*}{ Samples } & \multicolumn{2}{c}{$\begin{array}{c}\boldsymbol{\chi}_{\mathbf{c}}(\mathbf{\%}) \\
\text { PLLA-CD }\end{array}$} & \multicolumn{2}{c}{$\begin{array}{c}\boldsymbol{\chi} \mathbf{c}(\%) \\
\text { PLLA-MED }\end{array}$} \\
\cline { 2 - 5 } & $\mathbf{1 : 1}$ & $\mathbf{1 : 1 . 2 5}$ & $\mathbf{1 : 1}$ & $\mathbf{1 : 1 . 2 5}$ \\
\hline$(5 / 95)$ & 40 & 40 & 51 & 57 \\
\hline$(10 / 90)$ & 36 & 37 & 52 & 51 \\
\hline$(15 / 85)$ & 38 & 38 & 51 & 56 \\
\hline$(18 / 82)$ & 44 & 38 & 51 & 52 \\
\hline$(20 / 80)$ & 40 & 38 & 54 & - \\
\hline
\end{tabular}





\subsection{DEGRADATION EXPERIMENTS}

PLLA, due to its ester bond, can degrade in the human body, the degradation process leads to the loss of mechanical and physical-chemical properties. One way to determine the degradation process of PLLA scaffolds is by simulation through in vitro incubation in phosphate bufferer saline solution (PBS) at $37^{\circ} \mathrm{C}$ in order to recreate the conditions of $\mathrm{pH}$ and osmolarity of the human body. In this section, the effect of degradation time on the properties of the scaffolds is presented. Two types of degradation experiments were performed; first a static degradation experiment lasting 6, 12, 18,24 weeks and 1 year, which enables the evaluation of the effect of the hydrolytic degradation on the PLLA scaffolds. To maximise the simulation of degradation of PLLA in the human body, in such parts as joints, it is necessary to pay attention to the mechanical solicitation which can affect the degradation process. For this aim a second degradation experiment was performed; a fatigue assay, under a compressive dynamic loading in PBS at $37^{\circ} \mathrm{C}$, was applied during 1,2, 4 and 6 weeks on the scaffolds. These in vitro degradation experiments were performed on the scaffold formulation (1:1)-(15/85)-PLLAMED selected for the fabrication of highly porous scaffolds in section 4.1.2, p.176. 


\subsubsection{Static degradation}

\subsubsection{Morphology}

As can be seen in the SEM micrographs in Figure 3-14, the macro and microstructure of the different scaffolds submitted to different periods of degradation do not present significant changes up to 24 weeks. The trabeculae remain intact and the macropores are still highly interconnected. Table 3-6 shows the average macropores size determined by microtomography. The microtomography was carried out by the Group of Structural Mechanics and Materials Modelling, of the University of Zaragoza, in Spain, to determine the average macropores size.

The pore dimension increases with degradation time as it can be demonstrated in Table 3-6. But it was not really possible to appreciate a change on the micropore dimension even after 1 year of incubation in PBS. However a significant change was observed after 1 year when most of the trabeculae appeared broken in the SEM pictures. When, after 1 year, the scaffolds are manipulated they disaggregate easily. 


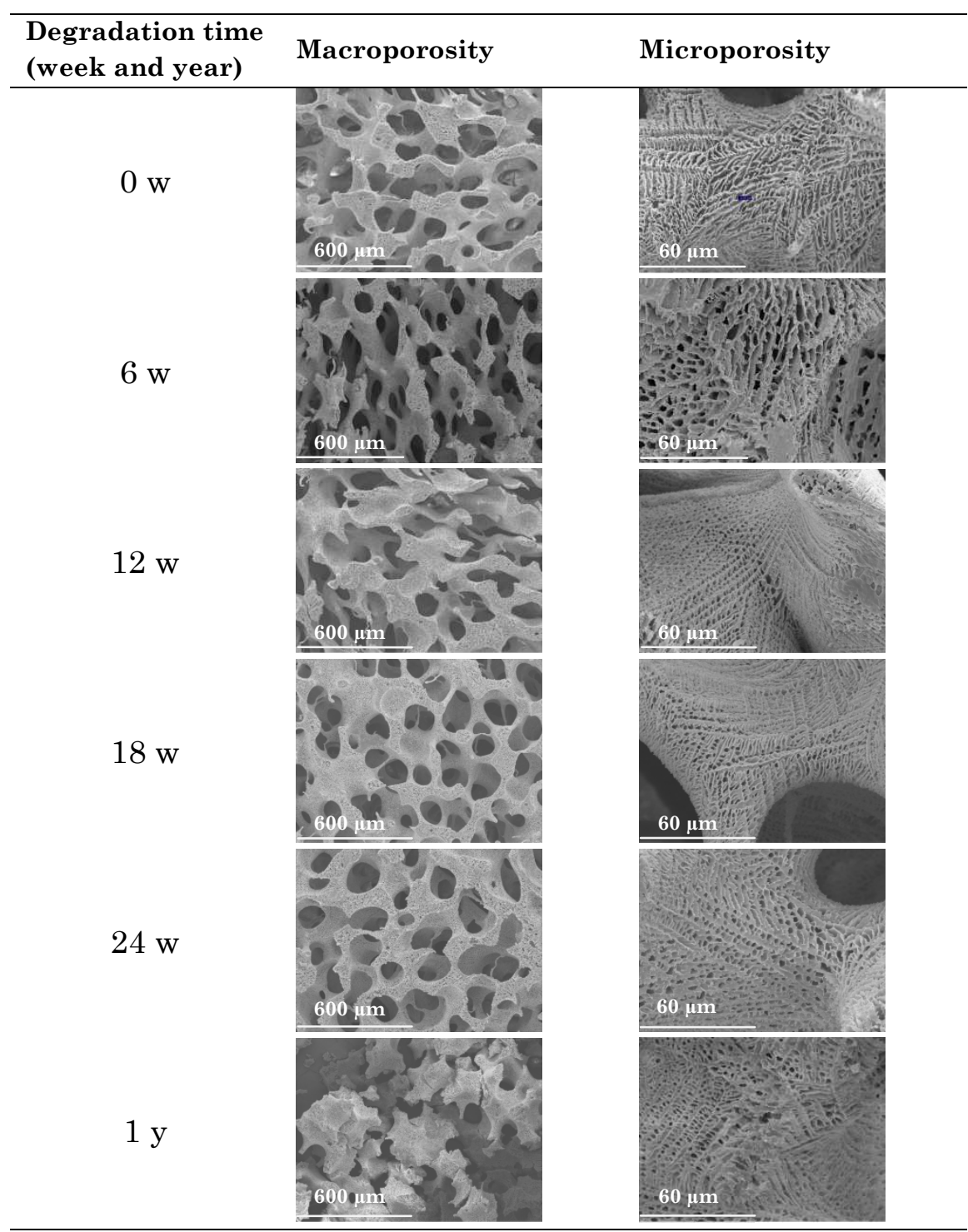

Figure 3-14: SEM pictures at different magnifications and degradation times of the section of the (1:1)-(15/85)-PLLAMED scaffold showing the effect of static degradation in PBS on scaffolds morphology. 


\subsubsection{Mass evolution}

The evolution of the mass of the samples as a function of the degradation time was determined. Figure 3-15 shows how the mass loss of the different scaffolds changed with time of degradation. The mass loss of the samples was evaluated using the following equation 7 :

$$
\text { mass remaining }(\%)=\frac{m_{i}-m_{f}}{m_{i}} \cdot 100
$$

where $m_{i}$ is the initial mass and $m_{f}$ the final mass of the samples.

Contrary to what was expected it seems that the scaffolds mass loss is hardly observed. Furthermore it can be observed that the mass residue increase after 6 and 18 weeks of degradation. This can be due to the precipitation of some salts on the surface of the materials as observed in the dynamic degradation. Elemental analysis was performed after the degradation tests as observed in Figure 3-31 in the fatigue essay. 


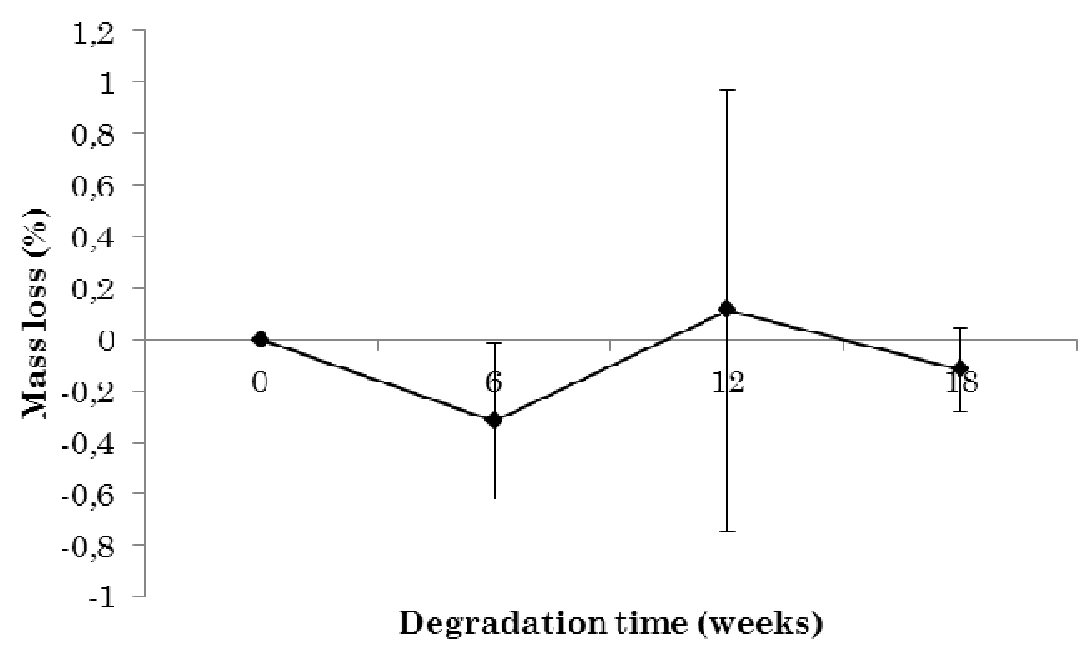

Figure 3-15: Percentage of mass remaining after $0,6,12$, and 18 weeks of static degradation for the 1:1-15\%-MED scaffold.

\subsubsection{Mechanical properties}

The influence of the static degradation experiment on the mechanical properties of PLLA scaffolds was evaluated by compression test from which the elastic modulus was calculated. The compression test was performed by the Group of Structural Mechanics and Materials Modelling, of the University of Zaragoza, in Spain. The protocol used for this study is detailed in section 2.2.4, p.59 . The stress-strain curves obtained from the compression test are represented in Figure 3-16 and the elastic moduli are compiled in Figure 3-17 and Table 3-6. The curves show the three specific zones characteristic of a porous material. The plateau area, representing the collapse of the pores, is affected by degradation time. The slope of the pore collapse zone decreases with degradation time, and at 24 weeks was almost flat. Consequently the yield point decreased when the scaffolds 
degraded. The stress and strain curves show that pores started to collapse at lower stresses when the degradation time increased and this effect was particularly marked after 18 and 24 weeks. When degradation time increases the scaffolds hold the load less and less, so that less stress is required to deform more. The elastic moduli present a slight decrease after 12 weeks of degradation from 3.4 to $2.9 \mathrm{MPa}$ (Table 3-6). 


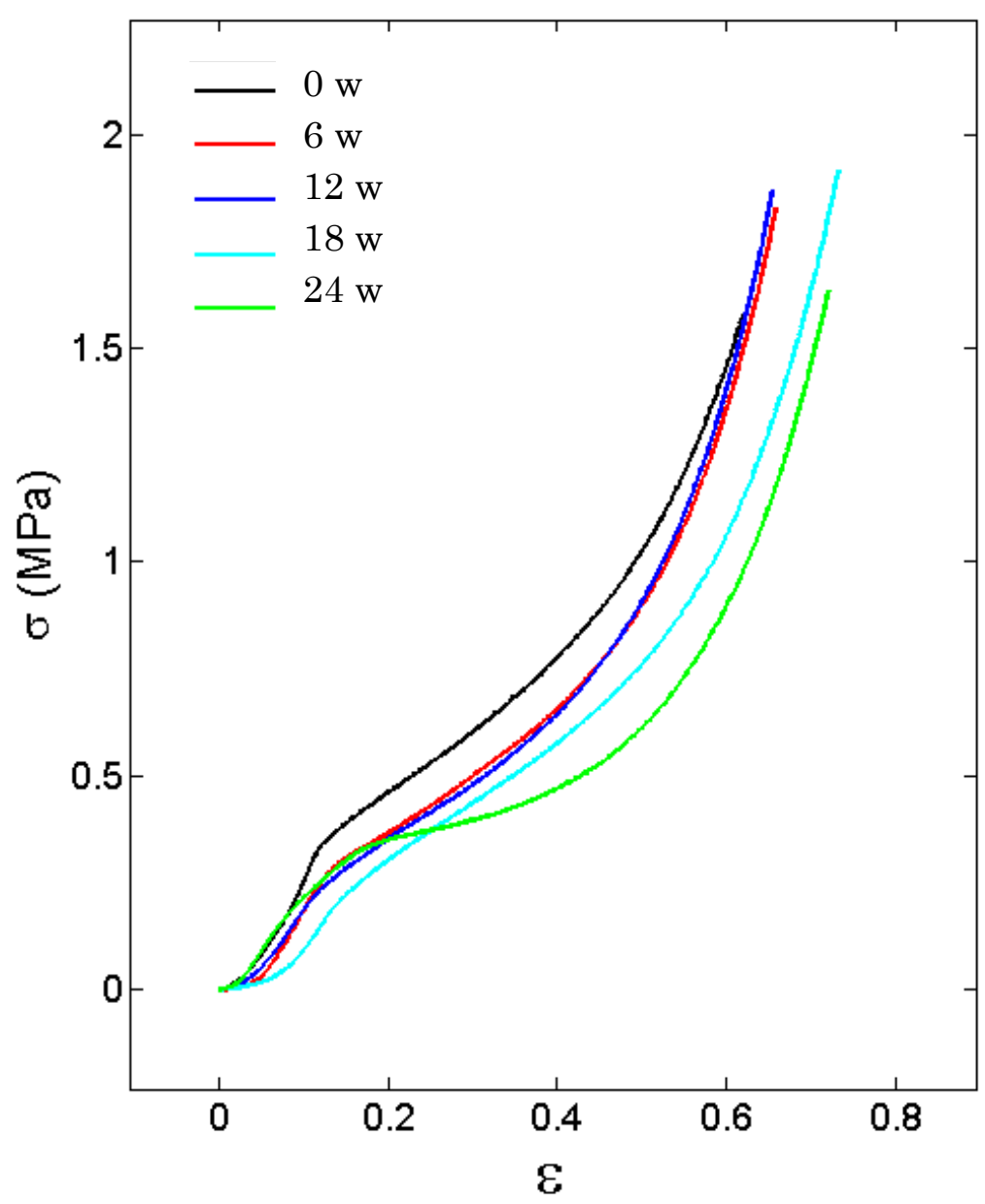

Figure 3-16: Stress - Strain curves of the compression test after $0,6,12,18$ and 24 weeks of static degradation for the 1:1-15\%-MED scaffold. 


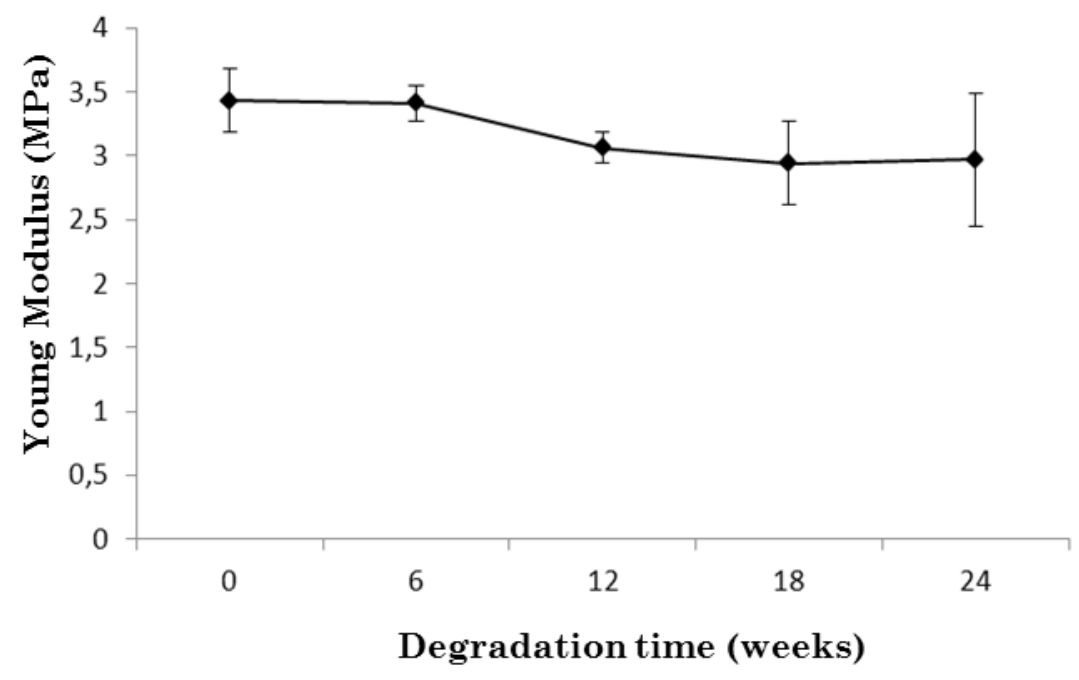

Figure 3-17: Apparent Young's modulus of the PLLA-1:1-15\%MED scaffold as a function of the degradation time under static in vitro conditions.

Table 3-6: Evolution of the apparent Young's modulus, permeability and macropore dimension as a function of the degradation time for the 1:1-15\%-MED scaffold under static conditions.

\begin{tabular}{lccc}
\hline & $\begin{array}{l}\text { Apparent } \\
\text { Young's } \\
\text { modulus } \\
\mathbf{( M P a )}\end{array}$ & $\begin{array}{l}\text { Permeability } \\
\mathbf{( m}^{\mathbf{2})}\end{array}$ & $\begin{array}{l}\text { Macropore } \\
\text { dimension } \\
(\boldsymbol{\mu m})\end{array}$ \\
\hline $\mathbf{0} \mathbf{~ w}$ & $3.43 \pm 0.25$ & $1.2 .10^{-7} \pm 0.1 .10^{-7}$ & $88 \pm 7$ \\
$\mathbf{6} \mathbf{~ w}$ & $3.41 \pm 0.14$ & $1.2 .10^{-7} \pm 0.1 .10^{-7}$ & $179 \pm 3$ \\
$\mathbf{1 2} \mathbf{~ w}$ & $3.06 \pm 0.12$ & $1.7 .10^{-7} \pm 0.3 \cdot 10^{-7}$ & $184 \pm 10$ \\
$\mathbf{1 8} \mathbf{~ w}$ & $2.94 \pm 0.33$ & $3.10 .10^{-7} \pm 0.3 .10^{-7}$ & $206 \pm 8$ \\
$\mathbf{2 4} \mathbf{~ w}$ & $2.97 \pm 0.52$ & $5.8 .10^{-7} \pm 1.4 .10^{-7}$ & $232 \pm 8$ \\
\hline
\end{tabular}




\subsubsection{Permeability}

Permeability tests allowed evaluation the effect of the degradation time on the water flow rate through the porous structures of the scaffolds. The permeability test was done by the Group of Structural Mechanics and Materials Modelling, of the University of Zaragoza, in Spain. The permeability test was performed accordingly to the Darcy's law (8) and valid for Reynolds numbers lower than 8.6

$$
k=\frac{\mu \cdot t \cdot Q}{A \cdot \Delta p}
$$

Where $k$ is the intrinsic permeability, $t$ the specimen thickness, $A$ the cross-sectional area, $Q$ is the volumetric flow rate, $\Delta p$ is the pressure difference across the sample and $\mu$ the dynamic fluid viscosity (deionized water at $10^{-3} \mathrm{~Pa}$ ). All the samples were immersed in PBS solution during 48 hours before testing. According to the experimental protocol, the fluid flow through the scaffold was increased at different rates $(20,40,60 \mathrm{ml} / \mathrm{min})$ and for each case the pressure difference between the exit and entrance to the scaffold was measured. The obtained results were averaged and the intrinsic permeability of the material was determined. Five replicates of each sample were measured for the sake of precision. The results of the permeability test are shown in Figure 3-18 and Table 3-6. It can be clearly observed that the permeability increased exponentially when the scaffolds were more and more degraded. 


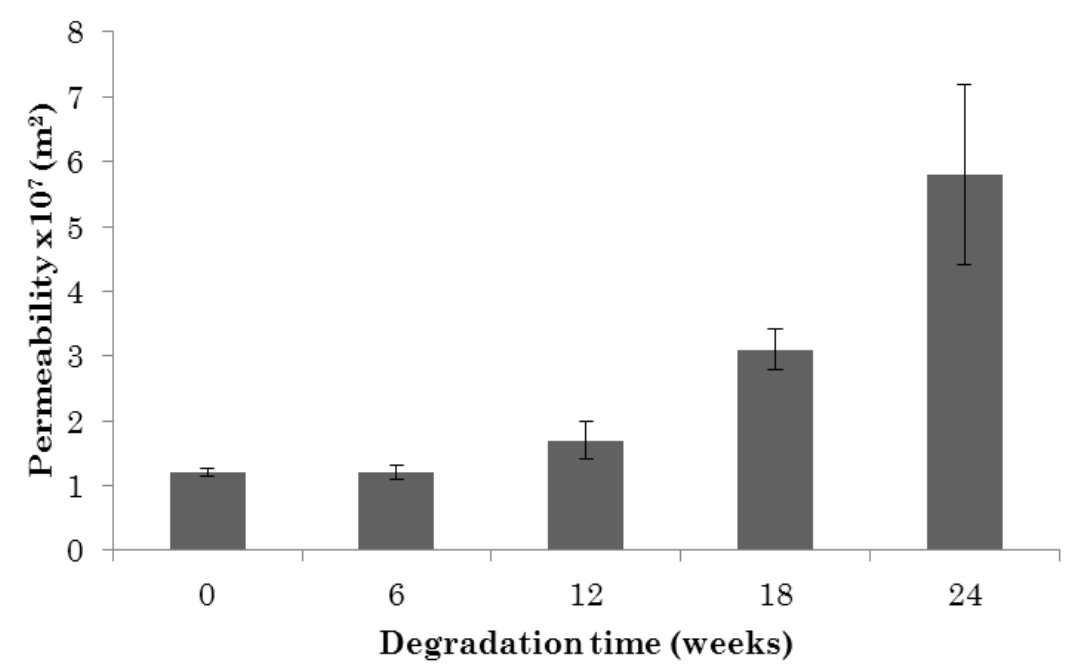

Figure 3-18: Evolution of the permeability of PLLA-1:1-15\%MED scaffold as a function of static degradation time in PBS.

\subsubsection{Calorimetric properties}

The influence of the static degradation process on the calorimetric properties of PLLA-1:1-15\%-MED scaffold were evaluated using DSC tests. The samples corresponding to different time of degradation $(0,6,12,18,24$ weeks and 1 year) were submitted to three temperature scans: two from $0^{\circ} \mathrm{C}$ to $210^{\circ} \mathrm{C}$ at $10^{\circ} \mathrm{C} / \mathrm{min}$ and the cooling between them from $210^{\circ} \mathrm{C}$ to $0^{\circ} \mathrm{C}$ at $10^{\circ} \mathrm{C} / \mathrm{min}$. The results from the DSC test are represented in Figure 3-19 and Figure 3-20. The curves of the first scan (1) present two differences between the nondegraded scaffolds and the degraded scaffolds. The degraded scaffolds present a double peak typical of double melt behaviour. An exception has to been made for the scaffolds degraded for 1 year. The double melting behaviour of PLLA is explained by the melting of originate crystals and the second peak by the melting of recrystallized crystals that can be caused by the degradation process. The cooling peaks shift 
towards a lower temperature as the scaffolds degrade. The second heat scan presents differences from the first one; two crystallization peaks appear, one with a maxima at low temperature (around $100^{\circ} \mathrm{C}$ ), and a smaller one with a maxima of around $160^{\circ} \mathrm{C}$. The second peak is explained to be the recrystallization process (137). The trend is the same for all of the scaffolds tested. The double melting peak does not appear at the second heating. The maximum of the melting process appears at a lower temperature in the second heating.

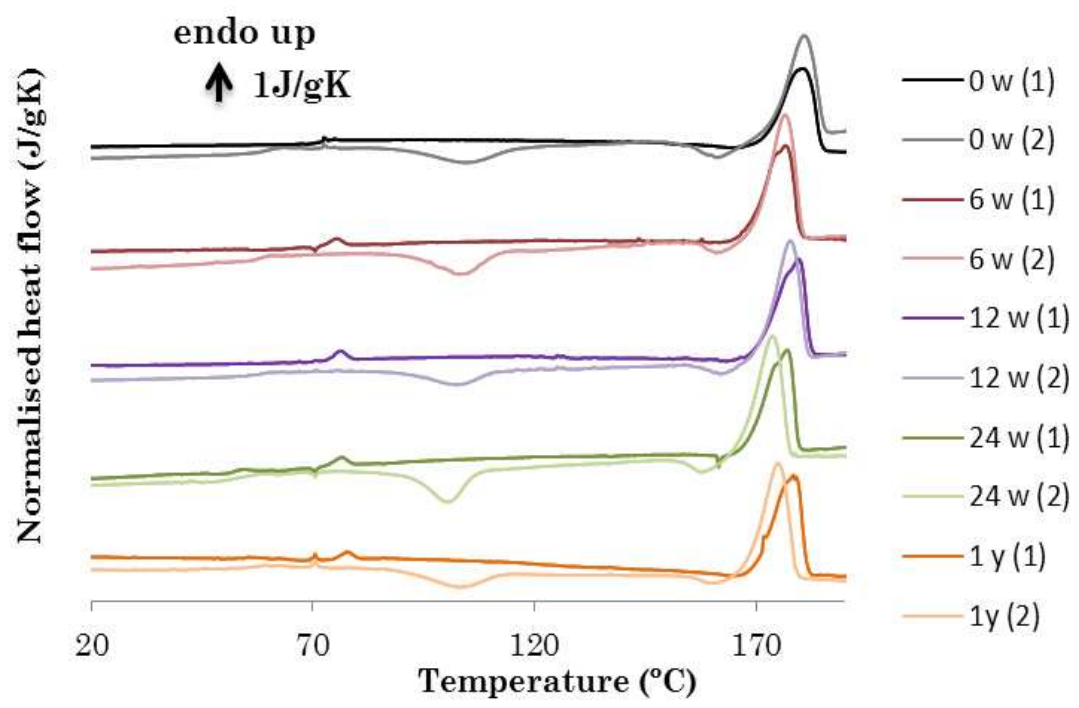

Figure 3-19: Normalised heat flow of the DSC first (1) and second (2) heating scans after $0,6,12,24$ weeks and 1 year of static degradation of PLLA-1:1-15\%-MED scaffolds. The heat flow was normalised by the mass of the samples and the heating rate at $\left(10^{\circ} \mathrm{C} / \mathrm{min}\right)$. The arrow mark represents a unit of normalised heat flow in $\mathrm{J} / \mathrm{gK}$. 


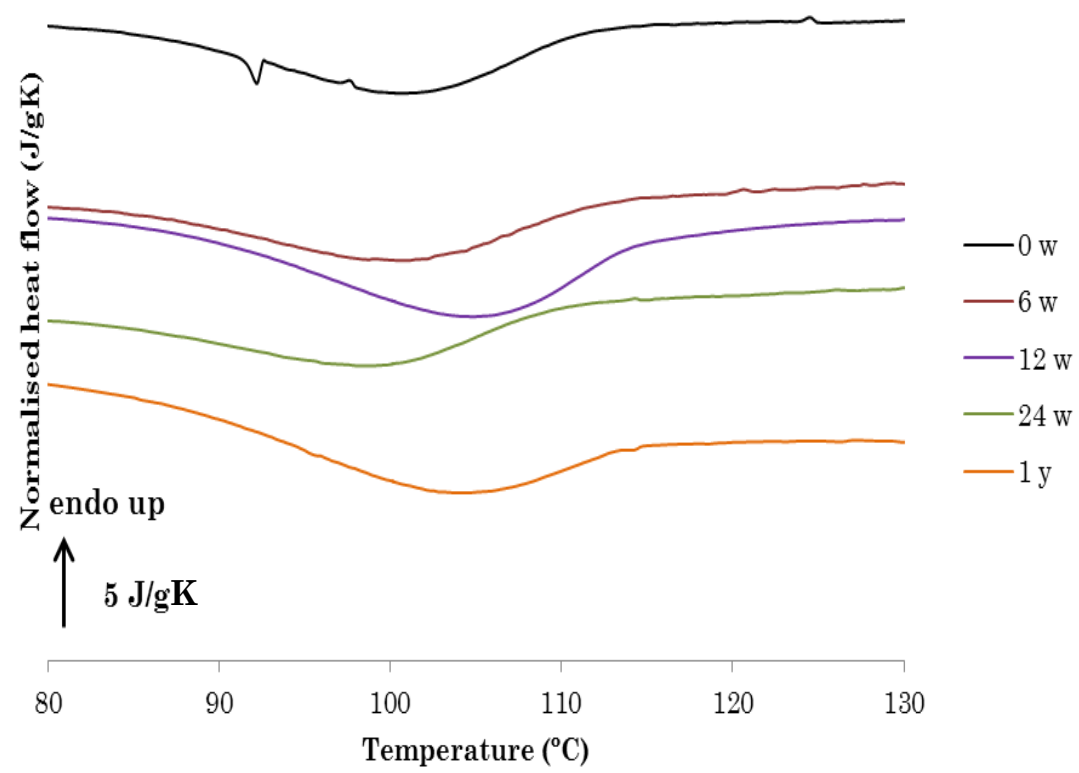

Figure 3-20: Normalised heat flow of the DSC cool scans after $0,6,12,24$ weeks and 1 year of static degradation of PLLA1:1-15\%-MED scaffolds. The heat flow was normalised by the mass of the samples and the heating rate at $10^{\circ} \mathrm{C} / \mathrm{min}$. The arrow mark represents 5 units of normalised heat flow in J/gK.

From these curves the enthalpic glass transition temperature (determined as the intersection temperature of the enthalpic lines at the liquid and glass states in the enthalpy vs. temperature diagram) and the crystallinity (see equation (1) in section 3.1.4, p.78) are compiled in Figure 3-21, Figure 3-23 and Table 3-7. Crystallinity measured after the first heating scan (1) is representative of the state of the sample after degradation and the one measured from the second heating scan (2) is representative of the ability of crystallization from the melt of the samples, previously degraded and melted. It 
can be observed that the glass transition temperature $\left(\mathrm{Tg}_{2}\right)$ and the crystallinity $\left(\chi \mathrm{c}_{2}\right)$ of the scaffolds at the second heating are smaller than those corresponding to the first one, for all the degradation times. The glass transition temperature at day 0 shifted from $72^{\circ} \mathrm{C}$ in the first scan to $58^{\circ} \mathrm{C}$ in the second. The crystallinity of the scaffolds after the different degradation times remained almost constant around $50 \%$, for the first heating. In the second heating scan it was smaller than in the first and increased with the time of degradation; the crystallinity is $13 \%$ for the non-degraded sample and increased up to $38 \%$ for the 1 year degraded sample.

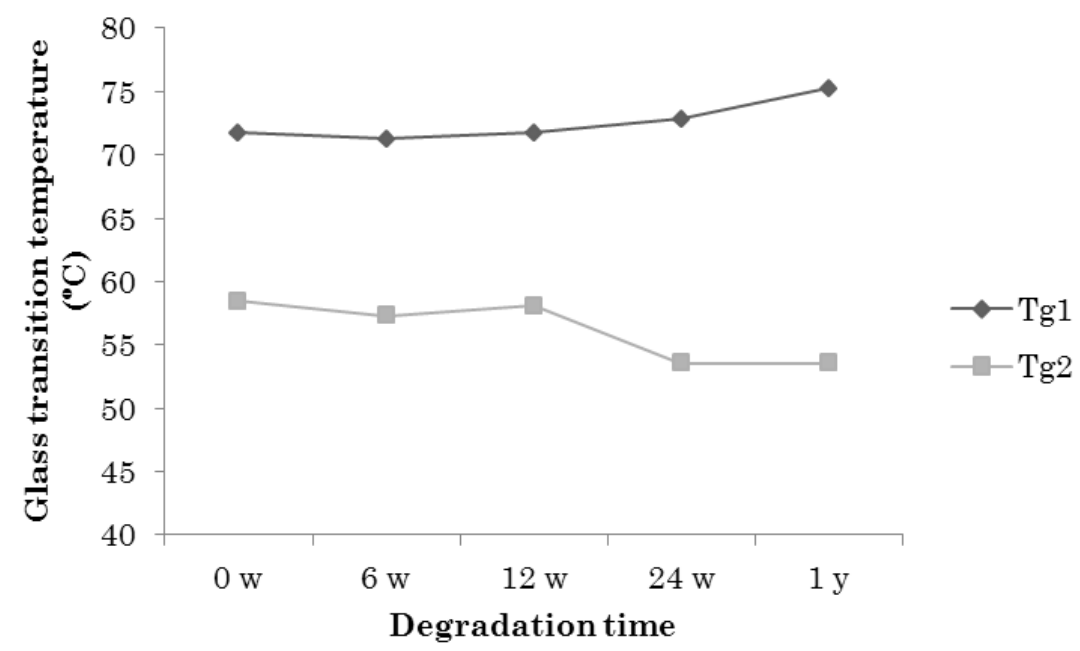

Figure 3-21: Glass transition temperature after the first $\left(\mathrm{Tg}_{1}\right)$ and second ( $\left.\mathrm{Tg}_{2}\right)$ DSC scan as a function of static degradation in $\mathrm{PBS}$ at $37^{\circ} \mathrm{C}$ after $0,6,12,24$ weeks and 1 year of PLLA-1:1-15\%-MED scaffolds. 


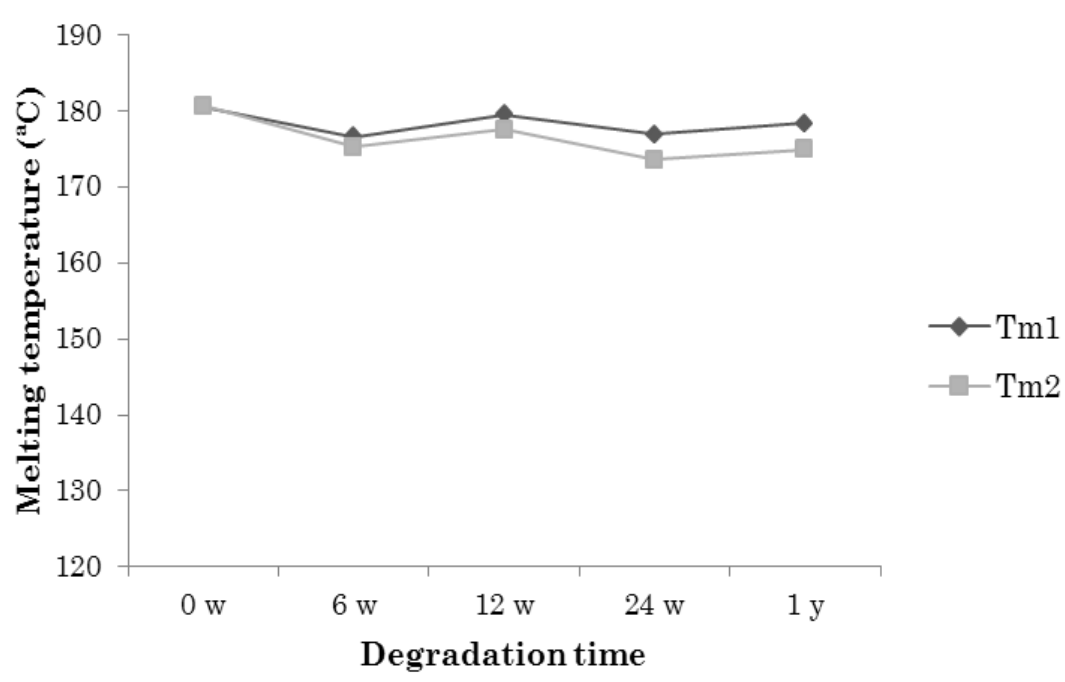

Figure 3-22: Melting temperature after the first $\left(\operatorname{Tm}_{1}\right)$ and second $\left(\mathrm{Tm}_{2}\right)$ DSC scan as a function of static degradation in PBS at $37^{\circ} \mathrm{C}$ after $0,6,12,24$ weeks and 1 year of PLLA-1:1$15 \%$-MED scaffolds.

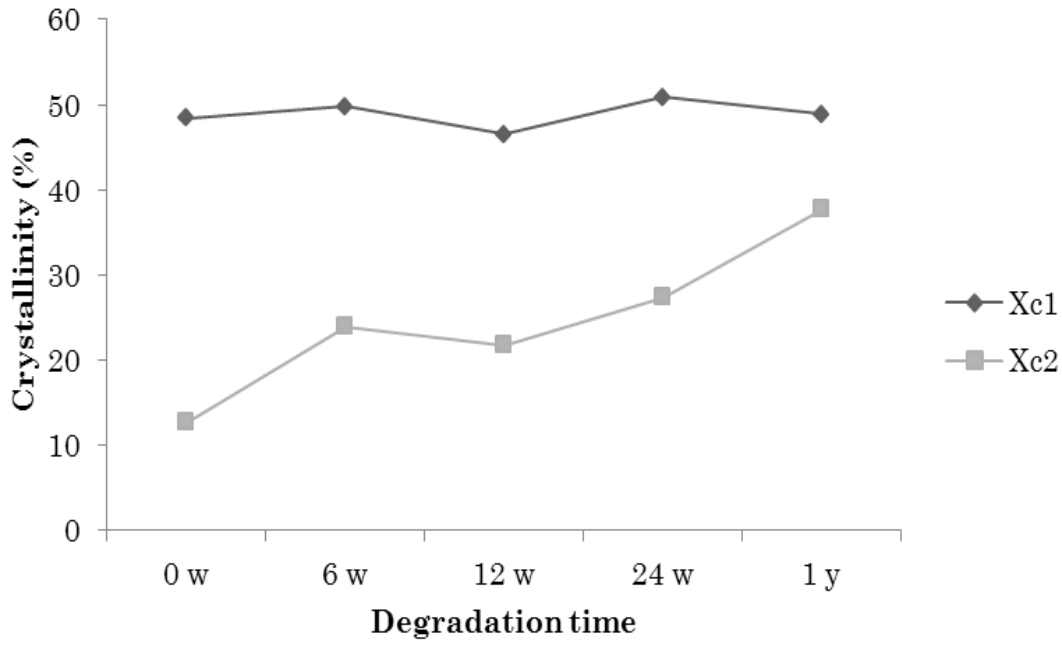

Figure 3-23: Crystallinity after the first $\left(\chi \mathrm{c}_{1}\right)$ and second $\left(\chi \mathrm{c}_{2}\right)$ DSC scan as a function of static degradation in PBS at $37^{\circ} \mathrm{C}$ after 0, 6, 12, 24 weeks and 1 year of PLLA-1:1-15\%-MED scaffolds. 
Table 3-7: Crystallinity $\left(\chi_{c}\right)$ and glass transition temperature (Tg) after the first (1) and second (2) DSC scan of the scaffolds after different time of static degradation in PBS at $37^{\circ} \mathrm{C}$.

\begin{tabular}{|c|c|c|c|c|}
\hline \multirow[t]{2}{*}{$\begin{array}{c}\text { Degradation } \\
\text { time }\end{array}$} & \multicolumn{2}{|c|}{ Crystallinity (\%) } & \multicolumn{2}{|c|}{$\begin{array}{l}\text { Glass transition } \\
\text { Temperature }\left({ }^{\circ} \mathrm{C}\right)\end{array}$} \\
\hline & $\chi_{c 1}$ & $\chi_{\mathrm{c} 2}$ & $\mathrm{Tg}_{1}$ & $\mathrm{Tg}_{2}$ \\
\hline $0 \mathrm{w}$ & 48 & 13 & 72 & 58 \\
\hline $6 \mathrm{w}$ & 50 & 24 & 71 & 57 \\
\hline $12 \mathrm{w}$ & 46 & 22 & 72 & 58 \\
\hline $24 w$ & 51 & 27 & 73 & 54 \\
\hline $1 \mathbf{y}$ & 49 & 38 & 75 & 54 \\
\hline
\end{tabular}

\subsubsection{Gel permeation chromatography}

From the GPC curves (Figure 3-24), it is possible to know the fraction (\%) of polymer chains length as a function of $\log (\mathrm{M})$. Consequently it is possible to evaluate the effect of degradation of the PLLA scaffolds on the molar mass distribution function. The weight average molar mass $(\mathrm{Mw})$ is represented in Figure 3-25, and the polydispersity index Mw/Mn in Figure 3-26, Mn being the number average molar mass. The distribution of the molar masses of the PLLA samples is described in Figure 3-24. Initially the non-degraded PLLA scaffold presented a narrow molecular mass distribution with weight average molar mass of almost $200 \mathrm{kDa}$. With time a higher fraction of short chains appear and average molar mass decreases (Figure 3-25). However, the GPC curve shows that a major fraction of chains keeps its molecular weight as the principal peak (Figure 3-24) does not change of position but lowers its intensity. The polydispersity index increases as the GPC curve shows that fraction of lower molecular weight chains increases (Figure 3-26). This evolution is characteristic 
of polymers that degrade in surface (from outside to the interior of the material). The longer the degradation time the more the peak shifts towards a lower molecular weight as the entire volume of the material becomes affected by the degradation process, especially for one year.

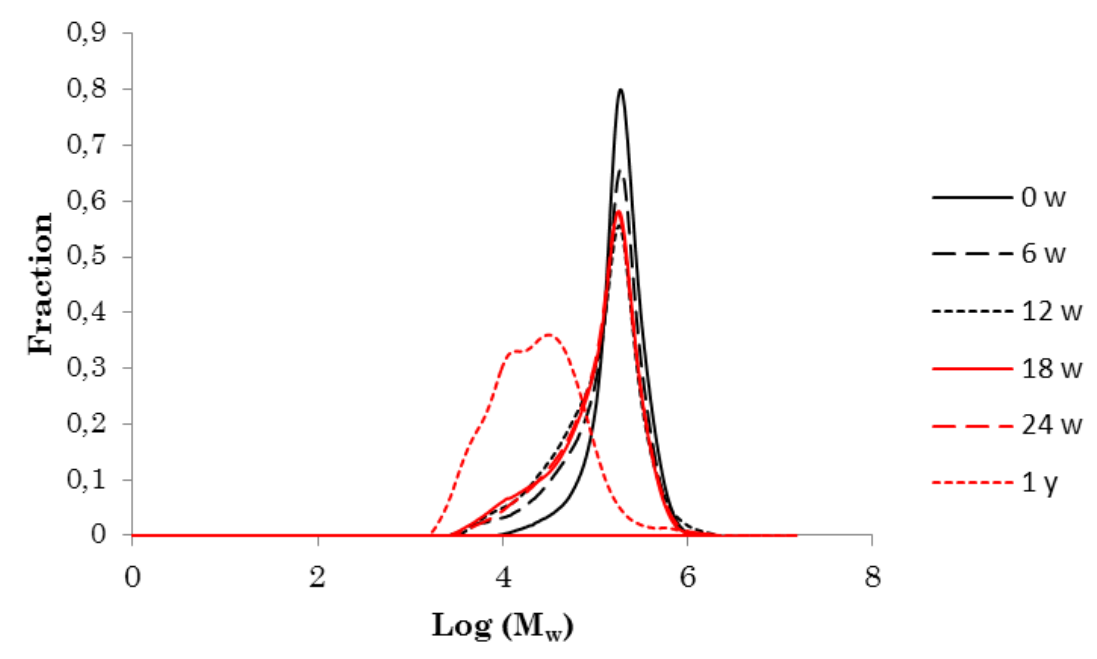

Figure 3-24: Gel permeation chromatography curves representing the fraction in percentage as a function of $\log \left(M_{\text {PLLA }}\right)$ of PLLA-1:1-15\%-MED scaffolds after 0, 6, 12, 18, 24 weeks and 1 year of static degradation. 


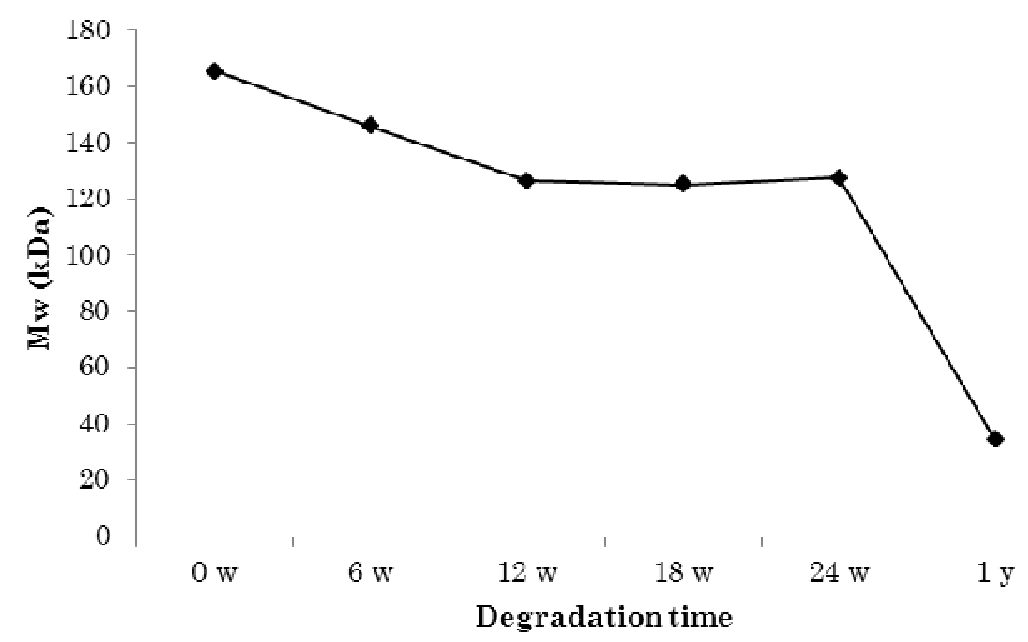

Figure 3-25: Evolution of the weight average molecular weight (Mw) as a function of degradation time of PLLA-1:1$15 \%-M E D$ scaffolds after $0,6,12,18,24$ weeks and 1 year of static degradation.

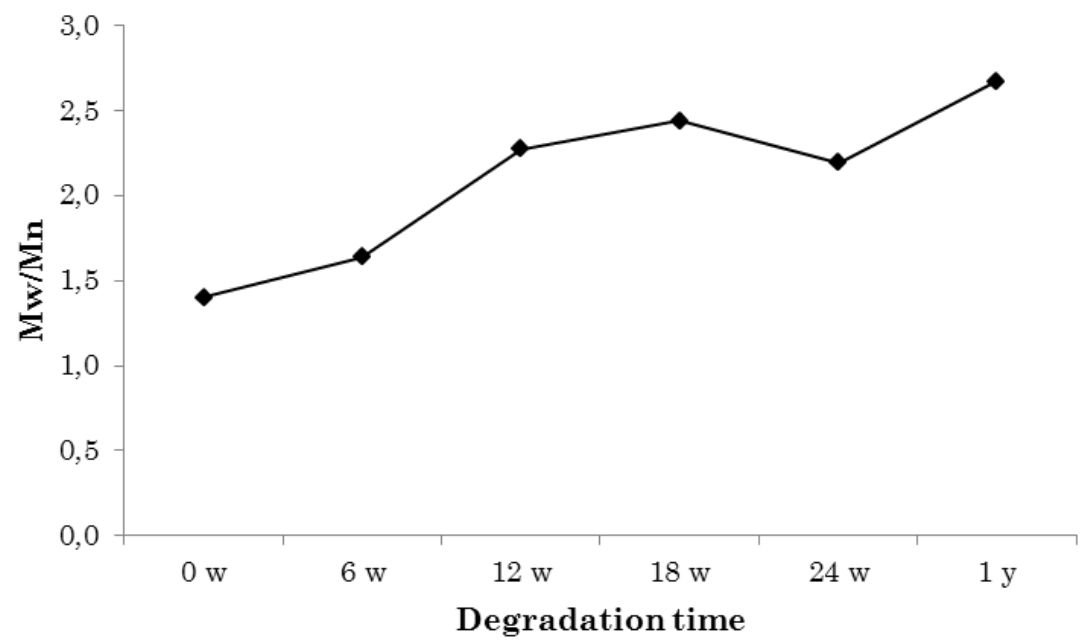

Figure 3-26: Evolution of the polydispersity index (Mw/Mn) as a function of degradation time of PLLA-1:1-15\%-MED scaffolds after $0,6,12,18,24$ weeks and 1 year of static degradation. 


\subsubsection{Thermogravimetric analysis}

The PLLA scaffolds were thermally degraded in order to evaluate the effect of the static degradation in PBS on the thermal stability of the polymer chains. The TGA curves represented in Figure 3-27 represent the residual mass of the scaffolds when they are thermally degraded. The first derivative of mass as a function of the temperature (dw/dT) curves is shown in Figure 3-28. They allow us determining that decomposition of PLLA takes place in mainly only one step; the temperature of the maximum of this step shifts to lower temperatures as the degradation time increases. Persenaire et al. (173) describes the pyrolysis reaction of the ester bond as the first step of thermal degradation of PCL during which volatile components are formed as $\mathrm{H}_{2} \mathrm{O}, \mathrm{CO}_{2}$ and 5-hexenoic acid. A second thermal degradation step is also described as the formation of cyclic monomer as a result of an unzipping depolymerisation process occurring caused by the presence of hydroxyl group (173). In the case of PLLA pyrolysis of the ester bond produces shorter chains that immediately are degraded producing a rapid mass loss traduced in only one peak on the derivative curve. The maximum of this peak for the non-degraded scaffolds takes place at $360^{\circ} \mathrm{C}$ and for the scaffolds one year degraded at $315^{\circ} \mathrm{C}$. This shift could be a consequence of two phenomena: on one hand the presence of a higher proportion of carboxylic and hydroxilic groups in the degraded samples may act as catalyzers of the scission of the ester bonds. On the other hand, as the degraded samples have shorter chains the diffusion of volatile species should be easier and the onset of mass loss is produced at lower temperatures. Indeed, molecular weight distribution curves Figure 3-24 show that as degradation time increases the number of high molecular weigh chains decreases (demonstrated by the lowering of the intensity of the peak) giving place to lower molecular weight 
chains (as shows the broadening to low molecular weight of the distribution curve). The non-hydrolytically degraded scaffolds therefore start to thermally degrade around $300^{\circ} \mathrm{C}$, and those hydrolytically degraded during 1 year start to thermally degrade around $210^{\circ} \mathrm{C}$ (Figure $\left.3-27\right)$.

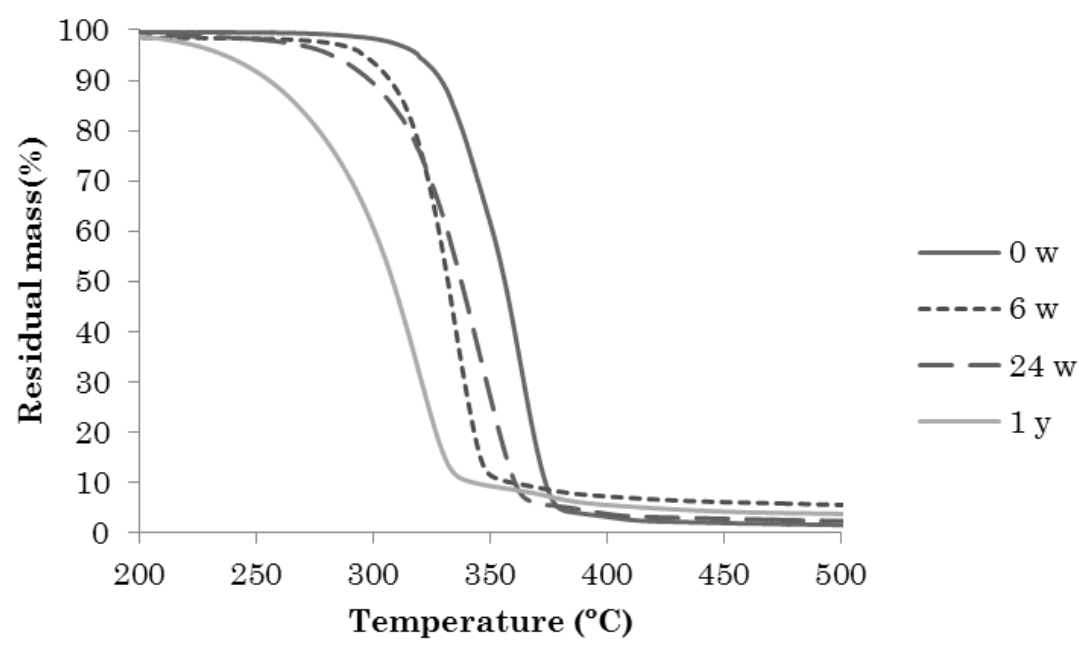

Figure 3-27: Residual mass as a function of temperature of PLLA-1:1-15\%-MED scaffolds after 0, 6, 24 weeks and 1 year of static degradation. 


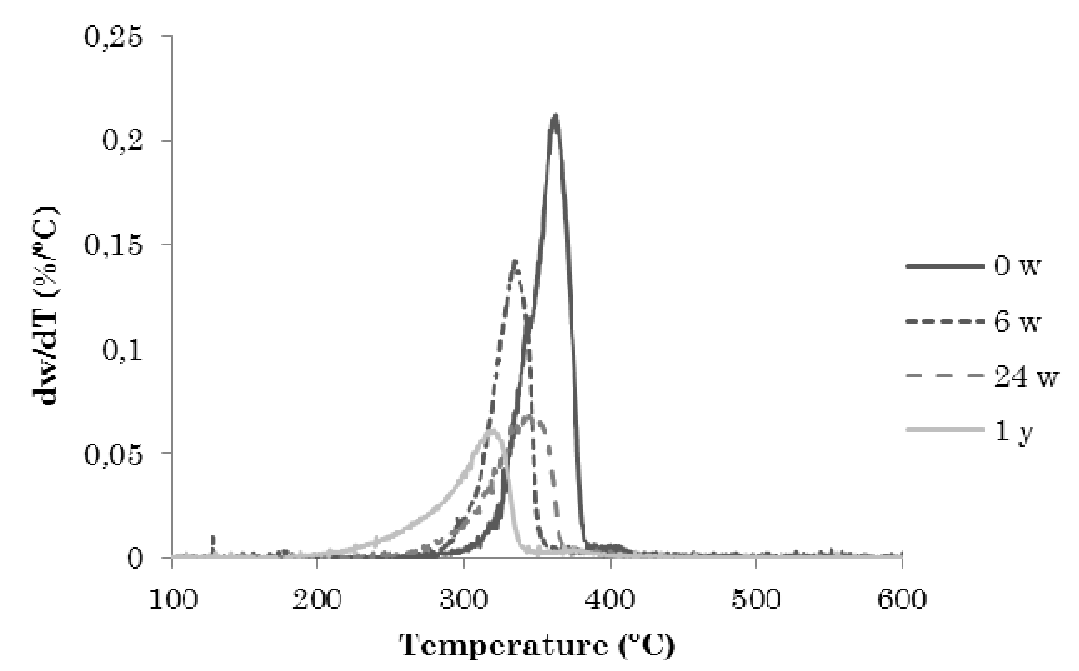

Figure 3-28: First derivative curves of residual mass with temperature (dw/dT) as a function of the temperature of PLLA-1:1-15\%-MED scaffolds after 0, 6, 24 weeks and 1 year of static degradation.

\subsubsection{Fatigue essay}

\subsubsection{Morphology}

The SEM micrographs, Figure 3-29, show the external surface and the cross section of the scaffolds after several weeks of degradation. The deformation of the macroporous structure is most evident in the solicitation direction on the cross-section micrographs. The trabeculae are more and more collapsed as they are degraded. After 6 weeks of dynamic degradation the scaffolds present a compact structure. 


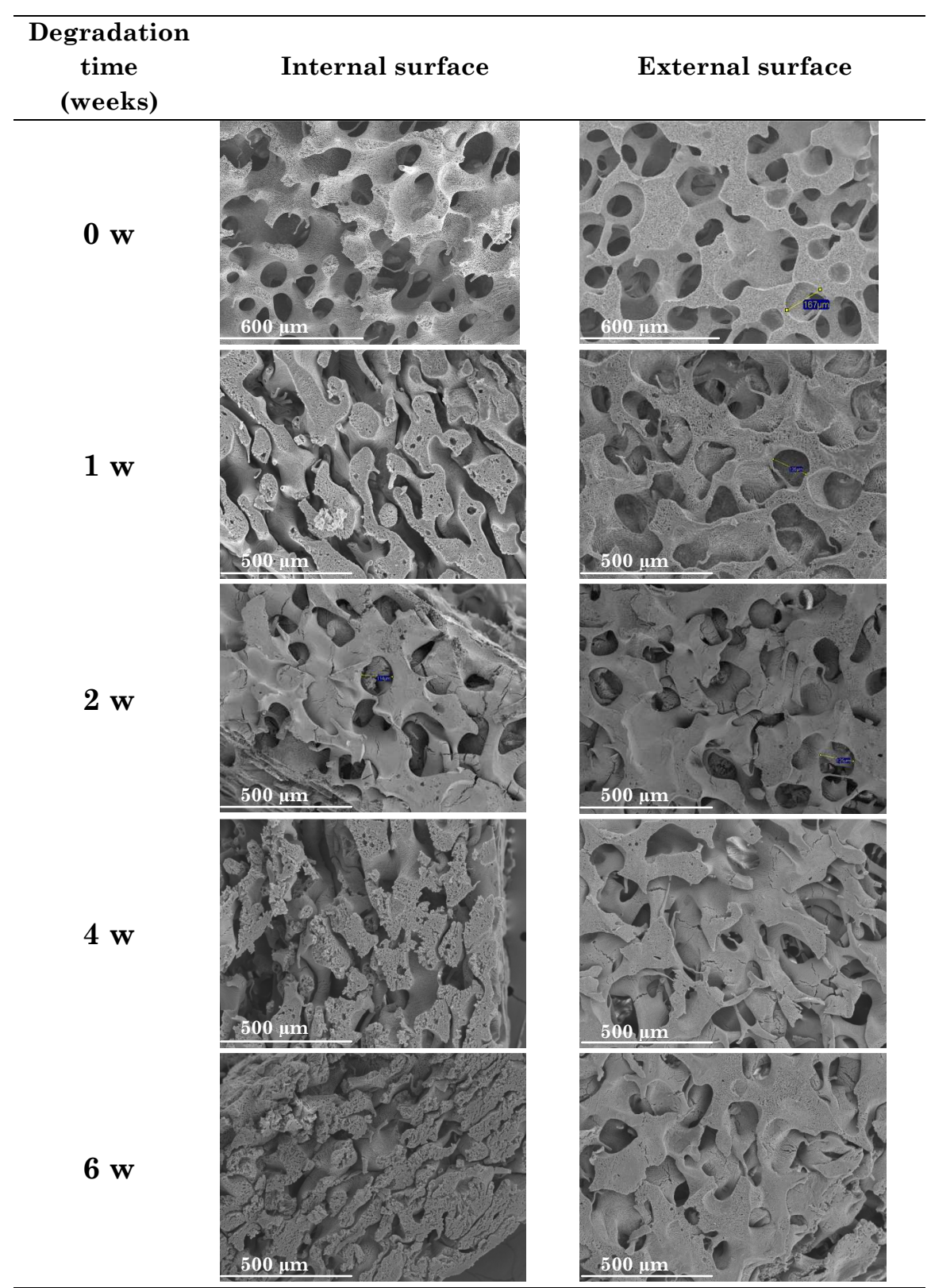

Figure 3-29: SEM micrograph of the PLLA-1:1-15\%-MED scaffolds external and internal surface after $0,1,2,4$ and 6 weeks of dynamic degradation. 


\subsubsection{Mass evolution}

The evolution of the mass of the samples as a function of the degradation time was determined following equation 7, p. 86 . Figure 3-30 shows the mass residue of the different scaffolds after various times of degradation. The mass residue decreases with degradation time only after 2 weeks of degradation. Due to the fact that the observed gain in mass after 1,4 and 6 weeks of degradation could be due to the precipitation of some salts on the surface of the materials we performed an elemental analysis of the samples after the degradation tests.

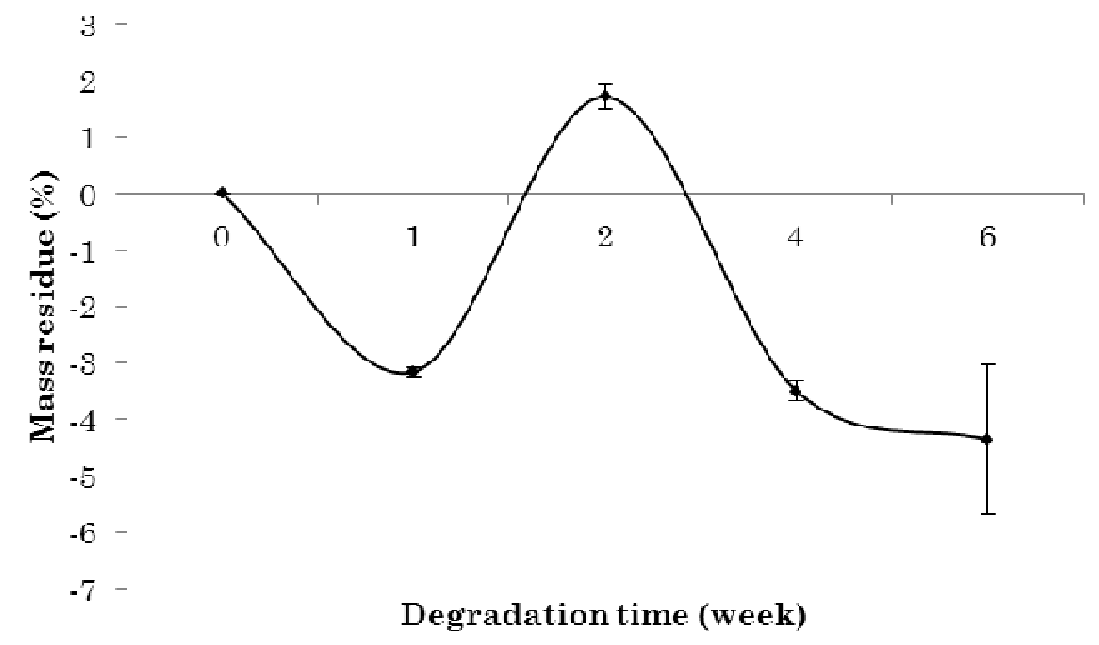

Figure 3-30: Mass residue of the PLLA-1:1-15\%-MED scaffolds after 1, 2, 4 and 6 weeks of fatigue essay. 


\subsubsection{Elemental analysis}

In order to explain why the mass of the sample increased with the degradation process we analysed the surface of the material to detect the eventual nucleation of salts from the PBS. Figure 3-31 presents the results of the elemental analysis carried out on the surface of the scaffolds after 6 weeks of dynamic degradation, where the atomic percentages of the salts detected are represented. The samples were properly rinsed with distilled water before analysis; the same procedure was practiced before weighing them for the determination of the mass after each degradation time. A large amount of $\mathrm{NaCl}$ was found on the surface of the scaffold after fatigue essay. 

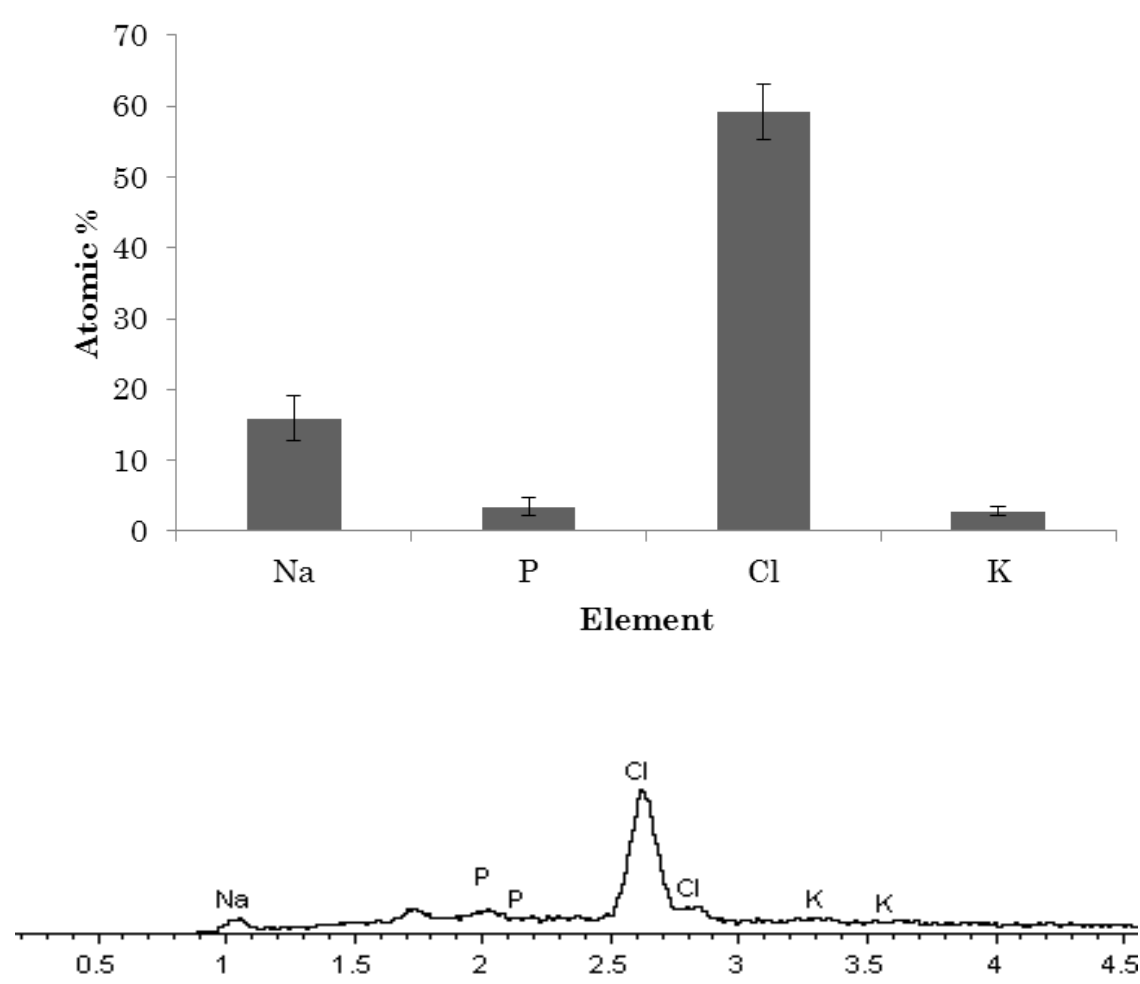

Figure 3-31: (up) Atomic percentage of the element detected on the surface of the samples after 6 weeks of dynamic degradation of the PLLA-1:1-15\%-MED scaffolds and (down) signal obtained during the corresponding elemental analysis.

\subsubsection{Mechanical properties}

The evaluation of the dynamic degradation on the mechanical properties of the scaffolds was done by compression test. In Figure 3-32 and Figure 3-33 it can be observed that there is no elastic zone as the curves start directly in the plateau zone, with a short pore collapse zone. The precision of the equipment used to apply the deformation during the degradation could not avoid the partial pore collapse. 
Consequently, no values of the elastic modulus were obtained. Nevertheless, the effect of degradation on the scaffolds' mechanical properties can be observed. In Figure 3-33 following the horizontal line at a stress of $0.04 \mathrm{MPa}$ it can be determined the corresponding strain as a function of the degradation time. The strain increases from 0.04 for the scaffolds no degraded to 0.13 after 1 week of degradation to a maximum of 0.15 after 2,4 and 6 weeks of degradation. The pore collapse as the scaffolds degraded. After 1 week this zone is shorter and the densification zone is reached at smaller strain (Figure 3-32 and Figure 3-33).

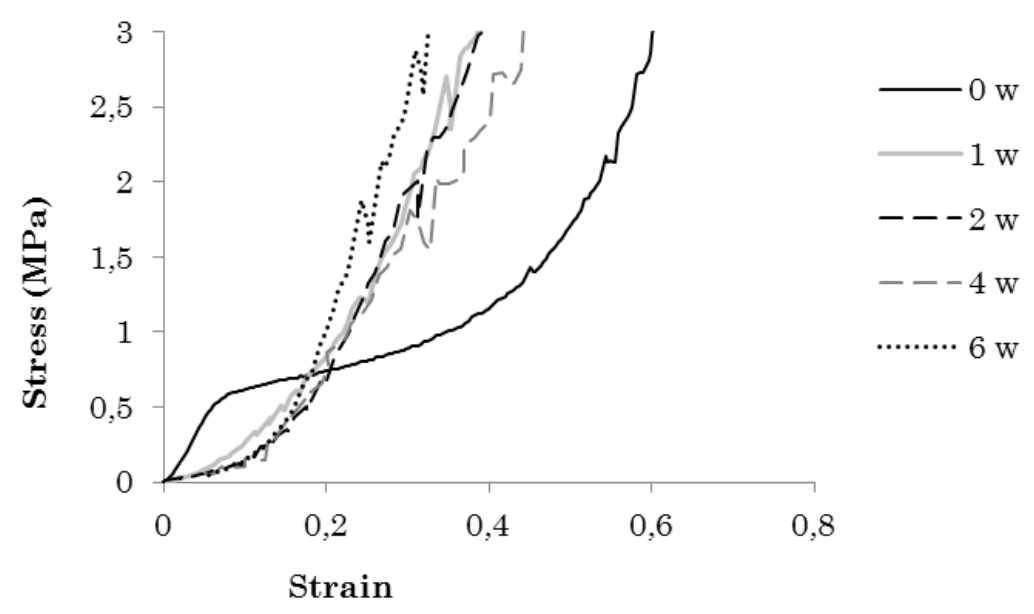

Figure 3-32: Stress - Strain curves of the compression test after $0,1,2,4$ and 6 weeks of dynamic degradation of the PLLA-1:1-15\%-MED scaffolds. 


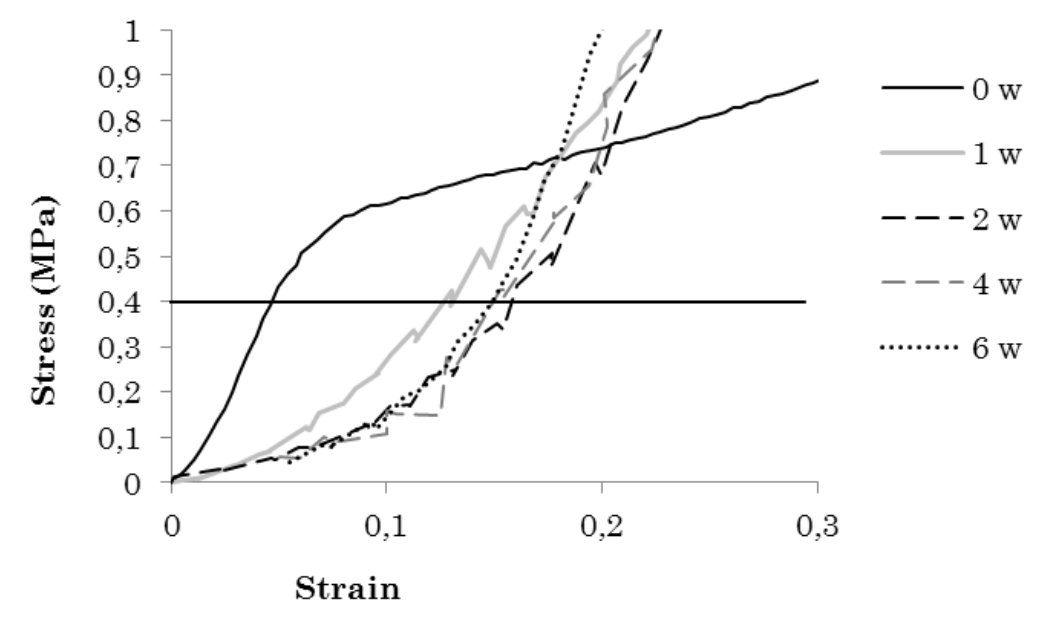

Figure 3-33: Stress - Strain curves of the compression test after $0,1,2,4$ and 6 weeks of dynamic degradation of the PLLA-1:1-15\%-MED scaffolds. The vertical line limiting the elastic zone of the curves of the samples no degraded.

\subsubsection{Calorimetric properties}

Representative curves of the calorimetric properties are represented in Figure 3-34 and compiled in Table 3-8. In these DCS spectra first (1) and second scans (2) can be observed. The curves representative of the first scan shown only glass transition temperature and melting. The second scan shows, in addition to $\mathrm{Tg}$ and $\mathrm{Tm}$, crystallization process around $110^{\circ} \mathrm{C}$ a recrystallization at higher temperature around $160^{\circ} \mathrm{C}$. This phenomenon was also observed in the static degradation study. It also can be observed that the crystallization peak at $110^{\circ} \mathrm{C}$ increases with degradation time. From these curves it was possible to evaluate the evolution of the glass transition temperature (Figure 3-35), the melting temperature maximum (Figure 3-36) and the crystallinity (Figure 3-37). It can be noticed that the glass transition temperature, melting 
temperature and the crystallinity of the second heating is smaller than the first one. The glass transition temperature for both scans remains constant when the scaffolds get more and more degraded. The decrease of the melting temperature when the time of degradation increases is more pronounced during the second heating than the first one, in particular after the first week of degradation; falling from 183 to $179^{\circ} \mathrm{C}$ after the first scan, between 0 and 6 weeks of degradation.

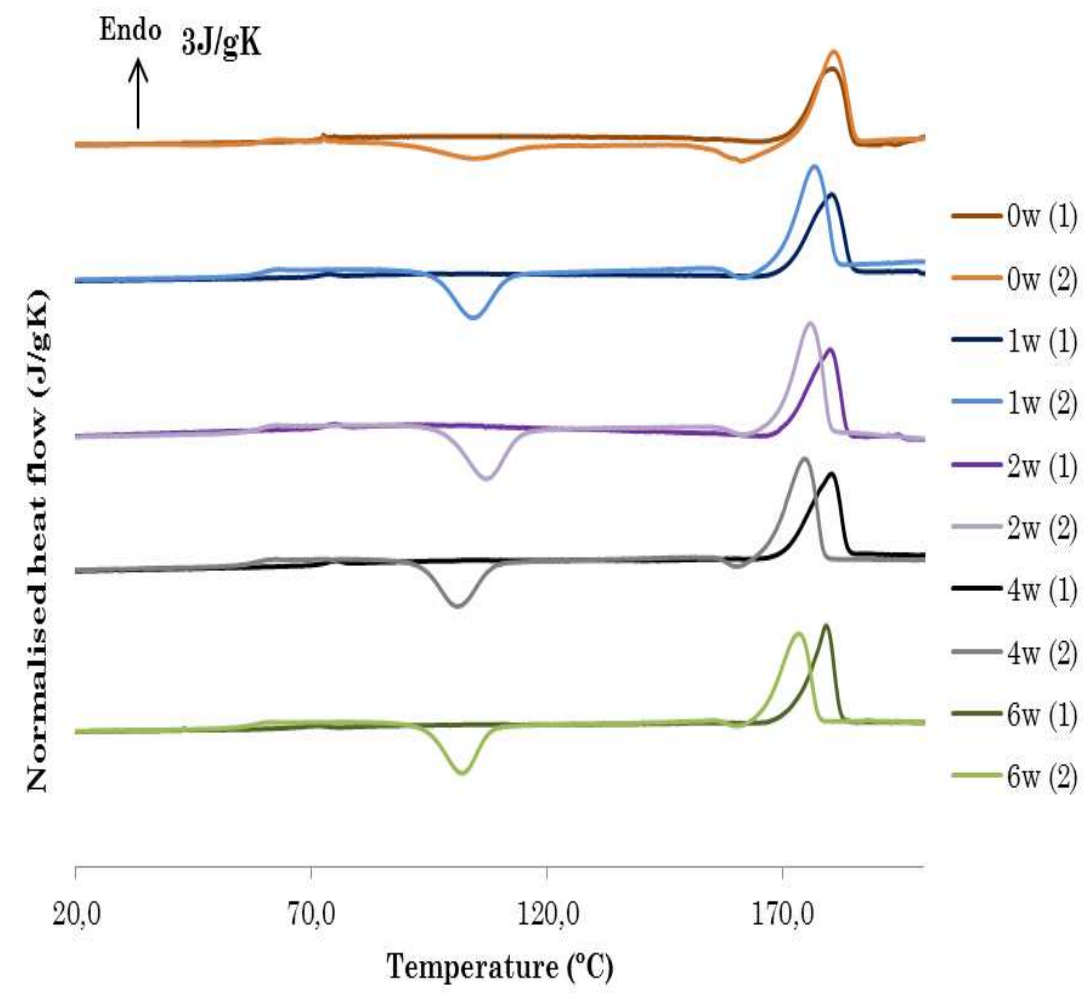

Figure 3-34: Comparison of the DSC first (1) and second (2) heat scans after $0,1,2,4$ and 6 weeks of dynamic degradation. of the PLLA-1:1-15\%-MED scaffolds The heat flow was normalised by the mass of the samples and the heating rate at $10^{\circ} \mathrm{C} / \mathrm{min}$. The arrow mark represents 3 units of normalized heat flow in $\mathrm{J} / \mathrm{gK}$. 


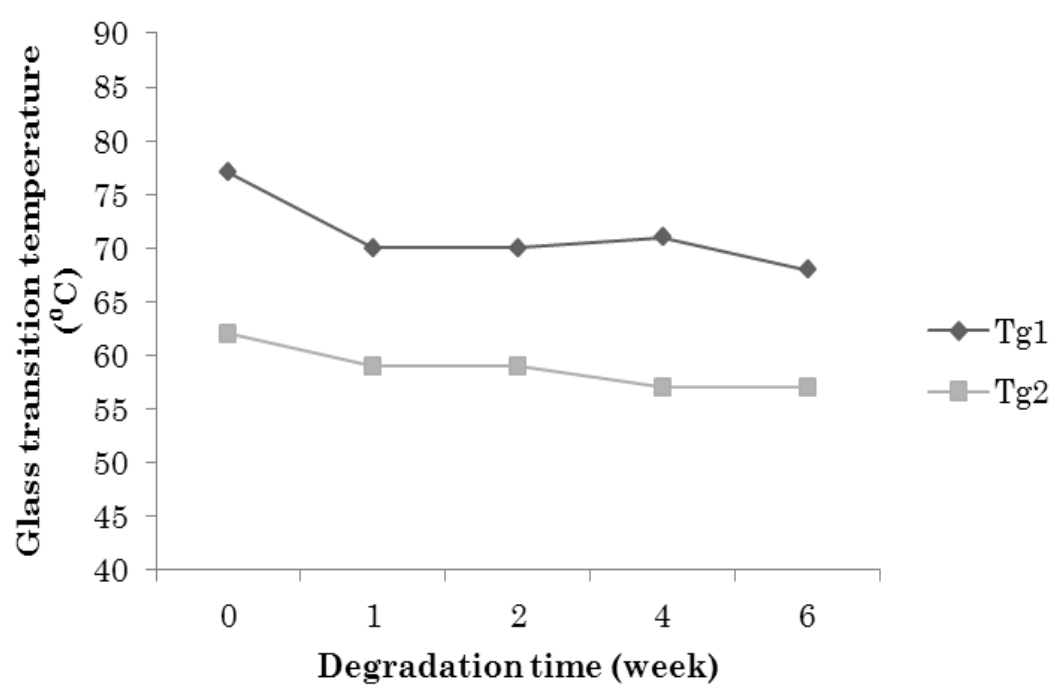

Figure 3-35: Glass transition temperature after the first scan $\left(\mathrm{Tg}_{1}\right)$ and the second scan $\left(\mathrm{Tg}_{2}\right)$ of the PLLA-1:1-15\%-MED scaffolds after $0,1,2,4,6$ weeks of dynamic degradation.

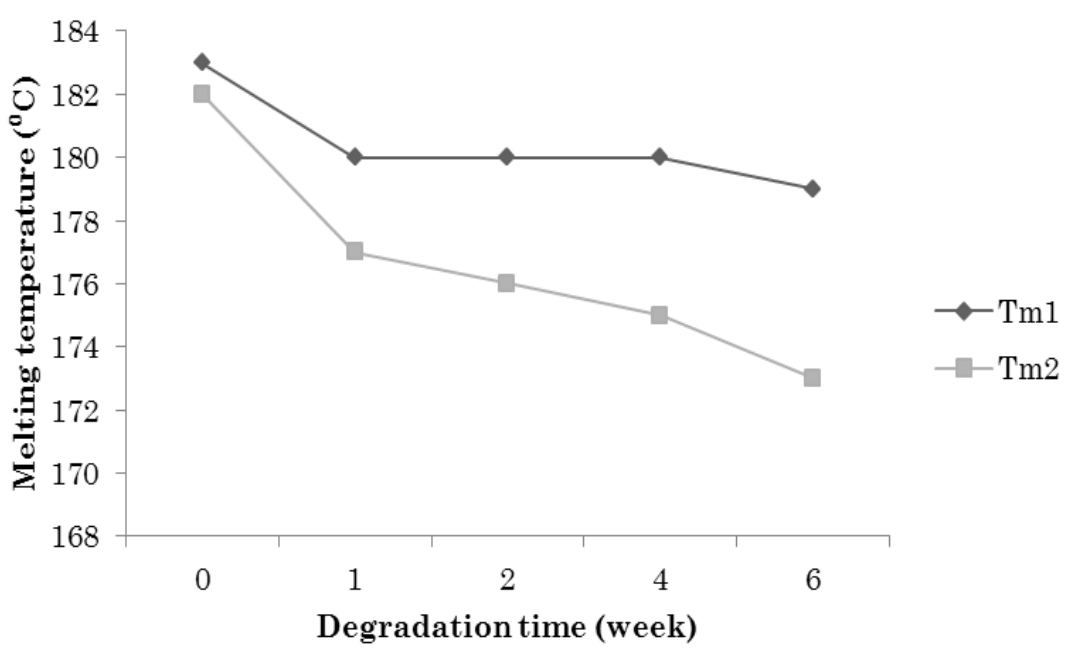

Figure 3-36: Maximum of melt temperature after the first scan $\left(\operatorname{Tm}_{1}\right)$ and the second scan $\left(\mathrm{Tm}_{2}\right)$ of the PLLA-1:1-15\%MED scaffolds scaffolds after $0,1,2,4,6$ weeks of dynamic degradation. 


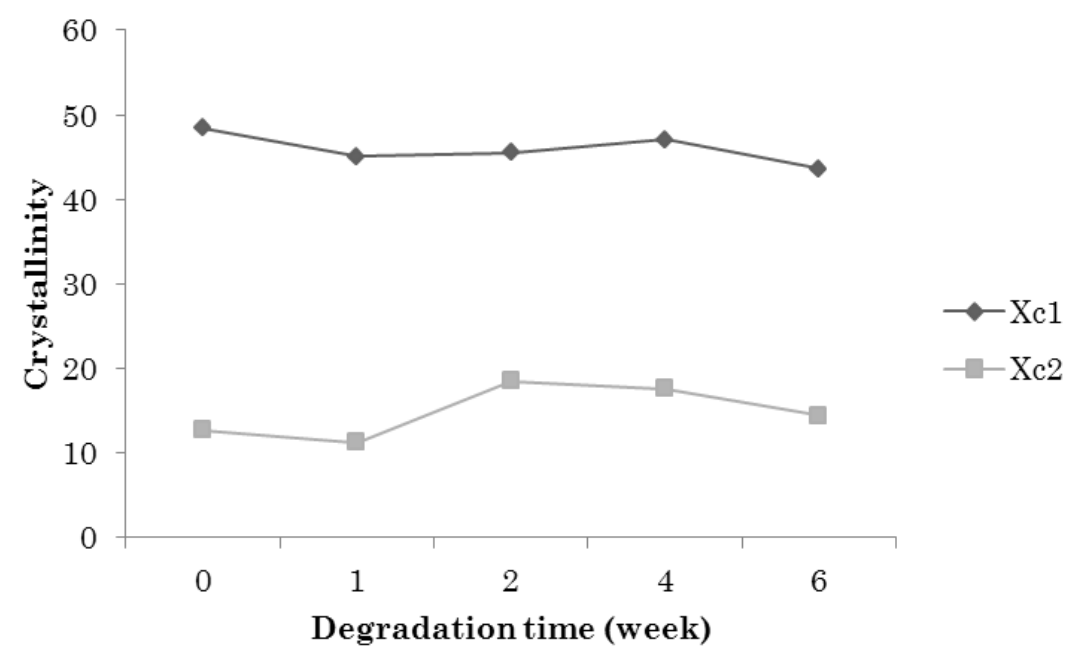

Figure 3-37: Crystallinity after the first scan $\left(\chi c{ }_{1}\right)$ and the second scan $\left(\chi c_{2}\right)$ of the PLLA-1:1-15\%-MED scaffolds after 0 , $1,2,4$ and 6 weeks of dynamic degradation.

Table 3-8: Crystallinity and glass transition temperature after the first (1) and second (2) DSC scan of the PLLA-1:115\%-MED scaffolds after different time of dynamic degradation in PBS at $37^{\circ} \mathrm{C}$.

\begin{tabular}{|c|c|c|c|c|}
\hline \multirow[t]{2}{*}{$\begin{array}{c}\text { Time of } \\
\text { Degradation }\end{array}$} & \multicolumn{2}{|c|}{$\chi_{\mathrm{c}}(\%)$} & \multicolumn{2}{|c|}{$\begin{array}{c}\text { Glass transition } \\
\text { Temperature }\left({ }^{\circ} \mathrm{C}\right)\end{array}$} \\
\hline & $\chi_{\mathrm{c} 1}$ & $\chi_{\mathrm{c} 2}$ & $\mathrm{Tg}_{1}$ & $\mathrm{Tg}_{2}$ \\
\hline $0 \mathrm{w}$ & 48 & 13 & 77 & 62 \\
\hline $1 w$ & 45 & 11 & 70 & 59 \\
\hline $2 w$ & 45 & 19 & 70 & 59 \\
\hline $4 \mathrm{w}$ & 47 & 18 & 71 & 57 \\
\hline $6 \mathrm{w}$ & 44 & 14 & 68 & 57 \\
\hline
\end{tabular}




\subsubsection{Gel permeation chromatography}

A small fraction of the polymer chains are affected by the dynamic degradation as the molecular weight decreased slightly after one week and then remains constant (Figure 3-39) and the polydispersity index increased (Figure 3-40). This phenomenon was also observed in Figure 3-38 as the principal peak was reduced between week 0 and 1 week. The small decrease in molecular weight was similar to the one observed for the static degradation at 6 weeks.

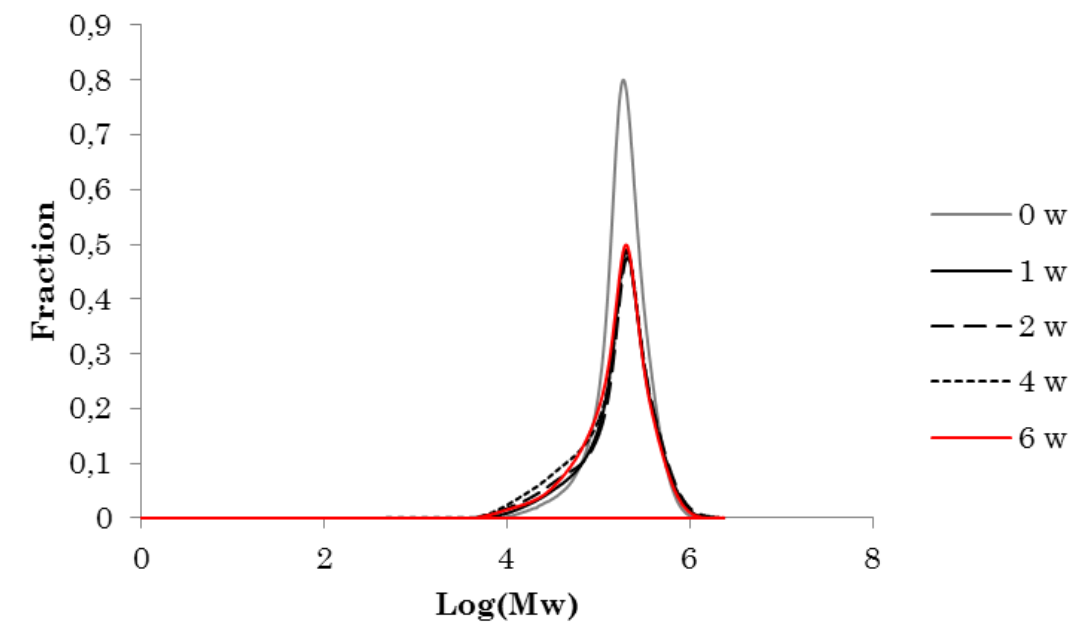

Figure 3-38: Gel Permeation Chromatography curves representing the fraction of polymer chain as a function of $\log (\mathrm{Mw})$ after $0,1,2,4$ and 6 weeks of dynamic degradation. 


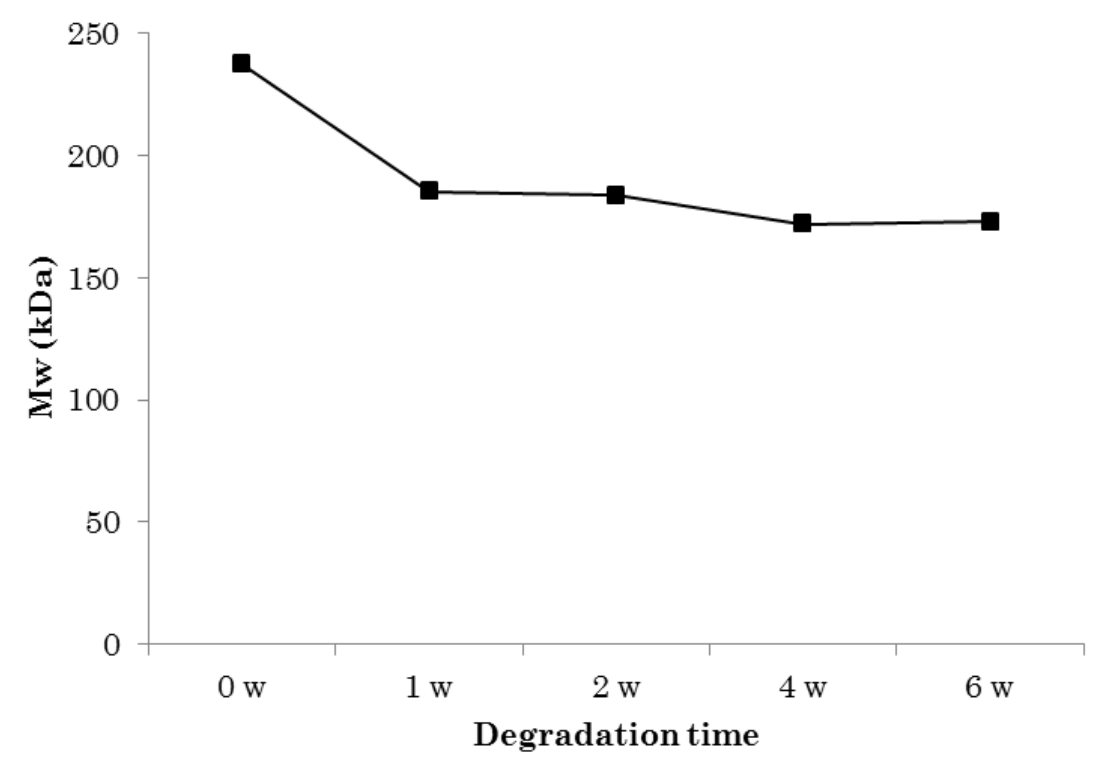

Figure 3-39: Average molecular weight of the PLLA-1:1-15\%MED scaffolds after $0,1,2,4$ and 6 weeks of dynamic degradation.

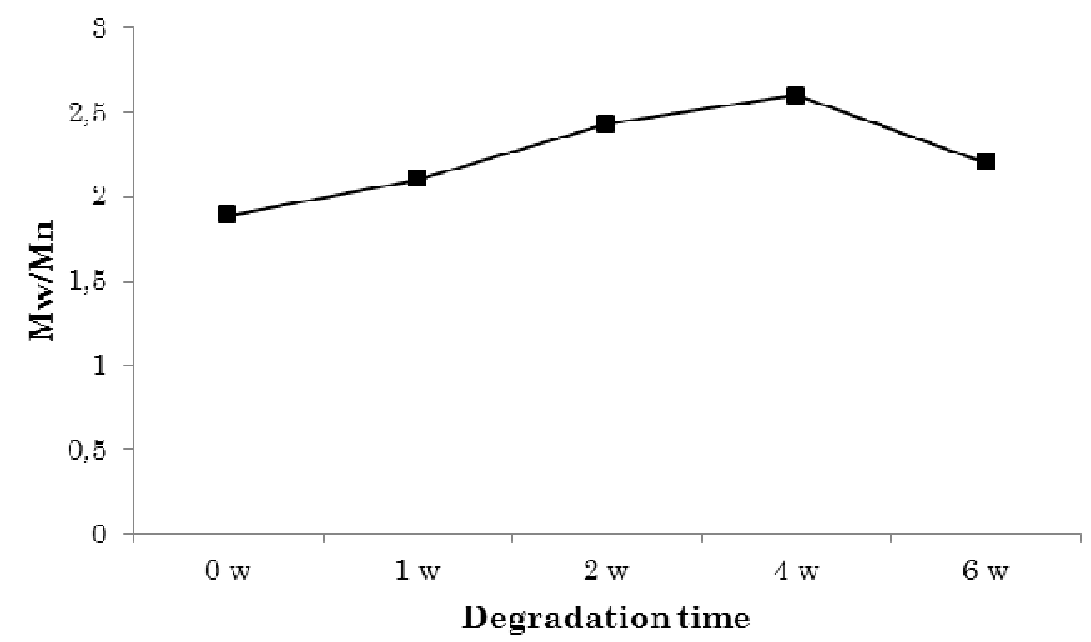

Figure 3-40: (Mw/Mn) of the PLLA-1:1-15\%-MED scaffolds after $0,1,2,4$ and 6 weeks of dynamic degradation. 


\subsubsection{Thermogravimetric analysis}

As noticed in the static degradation study, thermal degradation was performed in order to determine the effect of the fatigue essay on the thermal resistance of the polymer chains. The results of this test are shown in Figure 3-41 and Figure 3-42. The derivative mass loss peak lowers its intensity and shifts from $360^{\circ} \mathrm{C}$ to $315^{\circ} \mathrm{C}$ after 1 week of dynamic degradation (Figure 3-42), a phenomena that already occurred on the static degradation experiments. Furthermore the thermal degradation stability is decreased as the degradation curves onsets (Figure 3-41) lower from $320^{\circ} \mathrm{C}$ to $240^{\circ} \mathrm{C}$ after 6 weeks of degradation. This indicates the formation of smaller polymer fragments when scaffolds degraded in PBS.

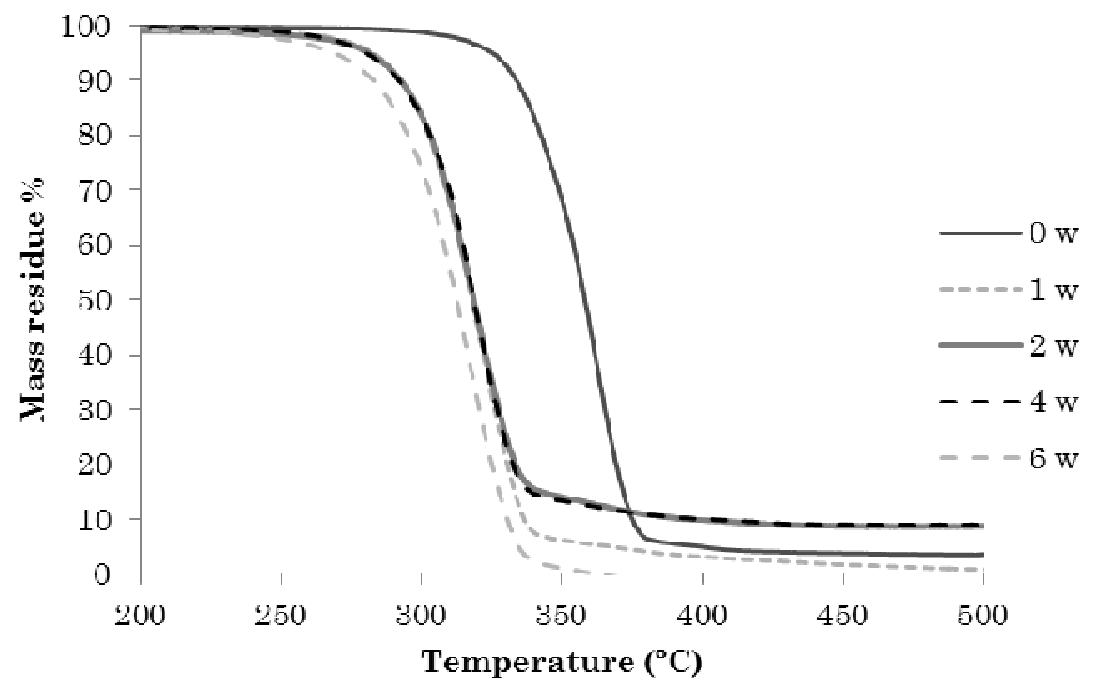

Figure 3-41: Mass residue percentage as a function of the temperature of the PLLA-1:1-15\%-MED scaffolds after 0, 1, 2, 4 and 6 weeks of dynamic degradation. 


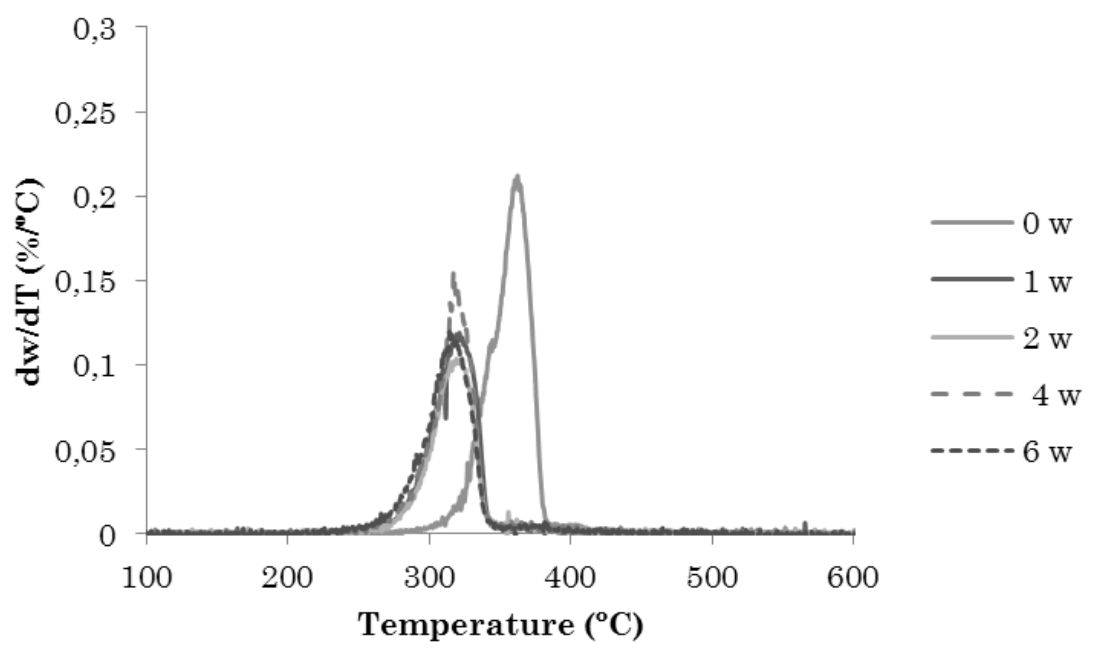

Figure 3-42: Evolution if the first derivative of weight as a function of the temperature curves of the PLLA-1:1-15\%-MED scaffolds after $0,1,2,4$ and 6 weeks of dynamic degradation. 



\subsection{REINFORCEMENT AND BIOACTIVITY OF THE MATERIAL}

The polymeric scaffolds selected on section 4.1.2, p.176, which will be the basis for the design of the construct for osteochondral scaffolds, are much more compliant than bone. This constraint is not only due to the polymeric character of PLLA but also to its high porosity. The aim of this chapter is to look for the best strategy to reinforce the scaffolds and at the same time to confer an adequate bioactivity to the bony part of the construct. The aim is to try to have a part of the construct with mechanical properties more similar to subchondral bone, with a good anchorage to the host tissue due to its high bioactivity. The strategy we followed was to add bioactive nanoparticles to the organic PLLA matrix. First, we prepared $2 \mathrm{D}$ composite membranes as an easy way to test the viability of our approach, and then $3 \mathrm{D}$ scaffolds were produced in order to approximate working conditions. The same brands of PLLA as in section 3.1, p.63, were used: industrial PLLA and the medical PLLA. The influence of the inherent properties and the concentration of the nanoparticles used as filler on the hybrid structure were studied. The fabrication protocol was optimized for our specific objectives. Two kinds of fillers have been incorporated: commercial hydroxyapatite (HAp: $\left.\mathrm{Ca}_{10}\left(\mathrm{PO}_{4}\right)_{6}(\mathrm{OH})_{2}\right)$ nanoparticles and laboratory produced nanoporous silica $\left(\mathrm{SiO}_{2}\right)$ particles. HAp is naturally present in bone tissue and its bioactive properties have been widely tested. More recently the use of bioactive silicates has been proposed as an alternative to apatites due to the interesting mechanical properties obtained with them $(152,153)$. The hybrids were thoroughly characterized with emphasis on the reinforcement and bioactive potential of these inorganic fillers. Membranes from a solution of PLLA in dioxane with a $15 \%$ wt. of PLLA-MED and different quantities of HAp 
nanoparticles $(0,5,10$ and $15 \%$ wt. referred to the mass of PLLA) were fabricated. Then a bioactivity test was performed by soaking them in a simulated body fluid solution (SBF) for 0 , 7, 14 and 21 days. The effect of a surface pre-treatment with oxygen plasma on the bioactivity of pure PLLA and composites was also studied.

Then this study was extrapolated to scaffolds. Scaffolds in which nanoparticles of HAp and silicate were incorporated to the polymeric matrix to enhance their bioactivity were fabricated. The bioactivity test used in the case of the scaffolds is the same as the one used for the membranes. In addition the effect of a new type of filler is evaluated on the bioactivity of the samples. Sonication is the method used to incorporate the filler to the matrix. In this way the effect on the interaction between the filler and the polymeric matrix on the mechanical properties can be studied.

\subsubsection{D hybrid structure}

\subsubsection{Morphology}

Figure 3-43 and Figure 3-44 present the surface of the membranes observed by scanning micrograph. Figure 3-43 shows the membranes filled with 0, 5, 10 and $15 \%$ wt. in HAp nanoparticles with and without plasma exposure. Figure 3-44 shows the surface of the membrane filled with $15 \%$ of HAp particles at two different magnifications. Figure 3-45 shows the surface of the membranes with and without exposure to plasma, as the pictures were done at high magnification it is possible to evaluate the effect of the plasma treatment on the surface of the membranes.

The membranes were fabricated using freeze-extraction process to generate the microporosity. No porogen was added as the plan was to build a $2 \mathrm{D}$ structure. The nanoparticles of filler were added to the solvent before dissolving the polymer in dioxane. The morphology observed in Figure 3-43 is similar 
to that observed on the pore walls of the scaffolds prepared in section 3.1, p.63, by freeze-extraction and particle leaching. The micropores are homogenously dispersed on the surface of the membranes. To confirm repeatability of the technique, 3 different membranes were fabricated and observed by SEM. All membranes exhibited the same morphology. The pores are produced by the phase separation when both dioxane and PLLA crystallise from the solution. 


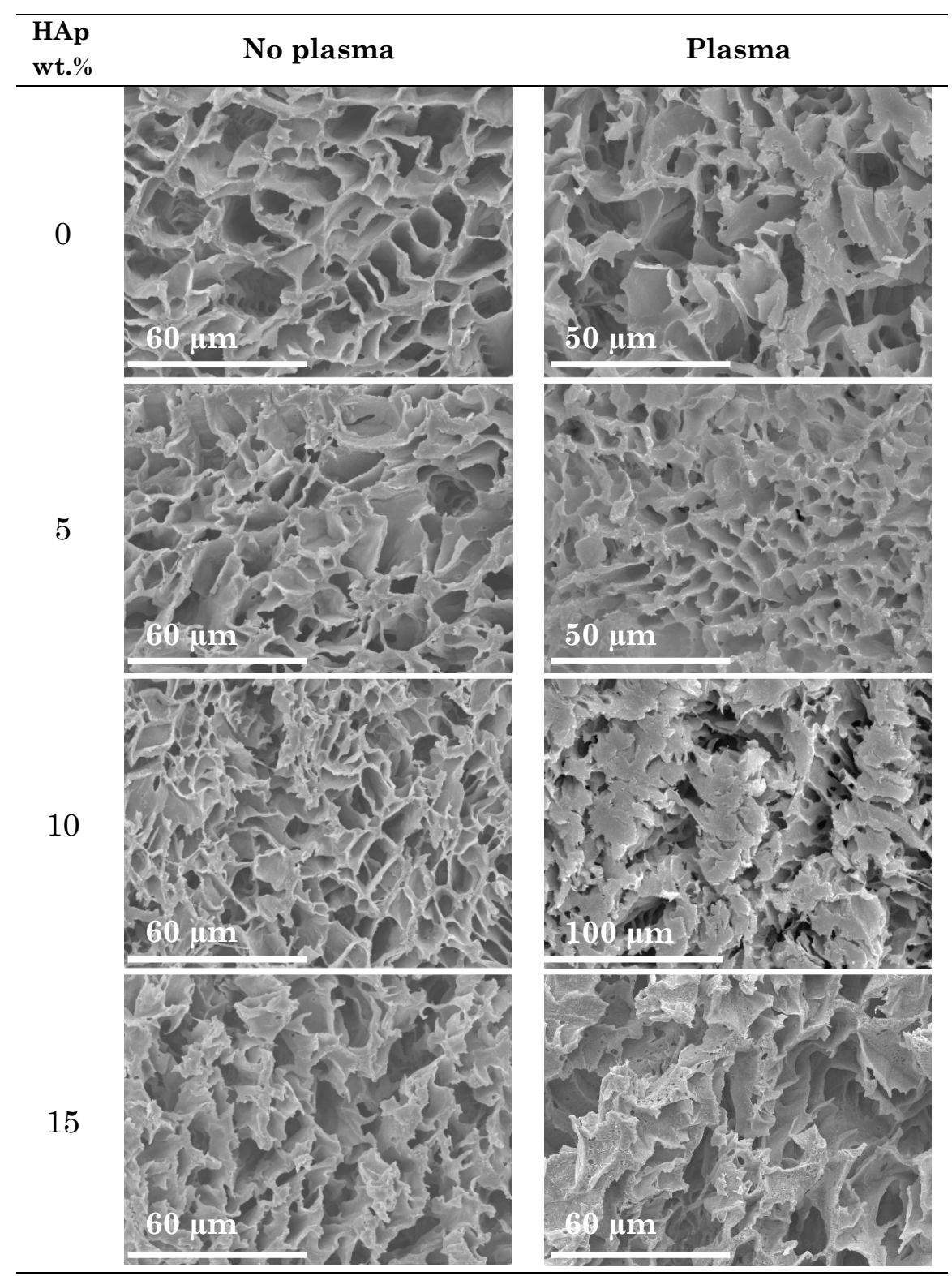

Figure 3-43: Scanning electron microphotographs of the surface of the PLLA membrane prepared with a PLLA/dioxane solution with a $15 \%$ wt. of polymer by freeze extraction and filled with $0,5,10$ and 15 wt. $\%$ of nanoparticles of hydroxyapatite after plasma treatment (right) and without (left). 
The micropore dimension was measured for the membranes exposed to plasma $12.3 \pm 4.6 \mu \mathrm{m}$ and $12.9 \pm 4.1 \mu \mathrm{m}$ for those that were not. They present a pore dimension greater than the one obtained for the scaffolds but of the same order of magnitude. The dimensions of the pores are not influenced by the content in filler. The structure of the microporosity as shown in Figure 3-43 does not present any significant changes when the filler content increases. The pictures present some differences but due to the way the membranes were cut. It was not possible to cut the membranes through the same angle.

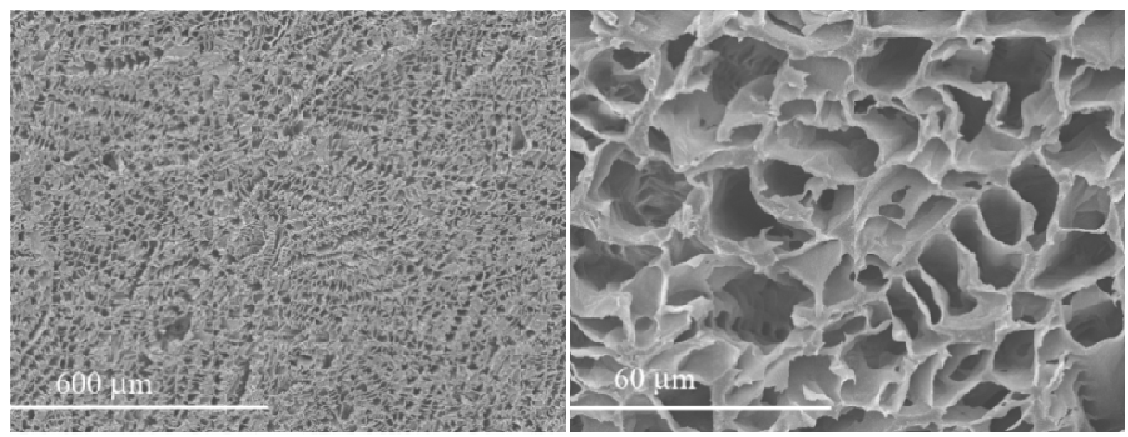

Figure 3-44: Scanning electron microphotographs of the surface of the PLLA membrane prepared with a PLLA/dioxane solution of with a $15 \%$ wt. of polymer by freeze extraction without plasma: (left) low magnification micrograph and (right) high magnification micrograph.

The morphology of the membrane seems to not be affected by plasma treatment. After oxygen plasma treatment, as shown in Figure 3-45 some kind of abrasion defects can be seen on the surface of the membranes, which does not appear in the membranes without plasma treatment. Surface abrasion was already found in plasma treated PLLA by $\mathrm{Qu}$ et al. (120) and in our study this abrasion allows HAp particles to appear at the pores walls. HAp nanoparticles cannot be clearly seen by SEM, especially in the untreated membranes, probably because a layer of PLLA covers them. However, EDS analysis 
was able to show $\mathrm{Ca}$ and $\mathrm{P}$ peaks, characteristic of the HAp components, as shown by the insets in Figure 3-45. The EDS analysis was repeated three times in different zones for three different samples, and all the signals present the same behaviour, showing that particle dispersion is homogenous.

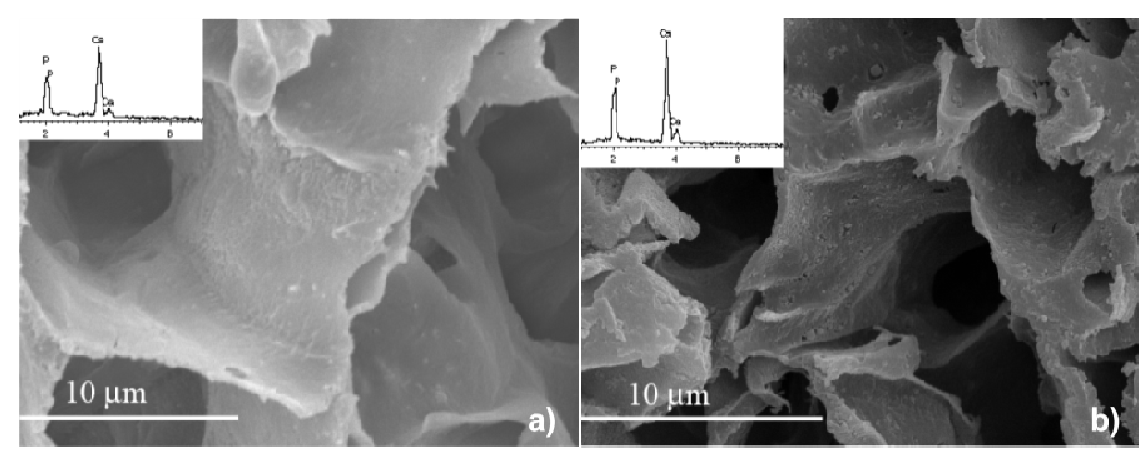

Figure 3-45: Scanning electron micrographs and Energy dispersive spectroscopy analysis (as inset in the micrographs) of PLLA/HAp nano-composites with $10 \% \mathrm{wt}$. of HAp: (a) without plasma treatment, (b) after plasma treatment.

\subsubsection{Nanoparticles content evaluation}

In order to evaluate the effective content of nanoparticles incorporated in the membranes, 3 membranes of each composition were calcinated up to $1000^{\circ} \mathrm{C}$. In this way, the mass of the polymer was eliminated from the composite membrane, allowing the residual mass to be used to evaluate the real content of nanoparticles in the membranes. In Table 3-9 results of the content of measured nanoparticles are compiled and compared to the nominal ones. The calcination experiments showed that the amount of HAp incorporated into the membranes is similar to but lower than the amount of HAp dispersed in the PLLA solution. The more HAp was used to fill the membranes the less is really incorpoarated. The 
membranes that are filled with $5 \%$ wt. of HAp are really filled with $4.45 \pm 0.10 \%$ wt., and those supposed to be filled with 15 $\%$ wt. are really filled with $11.82 \pm 1.12 \%$ wt. The more HAp that is incorporated to the membranes, the more difficult it is to control the exact content in filler.

Table 3-9: Effective nanoparticles mass content derived from the calcination experiments compared to the nominal ones in the PLLA/HAp membranes.

\begin{tabular}{ccc}
\hline $\begin{array}{l}\text { Nominal } \\
\text { HAp content (\%) }\end{array}$ & $\begin{array}{l}\text { \% of } \\
\text { incorporated }\end{array}$ & $\begin{array}{c}\text { Deviation (\%) } \\
\text { to the mass of } \\
\text { HAp } \\
\text { incorporated }\end{array}$ \\
\hline $\mathbf{0}$ & - & - \\
$\mathbf{5}$ & $4.45 \pm 0.10$ & -10.9 \\
$\mathbf{1 0}$ & $8.85 \pm 0.74$ & -11.5 \\
$\mathbf{1 5}$ & $11.82 \pm 1.12$ & -21.2 \\
\hline
\end{tabular}

\subsubsection{Differential scanning calorimetry}

Figure 3-46 shows the first and second heating of the diferent membranes filled with nanoparticles of HAp, and Table 3-10 compiled the transition temperature obtained from the DSC scans. The first heating scan shows the glass transition with a large overshoot characteristic of the physical ageing (or structural relaxation) process suffered by the polymer during annealing at $40^{\circ} \mathrm{C}$ while drying in vacuum. The cold crystallisation peaks appear after glass transition. These cold crystallisation peaks do not appear in the case of the scaffolds as it was observed in section 3.2.1.5, p.92, one factor that can explain this peak appearance is that the membranes are not dipped in ethanol at $40^{\circ} \mathrm{C}$ as non porogen particle is incorporated in the fabrication process. The ethanol bath at $40^{\circ} \mathrm{C}$ permits crystallization of the polymer. These exothermal peaks appear at lower temperatures as the HAp nanoparticle 
content increases (Figure 3-46 - left). Another crystallisation process appears at higher temperatures that is characteristic of highly nucleated samples that crystallize at very low temperatures (130). Finally the melting process takes place at a temperature independent of sample composition. The second scan is representative of the samples crystallized after melting. Here the endothermal overshoot due to physical ageing disappears since the sample was not annealed below the glass transition (Figure 3-46 - right). The crystallization and melting follow a trend similar to that of the first scan, although the crystallization peaks are clearly shifted towards higher temperatures.

The determination of crystallinity was done by an integration of the heat flow trace with respect to a linear baseline that intersects a point immediately above the glass transition and another one after melting. If the sample was completely amorphous at the beginning of the heating scan the area between the thermogram and the baseline would be zero. The calculus becomes quite uncertain since the temperature interval to which it is extended is very broad and can easily lead to small errors in the determination of the baseline used in the integration (for instance for small curvatures in the baseline of the calorimeter). This difficulty is especially clear in the first scan when the thermogram starts deviating towards the exothermal side immediately above the glass transition (Figure 3-46 - left), which impedes any enthalpy calculation, and so the determination of the crystallinity after the first scan. The melting behaviour does not present any changes between the different samples and both scans; the peaks appear at the same temperature.

The data relative to the glass transition temperature of the first and second scans and the crystallinity calculated after the second scan are compiled in Table 3-10. Other researchers have studied the effect of freeze-extraction on PLLA/ PCL mixture and the crystallinity obtained was $19 \%$ for the 
samples of the same PLLA used in our case (177). In the second scan crystallinity values between 6 and 20\% were found but the HAp content dependence was non-systematic. The onset of the glass transition seems to be independent to the filler content (Figure 3-45). A slight decrease of the enthalpic glass transition temperature with the HAp content is observed.
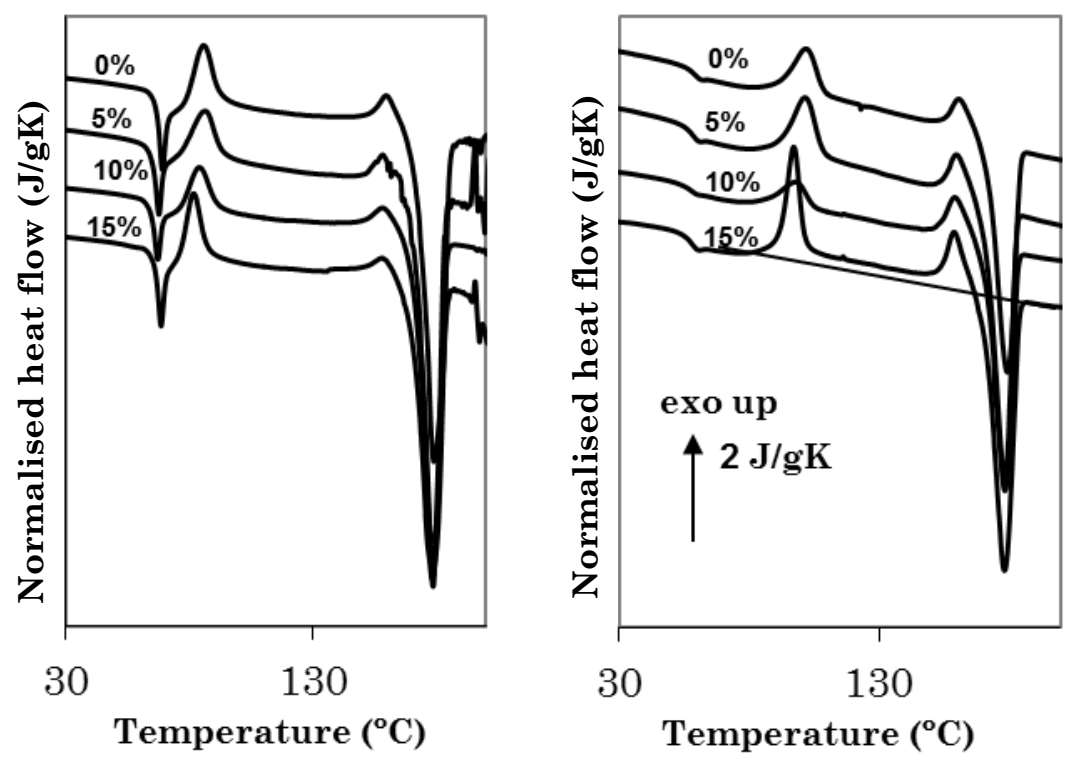

Figure 3-46: First DSC heating scan of PLLA/HAp nanocomposites with different filler content (left), and second DSC heating scan (right), both obtained at a heating rate of $10^{\circ} \mathrm{C} / \mathrm{min}$. 
Table 3-10: (onset) Glass transition temperature and crystallinity of the membranes filled with $0,5,10$ and $15 \%$ wt. of nanoparticles of HAp.

\begin{tabular}{cccc}
\hline \multirow{2}{*}{ HAp content (\%) } & $\begin{array}{c}\text { Tg onset }\left({ }^{\circ} \mathbf{C}\right) \\
\text { first scan }\end{array}$ & $\begin{array}{c}\text { Enthalpic Tg }\left({ }^{\circ} \mathbf{C}\right) \\
\text { second scan }\end{array}$ & 霆 (\%) \\
\hline $\mathbf{0}$ & 51 & 36 & 10.4 \\
$\mathbf{5}$ & 48 & 38 & 6.2 \\
$\mathbf{1 0}$ & 51 & 38 & 19.9 \\
$\mathbf{1 5}$ & 49 & 37 & 5.9 \\
\hline
\end{tabular}

\subsubsection{Dynamic mechanical spectroscopy}

The dynamic mechanical properties at tension mode of the membranes were determined. The samples were first subjected to heating from 0 to $120^{\circ} \mathrm{C}$ in the first measuring scan. The graph on the left DMS curves in Figure 3-47 show the temperature dependence of the storage modulus (E') for the first and second scans of the membrane with a $10 \% \mathrm{wt}$. of HAp and the curves on the right show E' of the second scan for all the composites. The E' curve for the first scan shows a sharp decrease starting close to the calorimetric glass transition. The shape of the curve with a change of slope in the middle of the relaxation indicates that crystallization starts during the scan itself. This makes the values measured at temperatures above Tg quite dependent on thermal history in the scan itself. The modulus in the glass region measured in the first scan increases with the HAp content of the composites (Table 3-11). To compare the behaviour of the different nano-composites with a more reproducible structure, samples were annealed at $120^{\circ} \mathrm{C}$ for one hour to allow crystallization and cooled to $0^{\circ} \mathrm{C}$ to start the second scan. Thus, the second scan corresponds to higher crystallinity samples. The main relaxation in the second scan is shifted to higher temperatures with respect to the first scan. This behaviour is expected due to the increase of glass transition 
temperature of the amorphous phase with the increase of crystal fraction. The value of $\mathrm{E}$ ' in the glassy region increases with respect to the first scan due to the higher fraction of PLLA crystals. The second scan shows that the value of E' increases with the content of filler incorporated to the matrix both at low temperatures when the amorphous phase is in the glassy state and after the main relaxation when it is in the rubber-like state (Table 3-11). Nevertheless, the increment although systematic and significant is not the one expected for a nanocomposite with a stiff nano reinforcing filler. In fact, as shown in Table 3-11, the value of the elastic modulus in the membrane with the highest HAp content does not reach twice that of the PLLA matrix. This fact must be ascribed to a poor interface adhesion between polymer matrix and filler, and results in a limited stress transmission between phases. 

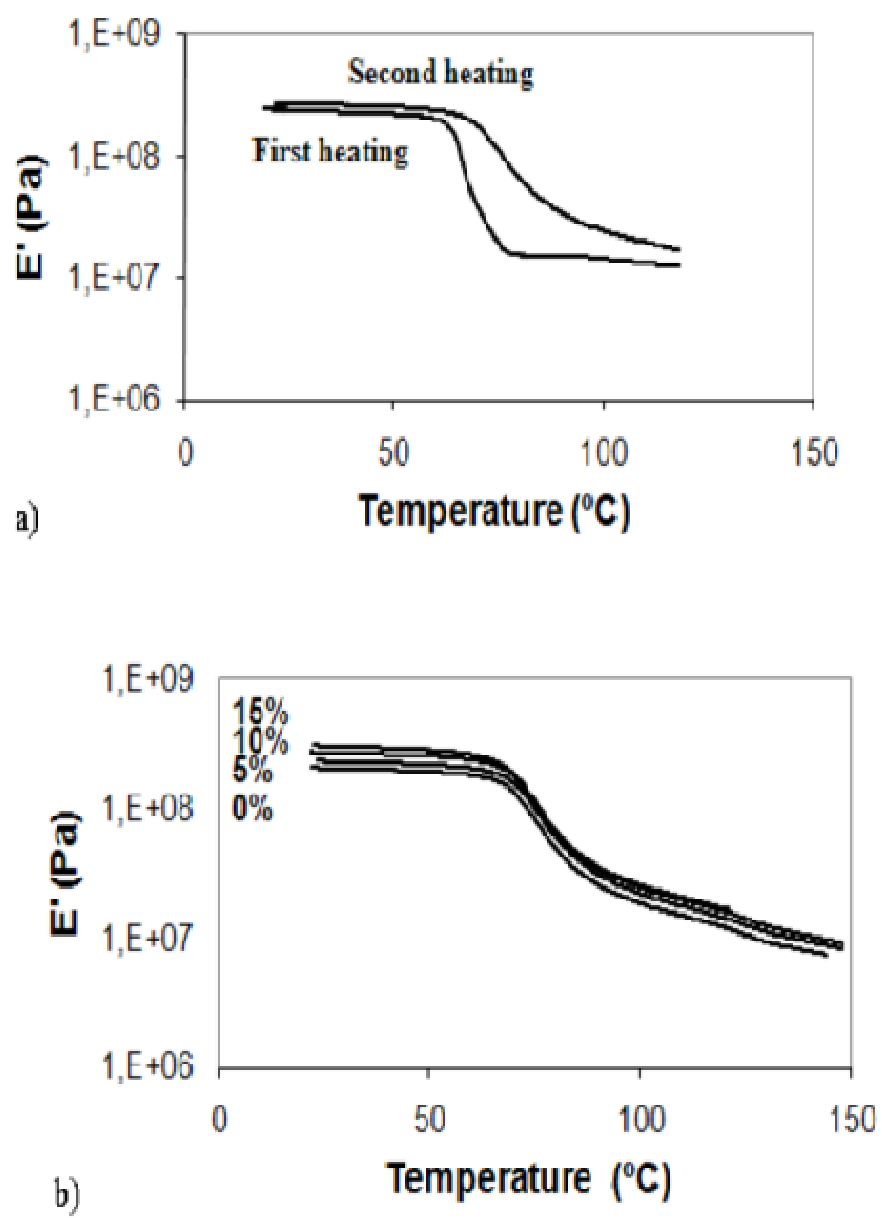

Figure 3-47: Dynamic-mechanical storage modulus as a function of temperature, E'(T), obtained at $1 \mathrm{~Hz}$ and a heating rate of $2^{\circ} \mathrm{C} / \mathrm{min}$ : (a) first and second heating scan of $10 \%$ wt. HAp membrane, (b) second heating scan of $0,5,10$ and $15 \%$ wt. HAp membranes. 
Table 3-11: Dynamic-mechanical storage modulus of the membranes filled with $0,5,10$ and, $15 \%$ wt. of nanoparticles of HAp after the first and the second scan.

\begin{tabular}{cccc}
\hline \multirow{2}{*}{$\begin{array}{c}\text { HAp content } \\
(\%)\end{array}$} & $\mathbf{E}^{\prime}$ glass (Pa) & $\mathbf{E}^{\prime}$ glass (Pa) & $\begin{array}{c}\mathbf{E}^{\prime}(\mathbf{P a}) \\
\text { - after main } \\
\text { relaxation }\end{array}$ \\
\cline { 2 - 4 } & $\begin{array}{c}\left.\mathbf{( 3 0}^{\mathbf{0}} \mathbf{C}\right) \\
\text { first scan }\end{array}$ & $\begin{array}{c}\left.\mathbf{( 3 0}^{\mathbf{o}} \mathbf{C}\right) \\
\text { second scan }\end{array}$ & $\begin{array}{c}\left.\mathbf{( 1 2 0}^{\mathbf{0}} \mathbf{C}\right) \\
\text { second scan }\end{array}$ \\
\hline 0 & $1.81 .10^{8}$ & $2.03 .10^{8}$ & $1.20 .10^{7}$ \\
5 & $2.02 .10^{8}$ & $2.30 .10^{8}$ & $1.43 .10^{7}$ \\
10 & $2.37 .10^{8}$ & $2.68 .10^{8}$ & $1.61 .10^{7}$ \\
15 & $2.56 .10^{8}$ & $3.03 .10^{8}$ & $2.08 .10^{7}$ \\
\hline
\end{tabular}

\subsubsection{Bioactivity}

Figure 3-48 shows the surface of the membranes of PLLA without nanofiller after the maximum time of immersion in SBF (21 days). It is possible to evaluate the influence of the plasma treatment on the bioactivity of the samples over this time. Figure 3-49 shows the surface of the membranes filled with $0,5,10$ and, 15 wt. \% of HAp with and without plasma treatment after 7 days in SBF. In this way it is possible to evaluate the influence of the plasma treatment and the content of nanofiller on the bioactivity of the membranes at short time since the membranes are not affected too much by hydrolysis in SBF.

SEM analysis shows the influence on the kinetics of HAp deposition during increasing immersion times in SBF. As can be seen in Figure 3-48 PLLA is able to nucleate HAp in its surface, without any special treatment but at very slow rates compared to plasma-treated PLLA or PLLA reinforced with HAp. Over short time periods few nuclei can be observed in the SEM pictures, they increase in number to form a rough layer of cauliflower-shaped crystals. The surface of the micropores needs less time of immersion to be uniformly 
coated with HAp as the content of nanofiller increases and with previous exposure to plasma. Figure 3-48 shows the surface of a PLLA membrane after 21 days of immersion in $\mathrm{SBF}$, a time at which coating is still not complete, whereas the sample treated with plasma show a smooth layer of biomimetic HAp deposited on the surface.

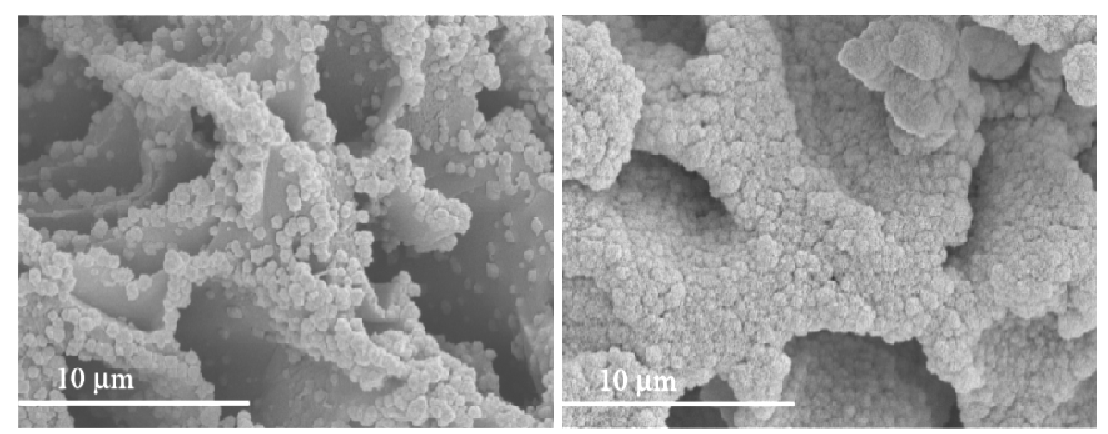

Figure 3-48: Scanning electron micrographs of pure PLLA membranes, without plasma treatment (left) and with oxygen plasma treatment (right), after 21 days in SBF.

Figure 3-49 shows the effect of the HAp nanofiller content after 7 days of immersion. After this time the PLLA samples show no signs of apatite layer even when treated with plasma, but the rate of deposition increases with HAp content and samples containing more than $10 \%$ wt. HAp nano-particles show a smooth coating of the composite surfaces. Plasma treatment further enhances the rate of deposition and the thickness of the layer increases in such a way that biomimetic apatite fills the whole volume of the micropores (Figure 3-49). After 21 days of immersion in SBF as it can be seen in Figure 3-48 a layer of biomimetic HAp covers all the surface of the membranes. As can be seen on Table 3-12 Ca/P atomic ratio of the biomimetic HAp layer has been calculated from EDS analysis. This ratio has been compared to the one of stoichiometric hydroxyapatite $\left(\mathrm{Ca}_{10}\left(\mathrm{PO}_{4}\right)_{6}(\mathrm{OH})_{2}\right) \mathrm{Ca} / \mathrm{P}=1.67$, or physiological HAp $=1.65$. Globally the $\mathrm{Ca} / \mathrm{P}$ atomic ratio 
calculated for all the samples is higher than the physiological HAp one, ranging from 1.63 to 2.54. The membranes with HAp incorporated at day 0 , in which the $\mathrm{Ca} / \mathrm{P}$ atomic ratio measured corresponds to the HAp nanoparticles added as filler, have the highest ratio, and the ratio observed does not match that of stoichiometric hydroxyapatite claimed by the supplier. For the same immersion time membranes previously exposed to plasma present a ratio closer to the physiological one in comparison with membranes that had not been exposed to plasma. In general, the ratio that is closest to the physiological one is for the plasma treated membranes prepared with nano-filler after 7 days in SBF. 


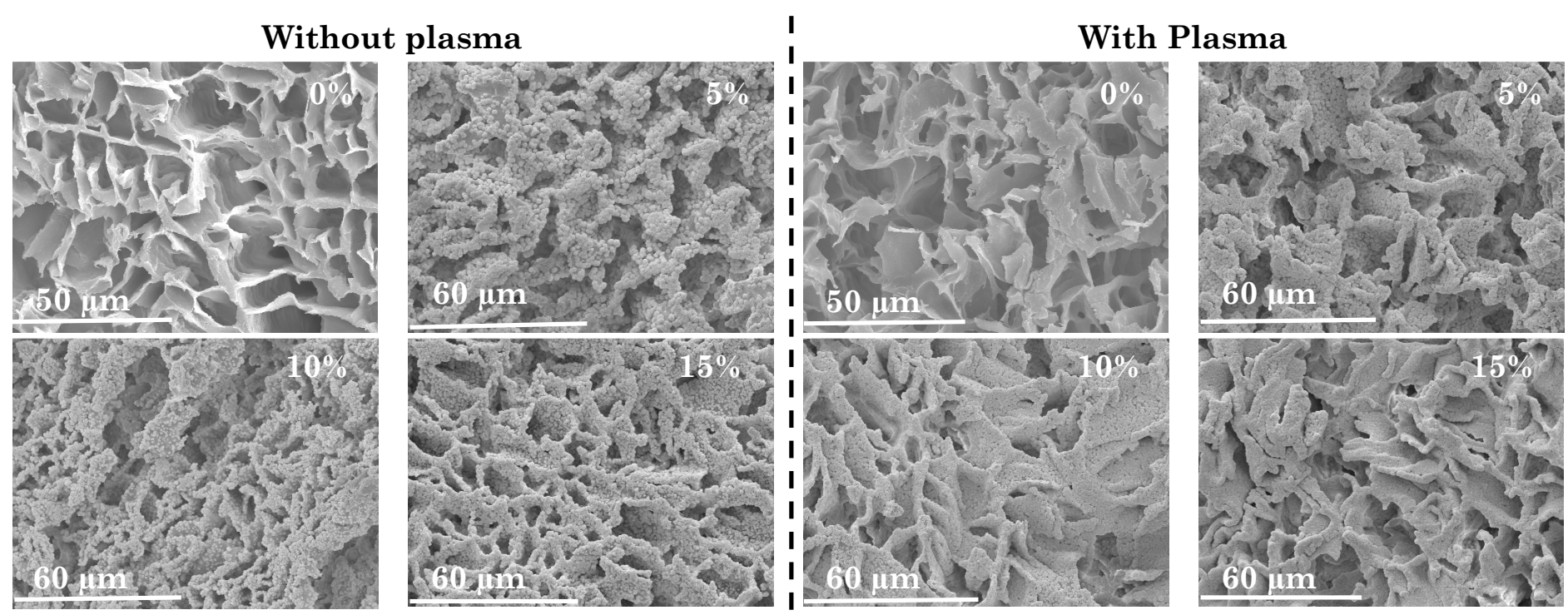

Figure 3-49: Scanning electron micrographs of 0, 5, 10 and $15 \%$ wt. HAp membranes without and with oxygen plasma treatment, after 7 days in SBF. 
Table 3-12: $\mathrm{Ca} / \mathrm{P}$ atomic ratio reported from the energy dispersive spectroscopy analysis

\begin{tabular}{|c|c|c|c|c|c|c|c|c|c|}
\hline \multicolumn{2}{|c|}{ Immersion time (days) } & \multicolumn{2}{|l|}{0} & \multicolumn{2}{|l|}{7} & \multicolumn{2}{|l|}{14} & \multicolumn{2}{|l|}{21} \\
\hline HAp content (\%) & $\begin{array}{l}\text { Plasma } \\
\text { treatment }\end{array}$ & no & yes & no & yes & no & yes & no & yes \\
\hline 0 & & $0 \pm 0$ & $0 \pm 0$ & $0 \pm 0$ & $0 \pm 0$ & - & - & $2.18 \pm 0.6$ & $1.77 \pm 0.4$ \\
\hline 5 & & $2.54 \pm 0.5$ & $2.15 \pm 0.4$ & $1.82 \pm 0.1$ & $1.66 \pm 0.1$ & - & - & $2.55 \pm 0.7$ & $1.77 \pm 0.3$ \\
\hline 10 & & $2.1 \pm 0.2$ & $2.38 \pm 0.4$ & $1.68 \pm 0.05$ & $1.67 \pm 0.05$ & $2.25 \pm 0.7$ & $1.95 \pm 0.2$ & $2.2 \pm 0.3$ & $2.48 \pm 1.3$ \\
\hline 15 & & $2.21 \pm 0.3$ & $2.33 \pm 0.4$ & $1.63 \pm 0.1$ & $1.69 \pm 0.2$ & - & - & $2.08 \pm 0.2$ & $2.62 \pm 1.4$ \\
\hline
\end{tabular}




\subsubsection{D hybrid structure}

The influence of the incorporation of HAp nanoparticles when fabricating 3D scaffolds and the influence of plasma treatment will be now discussed. The material used to fabricate this scaffolds was PLLA for industrial use; PLLA-CD. This was in order to lower the cost of the study as it is a first step in $3 \mathrm{D}$. In this first section the nanofiller used is nanoparticles of HAp.

\subsubsection{PLLA/HAp composites}

\section{MORPHOLOGY}

Figure 3-50 shows the surface of the scaffolds only made with PLLA and those filled with $5 \%$ wt. of HAp. It can be observed that the morphology of the scaffolds is not affected, neither macro nor microstructure show differences when the nanofiller is incorporated to the polymer matrix. Although it will be discussed more thoroughly later in the section on mechanical properties, it is worth mentioning now that the elastic modulus of the scaffolds filled with HAp decreases - see Figure 3-57 and Figure 3-58. The viscosity of the polymeric matrix used to produce composite porous scaffolds of PLLA is one of the main difficulties to permit a uniform distribution of the filler inside the polymeric matrix and avoid aggregates of particles, which also affects the mechanical properties. 


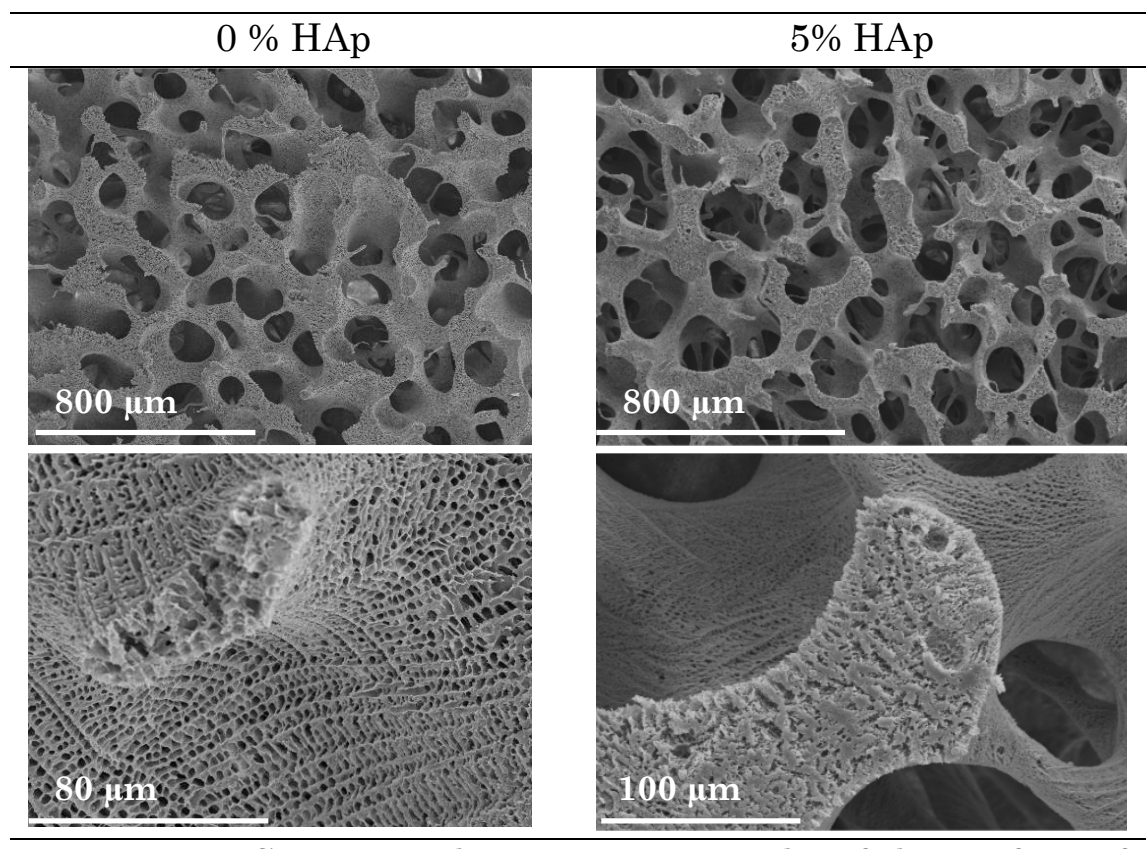

Figure 3-50: Scanning electron micrographs of the surface of the scaffolds of 1:1-(15/85)-CD scaffolds and 1:1-(15/85)-CD scaffolds filled with $5 \%$ wt. of nanoparticles of HAp.

\section{BIOACTIVITY}

In Figure 3-51, Figure 3-52 and Figure 3-53 the surface of the scaffolds filled and not filled with nanoparticles of HAp after 7, 14 and 21 days in SBF are shown. It is possible to evaluate the influence of immersion times and the incorporation of the nanofiller after 21 days in SBF (Figure 3-53) and the influence of plasma treatment after 14 days in SBF (Figure 3-52) on the bioactivity of the scaffolds. Figure 3-54 shows the morphology of the biomimetic HAp coated on the surface of the scaffolds and its distribution in the macropore scale. Figure 3-55 is a micrograph of the scans made during elemental analysis on the surface of the scaffolds filled with $5 \%$ wt. of nanoparticles of HAp and exposed to plasma after 21 days in SBF. It also shows the corresponding scan of the signal obtained during 
the elemental analysis. Table 3-13 compiles the calcium phosphate ratios on the surface of the scaffolds filled with 0 and $5 \%$ wt. HAp nanoparticles and treated or not by plasma treatment after 14 and 21 days in immersion in SBF. Figure 3-56 is a histogram showing the calcium and phosphate ratios in the biomimetic layer coating on the surface of the scaffolds after 21 days (when bioactivity tends to be the most satisfactory. No nuclei of HAp were detected on the surface of the scaffolds after 7 days in SBF. It can be appreciated the influence of the nanofiller and the plasma treatment on the nature of the layer of HAp nucleated on the surface of the samples.

Apatite layer formation in the surface of the pores of the scaffolds is observed after different times of immersion in SBF. Nanoparticles of HAp were added and a previous oxygen plasma treatment applied as a mean for studying their influence on in vitro bioactivity of the samples. The scaffolds were carefully dipped in SBF using vacuum to remove all the air trapped in the pores. SEM analysis in Figure 3-51 and Figure 3-53 shows the progression of the nucleation of HAp on the surface of the scaffold. The layer nucleated on the surface of the scaffolds is denser than the one in the inside of the scaffolds. It was possible to nucleate a uniform HAp layer inside the matrix for scaffolds with 5\% HAp nanoparticles and a previous plasma treatment after 21 days in SBF. Only plasma treatment after 21 days in SBF allows the formation of some nuclei of HAp inside the scaffolds. The scaffold without filler is able to nucleate HAp on its surface after being 21 days in SBF, but not before this time, as observed in Figure 3-51 and Figure 3-53. These kinetics are accelerated when scaffolds without filler particles are plasma treated (Figure 3-52) or when it is mixed with $5 \%$ of HAp nanoparticles. This is because a uniform layer is already nucleated after 14 days in SBF when one of these processes is applied (Figure 3-51). After 14 and 21 days in SBF the layer of HAp precipitated on 
the surface of the sample, is more uniform for scaffold previously treated with plasma than the ones filled with nanoparticles. As can be seen in Figure 3-51 and Figure 3-52, the combination of plasma and nanoparticles as filler, allows the bioactivity of the scaffolds increase. After 14 days in SBF, plasma modified hybrid scaffolds filled with $5 \%$ of nanofiller are able to nucleate a second hydroxyapatite layer on the surface of the scaffolds (Figure 3-51). After 21 days in SBF the nucleation is also produced on the pore walls in the inside of the scaffolds (Figure 3-52).

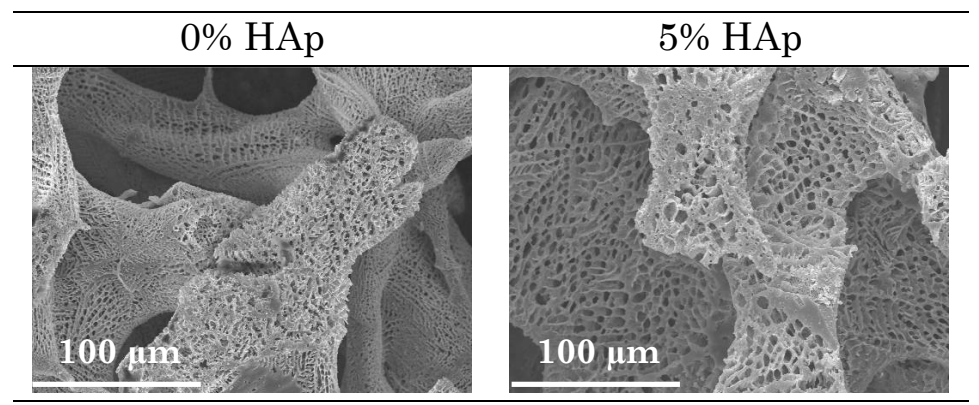

Figure 3-51: Scanning electron micrographs of the surface of the scaffolds filled and not filled with nanoparticles of HAp after 7 days in SBF. 


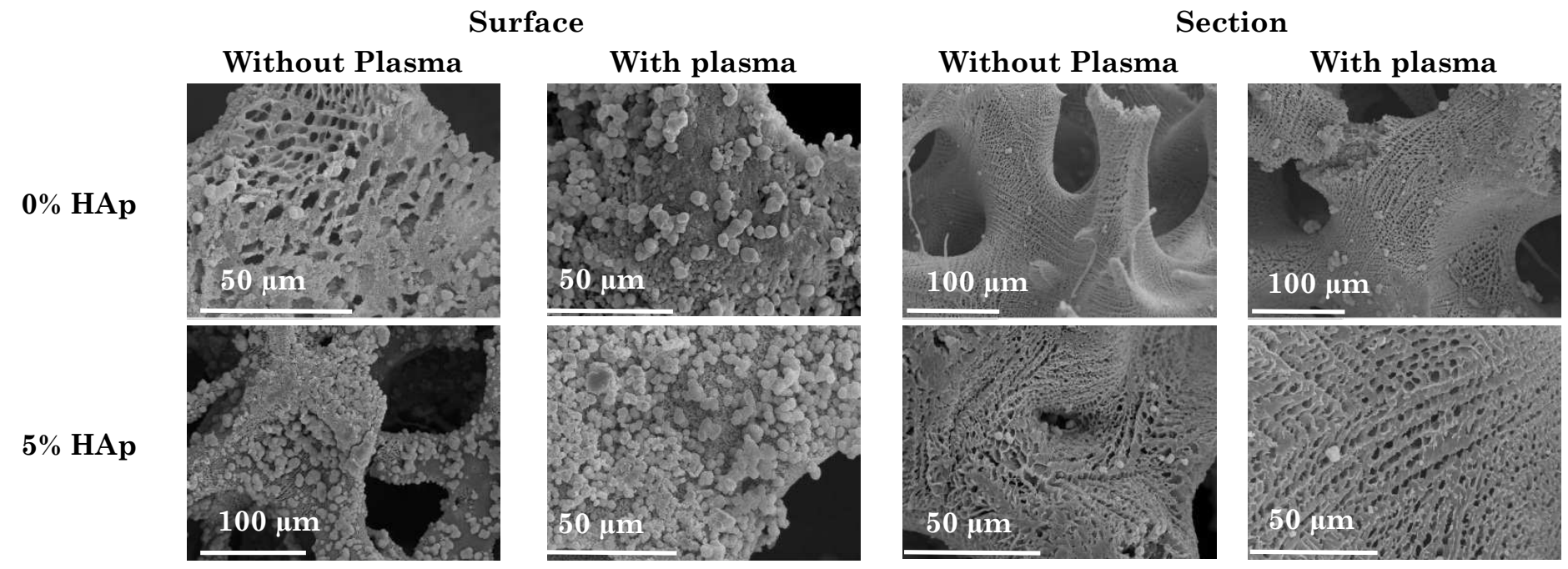

Figure 3-52: Scanning electron micrograph of the surface and the section of the scaffolds of PLLA-CD filled with 0 and $5 \%$ wt. of nanoparticles of HAp after 14 days previously exposed and not exposed to plasma. 
Surface

14 days

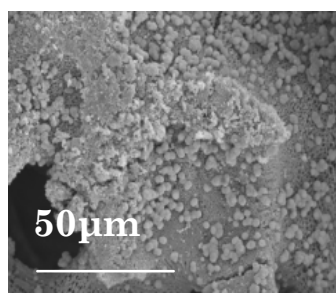

0 \% HAp

5 \% HAp

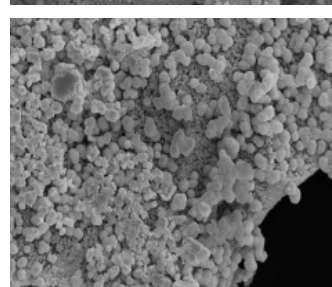

Figure 3-53: SEM micrographs of the surface and section of hybrid scaffolds of PLLA-CD filled with 0 and $5 \%$ wt. of nanoparticles of HAp after 14 and 21 days of immersion in SBF and previous plasma treatment.

Section
21 days

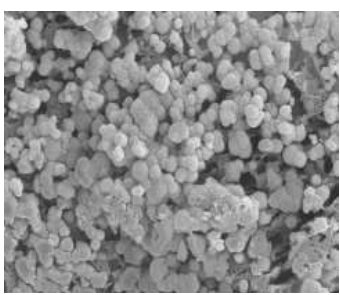

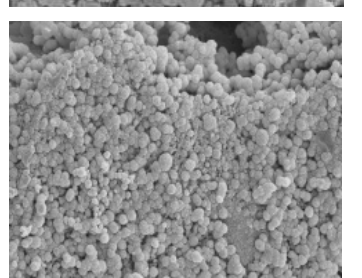
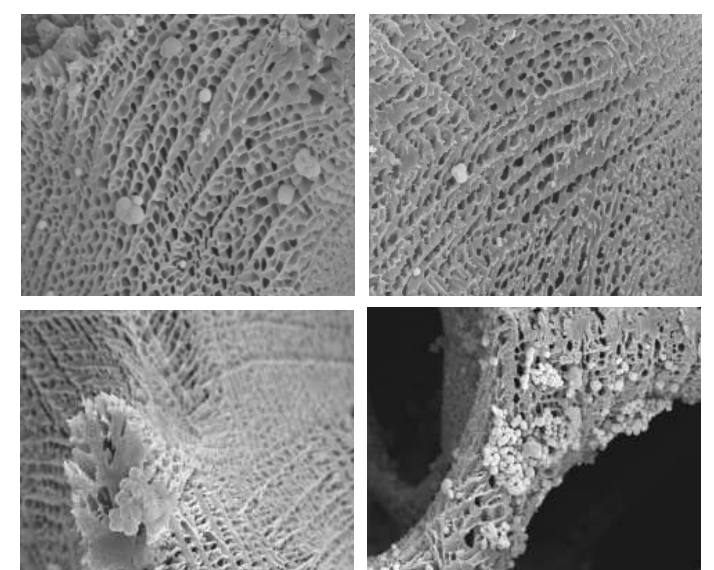
The layer of HAp coated on the scaffold matrix presents the typical cauliflower shape, like hemispherical-shaped apatite granules characteristic to biomimetic HAp from SBF ions precipitation. The granules contain numerous nano-sized apatite needle - like crystals (Figure 3-54) (156). The nucleation of HAp on the surface of the scaffold does not obstruct the macrostructure.
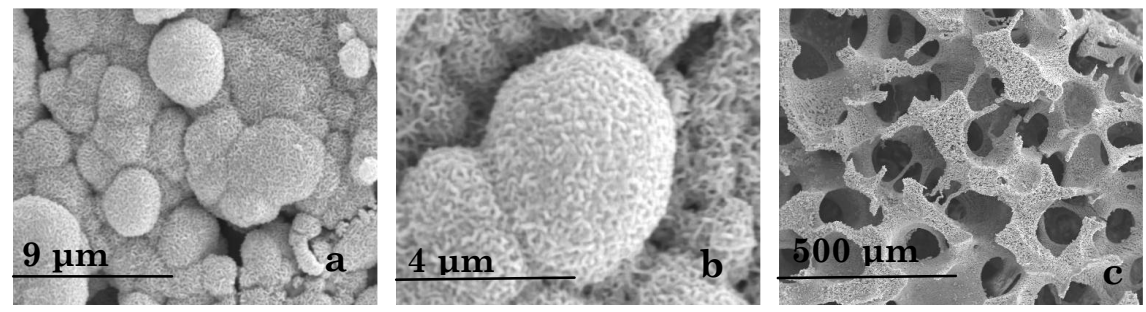

Figure 3-54: SEM micrographs of a) HAp coating on the surface of the scaffolds filled with $5 \%$ wt. of nanoparticles after 14 days in SBF b) same surface but at higher magnification, c) macrostructure of the scaffolds filled with $5 \%$ of nanoparticles after 21 days in SBF.

The $\mathrm{Ca} / \mathrm{P}$ this ratio also was calculated using the EDS signals corresponding to $\mathrm{Ca}$ and $\mathrm{P}$ elements; the spectra of 3 replicates of each sample in 3 different zones were employed and the mean value was calculated. Figure 3-55 corroborates that the EDS detected peaks of the nucleated HAp correspond mainly to calcium and phosphate; the spectra show other peaks corresponding to sodium and chloride, but their signals are not significant enough to be considered. Figure 3-56 shows the $\mathrm{Ca} / \mathrm{P}$ ratio of the hydroxyapatite nucleated on the surface and section of pure PLLA scaffold and PLLA/HAp hybrid scaffold, with and without a previous plasma treatment after 14 and 21 days in SBF. Scaffolds with previous plasma treatment are named (PLLA-P), these filled with $5 \%$ wt. HAp nanoparticles are named (PLLA-5\%), and these filled with 5\% wt. HAp nanoparticles exposed to previous plasma treatment are 
named (PLLA-5\%-P). As shown in Table $3-13$, the $\mathrm{Ca} / \mathrm{P}$ ratio oscillates between 3.23 and 1.52 for the scaffolds immersed in SBF 14 days and between 1.58 and 3.98 for those in SBF during 21 days, for all the scaffolds (plasma/no-plasma, 0 and $5 \%$ wt. of nanofiller). In the section of the scaffolds it can be observed that $\mathrm{Ca} / \mathrm{P}$ is nearer to physiological one after 14 and 21days in SBF for the scaffolds without filler and previously exposed to plasma treatment. But those filled with $5 \%$ of nanofiller present a $\mathrm{Ca} / \mathrm{P}$ nearer to the physiological one when they were not exposed to plasma. In the surface of the scaffolds the $\mathrm{Ca} / \mathrm{P}$ nearer to the physiological one is for the scaffolds after 14 days in SBF filled with $5 \%$ of nanofiller and without plasma exposure, but after 21 days it is for the scaffolds filled with 0 and $5 \%$ wt. of HAp, both exposed to plasma. In some cases, as after 14 days in SBF, little biomimetic HAp is nucleated on the section of the scaffolds, but the calcium phosphate ratio can be close the physiological one (Figure 3-52 and Figure 3-53). These values are highly accurate, demonstrated by an error less than 0.4. Globally the $\mathrm{Ca} / \mathrm{P}$ ratio is nearer to that of the physiological HAp for the scaffolds immersed during 21 days in SBF.
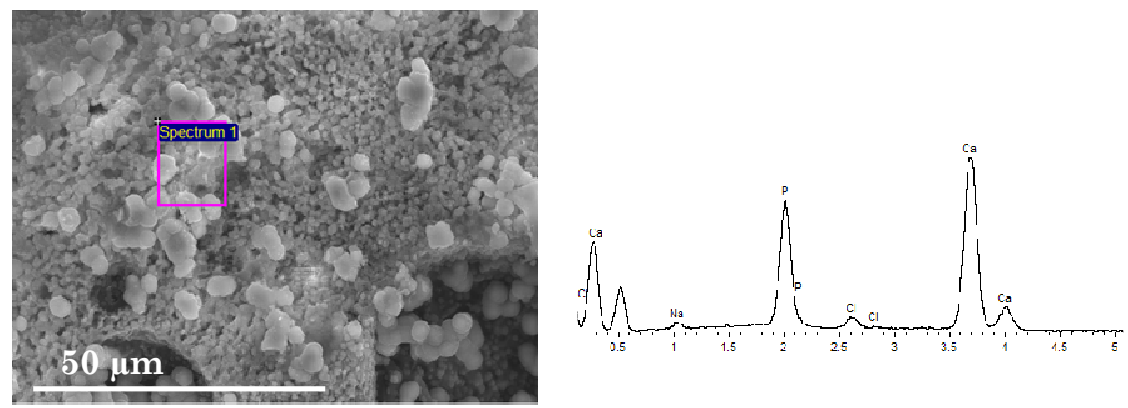

Figure 3-55: On the left Scanning electron micrograph from the elemental analysis of the surface of a scaffold filled with nanoparticles of HAp exposed to plasma and immersed for 21 days in SBF. On the right the corresponding scan of the signal of the elemental analysis. 
Table 3-13 Ca/P ratio after 14 and 21 days in SBF calculated for the section and the surface of scaffolds of PLLA-CD filled with 0 and $5 \%$ wt. of nanoparticles of HAp. These ratios have been calculated from the EDS signal of analysis on the surface and cross-section of the samples.

\begin{tabular}{|c|c|c|c|c|}
\hline & & & 14 days & 21 days \\
\hline \multirow{4}{*}{ section } & \multirow{2}{*}{$0 \%$ HAp } & No Plasma & $3,23 \pm 3,4$ & $2,3 \pm 1,2$ \\
\hline & & Plasma & $1,81 \pm 0,4$ & $1,58 \pm 0,1$ \\
\hline & \multirow{2}{*}{$5 \%$ HAp } & No Plasma & $1,52 \pm 0,1$ & $1,70 \pm 0,2$ \\
\hline & & Plasma & $2,95 \pm 2,7$ & $2,39 \pm 1,6$ \\
\hline \multirow{4}{*}{ surface } & \multirow{2}{*}{$0 \%$ HAp } & No Plasma & $2,28 \pm 1,7$ & $3,98 \pm 3,2$ \\
\hline & & Plasma & $2,63 \pm 1,5$ & $1,67 \pm 0,2$ \\
\hline & \multirow{2}{*}{$5 \%$ HAp } & No Plasma & $1,63 \pm 0,1$ & $1,92 \pm 0,4$ \\
\hline & & Plasma & $2,69 \pm 2,6$ & $1,59 \pm 0,1$ \\
\hline
\end{tabular}

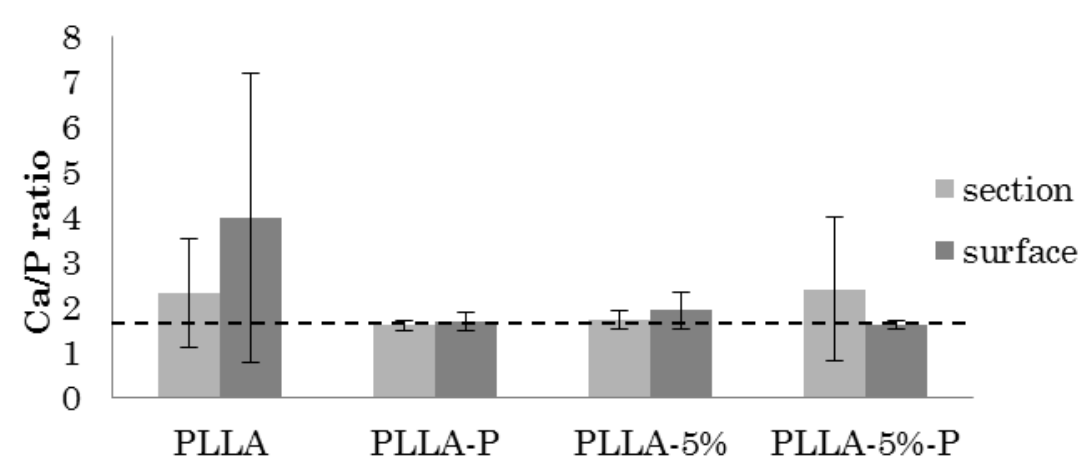

Figure 3-56: $\mathrm{Ca} / \mathrm{P}$ ratio after 21 days in SBF calculated for the cross-section and the surface of scaffolds of PLLA-CD filled with 0 and $5 \%$ wt. of nanoparticles of HAp. These ratios have been calculated from the EDS signals of analysis on the surface and section of the samples. 


\section{MECHANICAL PROPERTIES}

The apparent Young's modulus measured in compression mode was obtained from 5 measurements of different scaffolds with the same dimensions under the same conditions. In Figure 3-57 the stress vs. strain curves resulting from the compression test is shown. The elastic modulus was calculated for the elastic zone. In Figure 3-58 the various moduli obtained are compared. From the curves it can be observed that the elastic zone is clearly reducing towards lower stress and strain after 14 and 21 days in SBF independently from the content of filler These measurements show that the elastic modulus decreases from $8.9 \pm 0.4 \mathrm{MPa}$ for the original pure PLLA scaffold to $4.5 \pm 1.1 \mathrm{MPa}$ for the one filled with nanoparticles. Figure 3-58 also shows that the modulus is also decreasing with the time of immersion in SBF. However, it can be seen that the modulus is not decreasing as much for the scaffold composites (PLLA-5\%) after 14 and 21 days in SBF in comparison to those without filler. Indeed, the modulus of the composite is $0.9 \mathrm{MPa}$ higher than that of the pure PLLA scaffold both after immersion in SBF for 21 days, when the bioactivity is supposed to be the highest. That difference is lower at 14 days of immersion, $0.2 \mathrm{MPa}$ difference. The possible hydrolysis degradation of polymer chains in SBF could explain the decrease of mechanical properties of the scaffolds after immersion in SBF. 


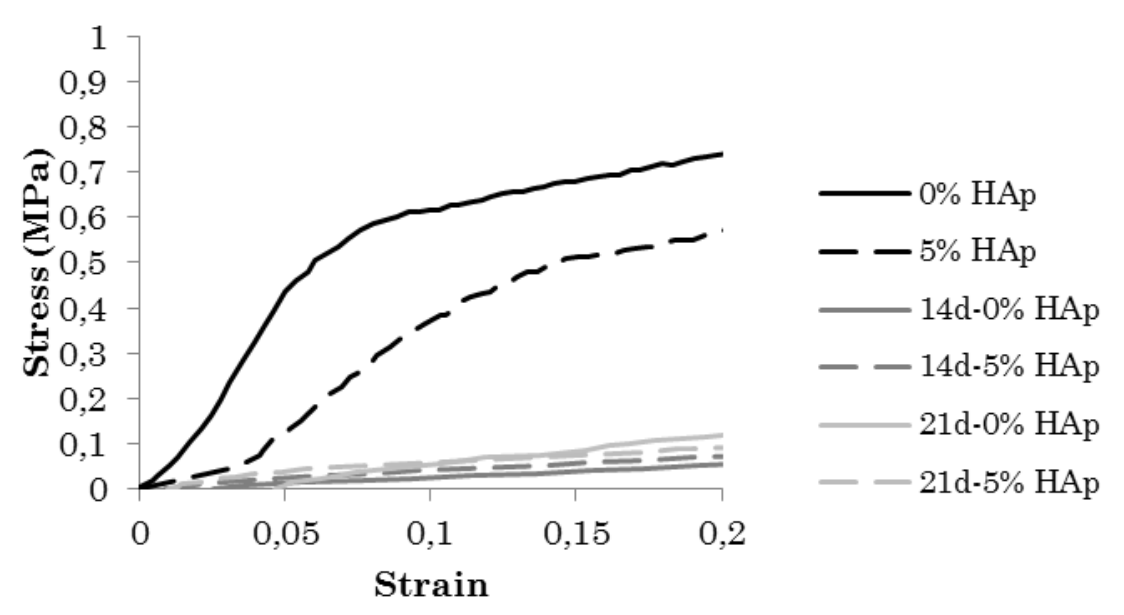

Figure 3-57: Stress and strain representation of the scaffolds filled with $5 \%$ of HAp and not filled after 0,14 and 21 days in SBF. Graph obtained from compression test.

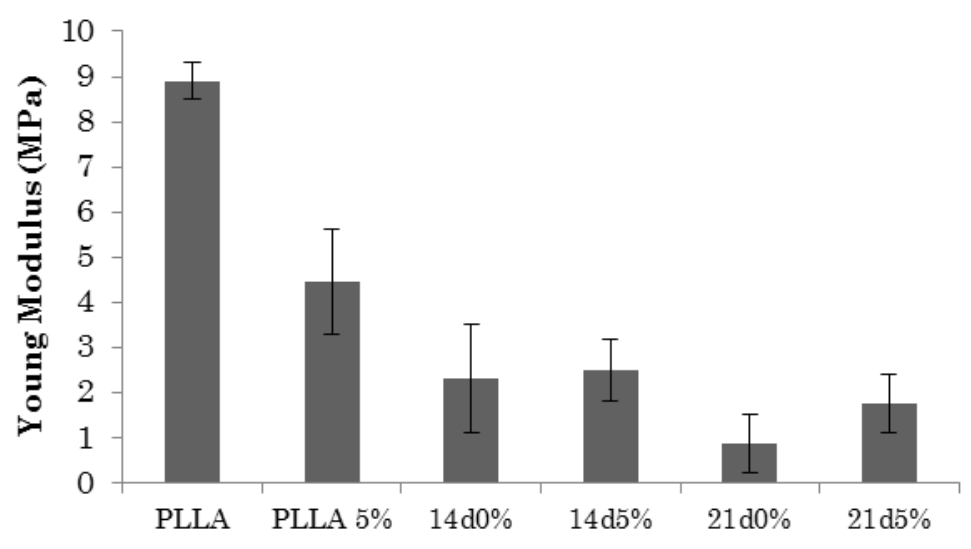

Figure 3-58: Young's modulus obtained from the compressive curves of the scaffolds filled with 0 and $5 \%$ wt. of nanoparticles of HAp after 0/14 and 21 days in SBF. 


\section{GEL PERMEATION CHROMATOGRAPHY}

Figure 3-59 and Figure 3-60 show the molecular weight and the polydispersity index of the scaffolds not filled and filled with nanoparticles of HAp after 0, 14 and 21 days in SBF, with and without plasma treatment. The molecular weight decreases with immersion time, in all cases. These results are consistent with the above mentioned decrease of the mechanical properties with the time of immersion in SBF, the cause of which being the degradation of the polymer matrix. After 14 days the molecular weight decrease is higher for the scaffolds previously exposed to plasma, decreasing from 237 to $190 \mathrm{kDa}$. After 21 days the scaffolds presenting less decrease in molecular weight are those filled with nanoparticles. The smaller molecular weight observed after 21 days of immersion is for the scaffolds made with just PLLA; $66 \mathrm{kDa}$, which is similar to the molecular weight observed for the same scaffolds after 1 year of static degradation in PBS (see the results in section 3.2.1.6, p.97). The polydispersity index increases after 14 days in $\mathrm{SBF}$, except for the scaffolds filled and exposed to plasma. After 21 days the polydispersity index decreased to values smaller than the scaffolds not immersed. The possible hydrolysis degradation of the polymer in SBF could cause the scission of the polymer chains. As PLLA is hydrophobic the scission of the polymer chains is not uniform. This can explain the increase of the polydispersity index with time of immersion. 


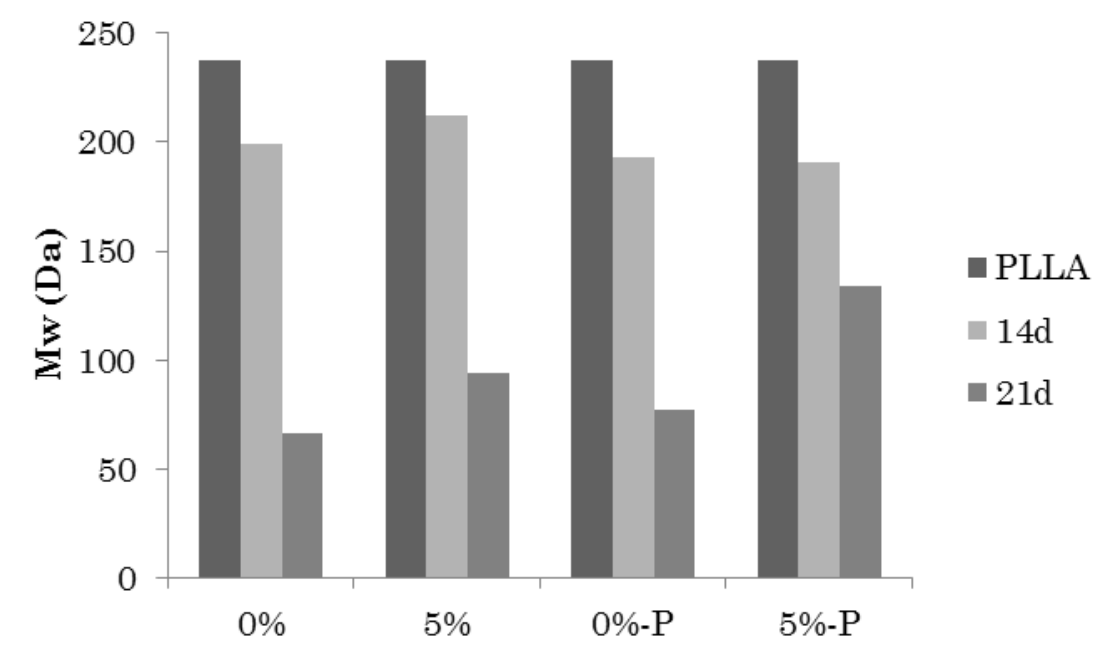

Figure 3-59: Weight average molecular weight of the scaffolds filled with 0 and $5 \%$ wt. of nanofiller previously exposed (P) or not to plasma after 0, 14 and, 21 days in SBF, obtained by the integration of the curves from the GPC test.

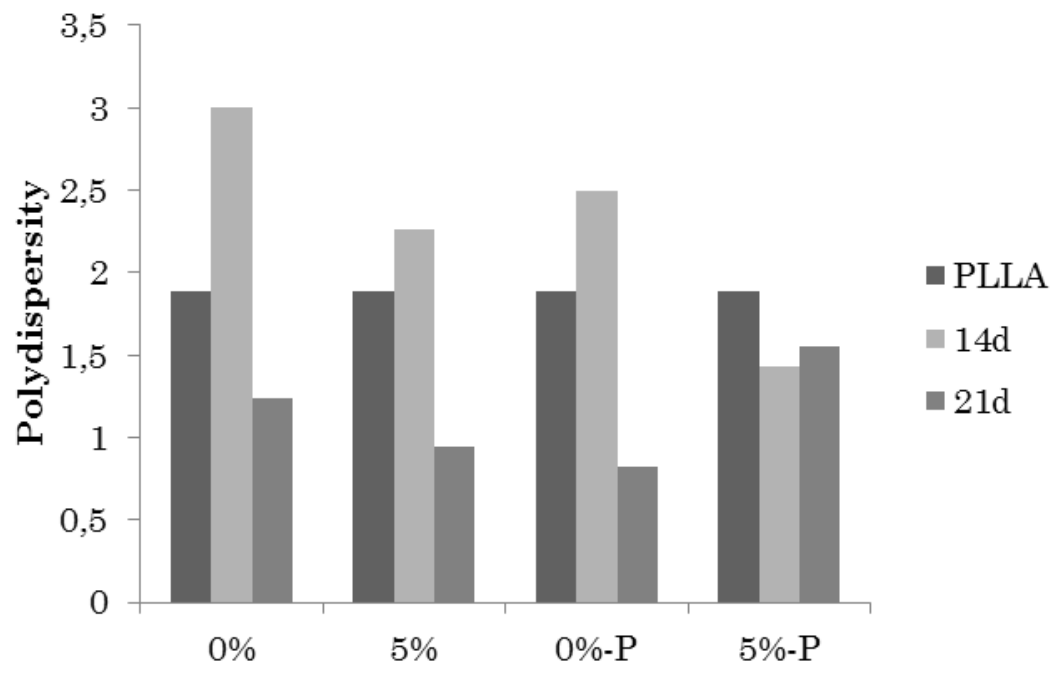

Figure 3-60: Polydispersity index of the scaffolds filled with 0 and $5 \mathrm{wt} \%$ of nanofiller previously exposed (P) or not to plasma after 0,14 and, 21 days in SBF, obtained by the integration of the curves from the GPC test. 


\section{POROSITY}

The influence on the porosity of the HAp layer nucleated in the scaffolds when immersed in SBF was evaluated; the results of the test are compiled in Figure 3-61. The measurements were repeated 3 times for each sample. The global porosity meaning micro and macro-porosity calculated according to equation (5) seems to be not affected by the HAp layer, even when the samples were pre-treated with plasma or charged with nanoparticles. The porosity is still high, about $90 \%$. These results were as expected as the inside of the scaffolds do not present a thick layer of HAp coating. Despite after 21 days in SBF the inside of the scaffolds contain some HAp crystals, the micropores are still clearly observed by SEM, Figure 3-53, and those crystals do not have an influence on the measured porosity.

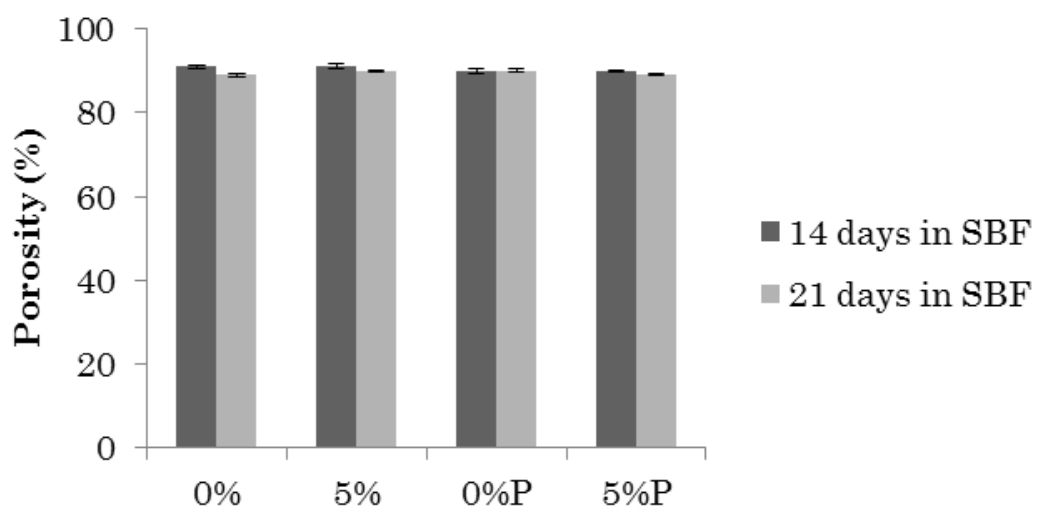

Figure 3-61: Porosity of the scaffolds filled with 0/5\% of nanofiller previously exposed (P) or not to plasma after 14 and 21 days in SBF. 


\section{MASS OF HYDROXYAPATITE COATING}

Figure 3-62, Figure 3-63 and Figure 3-64 show the TGA results for the hybrids scaffolds after 0,14 and 21 days in SBF. This way it is possible to evaluate the influence of the incorporation of the filler or of the plasma treatment on the quantity of biomimetic HAp coated on the surface of the scaffolds. In Figure 3-65 histograms present the percentage of the biomimetic HAp coating on the surface of the scaffolds after 14 and 21 days. The quantity of hydroxyapatite coating the surface of the pores samples was evaluated from the weight percentage representation. The residual percentage of inorganic component obtained after thermal degradation to $1000^{\circ} \mathrm{C}$ corresponds to the total percentage of HAp present, including the percentage nanofiller incorporated and the percentage of HAp coating on the surface of the scaffolds. To obtain the content of biomimetic HAp coated it is necessary to rest the content of nanofiller incorporated during fabrication process. It is observed that the layer of HAp is getting thicker with time since the percentage of residue increases. The higher amount of HAp nucleated on the surface of the scaffolds, almost $23 \%$ of the mass of the sample, is observed for the scaffolds filled with nanoparticles of HAp and previously exposed to plasma treatment. Only by filling the scaffolds with nanoparticles it is possible to nucleate around $13 \%$ of HAp on their surface. 


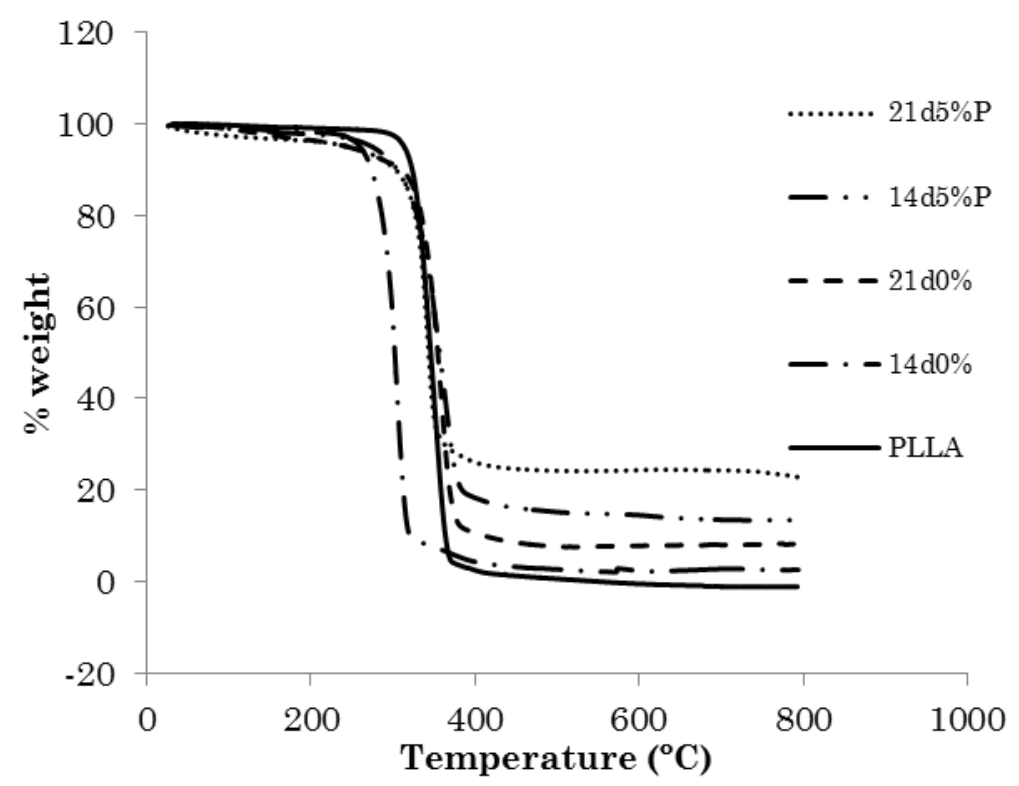

Figure 3-62: Weight percentage as a function of the temperature obtained from TGA test of the scaffolds filled with 0 and $5 \mathrm{wt} \%$ of nanofiller and the scaffolds filled with $5 \%$ of nanofiller and previously exposed to plasma after 0,14 and, 21 days in SBF. 


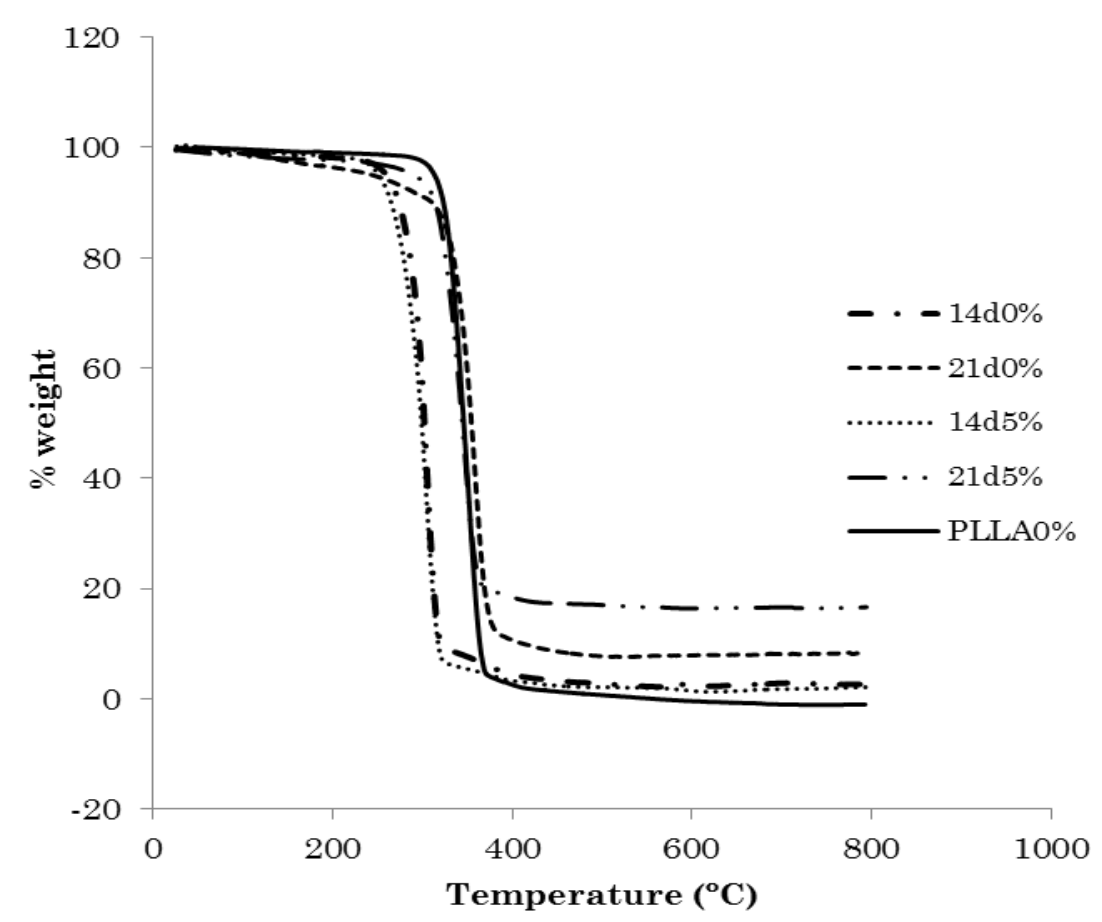

Figure 3-63: Weight percentage as a function of the temperature obtained from TGA test of the scaffolds filled with 0 and $5 \%$ wt. of nanofiller after 0,14 and, 21 days in SBF. 


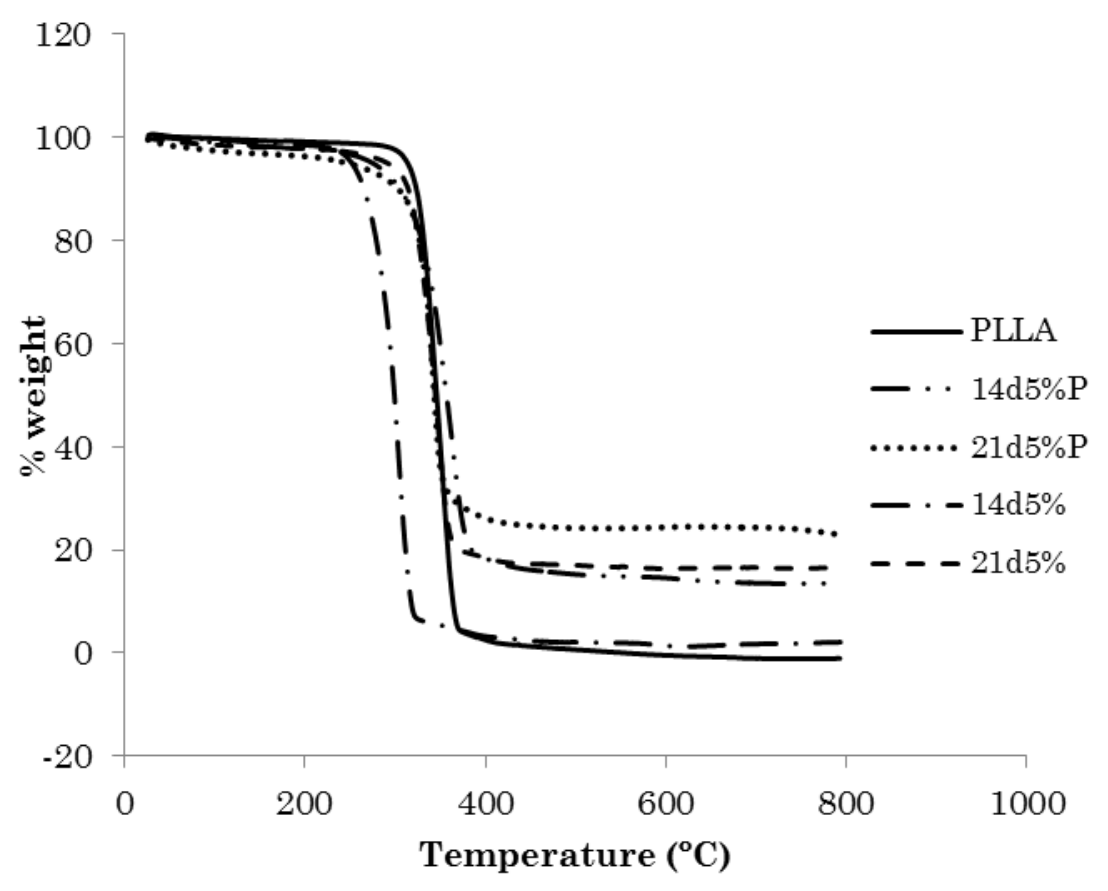

Figure 3-64: Weight percentage as a function of the temperature obtained from TGA test of the scaffolds filled with $5 \%$ of nanofiller previously exposed to plasma (P) or not after 0,14 and, 21 days in SBF. 


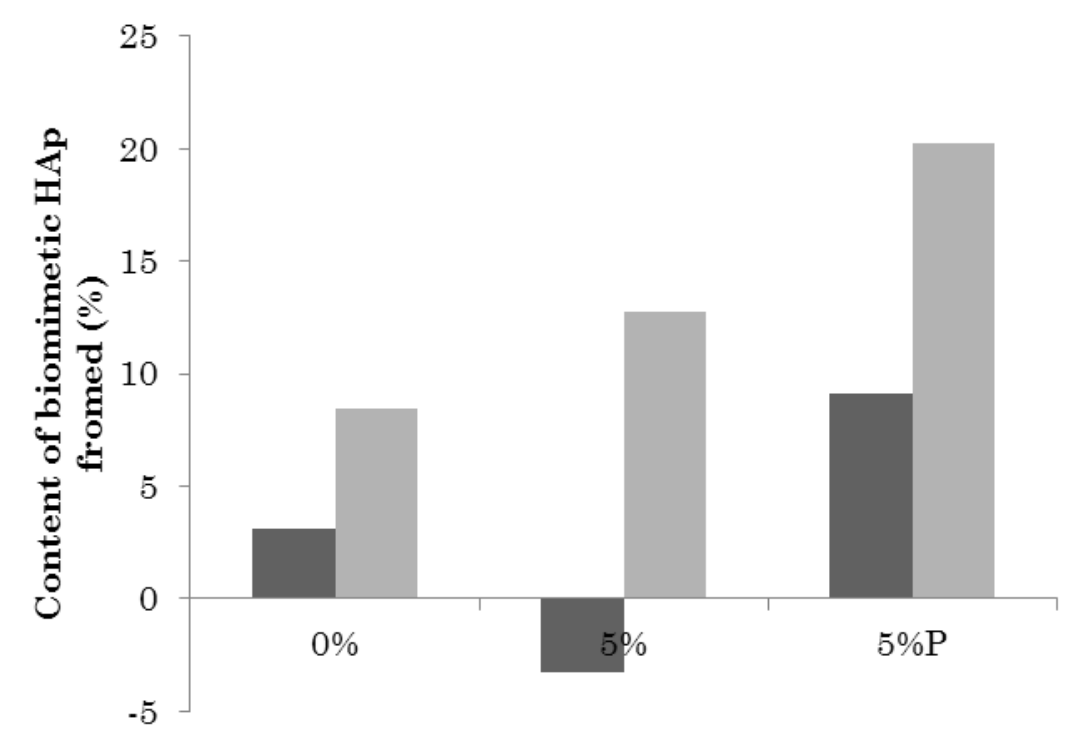

Figure 3-65: Percentage of biomimetic HAp formed on the surface of the scaffolds filled with 0 and $5 \%$ wt. and the scaffolds filled with $5 \%$ of nanofiller and previously exposed to plasma $(P)$ obtained from the TGA residue curves.

\section{FOURIER TRANSFORM INFRARED ANALYSIS}

The FTIR curves of Figure 3-66 and Figure 3-67 are shown in order to compare the influences of the immersion time and the influence of the incorporation of nanoparticles on the characteristics of the HAp coating on the surface of the scaffolds when immersed in SBF. The chemical groups corresponding to each detected stretching band are detailed on the figures. The curves of the commercial HAp nanopowder incorporated in the scaffolds as nanofiller can be used as reference curves to analyse the nature of the HAp coating on the surface of the scaffolds. The main peaks representative of HAp appear in all scaffolds, indicating that all of them can effectively nucleate some hydroxyapatite. It can be seen that 
the main peaks of the nanoparticles from the HAp powder are the same as those obtained for the scaffolds after their immersion in SBF. Furthermore the transmittance peaks are more pronounced for the scaffolds filled with nanoparticles (Figure 3-67) than those without filler (Figure 3-66), probably indicating that the nucleation is more effective in the hybrids than in pure PLLA.

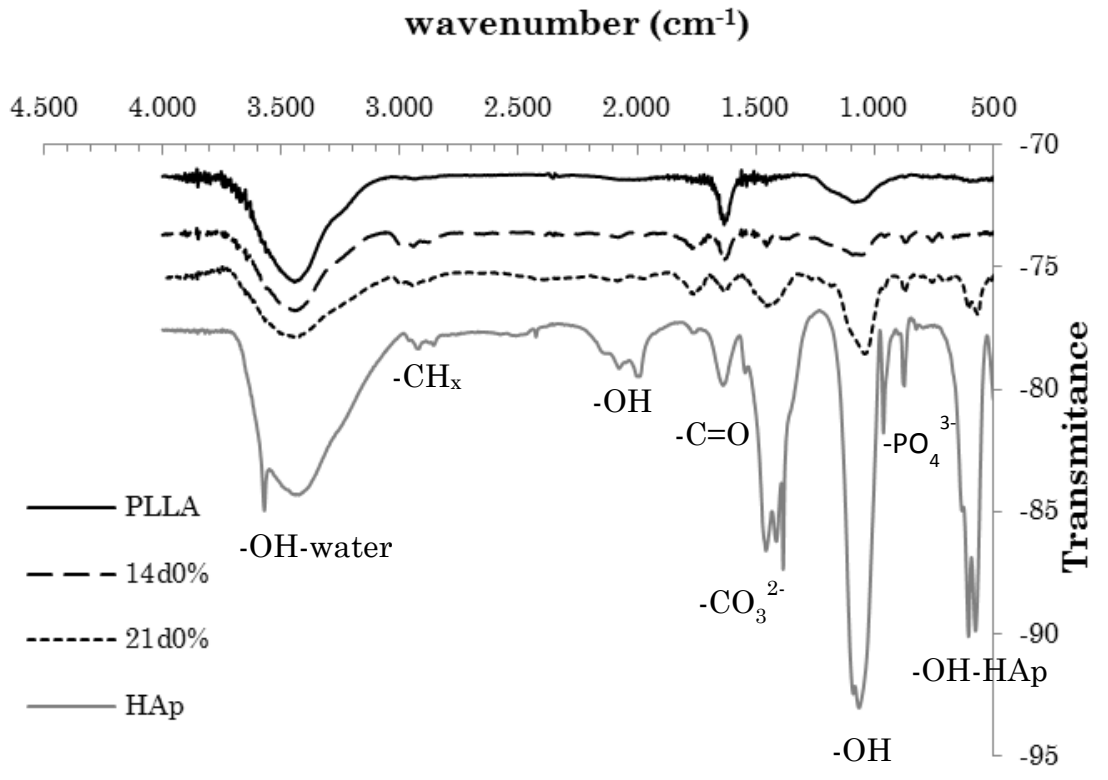

Figure 3-66: Wavenumber vs. transmittance representation obtained from the FTIR analysis of the scaffolds without filler after 0, 14 and, 21 days in SBF, and of HAp nanopowder. 


\section{wavenumber $\left(\mathrm{cm}^{-1}\right)$}

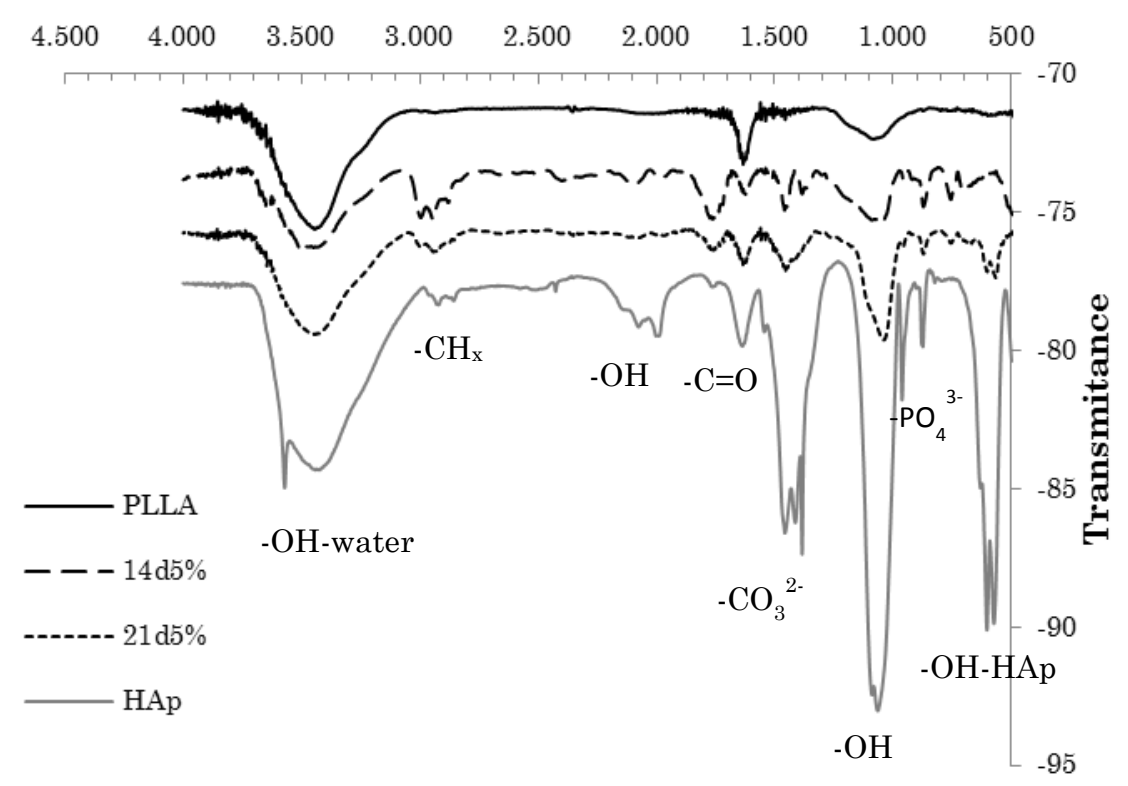

Figure 3-67: Wavenumber vs. transmittance graph obtained from the FTIR analysis of the scaffolds filled with $5 \%$ of HAp particles after 0,14 and, 21 days in SBF, and of HAp nanopowder.

3.3.2.2 Improvements in the mechanical properties of the PLLA/HAp hybrid scaffolds.

In the previous section PLLA was mixed with a 5\% wt. of HAp particles for two purposes: to increase the bioactivity of the scaffolds and to improve their Young's modulus at compression. This was in order to better approximate the properties of natural bone. Although the nanoparticles have increased the bioactivity of the materials, the mechanical modulus has been greatly reduced. There are two reasons for this. Firstly, inorganic phase content may not have been sufficient to form a continuous phase in the organic matrix, and therefore does not provide mechanical reinforcement. 
Secondly, poor dispersion of the nanoparticles would produce agglomeration of large particles.

Two different experiments were designed in order to improve the mechanical properties of the scaffolds. First, the content of nanoparticles was increased and second the sonication procedure, normally applied to disperse the nanoparticles (one sonication when mixing with the dioxane), was duplicated and applied again after the complete dissolution of the polymer in the dioxane/nanoparticles mixture.

\section{INCREASE OF THE HAP NANOPARTICLE CONTENT}

In this part of the study PLLA-MED was used instead of PLLA-CD, which was used up until this point. As the final scaffolds will be fabricated with medical grade PLLA, the reinforcement of the nanofiller on the scaffolds made with PLLA-MED was studied.

Figure 3-68 and Figure 3-69 show the stress and strain curves from the compression test. Figure 3-70 shows the values of the apparent Young's modulus in compression of the different samples calculated from the elastic zone, identified by their HAp content. It can be seen that the elastic modulus of the scaffolds remains almost constant with the increase of the HAp content. Even when PLLA was mixed with a $20 \%$ wt. of nanoparticles it did not increase in comparison to pure PLLA. The stress and strain graphs follow a similar trend, showing little influence from the content in nanofiller incorporated to the polymeric matrix. The measures were repeated for 5 samples of each composition. Since previously it was seen that storage modulus of the hydrid membranes increased with the incorporation of nanofiller, the same result was expected for the hybrid scaffolds. Unfortunately this was not the case. 


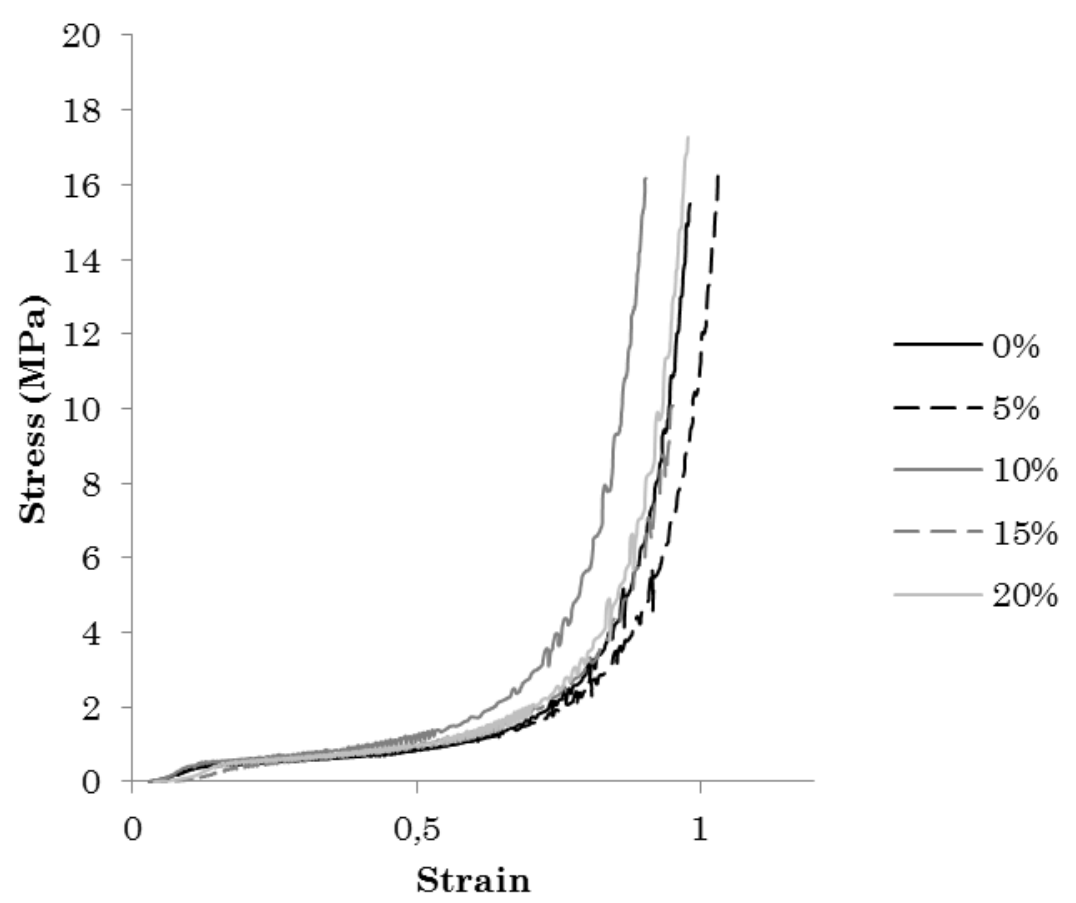

Figure 3-68: Stress vs. strain curves of the scaffolds filled with $0,5,10,15$ and, $20 \%$ wt. of HAp. Representation obtained from compression test. 


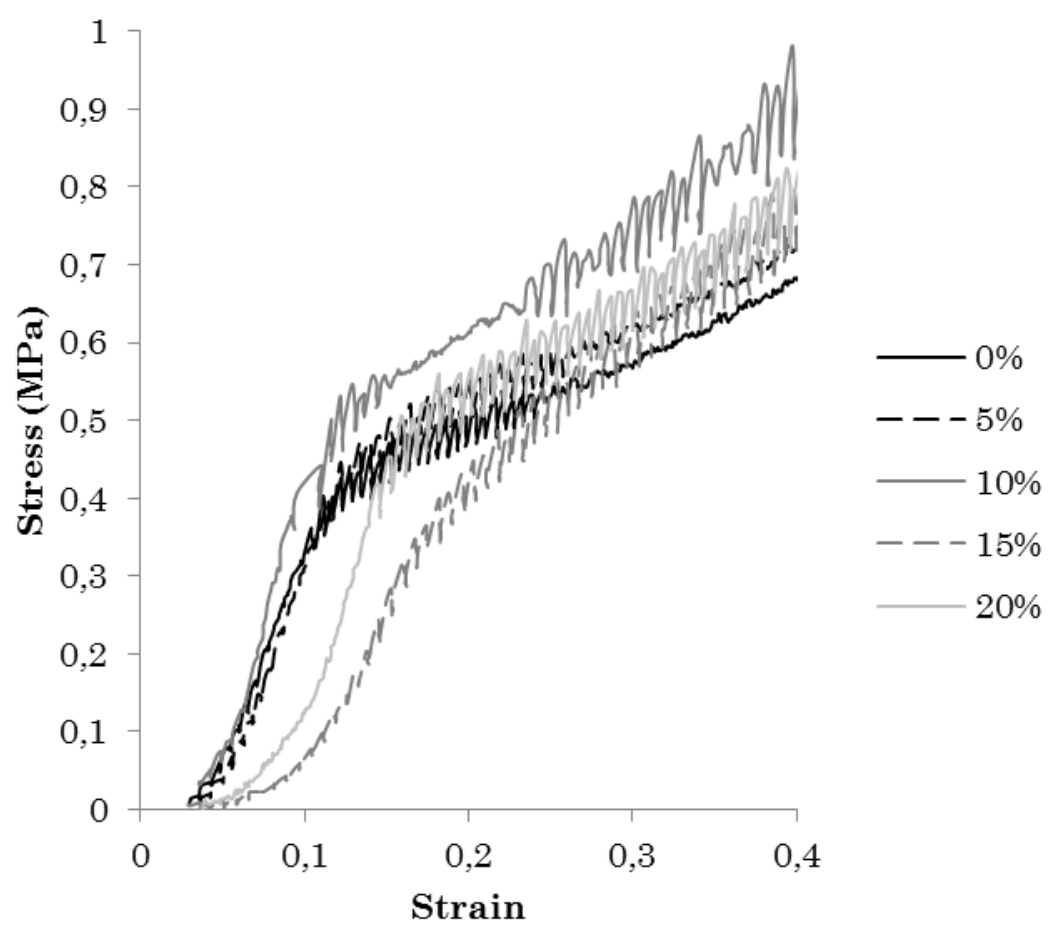

Figure 3-69: Stress vs. strain curves focused on the elastic zone of the scaffolds filled with $0,5,10,15$ and, $20 \%$ wt. of HAp. Graph obtained from compression test.

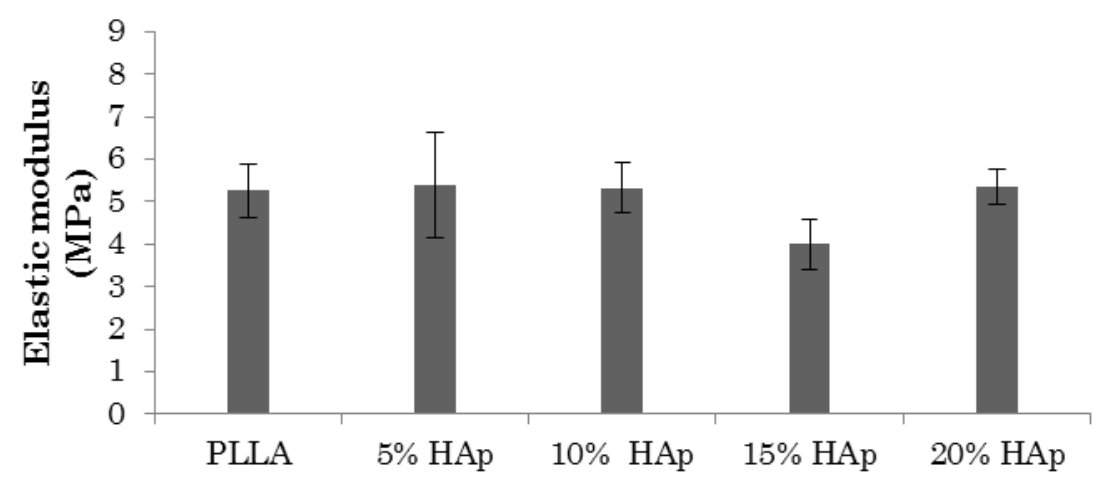

Figure 3-70: Apparent elastic modulus of compression of the scaffolds filled with $0,5,10,15$ and $20 \%$ wt. of HAp nanoparticles. 
The lack of improvement of the mechanical properties of the scaffolds when they are filled with particles of HAp, may be due to a weak dispersion of the particles in the matrix. Sonication is used in order to enhance the nanoparticles dispersion and consequently increase the mechanical strength. Figure 3-71 shows the influence of a double sonication on the mechanical properties of the scaffolds. Unfortunately the scaffolds filled with 5 and $10 \%$ wt. of HAp particles do not show any increase in the elastic modulus.

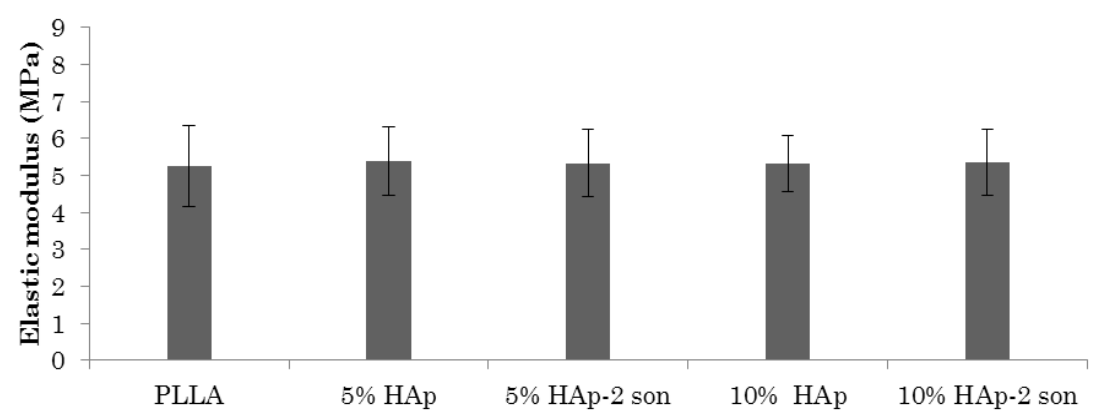

Figure 3-71: Values of the elastic modulus of compression of the scaffolds filled with $0,5,10$ and, $15 \%$ wt. of nanoparticles of HAp after one sonication cycle (5\% HAp and 10\% HAp) and two sonication cycles (5\% HAp-2 son and 10\% HAp-2son).

The hybrid composites made from PLLA and nanoparticles of HAp presented in this section were bioactive. But, as shown their mechanical properties did not improve compared to pure PLLA. One of the major challenges in the design of these scaffolds is that they should retain the ability to sustain loads while tissue regenerates. In order to enhance the mechanical strength of the scaffold and maintain or even improve the material's bioactivity, we changed the filler and used finer bioactive particles based on silica, alone or in combination with the commercial HAp ones used in the previous experiments. 
Five types of hybrid scaffolds were fabricated with different filler contents. The different scaffolds will be named as (100/0/0), (95/0/5), (90/10/0), (90/9.5/0.5) corresponding to the (PLLA/HAp/SiO $/ \mathrm{Si}_{2}$ content. The influence of different nanofiller contents and its effect on the properties of the scaffolds and its bioactivity in SBF during 7, 14 and 21 days was studied. The effect of sonication was also studied in order to improve the dispersion of the filler and therefore the mechanical properties.

\subsubsection{PLLA/HAp/SiO 2 composites}

\section{SONICATION TO DISPERSE HAP / $\mathrm{SIO}_{2}$ PARTICLES}

In order to increase the surface of interaction between the filler and the polymeric matrix, the effect of sonication on the dispersion of the nanofiller in the scaffolds was studied. This was expected to increase the mechanical properties of the final hybrid scaffolds. Firstly we analysed the effect of a double sonication on the dispersion of silicate and HAp particles on the polymer matrix. Figure 3-72 shows the elastic modulus of the different scaffolds after two fabrication processes: single sonication and double sonication. Unlike what happened with HAp, when silica was used as reinforcement the double sonication was able to properly disperse the filler and the elastic modulus increased, as seen in Figure 3-72. The highest elastic modulus observed is for the scaffolds filled with both HAp and silicate particles and double sonication. In this case the combination of both filler increased the Young's modulus from $4.7 \pm 1.0$ for pure PLLA to $6.9 \pm 1.0$ for the sample (90/95.5/0.5)-2son. 


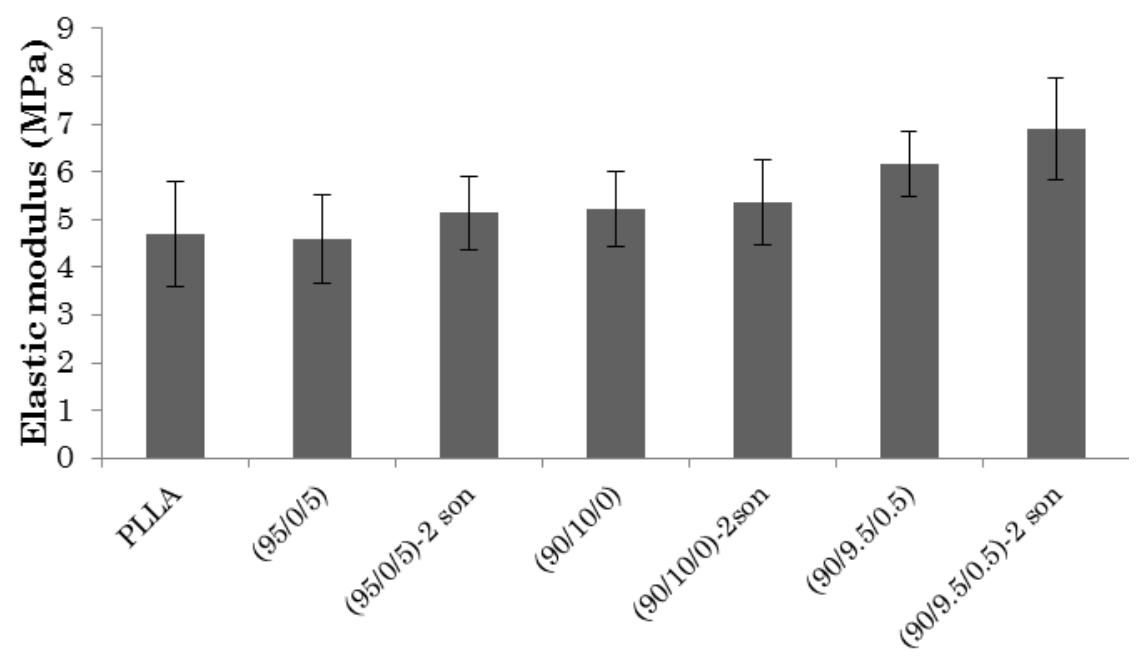

Figure 3-72: Values for the elastic modulus of compression of the PLLA-(95/0/5)-(90/10/0)-(90/9.5/0.5) scaffolds without sonication and after two sonication cycles.

\section{MORPHOLOGY}

The morphology of the hybrid scaffolds can be seen in the micrographs in Figure 3-73. As expected, they are similar to what described in section 3.1 .1$, p.64. They possess a macrostructure, with interconnected macro pores of about 150 $\mu \mathrm{m}$ diameter. Microporosity can also be observed at higher magnification, the micropores $(\sim 5 \mu \mathrm{m})$ are uniformly spread on over the surface of the scaffold. The macro and micro structure is similar for all kinds of hybrid composite, and are similar to the scaffold not filled or filled just with nanoparticles of HAp. The nanoparticles used to fill the polymeric matrix do not show up on the surface of the scaffolds. 


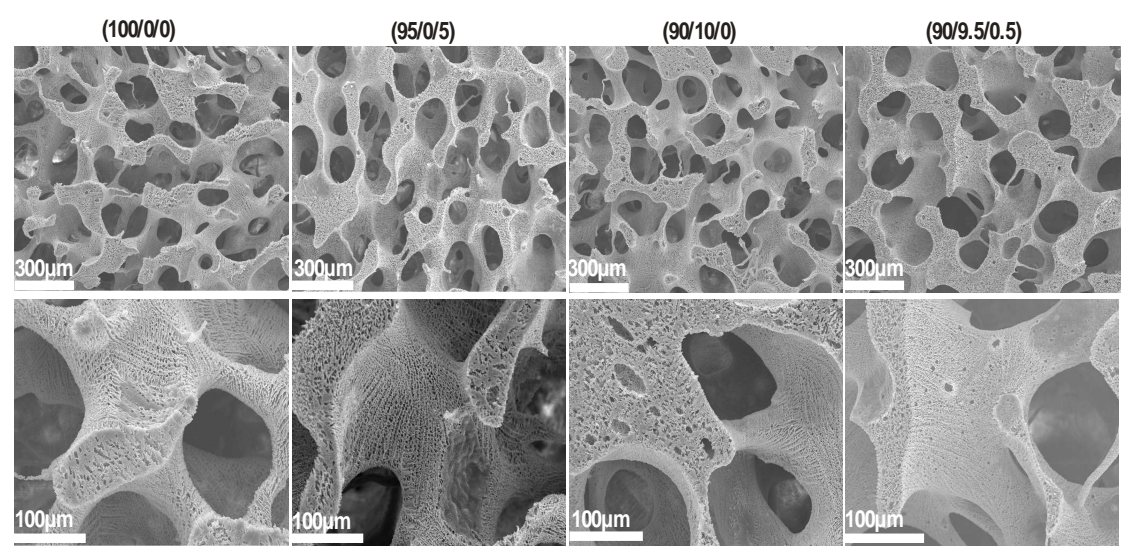

Figure 3-73: Scanning electron micrographs of the (100/0/0)(95/0/5)-(90/10/0)-(90/9.5/0.5) scaffolds.

\section{DIFERENTIAL SCANNING CALORIMETRY}

Figure 3-74 shows the DSC curves of the scaffolds. In this way, the effect of the characteristics of the filler on the calorimetric properties of the composites can be analysed. The enthalpic glass transition temperature (Tg), melting temperature $(\mathrm{Tm})$ and crystallinity after their fabrication process, are all shown in Table 3-14. The determination of crystallinity was done by an integration of the heat flow trace with respect to a linear baseline. The first DSC heat scan (Figure 3-74) does not show significant differences between the different samples. As shown on Table 3-14 Tg, Tm and crystallinity vary little between samples. 


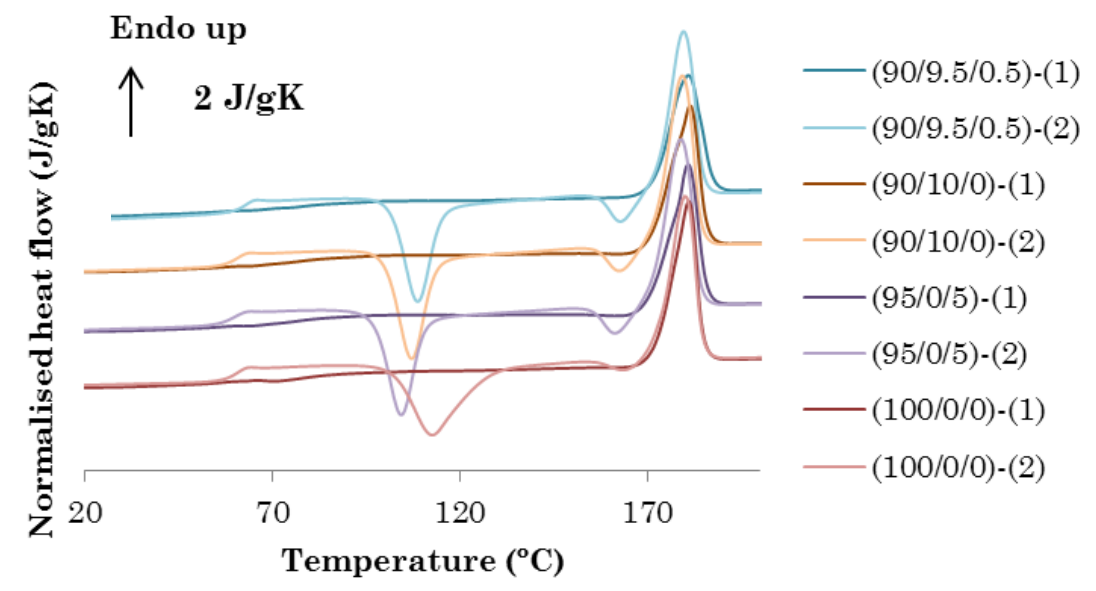

Figure 3-74: Normalised heat flow of the DSC first (1) and second (2) heating scans of the (100/0/0)-(95/0/5)-(90/10/0)$(90 / 9.5 / 0.5)$ scaffolds. The heat flow was normalised by the mass of the samples and the heating rate at $\left(10^{\circ} \mathrm{C} / \mathrm{min}\right)$. The arrow mark represents a unit of heat flow in $\mathrm{J} / \mathrm{gK}$.

Table 3-14: Tg, Tm and crystallinity of the (100/0/0)-(95/0/5)(90/10/0)-(90/9.5/0.5) scaffolds after the first scan.

\begin{tabular}{lccc}
\hline $\begin{array}{l}\text { (PLLA/HAp/Si) } \\
\text { content }\end{array}$ & Tg $\left({ }^{\mathbf{o}} \mathbf{C}\right)$ & $\operatorname{Tm}\left({ }^{\mathbf{o}} \mathbf{C}\right)$ & $\chi_{\mathbf{c}}(\mathbf{\%})$ \\
\hline$(100 / 0 / 0)$ & 74 & 181 & 60.4 \\
$(95 / 0 / 5)$ & 77 & 180 & 52.5 \\
$(90 / 10 / 0)$ & 81 & 180 & 47.8 \\
$(90 / 9.5 / 0.5)$ & 77 & 181 & 50.8 \\
\hline
\end{tabular}




\section{POROSITY}

No significant dependence of the scaffold porosity on the characteristics of the filler was found, as seen in Table 3-15. The global porosity, meaning micro and macro-porosity, calculated according to equation (5) seems be unchanged for the different scaffolds, regardless of the filler type. The porosity is still high, about $90 \%$. The measures were repeated 3 times for each case.

Table 3-15: Porosity of the diferent hybrid scaffolds.

\begin{tabular}{|c|c|}
\hline$\left(\mathrm{PLLA} / \mathrm{HAp} / \mathrm{SiO}_{2}\right)$ content & Porosity (\%) \\
\hline$(100 / 0 / 0)$ & $89 \pm 0.9$ \\
\hline$(95 / 0 / 5)$ & $88 \pm 0.5$ \\
\hline$(90 / 10 / 0)$ & $88 \pm 0.4$ \\
\hline$(90 / 9.5 / 0.5)$ & $88 \pm 0.4$ \\
\hline
\end{tabular}

\section{BIOACTIVITY}

In Figure 3-75 the SEM micrographs of the surface of the scaffolds after 21 days in SBF at high magnification are shown. A layer of HAp coating is observed on the scaffold. It has the typical cauliflower shape, like hemispherical-shaped apatite granules characteristic to biomimetic HAp from SBF ion precipitation. The granules contain numerous nano-sized apatite crystals $(149,171)$.

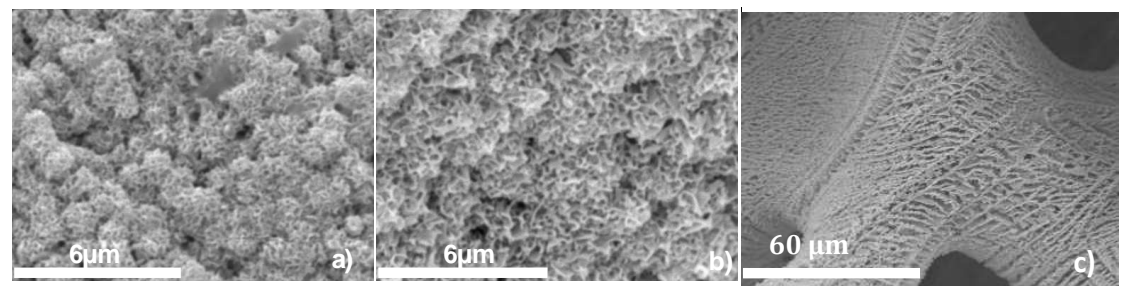

Figure 3-75: Scanning electron micrograph of a) (90/10/0), b) $(90 / 9.5 / 0.5)$ and c) $(95 / 0 / 5)$ scaffolds after 21 days in SBF 
In Figure 3-76 the micrographs of the internal and external surface of the (90/10/0) and (90/9.5/0.5) scaffolds after 14 and 21 days in SBF are shown. One can clearly see the influence of the filler on the ability of the samples to nucleate hydroxyapatite on its surface. As observed previously in the PLLA-CD scaffolds the nucleation of HAp coating on the surface of the scaffold does not cover the macrostructure. The layer nucleated on the surface of the scaffolds can be observed on the outside of the scaffolds after 14 days in SBF for both types of filler. The (90/10/0) samples do not show any significant HAp layer on its inside but only some nuclei (small white spots) appear on the microstructure after 14 and 21days in SBF. This contrasts with the inside of the (90/9.5/0.5) scaffolds where the layer of HAp gets bigger with time immersion (Figure 3-76). The microstructure of the (90/9.5/0.5) scaffolds cannot be seen, the HAp layer coating on its inside is dense and perfectly uniform. The (90/10/0) scaffolds present more HAp nucleated on the external surface of the scaffolds than in its inside, in particular after 14 days in immersion. Both types of scaffolds show, on the internal and external surface, a dense uniform layer of HAp hiding the microstructure. An exception has to be made for the external surface of the (90/9.5/0.5) scaffold which presents a uniform layer of HAp but the microstructure shape is still visible. 


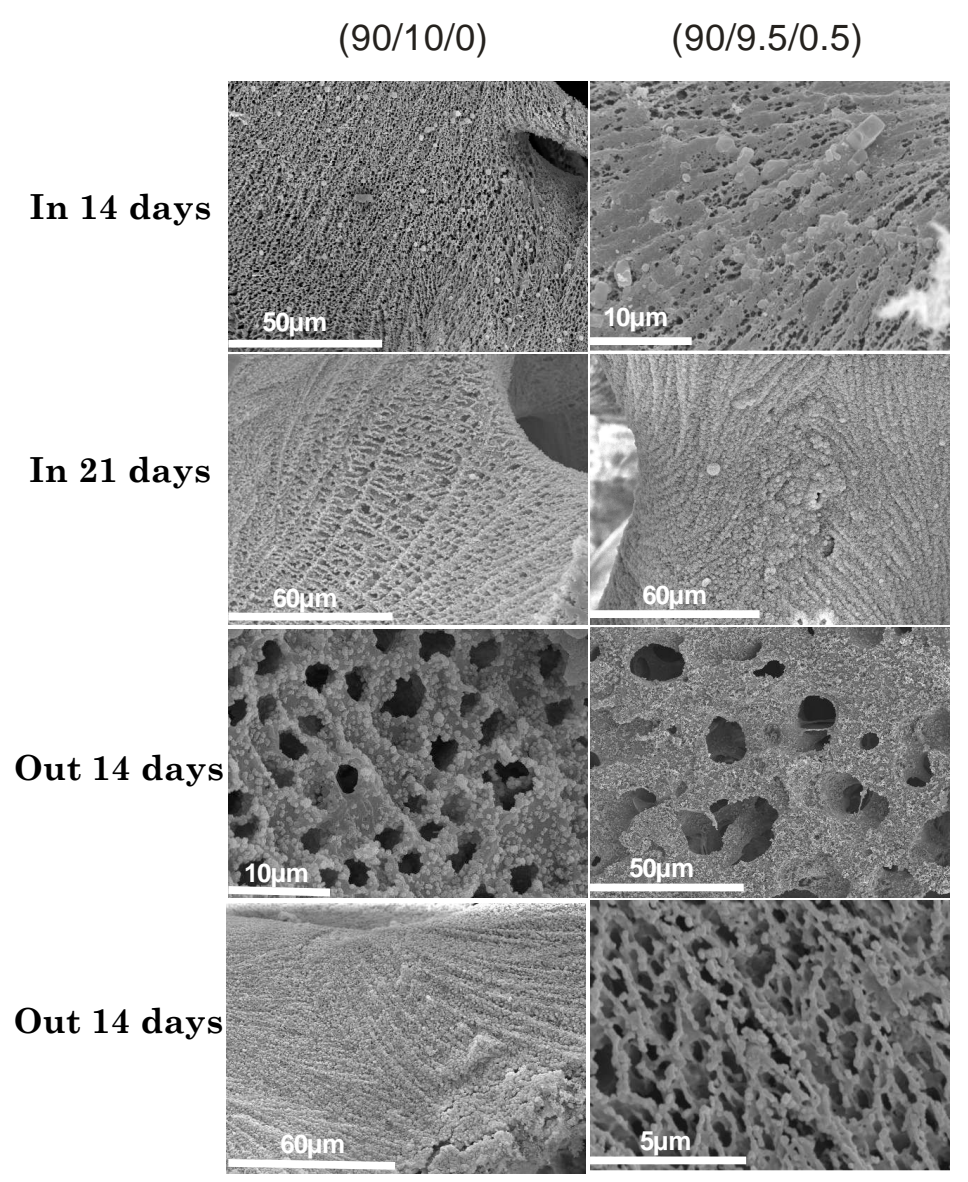

Figure 3-76: Scanning electron micrographs of the different hybrid scaffolds after 14 and 21 days in SBF.

Figure 3-77 shows the $\mathrm{Ca} / \mathrm{P}$ ratio of the different scaffolds after 14 and 21 days in $\mathrm{SBF}$. The $\mathrm{Ca} / \mathrm{P}$ ratio oscillates between 1.10 and 1.14 for the scaffolds immersed in SBF 14 days and between 2.06 and 2.36 for those in SBF during 21 days. Globally the $\mathrm{Ca} / \mathrm{P}$ ratio is higher after 21 days immersion than after 14 days. The scaffolds filled with HAp nanoparticles have a HAp layer coating on its inside and on its surface, with calcium and phosphate content similar to the physiological one (see the reference line Figure 3-77) after 14 days and 21 days in $\mathrm{SBF}$. 


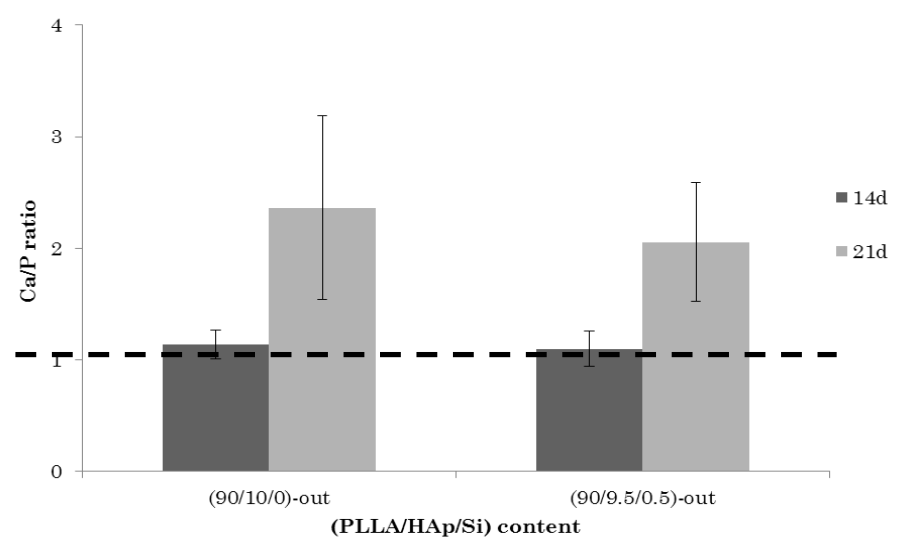

Figure 3-77: $\mathrm{Ca} / \mathrm{P}$ ratio of the layer of HAp coating on the surface of the (90/10/0) and (90/9.5/0.5) scaffolds after 14 and 21 days in SBF.

\section{CONTENT OF HYDROXYAPATITE COATED}

The content of HAp nucleated on the surface of the scaffolds as a function of the type of filler used to fabricate the composites was also evaluated by TGA analysis; Table 3-16 shows the percentage content of biomimetic HAp coating. Figure 3-78 shows the curves of the TGA experiment after 14 days in SBF. From these curves the percentage of residue is calculated, subtracting the percentage of nanofiller incorporated from the final content of inorganic component after thermal degradation. The percentage of HAp coating during the bioactivity test is the higher for the (90/10/0) scaffolds than then (90/9.5/0.5). When nanoparticles of HAp are incorporated the content of biomimetic HAp coating is the higher. 


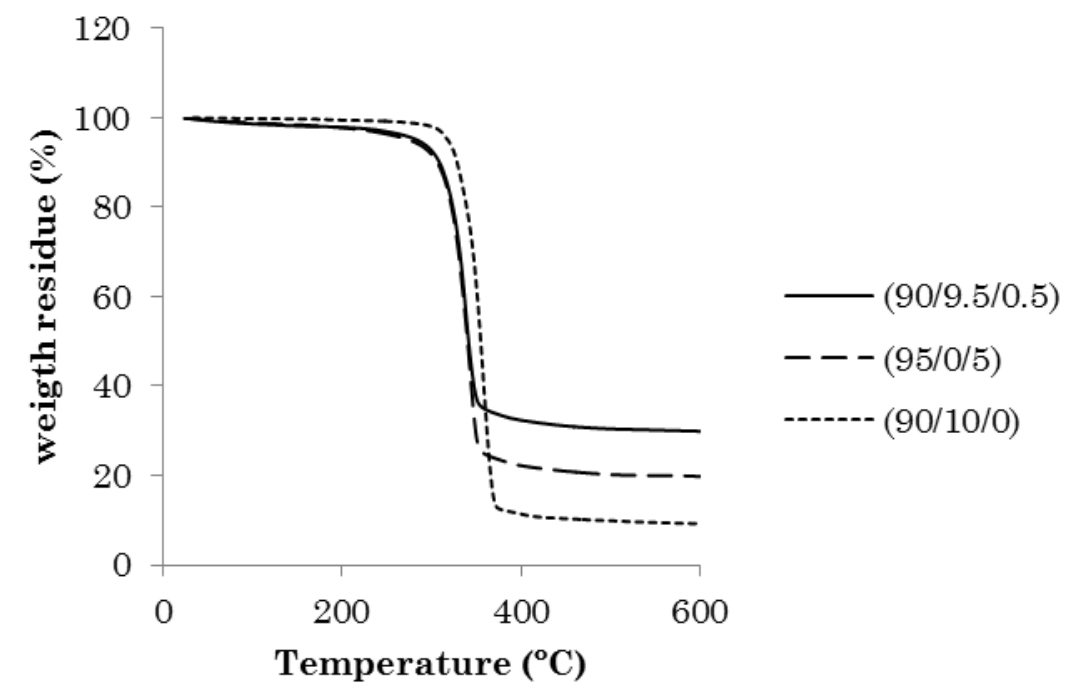

Figure 3-78: TGA curves of the different hybrid scaffolds after 14 days in SBF.

Table 3-16: Percentage content of biomimetic HAp coating on the surface of the scaffolds after 14 days in SBF.

\begin{tabular}{lc}
\hline (PLLA/HAp/SiO $)$ content & $\begin{array}{c}\text { Content of biomimetic HAp } \\
\text { coated (\%) }\end{array}$ \\
\hline$(95 / 0 / 5)$ & 11 \\
$(90 / 10 / 0)$ & 25 \\
$(90 / 9.5 / 0.5)$ & 16 \\
\hline
\end{tabular}





\subsection{OSTEOCHONDRAL BILAYER CONSTRUCT}

The main goal of this project is to design a bilayer scaffold each part functionalised to regenerate cartilage and bone tissue. The previous studies have permitted to get the optimum formulation of each part. Each part, consequently, has been characterized previously, only the morphology and mechanical properties of the biconstruct are presented.

The biconstruct is the combination of two scaffolds fabricated by freeze extraction and particle leaching. They are joined by a thin layer of polymer solution of $15 \%$ wt. of PLLA avoiding blood flow from the bone to the cartilage area. The up side of the biconstruct functionalised for cartilage repair is a 1:115\%PLLA-MED scaffold, $1 \mathrm{~mm}$ high. The bottom side is similar to the upside, functionalised for bone regeneration it has been incorporated $5 \%$ of bioactive nanoparticles of hydroxyapatite and is $6 \mathrm{~mm}$ high. So the biconstruct is a sandwich of $(100 / 0)$ and (95/5)- 1:1-15\%PLLA-MED scaffold.

The SEM micrographs, Figure 3-79, permit to appreciate how the two scaffolds are joined. Upside is the (100/0) scaffolds (1 $\mathrm{mm}$ high) and down the (95/5) scaffolds, bioactivated with nanoparticles of HAp. The biconstruct keep the macro and microstructure observed previously in the separated scaffolds The layer of polymer used to separate the two constructs do not present any pores, and is perfectly joined with both side. 


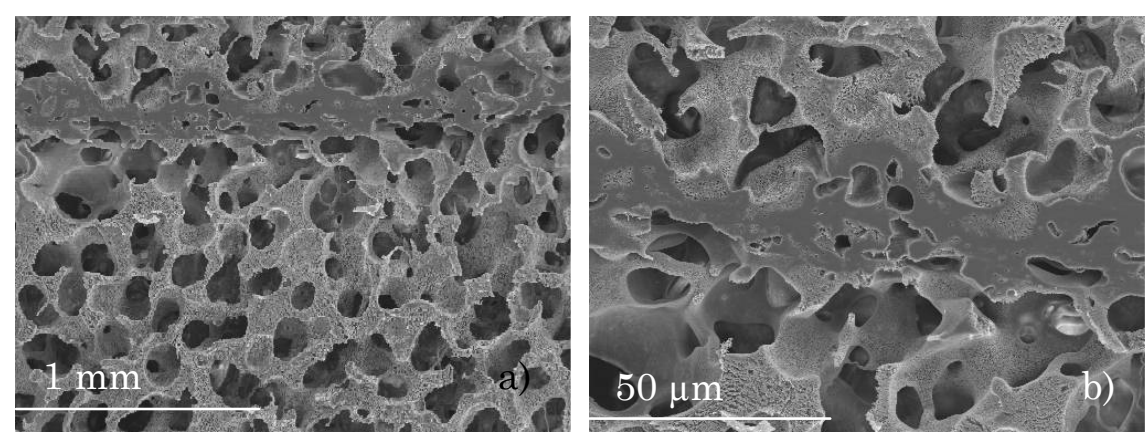

Figure 3-79: Scanning electron micrograph of the osetochondral contruct. 


\section{Chapter 4. Discussion}

\subsection{SCAFFOLDS FABRICATION: PROCESSING- MORPHOLOGY-PROPERTIES RELATIONSHIPS}

It can be seen how the PLLA concentration and porogen quantity influence the properties of the scaffolds.

\subsubsection{Effect on the scaffold properties}

Both micro and macro morphology are affected when the proportion of PLLA and dioxane is modified. The micropore is physically formed when dioxane crystals are leached by the ethanol. Indeed the dioxane crystals are formed due to the contact with the liquid nitrogen bath at $-180^{\circ} \mathrm{C}$ in the polymerpoor phase. The dispersion of the micropores depends on the affinity of the polymer and the solvent. A good solvent for PLLA, such as dioxane, allows good dissolution of the PLLA, and uniformly disperses the solvent molecules among the polymer chains. When phase separation occurs at low temperatures (it is produced during the freezing process with liquid nitrogen) dioxane crystals are formed separate from a polymer-rich phase, well distributed in this phase. The polymer-rich phase forms a low percentage of the solvent crystals due to the semicrystalline property of the polymer. The formation of the micropores due to the leaching of dioxane occurs uniformly distributed on the scaffold walls $(148,168)$. The more dioxane that is added to the reagent mixture the more the microporosity should be, as the polymer and solvent contents affect the process of separation of both by changing the viscosity of the mixture. Thus scaffold (5/95) has bigger micropores than scaffold (15/85). Microporosity is proportional to the volume of solvent crystals formed during the freezing 
process. The more dioxane is used the greater the quantity of micropores that are formed and the bigger they are. Dioxane increases microporosity through an increase in micropore dimensions. This effect is due to the fact that more molecules of solvent diffuse among the polymer chains as the solvent content increases (169-170). The limit of the micropores formation seems to be achieved at $20 \%$ of polymer content for the $1: 1$ scaffolds and at $18 \%$ of polymer content for the $1: 1.25$ scaffolds. It should be also noted that dioxane is also a good solvent to the porogen. As it comes into contact with the PEMA macrospheres they experience swelling. This absorption process leads to a reduction in the content of dioxane involved in the polymer/ solvent separation. If the microspheres of porogen swell until losing their spherical geometry and deform for being in contact with the neighbours then the trabeculae disappear and the macrostructure loses its regularity. That is the phenomenon observed principally for the $(5 / 95)$ scaffolds with higher dioxane content. The dioxane content absorbed by the PEMA spheres depends on the time duration of the mixing of the polymer solution and the porogen. The more dioxane present, the easier it is for the microsphere of porogen to come into contact with the solvent. Consequently, the microspheres will swell more rapidly, which would suggest the mixing process should be completed more quickly in order to avoid the deformation of the structure of the final scaffolds. As this time parameter is not exactly the same for all the samples and polymer concentration solutions it is not possible to know how much dioxane has been absorbed by the porogen. Consequently it is easy to understand why the maximum micropore formation is achieved at the lower concentration of polymer for the 1:1.25 scaffolds compared to the 1:1 scaffolds, as they present a higher content of porogen susceptible to absorb more dioxane. These observations can be made for both brands of PLLA. PLLA-CD and PLLA-MED present the same average micropore dimension, see Figure 
3-6. Through the freeze extraction process we have been able to produce macropore walls with well controlled dimensions, as dioxane crystals were eliminated once PLLA-rich phase was frozen. The normal process followed in literature is the solvent casting. The main disadvantage of this technique is that the resulting scaffold does not have a uniform microporosity on its macropore walls because the polymer contracts as the solvent evaporates, leaving defects in the micropores.

Macroporous structure depends on another parameter, the porogen quantity. The porogen macrospheres (average diameter of 100 to $150 \mu \mathrm{m}$ ) were incorporated to the polymer solution. After dioxane has been removed the temperature of the ethanol bath is increased to make the PEMA removal easier. As ethanol is able to dissolve the porogen, the spaces occupied by the porogen macrospheres are replaced by macropores. The higher the polymer/ porogen ratio the more viscous is the mixture. Consequently the homogenization with PEMA will be difficult, and the dioxane solution and porogen will spend more time in contact with one another. As was previously noted, the greater the time during which the porogen and the dioxane are in contact, the more the microspheres will swell and increase in size. That explains why the macropores are larger than the initial porogen dimension. It also explains why the scaffolds with the highest content of porogen, series 1:1.25 presents a macroporous structure with very thin pore trabaculae or hardly existing. The macrospheres of porogen had so much time to swell that they are in contact one with another. That also explains why the (5/95) scaffolds present the biggest macropore dimension; it has the highest dioxane content in the polymer solution, so the quantity of solvent available to cause the microspheres of porogen to swell is also highest. The same observations can be made for both brands of PLLA. Furthermore PLLA-CD and PLLA-MED present the same average macropore dimension. 
The calorimetric properties do not seem to be affected by any change of parameters such as the polymer concentration of the polymer solution or the polymer / porogen ratio. In fact Tg, Tm and crystallinity are almost the same in the different scaffolds. The differences among the samples observed for the $\mathrm{Tg}$ measurements are due to the error in determining the glass transition temperature. However, each type of PLLA has a different $\mathrm{Tg}$, Tm and particularly crystallinity after the freeze extraction process. Medical use PLLA scaffold is more crystalline than the industrial use PLLA scaffold. In order to facilitate the processing in industry some additives, such as a small quantity of PDLA units, are normally added to the PLLA. Those industrial additives are to impede PLLA crystallisation which would explain the higher crystallinity found in the purest PLLA-MED $(134,171,172)$. However, the exact nature of the used additives is unknown as the manufacturers try to protect their fabrication protocols.

In the second scan for both PLLAs a crystallisation peak, or even two, appears. It can be concluded, that, when the cooling process is fast the material cannot crystallise up to maximum capacity, and it continues with its crystallisation after the glass transition temperature. The same should happen in the freeze extraction process and we would expect to have scaffolds with very low crystallinity. However, the absence of any crystallisation peak in the first scan means that the process of fabrication of the scaffolds permits them to crystallise up to the maximum capacity. In fact, when we put them in the ethanol baths at $40^{\circ} \mathrm{C}$, to remove porogen spheres, we are close to the Tg of PLLA, ethanol plasticises the PLLA and facilitates the incorporation of polymer chains to ordered crystalline structures. Thus, changes of scaffolds mechanical properties are due to the different porosities and morphologies and not to changes in crystallinity. 
These differences of macro and micro structure according to the changes in polymer/ solvent or polymer/ porogen content have, as expected, an influence on the mechanical properties. As observed on the SEM pictures (see Figure 3-1Figure 3-2,Figure 3-3,Figure 3-4) the scaffolds with the smaller pore throats between macropores present the highest elastic compression modulus, due to the support of the pore walls. The bigger the cell walls, the more they are able to sustain the compression solicitation. The microstructure has also an influence on the mechanical properties, as micropores can be considered as closed-cells (166). The greater the proportion of cells on the surface walls of the scaffolds, the less they are able to sustain the compressive forces. The dimension of the cells can also influence the mechanical properties. It has been observed that the evolution of the morphology of the scaffold is related to the composition, which has a direct effect on the mechanical properties. The (5/95) scaffold has a macrostructure with thin pore walls or no wall. The yield point for these scaffolds occurs at the smallest strain (Figure 3-8 and Figure 3-9). When the polymer content increases more material is used to form the structure, and consequently the pore walls are thicker. Then, the scaffolds are more resistent to compressive forces. As a result the yield point of the scaffold $(15 / 85)$ is shifted toward higher strain and the elastic modulus is higher than the ones of the (10/80) scaffolds. One of the major differences between PLLA-CD and PLLA-MED is in the mechanical properties. Scaffolds made with industrial PLLA present higher elastic modulus but lower yield point limits than the medical PLLA (Figure 3-11). The PLLA in some point seems to be more resistant to deformation than industrial PLLA. Medical grade PLLA is more crystalline than industrial PLLA. More crystallinity means a greater quantity of reinforcing crystallites and consequently better mechanical properties. 


\subsubsection{Selection of a composition}

The best candidate for both cartilage and bone regeneration could be the (15:85) scaffold. One of the main requirements for scaffolds in bone and cartilage tissue engineering is to sustain the load applied, while the tissue repairs itself. In this study the scaffolds presenting the highest elastic moduli are the scaffolds with 1:1 polymer / porogen ratio and particularly that with a $15 \%$ of PLLA content. The elastic modulus obtained for this scaffold ( $\sim 9 \mathrm{MPa})$ is of the same order of magnitude of that of cartilage (5). However, it is smaller than that of bone (89). It is a useful study to determine how to increase the mechanical properties of PLLA. Especially for the scaffolds made with PLLA-MED, the one expected to be used in the biological experiments due to its medical grade, but presenting a smaller elastic modulus ( $\sim \mathrm{MPa})$.

\subsubsection{Partial conclusions}

Freeze-extraction combined with particle leaching is one method to obtain highly porous scaffolds, with double porosity. The freeze-extraction leads to the formation of microporosity and the particle leaching leads to a well interconnected macroporosity. PLLA is easily shaped by this technique to obtain scaffolds with high mechanical properties with an elastic modulus close to that of articular cartilage. It is possible to adjust the micro and macropore size (5 and $150 \mu \mathrm{m})$ by changing parameters such as polymer concentration in the polymer solution and the polymer/ porogen ratio. Decreasing the polymer content in the polymer solution results in the proportion of solvent being higher, and so the microporosity dimensions increase. Increasing the porogen content it was possible to generate more macropores. In this study two types of PLLA have been used, one for industrial process and another for medical use. The crystallinity, $\mathrm{Tg}$, Tm of the 
medical use PLLA are higher than those of the industrial PLLA. Otherwise the calorimetric properties are not affected by the changes of polymer concentration or polymer/ porogen ratio. The scaffold 1:1-(15/85)-PLLA-MED is a good candidate for a possible use in osteochondral regeneration as its morphology is adequate for cells and nutrients flow and it has the ability to sustain the required load. 


\subsection{DEGRADATION EXPERIMENTS}

In the static case the degradation of the polymer chains can be detected by GPC analysis from the first week (Figure 3-24 p.98). The degradation seems to be superficial and should start from the pores on the surface of the scaffolds. It is not clearly observed that the degradation starts from the surface of the micropore (Figure 3-14, p. 85). Nevertheless, microCT images show that the macropore dimension changes which is consistent with the observed because permeability increase with degradation time from the first week (Figure 3-18, p.92). Usually the effects of degradation of PLLA can be observed between 10 month and 4 years (174). Of course this result depends on parameters such as the initial crystallinity or the porosity of the sample. But it has been found that porous scaffolds degrade more rapidly than bulk samples (175).

The mechanical properties are also affected. As shown in Table 3-6, p.90, the Young's modulus slightly decreases with the time of degradation. However, changes in the pores collapse zone were the most notable effects of the degradation process observed in this study, as the yield point occurs at lower strain with degradation (Figure 3-16 p. 89). This is certainly due to the degradation occurring in surface, leaving the interior of trabeculae not degraded, which, consequently gives resistance to the structure. As demonstrated in the GPC curves at low degradation times the molecular weight had not yet decreased significantly (Figure 3-25, p.99). Kang et al. observed a linear decrease in the compressive strength from 10.5 $\mathrm{MPa}$ to $7 \mathrm{MPa}$ in 6 weeks of degradation in SBF of porous PLLA scaffolds (176). Tsuji et al. studied the hydrolytic degradation of PLLA films in PBS as a function of their crystallinity. It was observed that the Young modulus of the PLLA specimens decreased monotonously in the first 8 month $(128,129)$. 
The evolution of the mechanical curves changes clearly in the dynamic case. In the dynamic study the curves start from the plastic zone as the dynamic load applied during the test start to deform plastically the samples (Figure 3-32, p.107). As the time of degradation increases the densification zone occurs at lower stress.

The weight loss is the less notable effect in the degradation test (Figure $3-15$, p.87), as it could not be seen in this case, also due to the deposition of salts from the medium (Figure 3-31, p.106).

The scission of the polymer chains can be observed as the samples get less and less thermally stable when degradation time increases (Figure 3-27 and Figure 3-28, p102). This result is also related to the decrease of molecular weight and the narrow molecular weight distribution.

The crystallinity of the scaffolds after the first heating being higher than the second one permits us to say that the freeze extraction process generates highly crystallized scaffolds (50\%) (Table 3-7 and Table 3-8, p.111). It is known that degradation of PLLA starts with the amorphous region (129). During degradation due to the scission of the ester group leading to the formation of carboxyl and hydroxyl endgroups their density increases in the amorphous region $8(114,124)$. These functional groups are able to catalyse the degradation process of the amorphous region. Density of hydrophilic terminal groups increasing can stimulate the diffusion of water in the material, making faster the degradation of the amorphous regions. The presence of a higher fraction of carboxylic and hydroxilic groups could act as plasticizers of the main chains lowering Tg of the degraded samples. It is not our case, as the decrease of $\mathrm{Tg}$ cannot really be observed. Neither the Tg decrease in Tsuji et al. studies is remarkable after one year of hydrolytic degradation in PBS (128). The effect of the degradation during the first DSC isotherm cannot be seen but in the second one. It could be because the chains of the 
amorphous region degraded remain without any mobility, entrapped between the crystalline regions, as our materials are quite crystalline. Then, we do not observe any increase in the crystallinity as the time of degradation increases However, the second heat permits to observe the degradation as crystallinity increases when the time of immersion increases. In the second heat scan we calculate the ability of the material to crystallise from the melt. As the density of short chains increases, their organization into crystallites is easier. This phenomenon is better observed in the static case as this experiment was performed over a larger time period.

\subsubsection{Partial conclusions}

Static and dynamic degradation of PLLA affects the mechanical properties as the yield point decreases with degradation time furthermore the robustness of the scaffolds decreased in the permeability test. However, in the static case, the elastic modulus decreases very slightly with degradation time. The effect of dynamic degradation is only seen in the densification zone as during the experiment the scaffolds were collapsed.

The micro and macro porosity seemed to be affected by the degradation. However, changes in morphology are quite slight as corresponds to surface erosion.

The degradation attacks the amorphous phase that keeps entrapped in the samples confined in the crystalline region, and no changes in crystallinity or mass are observed during the degradation experiment. Freeze extraction fabrication process leads to scaffolds highly crystalline that are able to maintain their morphology after 24 weeks of static degradation and 6 weeks of dynamic degradation. 


\subsection{REINFORCEMENT AND BIOACTIVITY OF THE MATERIAL}

\subsubsection{Effect of the filler content on the membrane's properties}

The freeze extraction process produces membranes with $10 \mu \mathrm{m}$ micropores as shown in Figure 3-44, p.121. The micropores are homogenously dispersed on the surface of the membranes. All membranes exhibited the same morphology, regardless of the filler content. The pores are produced by the phase separation when both dioxane and PLLA crystallise from the solution.

When producing a composite, the HAp nanoparticles are suspended in the solution. Crystallisation of PLLA traps some of these particles but other particles are expected to remain between different dioxane crystals or at the interfaces between dioxane and PLLA in the solid sample when immersed in liquid nitrogen. DSC experiments included a heating scan that gives information about the crystalline structure obtained after freeze extraction process. This scan was performed between 0 and $210^{\circ} \mathrm{C}$. At the end of this scan the sample is melted. The second heating scan was carried out to characterize the structure of the polymer crystallized from the melt. The differences between the second and the first scan can be explained by the formation of crystalline nuclei during physical ageing at temperatures below $\mathrm{Tg}$ that has been proven in PLLA and in other semicrystalline polymers as well (128). An increased number of nuclei at the beginning of cold crystallization speeds up the process; as a result the observed crystallisation peak is sharper and shifted towards lower temperatures. The changes in shape and position, in Figure $3-46$, p.125, for cold crystallisation peaks with increasing HAp content demonstrate the nucleating effect of the HAp nanoparticles. 
The deviations of the calcination test (Table 3-9, p.123) can be an indication of an imperfect dispersion of particles, or the leaching of a part of the particles that remained in the solvent during phase separation. Dynamic-mechanical experiments (Figure 3-47, p.128) are also highly dependent on the thermal treatment to which the sample is subjected. The increase of the storage modulus with HAp content shows the reinforcing role of the HAp particles. The reinforcing effect of the nanoparticles is clearly shown in the semicrystalline samples both below and above the main relaxation of the amorphous phase.

The morphology of the membranes seems not to be affected by plasma treatment. After oxygen plasma treatment, as shown in Figure 3-45, p.122, some kind of abrasion defects can be seen on the surface of the membranes, and do not appear on the membranes without plasma treatment. Surface abrasion that was already found in plasma treated PLLA by Wang et al. can allow HA particles to appear at the pore walls.

The biomimetic layer of HAp nucleated on the surface of the scaffolds present the typical cauliflower with needle like granules (Figure 3-54, p.140). Although plasma treatment increases the bioactive potential of pure PLLA, as shown in figure 5.6, its effect is not seen before 21 days in SBF. A faster process is needed to avoid degradation of the polymer before its osteo-integration. After 21 days in SBF or in the implantation site PLLA starts the degradation process (as seen in the case of the scaffolds) and consequently its mechanical properties start to decrease too early making the material useless for bone tissue engineering. Consequently it is interesting to generate a layer of HAp at shorter time of $\mathrm{SBF}$ immersion (7 days in SBF), an effect that has been obtained in the membranes by the addition of the HAp particles. Figure 3-49, p.132, shows the effect of the HAp nano-filler content after 7 days immersion. After this time the 
PLLA samples show no signs of apatite layer even when treated with plasma, but the rate of deposition increases with HAp content and samples containing more than $10 \%$ wt. HAp nano-particles show a smooth coating on the composite surfaces. Plasma treatment further enhances the rate of deposition, and the thickness of the layer increases in such a way that biomimetic apatite fills the whole volume of the micropores. Results obtained after different immersion times in $\mathrm{SBF}$ (7, 14 and, 21 days) are similar. Plasma treatment increases the wettability of the material (179), which increases the interaction between the ions present in SBF and the surface of the material. Consequently plasma treatment accelerates the process of precipitation of the ions on the surface of the material. This effect is more pronounced when HAp is mixed with PLLA. Surface abrasion produced by plasma permits the particles to show up on the surface of the composite. The $\mathrm{Ca}^{2+}$ and $\mathrm{PO}_{4}{ }^{2-}$ ions release within the $\mathrm{SBF}$ solution speeds the process of HAp nucleation by creating positive or negative charges on the surface due to the formation of ions in the aqueous medium.

The $\mathrm{Ca} / \mathrm{P}$ atomic ratio of the biomimetic apatite calculated for all the samples is higher than the physiological HAp one, ranging from 1.63 to 2.54 (Table $3-12$, p.133). The membranes with HAp incorporated at day 0 , in which the $\mathrm{Ca} / \mathrm{P}$ atomic ratio measured corresponds to the HA nanoparticles added as filler, have the highest ratio, and the ratio observed does not match that of stoichiometric hydroxyapatite claimed by the supplier. For the same immersion time membranes previously exposed to plasma present a ratio closer to the physiological one in comparison with membranes that had not been exposed to plasma. In general, the ratio that is closest to the physiological one is for the plasma treated membranes prepared with nano-filler after 7 days in SBF. 


\subsubsection{Effect of the filler content on the scaffold's properties}

As can be seen on SEM micrographs in Figure 3-73, p.161, the morphology of the scaffolds is not affected by the variation of the filler content. The macro and micropores still present the same dimension, around $120 \mu \mathrm{m}$ and $5 \mu \mathrm{m}$, respectively. The porosity is high, around $80 \%$, regardless of the filler content. The macropores are well interconnected which could provide cells and nutrients flow to promote tissue regeneration.

Globally the Young's modulus is decreasing or maintained constant when filling the scaffolds with nanoparticles of HAp, in spite of the increase of the storage modulus with the increase of HAp nanoparticles in the membranes. This could be explained by possible aggregation of the particles of nanofiller in the polymeric matrix. However, an improvement of the mechanical properties of the scaffolds was expected with the incorporation of the nanoparticles as previously described in literature (161). The particles act as reinforcement in all direction, provided that the previous sonication of the particles in the polymer solution disperses them uniformly in the scaffolds. The chemical, physical and mechanical interface properties between the matrix and the filler have very strong influence on the properties of the final composite material. This depends on the wettability of the particles and the interaction between the filler and the polymeric matrix. As the superficial tension of PLLA and the particles are both negative it cannot be expected a good interaction between them (184). The aggregation of the nanoparticles of HAp can lower the surface interaction between the filler and the matrix. This can explain the decrease of the elastic modulus of the scaffolds filled with particles of HAp.

After 14 and 21 days in SBF the Young's modulus decreases even more than that corresponding to the scaffolds filled with 
nanoparticles not immersed in SBF. This is due to the degradation of the PLLA by hydrolysis in SBF. It can be seen that the PLLA degrades by hydrolysis through the presence of esters where scissions take place. In the crystallized PLLA materials the chains in the amorphous regions are predominantly hydrolysed $(180,181)$. However, an increase of the elastic modulus is observed for the scaffolds with filler immersed 14 and 21 days in SBF. The modulus is higher after 21 days than 14 days. This may be due to the effect of the HAp coating which acts as reinforcement. So the filler increases the bioactivity of the material. The layer of HAp coating on the surface of the material acts as a framework. This framework protects the matrix from more degradation as it isolates the material from the medium.

It seems that the coating obtained is hydroxyapatite. The $\mathrm{Ca} / \mathrm{P}$ ratio is close to the physiological one after 21 days in SBF. The variations obtained for the $\mathrm{Ca} / \mathrm{P}$ ratio is due to the crystals activity in the aqueous medium. The precipitation of ions present in SBF can lead to the formation of combinations other than $\mathrm{Ca} / \mathrm{P}$. Ions can proceed from an intermediate precursor phase with a transitory existence like octacalcium phosphate or tricalcium phosphate, but these transitory precursors present a $\mathrm{Ca} / \mathrm{P}$ ratio smaller than 1.67 (Table 3-13, p.142). This deviation is due to the presence in the crystal lattice of vacancies and ion substitutes such as chloride (182). Small zones were carefully selected to avoid measuring the gaps between cauliflowers where the electron ray is not coherently dispersed. However, the possibility that the dispersion values are a result of imprecision in the measurement accuracy cannot be discounted. The formation of HAp becomes the most kinetically favorable phase in SBF with time (117). The signals detected by EDS analysis present small peaks of $\mathrm{Na} / \mathrm{Cl}$ but their concentrations are not 
significant. The FTIR corroborates that the layer coated on the scaffolds is HAp (Figure 3-66 and Figure 3-67, p.154).

It is clear that with increasing immersion time, the layer coating increases. Previous works in this field yielded the same conclusion (102). The layer is clearly denser on the surface than inside the scaffold due to difficulty in the diffusion of ions inside the pores. Furthermore in some areas the microporosity structure can still be observed. This is a consequence of the precipitation of the ions which is generated by the contact with the matrix. Indeed the hydrolysed COOgroups of the PLLA or the HAp nanoparticles incorporated present a negative charge leading to the attraction of the positive ions and so initiate the formation of the layer of HAp. This combination is made via ionic and/or polar interactions (183). The surface of the scaffolds presents a denser layer of HAp, because of a better surface contact. Plasma treatment increases the bioactive potential of pure PLLA (120). The plasma attacks the surface of the material and so creates an abrasive surface, increasing the roughness and so permit the precipitation of ions. This causes nanoparticles of HAp to show up (Figure 3-45, p.122) on the surface, promoting the formation of charges on the surface of the sample (184). Plasma treatment and incorporation of nanoparticles of HAp are effective processes in improving the bioactivity of PLLA.

After 21 days in SBF or in the implantation site PLLA starts the degradation process and consequently its mechanical properties start to decrease too early making the material useless for bone tissue engineering. Consequently it is interesting to generate a layer of HAp with a shorter immersion time in SBF (7 days). 


\subsubsection{Effect of filler type}

The nature of the different type of filler used as hydroxyapatite nanoparticles, silicates or the mixture of both does not affect the morphology of the scaffolds.

The crystallinity presented here is the consequence of the reorganization of the polymer-rich phase during the phase separation and also the reorganization of the polymers chains in ethanol at $40^{\circ} \mathrm{C}$ during particle leaching. Even if the particle leaching was done below glass transition temperature the ethanol helps to the chains to reorganize acting like a plasticiser. The crystallinity decreases from $60 \%$ to $50 \%$ with incorporation of the nanoparticles. That means that a part of the crystallization of the polymer-rich region is not occurring because of the presence of the nanoparticles due to physical hindrance. The crystallinity does not seem to be influenced by the type of nanofiller.

It can be observed, in Figure 3-72, p.160, how the double sonication results in the enhancement of the elastic modulus of the scaffolds filled with 10\% of HAp and both HAp and silica. Thus, the elastic modulus increased significantly, in the order of a $30 \%$ when the silica nanoparticles are combined with the HAp particles. It is hypothesised that the silica nanoparticles interact with the nano particles of HAp. The agglomerates of HAp bond with smaller particles of silicates. Consequently the surface of interaction between the filler and the polymer matrix increases. The surface interaction is even higher if the silicates are hollow nanoparticles.

The modulus obtained for these scaffolds, considering the very high porosity (90\%) and its polymeric nature, is high (around $10 \mathrm{MPa}$ ). This modulus compared to other porous scaffolds is similar or higher. Wagoner et al. reviewed polymer calcium phosphate scaffolds compressive strength as a function of 
porosity and did not report any research presenting an elastic modulus higher than $4 \mathrm{MPa}$ (71). Wang et al. present PLLA/ silane modified hydroxyapatite porous scaffolds with a compressive modulus not greater than $1 \mathrm{MPa}$ (162). The scaffolds do not really need to present the mechanical properties of bones as it is intended as a support for the regenerating tissue. Attention should also be paid on the enhancing bone binding efficiency of the scaffold.

Pure PLLA is not bioactive as it does not present any HAp nucleated on its surface (Figure $3-49$, p.132). In order to increase the bone binding of the scaffolds bioactive nanoparticles such as HAp and silicate have been added $(162,185)$. The incorporation of $\mathrm{SiO}_{2}$ or HAp only increases the bioactivity of the scaffolds on the outside surface. The combination of both $\mathrm{SiO}_{2}$ and $\mathrm{HAp}$ contributes to nucleate HAp after 21 days in SBF on all the surface of the scaffold (Figure 3-75, p.163). The thermogravimetry allows quantification of the amount of HAp nucleated on the surface of the samples. The layer accumulates in greater proportions for the scaffolds filled with $10 \%$ of HAp nanoparticles than those with both HAp and silica. The scaffolds (90/9.5/0.5) accumulate less proportion of HAp but the layer is observed on the internal surface and the external surface of the scaffolds. The layer observed is similar to physiological hydroxyapatite as $\mathrm{Ca} / \mathrm{P}$ ratio is close to 1.67 . On the internal surface this ratio is bigger because the inside of the scaffold is difficult to rinse after SBF soaking, and so some residue interferes with the measure.

\subsubsection{Partial conclusions}

i. Freeze extraction results in PLLA/ HAp membrane composites with a good dispersion of HAp particles and bioactive properties. The storage modulus increases with the filler content at temperatures above and below 
the glass transition of the amorphous phase. Particles act as a nucleating agent for PLLA modifying the crystallisation kinetics whereas glass transition temperature of PLLA is independent of the filler content. The bioactivity is enhanced by the presence of the HAp particles more than by plasma treatment; indeed the nucleation of a biomimetic HAp layer on the surface of the nano-composites when immersed in SBF is more effective than on PLLA membranes. This study examines two independent ways to increase the bioactivity of PLLA membranes: nanoparticles of HAp content and plasma treatment. Our results show that membranes demonstrating the best properties for use in bone regeneration are that with a $15 \%$ of HAp nanoparticles and previously exposed to plasma. These membranes demonstrate good bioactivity, they are able to nucleate a layer of $\mathrm{HAp}$ with a $\mathrm{Ca} / \mathrm{P}$ ratio similar to the physiological one, after only 7 days in SBF, and so avoid a possible degradation of the material.

ii. Despite the decrease of the Young modulus of the scaffolds when incorporating only 5\% of HAp, and the degradation of PLLA in SBF, it is observed that when immersion time in SBF increasing, the thickness of the layer of HAp coating also increases. Combining the incorporation of nanoparticles and a previous plasma treatment it is possible to obtain a uniform layer of HAp coating on the surface after only 14 days in SBF. The formation of some nuclei on the internal surface is observed after 21 days in SBF.

iii. The sonication process reduces the particle agglomeration and increases the specific surface with the polymer matrix and therefore a better interaction 
between the particles and the matrix is obtained. The utilisation of $\mathrm{SiO}_{2}$ particles in low quantity together with an amount of HAp particles results in an increase of the elastic modulus in compression. This was due to an augmentation of the specific surface between PLLA and nanofiller, due to the silica nanoparticles incorporation. The composite material made with PLLA as matrix and reinforced with $10 \%\left(\mathrm{HAp} / \mathrm{SiO}_{2}\right)$ wt. with the ratio $\left(\mathrm{HAp} / \mathrm{SiO}_{2}\right)$ of $(95 / 5)$ showed a bioactivity on the surface after 21 days soaking in SBF. This study led to the fabrication of a highly porous scaffold, with a bioactive surface, an elastic modulus of nearly $10 \mathrm{MPa}$, and with a morphology that allows cell and nutrients flow. 


\subsection{BILAYER CONSTRUCT}

The join observed between the two functionalised sides is greatly assembled to both scaffolds. This is due to the dioxane used in the polymer solution to bond both side. The dioxane dissolved the PLLA in the scaffolds consequently permits to combine the PLLA of the scaffolds and the join when the dioxane is removed.

This bi-layer scaffold presents a similar elastic modulus according to the cartilage phase. Bioactivity of the bone layer is improved by filling the PLLA matrix with HAp nanoparticles. The bi-construct has a very high porosity over $80 \%$ with macro and micropores, $100 \%$ biodegradable. The nanoparticles of HAp do not increase the elastic modulus but bring to PLLA a better bone bounding ability. This design was one signed for, but it does not exclude other combination such as including $\mathrm{SiO}_{2}$ particles for the bone part.

At present, the viability of the final osteocondral construct is being studied in vivo; several replicas were implanted in femoral condyle of sheep by our collaborators at the Laboratorio de Ortopedia Experimental Área de Terapia cellular of the University of Navarra. 



\section{Chapter 5. Conclusions}

- Freeze-extraction combined with particle leaching was a method with which it was possible to fabricate porous scaffolds of PLLA with a microstructure around $5 \mu \mathrm{m}$ and macrostructure around $150 \mu \mathrm{m}$. The porosity obtained was high, over $80 \%$ with spherical pores interconnected.

- It was possible to evaluate the influence of the microporosity dimension on the properties of the scaffolds varying the polymer/solvent ratio between the limits of fabrication 5\% and 20\% wt. of PLLA. Macroporosity and pores throats were varied by changing the polymer/ porogen ratio. The limits of this ratio were established between $1: 1$ and 1:1.25.

- The calorimetric properties were not affected by the changes of polymer concentration or polymer/ porogen ratio, as in the porogen leaching PLLA crystallized up to its maximum. The observed changes in morphology had influence mainly in the elastic modulus of the scaffolds that varied from $0.1 \pm 0.1$ to $8.9 \pm 0.8 \mathrm{MPa}$ for industrial PLLA and from $0.01 \pm 0.03 \mathrm{MPa}$ to $6.1 \pm 0.9 \mathrm{MPa}$ for medical grade PLLA and in a lesser extent in the porosity.

- The scaffolds made with 15\% wt. of PLLA and 1:1 polymer ratio with an elastic modulus of $8.9 \pm 0.8 \mathrm{MPa}$, for industrial PLLA and 3.5 $\pm 0.6 \mathrm{MPa}$ for medical grade PLLA was selected for the final design of the osteochondral construct.

- Static and dynamic degradation of PLLA produces the scission of the ester bonds giving rise to a higher fraction 
of low molecular weight chains. The evolution of the molecular weight distribution function indicated that PLLA suffers surface erosion as for almost all the degradation times there is still a quite big fraction of long chains. Chain scission affects mostly the mechanical properties. In the static case, the elastic moduli decreased very slightly while the yield point decreased to a bigger extent. Furthermore the robustness of the scaffolds decreased in the permeability test. The effect of dynamic degradation is only seen in the densification zone as during the experiment the scaffolds were collapsed.

- The micro and macro porosity seemed to be affected by the degradation. However, changes in morphology are quite slight as corresponds to surface erosion.

- The degradation attacks the amorphous phase that keeps entrapped in the samples confined in the crystalline region, and no changes in crystallinity or mass were observed during the degradation experiment. This is due to the highly crystallinity that had the materials after freeze extraction.

- PLLA/ HAp membrane composites were fabricated and showed an increase of the storage modulus with the filler content. Particles affect PLLA thermal behavior acting as nucleating agents for PLLA crystals modifying the cold crystallisation kinetics whereas glass transition temperature of PLLA is independent of the filler content. The bioactivity is enhanced by the presence of the HAp particles more than by plasma treatment. Membranes demonstrating the faster kinetics of apatite deposition with a $\mathrm{Ca} / \mathrm{P}$ ratio similar to the physiological one are that with a $15 \%$ of HAp nanoparticles and previously exposed 
to plasma which also presented the higher value of storage modulus.

- Scaffolds filled with nanoparticles of HAp and silicate were fabricated. The increase of HAp did not permit to increase the mechanical properties even when double sonication was applied to disperse the nanofiller to the polymer solution. The combination of $9.5 \%$ of nanoparticles of HAp and $0.5 \%$ of silicate permitted to increase the mechanical properties of the medical grade scaffolds till $6.9 \pm 0.1 \mathrm{MPa}$, as double sonication permitted effective dispersion of nanofillers and silica augmented the specific surface between PLLA and nanofiller.

- The bioactivity of the scaffolds increased when incorporating only $5 \%$ of HAp and more if materials are exposed to plasma. It was possible to observe biomimetic apatite on the internal surface after 21 days in SBF. The composite material reinforced with $10 \%$ wt. of $\mathrm{HAp} / \mathrm{SiO} 2$ in proportion (95/5) showed a better bioactivity on the surface and interior after 21 days soaking in SBF.

- It was possible to fabricate an osteochondral bilayer scaffold by the combination of two scaffolds fabricated by freeze extraction and particle leaching. The two layers were joined by a thin non-porous layer to avoid blood flow from the bone to the cartilage area. The up side of the biconstruct functionalised for cartilage repair was a 1:115\%PLLA-MED scaffold, $1 \mathrm{~mm}$ high. The bottom side made with the same composition (6 $\mathrm{mm}$ high) was functionalised for good bone anchorage with a 5\% wt. content of hydroxyapatite nanoparticles. 



\section{Chapter 6. Appendix. In vitro and in vivo biological response}

In vitro and in vivo biological response of the different materials fabricated is described in this section. This study was made in collaboration with the Group of Structural Mechanics and Materials Modelling, of the University of Zaragoza and the Laboratorio de Ortopedia Experimental Área de Terapia cellular of the University of Navarra. My participation on this work consisted in the fabrication of the materials for their biological evaluation. Furthermore I add the opportunity to participate to the coordination meeting organised during the project in which each group worked on.

\subsection{IN VITRO CULTURE}

Human mesenchymal stem cells (hMSCs) were loaded into 1 and $6 \mathrm{~mm}$ scaffolds, as fabricated in section 2.1.1, p. 47, in order to establish the optimal conditions for cell seeding. Loading of scaffolds was performed as follows: for $6 \mathrm{~mm}$ thick scaffolds cells resuspended in $20 \mu \mathrm{L}$ were seeded by needle injection. Scaffolds of $1 \mathrm{~mm}$ thick were placed in a pipette tip and connected to an aspiration syringe and hMSCs were aspirated. Cells were finally allowed to attach for 3 hours and then the efficiency of seeding and the cell density achieved were used as the parameters to determine the optimal loading. Seeding efficiency was determined by quantification of DNA using spectrophotometry. Cell density was measured with DAPI and visualized using a fluorescence microscope. Cell count was performed using ImageJ software. For chondrogenic differentiation, hMSCs loaded scaffolds were maintained in culture with differentiation media for up to 21 days and then histological analysis was made. 
The seeding of hMSCs yielded a percentage of cells retained in the scaffold of $80.8 \pm 4.1$ and $71.6 \pm 9.4 \%$ for the 6 and $1 \mathrm{~mm}$ scaffolds respectively $(p>0.05)$. Cell distribution was quantified using immunofluorescence against B-actin, as the percentage of scaffold area occupied by cells (Figure 6-1B). Images are shown in Figure 6-1C. Cells were unevenly distributed in $1 \mathrm{~mm}$ scaffolds, with areas covered up to $60 \%$ with cells (center of the scaffold) with lower occupation in the outer part of the scaffold. Cells accumulated around the needle track and the center of the $6 \mathrm{~mm}$ scaffolds (Figure 6-1C); however concerning the percentage of the area occupied by cells, in $6 \mathrm{~mm}$ scaffolds it reached only $3 \%$ in those zones with higher cell concentration. Finally, SEM images obtained from the loaded scaffolds showed the presence of cells inside the PLLA (Figure 6-1D). 

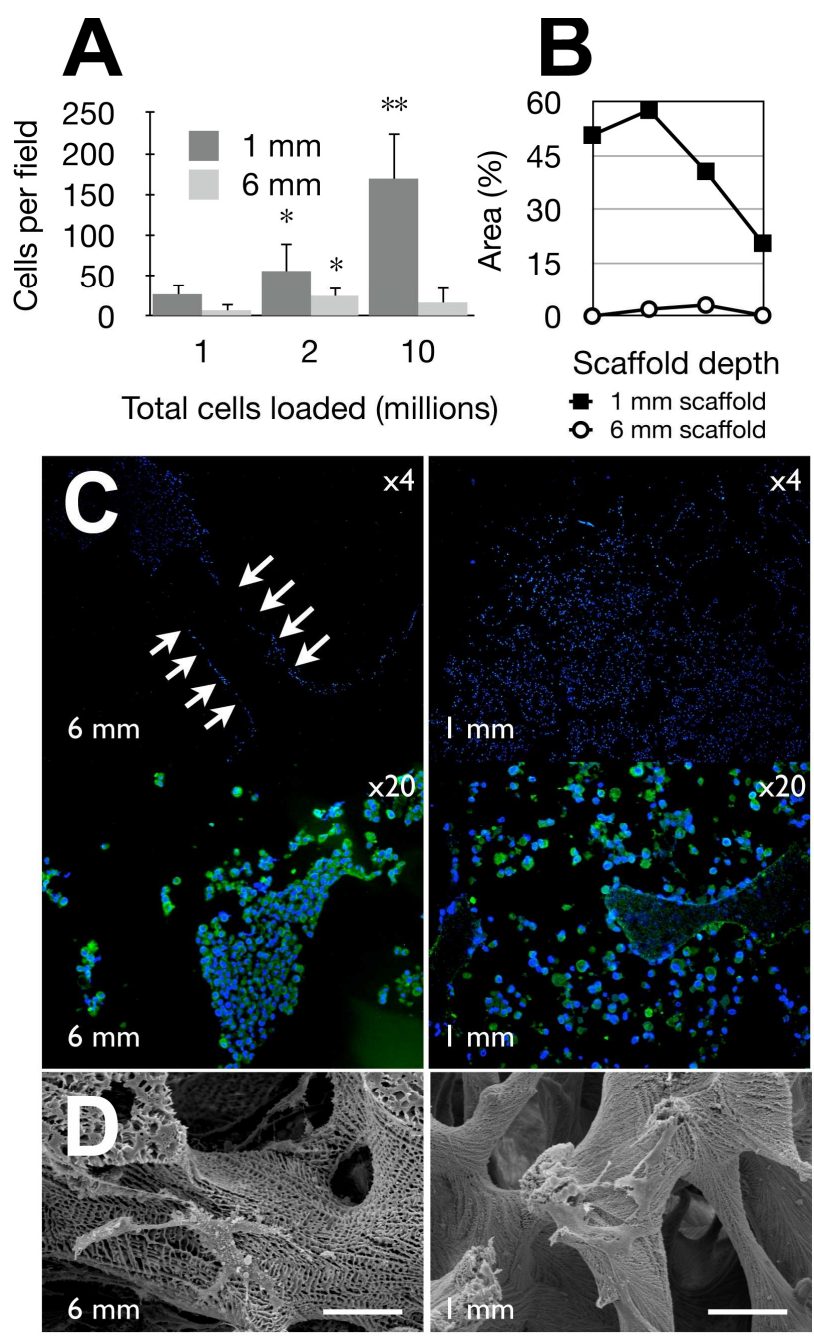

Figure 6-1: (A). Cell distribution was visualized by immunofluorescence. (B). Some representative images are shown in (C) for 6 (left panels) and $1 \mathrm{~mm}$ scaffolds (right panels). Scanning electron microscopy was performed to confirm the distribution of cells and to visualize the topography of the material (D). The arrows show the channel perforated by the syringe during the loading process. Results represent the mean $\pm S D$ of at least 3 different experiments in $A$ and $B$ and a representative study in $C$ and $D$. Scale bar: 50 $\mu \mathrm{m}$. 
Scaffolds (1 and $6 \mathrm{~mm}$ ) were maintained for 21 days in either expansion media (EM) or chondrogenic media (CM) after which cell number, spontaneous as well as induced chondrogenic differentiation were examined. The number of hMSCs in $6 \mathrm{~mm}$ scaffolds remained stable during the 21 days in expansion and chondrogenic media while there was a reduction in cell number in $1 \mathrm{~mm}$ scaffolds with EM throughout the 21 days of the experiment. When $1 \mathrm{~mm}$ scaffolds were cultured with chondrogenic medium there was a reduction similar to those treated with EM (Figure 6-2A). In both cases (EM and $\mathrm{CM}$ ), the reduction in the cell number in the scaffolds was not due to an increase in apoptosis, as demonstrated by the negative immunostaining of caspase $3 \mathrm{~A}$ (Figure 6-2B left). These results suggest that the decrease in cell number is produced by the loss of unbound cells. Finally, immunostaining with Ki67 antibody did not reveal any proliferation inside the scaffolds (Figure 6-2B). 


\section{A}
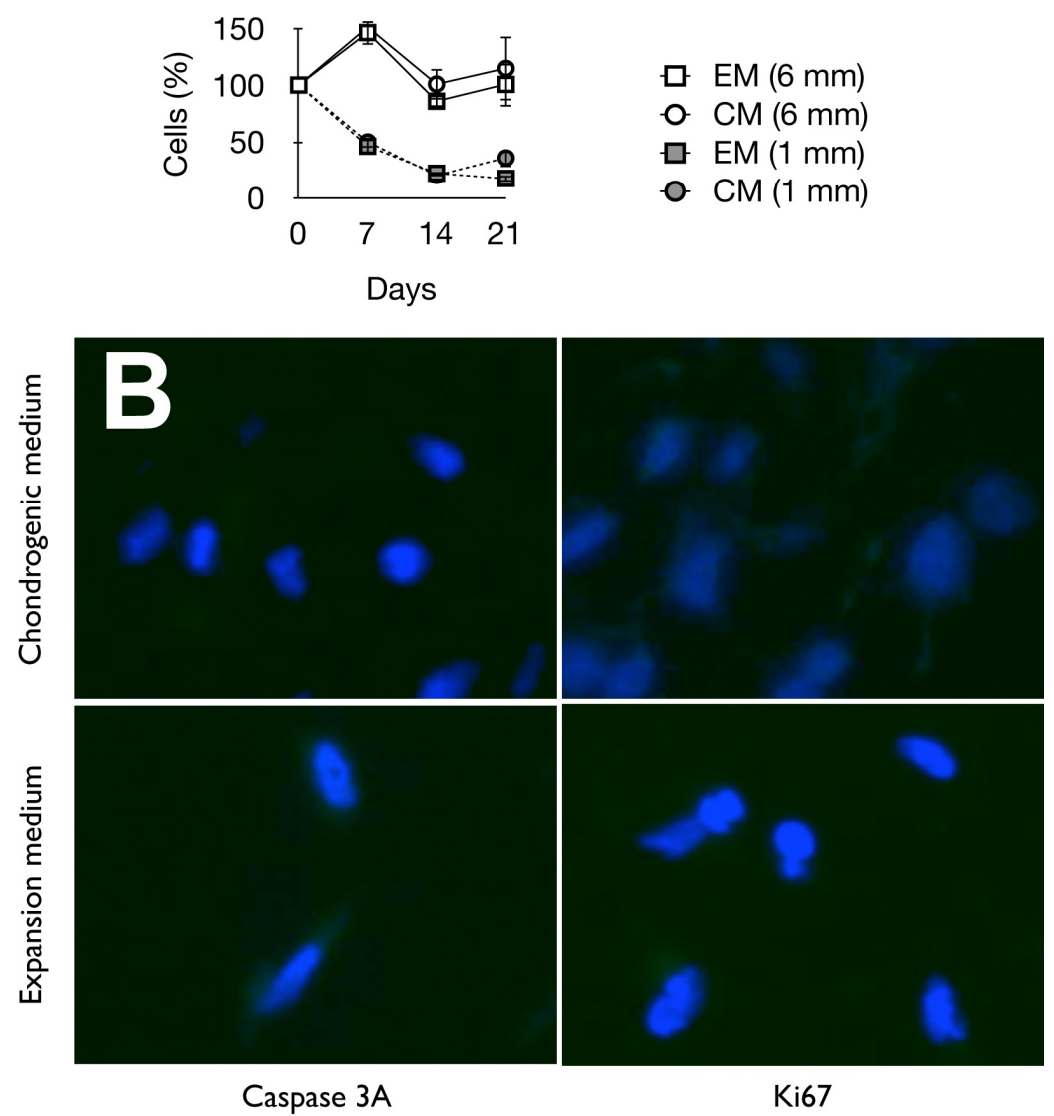

Figure 6-2: Scaffolds were seeded with hMSCs and culture for up to 21 days in EM or CM and the number of cells quantified (A). (B) Cell apoptosis and proliferation was determined by immunfluorescence staining using antibodies against caspase $3 \mathrm{~A}$ and Ki67 respectively. DAPI was used for nuclei staining. Results represent the mean \pm SD of at least 3 different experiments in $A$ and a representative study in $B$. 
The histological comparison between scaffolds maintained in expansion media showed that MSCs did not produce a significant amount of extracellular matrix components or fiber deposition either in 1 or $6 \mathrm{~mm}$ scaffolds (Figure 6-3A). However, in those scaffolds maintained in differentiation media, hMSC were surrounded by collagen fibers as demonstrated by Masson's trichrome that was particularly intense in certain areas. While cell distribution varied between 1 and $6 \mathrm{~mm}$ scaffolds, fibers were similarly distributed around the cells (Figure 6-3A).

Immunfluorescence analysis demonstrated the presence of collagen type $\mathrm{X}$ in both 1 and $6 \mathrm{~mm}$ scaffolds and to a lesser extent type I collagen. Type II collagen, specifically associated with hyaline cartilage was poorly detected in either scaffold, with some slight staining in areas with a high density of cells. Finally aggrecan was detected in both 1 and $6 \mathrm{~mm}$ scaffolds (Figure 6-3B).
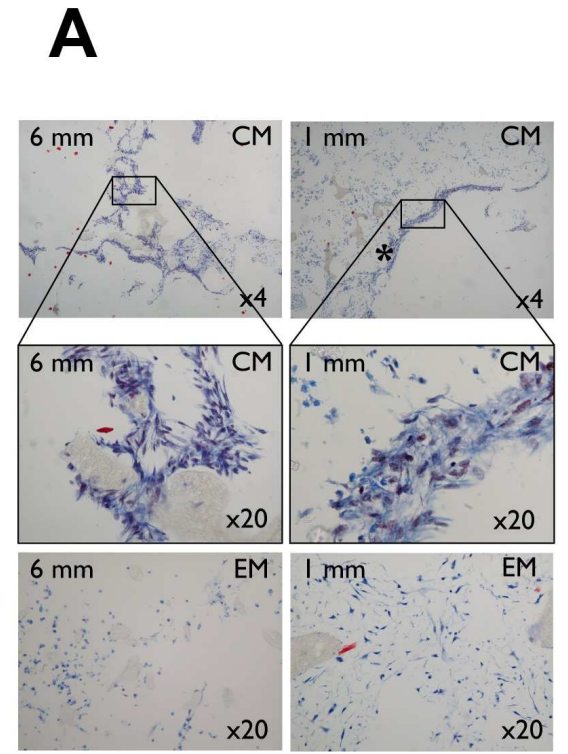


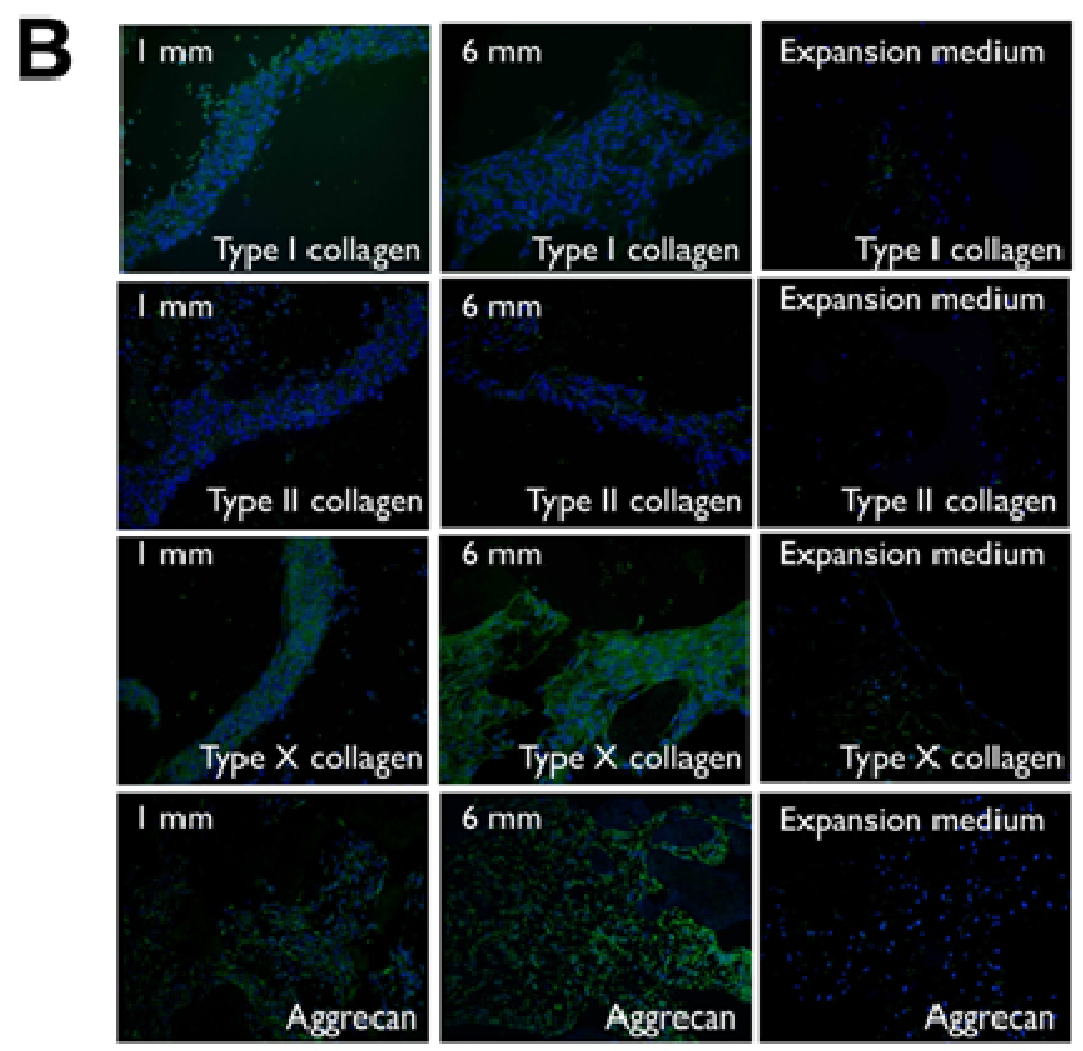

Figure 6-3: (A) Masson's trichrome staining of sections from $6 \mathrm{~mm}$ (left panels) and $1 \mathrm{~mm}$ (right panels) thick scaffolds showing the presence of matrix fibers. (B) The composition of the matrix fibers in the scaffolds after differentiation was assessed by immunofluorescence against aggrecan, and collagens type I, II and X (B). DAPI was used for nuclei staining. A representative study of at least three experiments is shown.

hMSC into the $6 \mathrm{~mm}$ thick scaffolds provided a poor distribution of cells confined in the center of the material, but with high density in the site of the injection. In the $1 \mathrm{~mm}$ thick scaffolds, the higher quantity of cells seeded achieved better distribution spreading over the scaffold, with a high density in the entire volume of the material that would eventually lead to better differentiation. 


\subsection{IMPLANTATION}

In vivo osteointegration of the scaffolds was analyzed after their implantation in the femoral condyle of 3 month aged healthy sheep. Three types of scaffolds were implanted: scaffolds only made from PLLA-MED, scaffolds filled with 5\% wt. of HAp nanoparticles and scaffolds filled with 5\%wt. of HAp and covered by a layer of biomimetic HAp after 21 days in SBF. Osteochondral lesions, $6 \mathrm{~mm}$ in diameter and $6 \mathrm{~mm}$ in depth were performed in the load bearing area of the medial femoral condyle, and scaffolds placed during the surgery time fixed by press-fit in the site of the lesions. Animals were allowed to evolve for 6 weeks and then, the complete knee joint extracted and the lesioned area isolated and subjected to histological analysis. Masson's trichrome was used for the evaluation of bone integration. Finally the study was completed with an immunofluorescence analysis for the detection of the bone molecular markers collagen type I, osteopontin and osteocalcin.

Images of Figure 6-4 show the presence of regenerated tissue inside the pores of the 3 kinds of implanted scaffold. On the first hand, PLLA with and without HAp was invaded by a fibrous-like tissue according to Masson's Trichrome staining, with abundance of collagen fiber that surround the PLLA fragments. On the other hand, the scaffold implanted with a layer of hydroxyapatite covering its pores was invaded by a tissue that showed zones with an aspect very similar to osteoids (Figure 6-4), which suggests that the regenerated tissue has characteristics of a mature bone and pointing to a more intense integration for these scaffolds.

Immunofluorescence analysis, Figure 6-4, showed differences among the materials in expression of type I collagen, a clearly higher expression of collagen $I$ is found in the scaffold covered with hydroxyapatite crystals, which could also be a 
characteristics of fibroblastic tissue and so fails to demonstrate the capacity of the material to regenerate a tissue similar to natural mature bone. The expression of both osteopontin (marker for bone remodeling) and osteocalcin (marker for bone calcification) was similar in the three kinds of scaffolds showing that processes of bone remodelling and formation occurred in the 3 scaffolds here used.
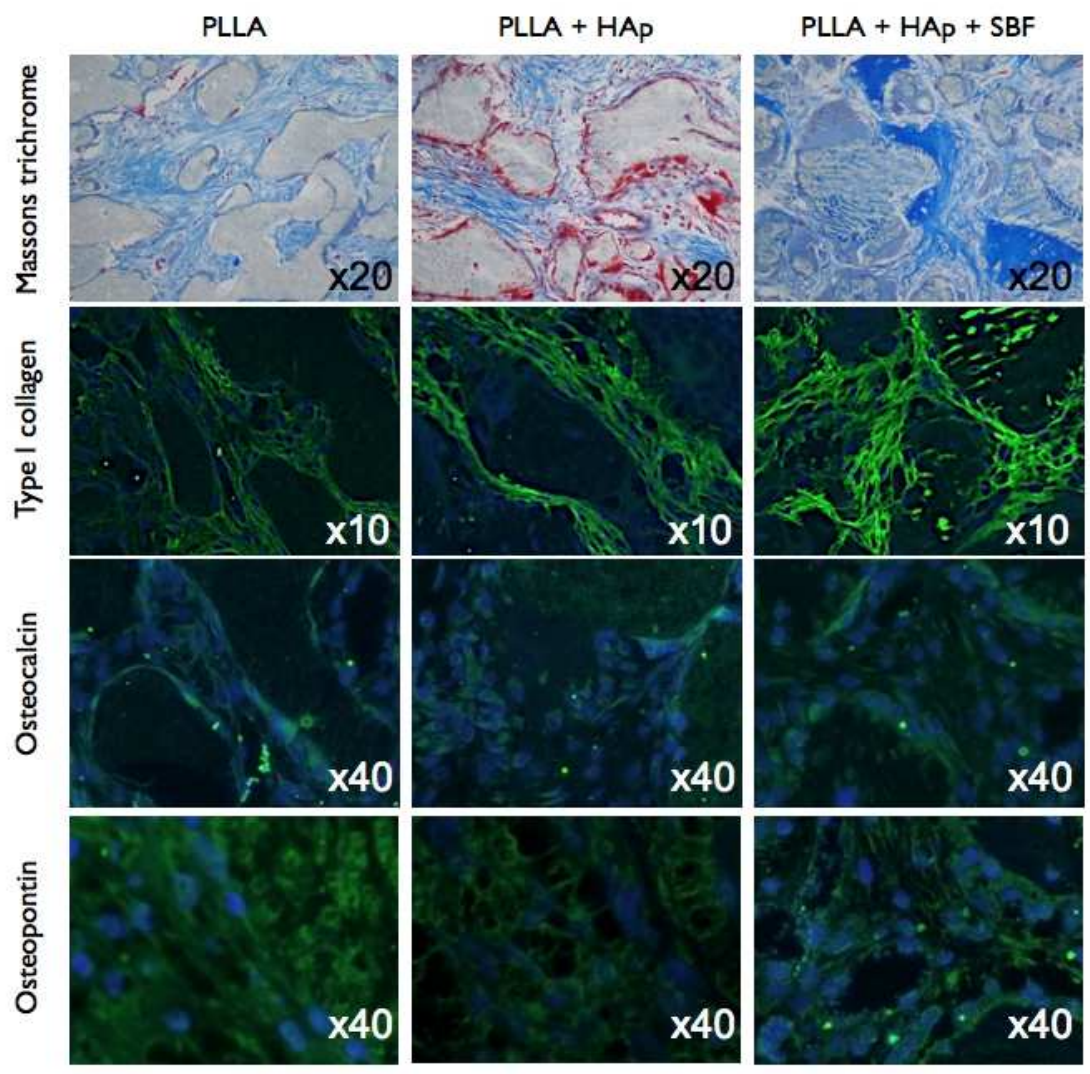

Figure 6-4: Masson's trichrome, collagen I, osteocalcine and ostepontin.

The presence of nuclei of HAp on the material's surface caused the appearance of osteoid-like structures very similar to those present in mature bone, which did not appear in the PLLA or 
PLLA/ HAp-treated lesions. The lower elastic modulus for scaffolds filled with $5 \%$ wt. nanoparticles of HAp or the scaffolds filled with nanofiller and those covered by a biomimetic layer of HAp nucleated after 21 days in SBF should be also taken into account, as a weaker material could be not adequate for implantation in bones that need mechanical resistance after the surgical procedure. The HAp nuclei formed over the HAp molecules would facilitate the differentiation of cells that penetrate inside the scaffold once in vivo allowing for a faster appearance of bone. 


\section{Glossary}

ACI

autologous Chondrocyte Implantation

BMP

bone morphogenetic proteins

CACI

collagen-covered ACI

$\chi_{\mathrm{c}}$

crystallinity

$\mathrm{CM}$

chondrogenic media

DAPI

4',6-diamidino-2-phenylindole

DCPD

dicalcium phosphateFDA

DMS

dynamic mechanical spectroscopy

DSC

differential scanning calorimetry

ECM

extra cellular matrix

EDS

elemental analysis

EM

expansion media

FDA

food and drug administration

FGF

fibroblast growth factors

FTIR

fourier transform infrared analysis

GAGs

glycosaminoglycans

GPC

gel permeation chromatography

HA

hyaluronic acid

HAp

hyadroxyapatite

HFBR

hollow fiber bioreactor

hMSC

human mesenchymal stem cells

IGF

insulin-like growth factors

MACI

hatrix-Induced autologous chondrocyte

implantation 
MSC

NBSs

$\mathrm{OA}$

$\mathrm{OCP}$

PACI

PBS

PCL

PDGF

PDLA

PDLLA

P(EMA-co-HEA)

PEMA

PEO

PGA

PLA

PLGA

PLLA

$\mathrm{SBF}$

SEM

SFF

TCP

TE

$\mathrm{Tg}$

TGF- $\beta$

THF

TGA hesenchymal stem cells

nanoparticulated bimodal porous silicas

osteoarthritis

octacalcium phosphate

periosteum ACI

phosphate bufferer saline solution

poly ( $\varepsilon$-caprolactone)

platelet-derived growth factor

poly (D-lactic acid)

poly (D, L-lactic acid)

poly(ethyl methacrylate-co-hydroxyethyl

acrylate)

poly(ethyl methacrylate)

polyethylene oxide

poly(glycolic acid)

poly (lactic acid)

poly (DL-lactic-glycolic acid)

poly (L-lactic acid)

simulated body fluid

scanning Electron Microscopy

solid free-from fabrication

tricalcium phosphate

tissue engineering

glass transition temperature

transforming growth factor beta

tetrahydrofuran

thermogravimetric analysis 
melting temperature 



\section{References}

1- B. Fawcett, in Hístología, D. W. Fawcett, Ed. (Mc Graw-Hill interamericana, Madrid, Spain, ed. 12, 2000), pp. 203.

2- J. Martel-Pelletier, C. Boileau, J. Pelletier, P. J. Roughley, "Cartilage in normal and osteoarthritis conditions," Best Practice \& Research Clinical Rheumatology 22, 351 (2008).

3- M. Guiseppe, L. Mangiavini, Deponti D, in Cartilage repair, A. Brittberg Imhoff, H. Madry, Mandelbaum B, Eds.Guildford, UK, 2010), pp. 3.

4- E. Kheir, D. Shaw, "Hyaline articular cartilage," Orthopaedics and Trauma 23, 450 (2009).

5- J. S. Temenoff, A. G. Mikos, "Review: tissue engineering for regeneration of articular cartilage," Biomaterials 21, 431 (2000).

6- J. Fritz, P. Janssen, C. Gaissmaier, B. Schewe, K. Weise, "Articular cartilage defects in the knee--Basics, therapies and results," Injury 39, 50 (2008).

7- F. Furlan, C. Galbiati,N.R. Jorgensen, J.E. Jensen, E. Mrak, A. Rubinacci, F. Talotta, P. Verde, F. Blasi, "Urokinase plasminogen activator receptor affects bone homeostasis by regulating osteoblast and osteoclast function," Journal of Bone and Mineral Research 22, 1387 (2007).

8- I. S. Byun, S. K. Sarkar, M. Anirban Jyoti M, Y.K Min, H. S. Seo, B. T. Lee, H. Y Song, "Initial biocompatibility and enhanced osteoblast response of $\mathrm{Si}$ doping in a porous BCP bone graft substitute," J. Mater. Sci. Mater. Med. 21, 1937 (2010). 
9- J. M. García, M. Doblaré, J. Cegoñino, "Bone remodelling simulation: a tool for implant design", Computational Materials Science 25, 100 (2002).

10- S. Hirose, M. Li, T. Kojima,P. H. de Freitas, S. Ubaidus, K. Oda, C. Saito, N. Amizuka, "A histological assessment on the distribution of the osteocytic lacunar canalicular system using silver staining," J. Bone Miner. Metab. 25, 374 (2007).

11- B. Clarke, "Normal Bone Anatomy and Physiology," Clin. J. Am. Soc. Nephrol. 3, S131 (2008).

12- C. Ding, P. Garnero, F. Cicuttini, F. Scott, H. Cooley, G. C. Jones, "Knee cartilage defects: association with early radiographic osteoarthritis, decreased cartilage volume, increased joint surface area and type II collagen breakdown," Osteoarthritis and Cartilage 13, 198 (2005).

13- D. Nesic, R. Whiteside, M. Brittberg, D. Wendt, I. Martin, P. D. Mainil-Varlet, "Cartilage tissue engineering for degenerative joint disease," Adv. Drug Deliv. Rev. 58, 300 (2006).

14- National institute for health and clinical excellence, "Osteoarthritis: The care and management of osteoarthritis in adults," Rep. No. guideline 59, 2008.

15- J. F. Mano, R. L. Reis, "Osteochondral defects: present situation and tissue engineering approaches," Journal of Tissue Engineering and Regenerative Medicine 1, 261 (2007).

16- M. Ochi, Y. Uchio, M. Tobita, M. Kuriwaka, "Current concepts in tissue engineering technique for repair of cartilage defect," Artif. Organs 25, 172 (2001).

17- J. M. Bert, "Role of abrasion arthroplasty and debridement in the management of osteoarthritis of the knee," Rheumatic Disease Clinics of North America 19, 725 (1993). 
18- N. Mitchell, N. Shepard,"The resurfacing of adult rabbit articular cartilage by multiple perforations through the subchondral bone," Journal of Bone and Joint Surgery - Series A 58, 230 (1976).

19- J. R. Steadman, W. G. Rodkey, J. J. Rodrigo, "Microfracture: Surgical technique and rehabilitation to treat chondral defects," Clin. Orthop., S362 (2001).

20- M. Gole, D. Poulsen, J. Marzo, Ko, Ziv I, "Chondrocyte viability in press-fit cryopreserved osteochondral allografts," Journal of Orthopaedic Research 22, 781 (2004).

21- R. S. Rohde, R. K. Studer, C. R. Chu, "Mini-pig fresh osteochondral allografts deteriorate after 1 week of cold storage," Clin. Orthop., 226 (2004).

22- L. Hangody, P. Feczkó, L. Bartha, G. Bodó, G. Kish, "Mosaicplasty for the treatment of articular defects of the knee and ankle," Clin. Orthop., S328 (2001).

23- M. Brittberg et al., "Treatment of deep cartilage defects in the knee with autologous chondrocyte transplantation," N. Engl. J. Med. 331, 889 (1994).

24- D. Grande, M. Pitman, L. Peterson, D. Menche, M. Klein, "The repair of experimentally produced defects in rabbit articular cartilage by autologous chondrocyte transplantation," Journal of Orthopaedic Research 7, 208 (1989).

25- M. Brittberg, A. Lindahl, A. Nilsson, C. Ohlsson, O. Isaksson, L. Peterson, "Treatment of deep cartilage defects in the knee with autologous chondrocyte transplantation," N. Engl. J. Med. 331, 889 (1994).

26- M. Brittberg, L. Peterson, E. Sjogren-Jansson, T. Tallheden, A. Lindahl, "Articular cartilage engineering with autologous chondrocyte transplantation: A review of recent developments," Journal of Bone and Joint Surgery - Series A 85, 109 (2003). 
27- S. O'Driscoll, F. Keeley, R. Salter, "The chondrogenic potential of free autogenous periosteal grafts for biological resurfacing of major full-thickness defects in joint surfaces under the influence of continuous passive motion. An experimental investigation in the rabbit," Journal of Bone and Joint Surgery - Series A 68, 1017 (1986).

28- H. Chiang, C. Jiang, "Repair of articular cartilage defects: Review and Perspectives," J. Formosan Med. Assoc. 108, 87 (2009).

29- J. Suh, A. årøen, T. S. Muzzonigro, M. Disilvestro, F. $\mathrm{H}$. Fu, "Injury and repair of articular cartilage: Related scientific issues," Operative Techniques in Orthopaedics 7, 270 (1997).

30- L. Peterson, T. Minas, M. Brittberg, A. Nilsson, E. Sjögren-Jansson, A. Lindahl, "Two-to 9-year outcome after autologous chondrocyte transplantation of the knee," Clin. Orthop., 212 (2000).

31- P. Cherubino, F. A. Grassi, P. Bulgheroni, M. Ronga, "Autologous chondrocyte implantation using a bilayer collagen membrane: A preliminary report," Journal of Orthopaedic Surgery 11, 10 (2003).

32- C. R. Gooding, W. Bartlett, G. Bentley, J. A. Skinner, R. Carrington, A. Flanagan, "A prospective, randomised study comparing two techniques of autologous chondrocyte implantation for osteochondral defects in the knee: Periosteum covered versus type I/III collagen covered," The Knee 13, 203 (2006).

33- S. Wakitani, A. Kawaguchi, Y. Tokuhara, K. Takaoka, "Present status of and future direction for articular cartilage repair," J. Bone Miner. Metab. 26, 115 (2008).

34- D. F. Williams, "To engineer is to create: the link between engineering and regeneration," Trends Biotechnol 24, 4 (2006). 
35- J. Ross, in Frontiers in tissue engineering, C. Patrick, A. Mikos, L. McIntire, Eds. (Elvesier Science Ltd, Oxford, U.K., 1998).

36- R. P. Lanza, R. Langer, J. Vacanti. Principles of tissue engineering. San Diego, USA: Academic Press, 1997.

37- W. Y. J. Chen, G. Abatangelo, "Functions of hyaluronan in wound repair," Wound Repair and Regeneration 7, 79 (1999).

38- S. J. Bryant, K. S. Anseth, "Hydrogel properties influence ECM production by chondrocytes photoencapsulated in poly(ethylene glycol) hydrogels," J. Biomed. Mater. Res. 59, 63 (2002).

39- R. Stoop, "Smart biomaterials for tissue engineering of cartilage," Injury 39, 77 (2008).

40- D. Eyrich, F. Brandl, B. Appel, H. Wiese, G. Maier, M. Wenzel, R. Staudenmaier, A. Goepferich, T. Blunk, "Long-term stable fibrin gels for cartilage engineering," Biomaterials 28, 55 (2007).

41- Y. Gong, Y. Zhu, Y. Liu, Z. Ma, C. Gao, J. Shen, "Layer-by-layer assembly of chondroitin sulfate and collagen on aminolyzed poly(l-lactic acid) porous scaffolds to enhance their chondrogenesis," Acta Biomaterialia 3, 677 (2007).

42- T. Sato, G. Chen, T. Ushidad, T. Ishiia, N. Ochiaia, T. Tateishib, J. Tanakab, "Evaluation of PLLA-collagen hybrid sponge as a scaffold for cartilage tissue engineering," Materials Science and Engineering: C 24, 365 (2004).

43- J. C. Schagemann et al., "The effect of scaffold composition on the early structural characteristics of chondrocytes and expression of adhesion molecules," Biomaterials 31, 2798 (2010).

44- $\quad$ H. J. Jung, K. Park, J.J. Kim, J.H. Lee, K. O. Han, D. K. Han, "Effect of RGD-immobilized dual-pore poly(Llactic acid) scaffolds on chondrocyte proliferation and 
extracellular matrix production," Artif. Organs 32, 981 (2008).

45- Z. Ma, C. Gao, Y. Gong, J. Shen, "Chondrocyte behaviors on poly--lactic acid (PLLA) membranes containing hydroxyl, amide or carboxyl groups," Biomaterials 24, 3725 (2003).

46- Z. Ma, C. Gao, Y. Gong, J. Shen, "Paraffin spheres as porogen to fabricate poly(L-lactic acid) scaffolds with improved cytocompatibility for cartilage tissue engineering," Journal of Biomedical Materials Research Part B: Applied Biomaterials 67B, 610 (2003).

47- S. H. Oh, T. H. Kim, G. I. Im, J. H. Lee, "Investigation of pore size effect on chondrogenic differentiation of adipose stem cells using a pore size gradient scaffold," Biomacromolecules 11, 1948 (2010).

48- E. Costa Martínez et al., "Effect of poly(L -lactide) surface topography on the morphology of in vitro cultured human articular chondrocytes," J. Mater. Sci. Mater. Med. 18, 1627 (2007).

49- F. Forriol, "Growth factors in cartilage and meniscus repair," Injury 40, S12 (2009).

50- A. C. Shieh, K. A. Athanasiou, "Principles of Cell Mechanics for Cartilage Tissue Engineering," Ann. Biomed. Eng. 31, 1 (2003).

51- A. H. Huang, M. J. Farrell, R. L. Mauck, "Mechanics and mechanobiology of mesenchymal stem cell-based engineered cartilage," J. Biomech. 43, 128 (2010).

52- R. Santoro, A. L. Olivares, G. Brans, D.Wirz, C. Longinotti, D. Lacroix, I. Martin,D. Wendt, "Bioreactor based engineering of large-scale human cartilage grafts for joint resurfacing," Biomaterials 31, 8946 (2010).

53- L. E. Freed et al., "Chondrogenesis in a Cell-PolymerBioreactor System," Exp. Cell Res. 240, 58 (1998).

54- E. Cimetta, E. Figallo, C. Cannizzaro, N. Elvassore, G. Vunjak-Novakovic, "Micro-bioreactor arrays for 
controlling cellular environments: Design principles for human embryonic stem cell applications," Methods 47, 81 (2009).

55- P. Habibovic, K. de Groot, "Osteoinductive biomaterials properties and relevance in bone repair," Journal of Tissue Engineering and Regenerative Medicine 1, 25 (2007).

56- H. Kagami, H. Agata, A. Tojo, "Bone marrow stromal cells (bone marrow-derived multipotent mesenchymal stromal cells) for bone tissue engineering: Basic science to clinical translation," Int. J. Biochem. Cell Biol. 43, 286 (2011).

57- M. I. Santos, K. Tuzlakoglu, S. Fuchs, M. E. Gomes, K. Peters, R. E. Unger, E. Piskin, R. L.Reis, C. J. Kirkpatrick, "Endothelial cell colonization and angiogenic potential of combined nano- and microfibrous scaffolds for bone tissue engineering," Biomaterials 29, 4306 (2008).

58- M. O Montjovent, N. Burri, S. Mark, E. Federici, C. Scaletta, P. Y. Zambelli, P. Hohlfeld, P.F.Leyvraz, L. L. Applegate, D. P. Pioletti, "Fetal bone cells for tissue engineering," Bone 35, 1323 (2004).

59- E. Monaco, M. Bionaz, S. J. Hollister, M. B. Wheeler, "Strategies for regeneration of the bone using porcine adult adipose-derived mesenchymal stem cells," Theriogenology 75, 1381 (2011).

60- T. Schubert et al., "The enhanced performance of bone allografts using osteogenic-differentiated adiposederived mesenchymal stem cells," Biomaterials 32, 8880 (2011).

61- F. J. Hua, J. D. Nam, D. Lee, "preparation of a macroporous poly(L-lactide) scaffold by liquid-liquid phase separation of a PLLA/1,4-dioxane/water ternary system in the presence of $\mathrm{NaCl}$," Macromolecular Rapid Communications 22, 1053 (2001). 
62- Y. S. Nam, T. G. Park, "Porous biodegradable polymeric scaffolds prepared by thermally induced phase separation," J. Biomed. Mater. Res. 47, 8 (1999).

63- S. J. Heo, S. E. Kim, Y. T. Hyun, D. H. Kim, H. M. Lee, Y. M. Hwang, J. W. Shin, "Biodegradable composite of poly $\varepsilon$-caprolactone/hydroxyapatite 3 -D scaffolds for bone tissue engineering," 15, 672 (2007).

64- P. Habibovic, D. C. Bassett, C. J.Doillon, C. Gerard, M. D. McKee, J. E. Barralet, "Collagen biomineralization in vivo by sustained release of inorganic phosphate ions," Adv Mater 22, 1858 (2010).

65- D. L. Nihouannen et al., "Micro-architecture of calcium phosphate granules and fibrin glue composites for bone tissue engineering," Biomaterials 27, 2716 (2006).

66- C. Wu, Y. Zhang, Y. Zhu, T. Friis, Y. Xiao, "Structureproperty relationships of silk-modified mesoporous bioglass scaffolds," Biomaterials 31, 3429 (2010).

67- C. Weinand, I. Pomerantseva, C. M. Neville, R. Gupta, E. Weinberg, I. Madisch, F. Shapiro, H. Abukawa, M. J. Troulis, J. P. Vacanti, "Hydrogel-[ß]-TCP scaffolds and stem cells for tissue engineering bone," Bone 38, 555 (2006).

68- V. Karageorgiou, D. Kaplan, "Porosity of 3D biomaterial scaffolds and osteogenesis," Biomaterials 26, 5474 (2005).

69- D. M. Cruz, M. Gomes, R. L. Reis, D. Moratal, M. Salmerón-Sánchez, J. L. Ribelles, J. F. Mano, "Differentiation of mesenchymal stem cells in chitosan scaffolds with double micro and macroporosity," Journal of Biomedical Materials Research Part A 95A, 1182 (2010).

70- K. A. Gross, L. M. L. M. Rodríguez-Lorenzo, "Biodegradable composite scaffolds with an interconnected spherical network for bone tissue engineering," Biomaterials 25, 4955 (2004). 
71- A. J. Wagoner Johnson, B. A. Herschler, "A review of the mechanical behavior of $\mathrm{CaP}$ and $\mathrm{CaP} /$ polymer composites for applications in bone replacement and repair," Acta Biomaterialia 7, 16 (2011).

72- K. Rezwan, Q. Z. Chen, J. J. Blaker, A. R. Boccaccini, "Biodegradable and bioactive porous polymer/inorganic composite scaffolds for bone tissue engineering," Biomaterials 27, 3413 (2006).

73- H. Ivankovic, G. Gallego Ferrer, E. Tkalcec, S. Orlic, M. Ivankovic. "Preparation of highly porous hydroxyapatite from cuttlefish bone", J Mater Sci Mater Med 20, 1039-1046 (2009).

74- M. M. Stevens MM. "Biomaterials for bone tissue engineering", Nature 11, 18-25 (2008).

75- A. B. Yeatts, J. P. Fisher, "Bone tissue engineering bioreactors: Dynamic culture and the influence of shear stress," Bone 48, 171 (2011).

76- G. M. Calori, D. Donati, C. D. Bella, L. Tagliabue, "Bone morphogenetic proteins and tissue engineering: future directions," Injury 40, S67 (2009).

77- P. B. Malafaya, G. A. Silva, E. T. Baran, R. L. Reis, "Drug delivery therapies I: General trends and its importance on bone tissue engineering applications," Current Opinion in Solid State and Materials Science 6, 283 (2002).

78- F. B. Basmanav, G. T. Kose, V. Hasirci, "Sequential growth factor delivery from complexed microspheres for bone tissue engineering," Biomaterials 29, 4195 (2008).

79- B. Kundu, S. C. Kundu, "Osteogenesis of human stem cells in silk biomaterial for regenerative therapy," Progress in Polymer Science 35, 1116 (2010).

80- D. Schaefera, I. Martina, P. Shastric, R.F. Paderaa, R. Langerc, L.E. Freeda, G. Vunjak-Novakovica, "In vitro generation of osteochondral composites," Biomaterials 21, 2599 (2000). 
81- S. Lien, C. Chien, T. Huang, "A novel osteochondral scaffold of ceramic-gelatin assembly for articular cartilage repair," Materials Science and Engineering: $C$ 29, 315 (2009).

82- A. Seidi, M. Ramalingam, I. Elloumi-Hannachi, S. Ostrovidov, A. Khademhosseini, "Gradient biomaterials for soft-to-hard interface tissue engineering," Acta Biomaterialia 7, 1441 (2011).

83- S. Ghosh, J. C. Viana, R. L. Reis, J. F. Mano, "Bilayered constructs based on poly(l-lactic acid) and starch for tissue engineering of osteochondral defects," Materials Science and Engineering: C 28, 80 (2008).

84- A. M. Getgood, S. J. Kew, R. Brooks, H. Aberman, T. Simon, A. K. Lynn, N. Rushton, "Evaluation of earlystage osteochondral defect repair using a biphasic scaffold based on a collagen-glycosaminoglycan biopolymer in a caprine model," The Knee In Press, Corrected Proof, - (2011).

85- L. Bi, D. Li, J. Liua, Y. Hua, P. Yanga, B. Yanga, Z. Yuan, "Fabrication and characterization of a biphasic scaffold for osteochondral tissue engineering," Mater Lett 65, 2079 (2011).

86- G. Chen, T. Sato, J. Tanaka, T. Tateishi, "Preparation of a biphasic scaffold for osteochondral tissue engineering," Materials Science and Engineering: C 26, 118 (2006).

87- W. Cui, Q. Wang, G. Chen, S. Zhou, Q. Chang, Q. Zuo, K. Ren, W. Fan, "Repair of articular cartilage defects with tissue-engineered osteochondral composites in pigs," Journal of Bioscience and Bioengineering 111, 493 (2011).

88- E. Kon, M. Delcogliano M, G. Filardo, D. Pressato, M. Busacca, B. Grigolo, G. Desando, M. Marcacci, "A novel nano-composite multi-layered biomaterial for treatment of osteochondral lesions: Technique note and 
an early stability pilot clinical trial," Injury 41, 693 (2010).

89- M. M. Stevens, "Biomaterials for bone tissue engineering," Materials Today 11, 18 (2008).

90- G. A. Silva, O. P. Coutinho, P. Ducheyne, R. L. Reis, "Materials in particulate form for tissue engineering. 2. Applications in bone," Journal of Tissue Engineering and Regenerative Medicine 1, 97 (2007).

91- S. Cho, F. Miyaji, T. Kokubo, T. Nakamura, "Induction of bioactivity of a non-bioactive glass-ceramic by a chemical treatment”, Biomaterials 18,1479 (1997).

92- T. Kokubo, "Bioactive glass ceramics: properties and applications", Biomaterials 12, 155 (1991).

93- T. Kokubo, H. Takadama, "How useful is SBF in predicting in vivo bone bioactivity?" Biomaterials 27, 2907 (2006).

94- M. Bohner, J. Lemaitre, "Can bioactivity be tested in vitro with SBF solution?" Biomaterials 30, 2175 (2009).

95- L. Müller, F. A. Müller, "Preparation of SBF with different content and its influence on the composition of biomimetic apatites," Acta Biomaterialia 2, 181 (2006).

96- Z. Hong, R. L. Reis, J. F. Mano, "Preparation and in vitro characterization of scaffolds of poly(l-lactic acid) containing bioactive glass ceramic nanoparticles," Acta Biomaterialia 4, 1297 (2008).

97- Z. Hong, R. L. Reis, J. F. Mano, "Preparation and in vitro characterization of novel bioactive glass ceramic nanoparticles," Journal of Biomedical Materials Research Part A 88A, 304 (2009).

98- T. Kokubo, H. Kim, F. Miyaji, H. Takadama, T. Miyazaki. "Ceramic/metal and ceramic/polymer composites prepared by a biomimetic process", Composites Part A: Applied Science and Manufacturing 30, 405 (1999). 
99- T. Kokubo, "Apatite formation on surfaces of ceramics, metals and polymers in body environment", Acta Materialia 46, 2519 (1998).

100- N. Olmo, A. I. Martín, A. J. Salinas, J. Turnay, M. Vallet-Regí, M. A. Lizarbe, "Bioactive sol-gel glasses with and without a hydroxycarbonate apatite layer as substrates for osteoblast cell adhesion and proliferation”, Biomaterials 24, 3383 (2003).

101- K. Zhang, Y. Wang, M. A. Hillmyer, L. F. Francis, "Processing and properties of porous poly(Llactide)/bioactive glass composites," Biomaterials 25, 2489 (2004).

102- S. Wu, H. Hsu, S. Hsu, W. Wang, W. Ho, "Preparation and characterization of four different compositions of calcium phosphate scaffolds for bone tissue engineering," Mater Charact 62, 526 (2011).

103- P. Taddei, A. Tinti, M. Reggiani, C. Fagnano, "In vitro mineralization of bioresorbable poly( $\varepsilon$-caprolactone) lapatite composites for bone tissue engineering: a vibrational and thermal investigation," J. Mol. Struct. 744-747, 135 (2005).

104- S. C. G. Leeuwenburgh, J. G. C. Wolke, M. C. Siebers, J. Schoonman, J. A. Jansen, "In vitro and in vivo reactivity of porous, electrosprayed calcium phosphate coatings," Biomaterials 27, 3368 (2006).

105- M. Lebourg, J. Antón, J. Ribelles, "Hybrid structure in PCL-HAp scaffold resulting from biomimetic apatite growth," J. Mater. Sci. Mater. Med. 21, 33 (2010).

106- A. V. Lluch, G. G. Ferrer, M. M. Pradas, "Surface modification of $\mathrm{P}(\mathrm{EMA}-\mathrm{co}-\mathrm{HEA}) / \mathrm{SiO} 2$ nanohybrids for faster hydroxyapatite deposition in simulated body fluid?", Colloids Surf B Biointerfaces 70, 218 (2009).

107- A. V. Lluch, G. G. Ferrer, M. M. Pradas, "Biomimetic apatite coating on $\mathrm{P}(\mathrm{EMA}-\mathrm{co}-\mathrm{HEA}) / \mathrm{SiO} 2$ hybrid nanocomposites", Polymer, 50, 2874 (2009). 
108- M. Lebourg, J. Antón, J. Ribelles J, "Hybrid structure in PCL-HAp scaffold resulting from biomimetic apatite growth”, J Mater Sci Mater Med 21, 33-44 (2010).

109- X. Lu, Y. Leng, "Theoretical analysis of calcium phosphate precipitation in simulated body fluid", Biomaterials, 26, 1097-1108 (2005).

110- Z. Hong, R .L. Reis, J. F. Mano,"Preparation and in vitro characterization of scaffolds of poly(L-lactic acid) containing bioactive glass ceramic nanoparticles", Acta Biomater 4, 1297 (2008).

111- V. Maquet, A. R. Boccaccini, L. Pravata, I. Notingher, R. Jérôme, "Porous poly( $\alpha$-hydroxyacid)/Bioglass composite scaffolds for bone tissue engineering. I: preparation and in vitro characterisation", Biomaterials 25, 4185 (2004).

112- Q. Fu, E. Saiz, M. N. Rahaman, A. P. Tomsia. Bioactive glass scaffolds for bone tissue engineering: state of the art and future perspectives. 2011;In Press.

113- S. C. G. Leeuwenburgh, J. G. C. Wolke, M. C. Siebers, J. Schoonman, J. A. Jansen, "In vitro and in vivo reactivity of porous, electrosprayed calcium phosphate coatings", Biomaterials 27, 3368 (2006).

114- M. Kawashita, M. Nakao, M. Minoda, H. M. Kim, T. Beppu, T. Miyamoto, T. Kokubo, T. Nakamura, "Apatite-forming ability of carboxyl group-containing polymer gels in a simulated body fluid," Biomaterials 24, 2477 (2003).

115- E. Landi, A. Tampieri, G. Celotti, R. Langenati, M. Sandri, S. Sprio, "Nucleation of biomimetic apatite in synthetic body fluids: dense and porous scaffold development," Biomaterials 26, 2835 (2005).

116- H. Kim, T. Himeno, T. Kokubo, T. Nakamura, "Process and kinetics of bonelike apatite formation on sintered hydroxyapatite in a simulated body fluid," Biomaterials 26, 4366 (2005). 
117- X. Lu, Y. Leng, "Theoretical analysis of calcium phosphate precipitation in simulated body fluid," Biomaterials 26, 1097 (2005).

118- T. Taguchi, Y. Muraoka, H. Matsuyama, A. Kishida, M. Akashi, "Apatite coating on hydrophilic polymergrafted poly(ethylene) films using an alternate soaking process," Biomaterials 22, 53 (2001).

119- A. Oyane, M. Uchida, Y. Yokoyama, C. Choong, J. Triffitt, A. Ito, "Simple surface modification of poly( $\varepsilon$ caprolactone) to induce its apatite-forming ability," Journal of Biomedical Materials Research Part A 75A, 138 (2005).

120- X. Qu, W. Cui, F. Yang, C. Min, H. Shen, J. Bei, S.Wang, "The effect of oxygen plasma pretreatment and incubation in modified simulated body fluids on the formation of bone-like apatite on poly(lactide-coglycolide) (70/30)," Biomaterials 28, 9 (2007).

121- Y. Yokoyama, A. Oyane, A. Ito, "Biomimetic coating of an apatite layer on poly(l-lactic acid); improvement of adhesive strength of the coating," J. Mater. Sci. Mater. Med. 18, 1727 (2007).

122- M. Bitou, M. Okamoto, "Fabrication of porous 3-D structure from poly(l-lactide)-based nano-composite foams. Effect of foam structure on enzymatic degradation," Polym. Degrad. Stab. 93, 1081 (2008).

123- N. Jo, T. Iwata, K. T. Lim, S. Jung, W. Lee, "Degradation behaviors of polyester monolayers at the air/water interface: Alkaline and enzymatic degradations," Polym. Degrad. Stab. 92, 1199 (2007).

124- A. C. Vieira, J. C. Vieira, J. M.Ferra, F. D. Magalhães FD, R. M. Guedes, A. T. Marques, "Mechanical study of PLA-PCL fibers during in vitro degradation," Journal of the Mechanical Behavior of Biomedical Materials 4, 451 (2011). 
125- J. S. Wiggins, M. K. Hassan, K. A. Mauritz, R. F. Storey, "Hydrolytic degradation of poly(d,l-lactide) as a function of end group: Carboxylic acid vs. hydroxyl," Polymer 47, 1960 (2006).

126- S. Li, in Scaffolding in Tissue Engineering, P.X. Ma, J. Elisseeff, Eds. (CRC Press, 2006), pp. 335-352.

127- Y. J. Wong, J. D. Bronzino. Biomaterials. Boca Raton, USA: Taylor and Francis Group, 2007. p 10-14.

128- H. Tsuji, A. Mizuno, Y. Ikada, "Properties and morphology of poly(L-lactide). III. Effects of initial crystallinity on long-term in vitro hydrolysis of high molecular weight poly(L-lactide) film in phosphatebuffered solution," J Appl Polym Sci 77, 1452 (2000).

129- H. Tsuji, Y. Ikada, "Properties and morphology of poly(-lactide) 4. Effects of structural parameters on long-term hydrolysis of poly(-lactide) in phosphatebuffered solution," Polym. Degrad. Stab. 67, 179 (2000).

130- H. Tsuji, Y. Ikada, "Properties and morphology of poly(L-lactide). II. Hydrolysis in alkaline solution," Journal of Polymer Science Part A: Polymer Chemistry 36, 59 (1998).

131- H. Tsuji, K. Nakahara, "Poly(L-lactide). IX. Hydrolysis in acid media," J Appl Polym Sci 86, 186 (2002).

132- K. H. Lam, P. Nieuwenhuis, I. Molenaar, H. Esselbrugge, J. Feijen, P. J. Dijkstra, J. M. Schakenraad K. H, "Biodegradation of porous versus non-porous poly(L-lactic acid) films," J. Mater. Sci. Mater. Med. 5, 181 (1994).

133- D. Garlotta, "A Literature Review of Poly(Lactic Acid)," Journal of Polymers and the Environment 9, 63 (2001).

134- L. . Lim, R. Auras, M. Rubino, "Processing technologies for poly(lactic acid)," Progress in Polymer Science 33, 820 (2008). 
135- A. Södergård, M. Stolt, "Properties of lactic acid based polymers and their correlation with composition," Progress in Polymer Science 27, 1123 (2002).

136- M. Yasuniwa, S. Tsubakihara, K. Iura, Y. Ono, Y. Dan, K. Takahashi, "Crystallization behavior of poly(l-lactic acid)," Polymer 47, 7554 (2006).

137- M. Yasuniwa, K. Sakamo, Y. Ono, W. Kawahara, "Melting behavior of poly(l-lactic acid): X-ray and DSC analyses of the melting process," Polymer 49, 1943 (2008).

138- $\quad$ Y. Wang, J. F. Mano, "Effect of structural relaxation at physiological temperature on the mechanical property of poly(L-lactic acid) studied by microhardness measurements," J Appl Polym Sci 100, 2628 (2006).

139- Y. Wang, J. L. Gómez Ribelles, M. Salmerón Sánchez, J. F. Mano, "Morphological Contributions to Glass Transition in Poly(l-lactic acid)," Macromolecules 38, 4712 (2005).

140- I. Armentano, M. Dottori, E. Fortunati, S. Mattioli, J. M. Kenny, "Biodegradable polymer matrix nanocomposites for tissue engineering: A review," Polym. Degrad. Stab. 95, 2126 (2010).

141- G. Chen, T. Ushida, T. Tateishi, "Development of biodegradable porous scaffolds for tissue engineering," Materials Science and Engineering: C 17, 63 (2001).

142- C. Liu, Z. Xia, J. T. Czernuszka, "Design and Development of Three-Dimensional Scaffolds for Tissue Engineering," Chem. Eng. Res. Design 85, 1051 (2007).

143- P. Mäkelä, T. Pohjonen, P. Törmälä, T. Waris, N. Ashammakhi, "Strength retention properties of selfreinforced poly -lactide (SR-PLLA) sutures compared with polyglyconate (MaxonR) and polydioxanone (PDS) sutures. An in vitro study," Biomaterials 23, 2587 (2002). 
144- $\quad$ B. Duan, M. Wang, W. Y. Zhou, W. L. Cheung, Z. Y. Li, W. W. Lu, "Three-dimensional nanocomposite scaffolds fabricated via selective laser sintering for bone tissue engineering," Acta Biomaterialia 6, 4495 (2010).

145- K. F. Leong, C. M. Cheah, C. K. Chua, "Solid freeform fabrication of three-dimensional scaffolds for engineering replacement tissues and organs," Biomaterials 24, 2363 (2003).

146- J. M. Taboas, R. D. Maddox, P. H. Krebsbach, S. J. Hollister, "Indirect solid free form fabrication of local and global porous, biomimetic and composite 3D polymer-ceramic scaffolds," Biomaterials 24, 181 (2003).

147- K. T. Kurpinski, J. T. Stephenson, R. R. R. Janairo, H. Lee, S. Li, "The effect of fiber alignment and heparin coating on cell infiltration into nanofibrous PLLA scaffolds," Biomaterials 31, 3536 (2010).

148- J. Li, Y. Li, L. Li, A. F.T. Mak, F. Ko, L. Qin, "Preparation and biodegradation of electrospun PLLA/keratin nonwoven fibrous membrane," Polym. Degrad. Stab. 94, 1800 (2009).

149- X. Zhang, M. Kotaki, S. Okubayashi, S. Sukigara, "Effect of electron beam irradiation on the structure and properties of electrospun PLLA and PLLA/PDLA blend nanofibers," Acta Biomaterialia 6, 123 (2010).

150- J. Lannutti, D. Reneker, T. Ma, D. Tomasko, D. Farson, "Electrospinning for tissue engineering scaffolds," Materials Science and Engineering: C 27, 504 (2007).

151- L. Wu, D. Jing, J. Ding, "A room-temperature injection molding/particulate leaching approach for fabrication of biodegradable three-dimensional porous scaffolds," Biomaterials 27, 185 (2006).

152- S. Ghosh, J. C. Viana, R. L. Reis, J. F. Mano, "Development of porous lamellar poly(l-lactic acid) 
scaffolds by conventional injection molding process," Acta Biomaterialia 4, 887 (2008).

153- L. Budyanto, Y. Goh, C. Ooi, "Fabrication of porous poly(L-lactide) (PLLA) scaffolds for tissue engineering using liquid-liquid phase separation and freeze extraction," J. Mater. Sci. Mater. Med. 20, 105 (2009).

154- J. Kim, K. Taki, S. Nagamine, M. Ohshima, "Preparation of poly(L-lactic acid) honeycomb monolith structure by unidirectional freezing and freeze-drying," Chemical Engineering Science 63, 3858 (2008).

155- M. Lebourg, J. S. Antón, J. L. G. Ribelles, "Porous membranes of PLLA-PCL blend for tissue engineering applications," European Polymer Journal 44, 2207 (2008).

156- M. H. Ho, P. Y. Kuo, H. J. Hsieh, T. Y. Hsien,L. T. Hou, J. Y. Lai ,D. M. Wang, "Preparation of porous scaffolds by using freeze-extraction and freeze-gelation methods," Biomaterials 25, 129 (2004).

157- C. Schugens, V. Maquet, C. Grandfils, R. Jérôme, P. Teyssie, "Biodegradable and macroporous polylactide implants for cell transplantation: 1. Preparation of macroporous polylactide supports by solid-liquid phase separation”, Polymer 37, 1027-1038 (1996).

158- J. Chen, S. Tu, R. Tsay, "A morphological study of porous polylactide scaffolds prepared by thermally induced phase separation", Journal of the Taiwan Institute of Chemical Engineers 41, 229 (2010).

159- Y. Kang, G. Yin, Q. Yuan, Y. Yao, Z. Huang, X. Liao, B. Yang, L. Liao, H. Wang, "Preparation of poly(l-lactic acid)/[ß]-tricalcium phosphate scaffold for bone tissue engineering without organic solvent," Mater Lett 62, 2029 (2008).

160- C. R. Kothapalli, M. T. Shaw, M. Wei, "Biodegradable HA-PLA 3-D porous scaffolds: Effect of nano-sized filler 
content on scaffold properties," Acta Biomaterialia 1, 653 (2005).

161- G. Wei, P. X. Ma, "Structure and properties of nanohydroxyapatite/polymer composite scaffolds for bone tissue engineering," Biomaterials 25, 4749 (2004).

162- X. Wang, G. Song, T. Lou, "Fabrication and characterization of nano-composite scaffold of PLLA/silane modified hydroxyapatite," Med. Eng. Phys. 32, 391 (2010).

163- C. Yang, K. Cheng, W. Weng, C. Yang, "OTS-modified HA and its toughening effect on PLLA/HA porous composite," J. Mater. Sci. Mater. Med. 20, 667 (2009).

164- J. El Haskouri, J. M. Morales, D. Ortiz de Zárate, L. Fernández, J. Latorre, C. Guillem, A. Beltrán, D. Beltrán, P. Amorós, "Nanoparticulated silicas with bimodal porosity: chemical control of the pore sizes," Inorg. Chem. 47, 8267 (2008).

165- V. A. Acosta S., PhD thesis, "Ingeniería de tejidos del cartilage articular: caracterización y modelado del comportamiento mecánico", Universidad de Zaragoza (2011).

166- L. Gibson, M. Ashby, in Cellular solids, Structure and properties. (Cambridge University Press, ed. second, 1997), pp. 175, chap. 5.

167- C. Delabarde, C. J. G. Plummer, P. Bourban, J. E. Månson, "Accelerated ageing and degradation in poly-llactide/hydroxyapatite nanocomposites," Polym. Degrad. Stab. 96, 595 (2011).

168- L. He, Yanqing Zhang, X. Zeng, D. Quan, S. Liao, Y. Zeng, J. Lu, S. Ramakrishna, "Fabrication and characterization of poly(l-lactic acid) 3D nanofibrous scaffolds with controlled architecture by liquid-liquid phase separation from a ternary polymer-solvent system," Polymer 50, 4128 (2009). 
169- E. Meaurio, E. Zuza, J. Sarasua, "Miscibility and Specific Interactions in Blends of Poly(l-Lactide) with Poly(Vinylphenol)," Macromolecules 38, 1207 (2005).

170- P. van de Witte, P. J. Dijkstra, J. W. A. B. v. den, J. Feijen, "Phase behavior of polylactides in solventnonsolvent mixtures," J. Polym. Sci. Part B 34, 2553 (1996).

171- H. Sawalha, K. Schroën, R. Boom, "Polylactide films formed by immersion precipitation: Effects of additives, nonsolvent, and temperature," J Appl Polym Sci 104, 959 (2007).

172- H. Li, M. A. Huneault, "Effect of nucleation and plasticization on the crystallization of poly(lactic acid)," Polymer 48, 6855 (2007).

173- O. Persenaire, M. Alexandre, P. Degée, P. Dubois, "Mechanisms and Kinetics of Thermal Degradation of Poly(E-caprolactone)," Biomacromolecules 2, 288 (2001).

174- C. Chen, J. Chueh, H. Tseng, H. Huang, S. Lee, "Preparation and characterization of biodegradable PLA polymeric blends," Biomaterials 24, 1167 (2003).

175- Y. Gong, Q. Zhou, C. Gao, J. Shen, "In vitro and in vivo degradability and cytocompatibility of poly(l-lactic acid) scaffold fabricated by a gelatin particle leaching method," Acta Biomaterialia 3, 531 (2007).

176- Y. Kang, G. Yin, L. Luo, K. Wang, Y. Zhang, "Effects of mechanical stress on the in vitro degradation of porous composite scaffolds for bone tissue engineering," Key Engineering Materials 342-343, 273 (2007).

177- M. Lebourg., $\mathrm{PhD}$ thesis, "soportes macroporosos biodegradables basados en la poicaproplactona para la ingeniería tisular", Universitat Politècnica de València (2008).

178- A. V. Lluch, G. G. Ferrer, M. M. Pradas, "Surface modification of $\mathrm{P}(\mathrm{EMA}-\mathrm{co}-\mathrm{HEA}) / \mathrm{SiO} 2$ nanohybrids for faster hydroxyapatite deposition in simulated body 
fluid?" Colloids and Surfaces B: Biointerfaces 70, 218 (2009).

179- H. Deplaine, J. L. G. Ribelles, G. G. Ferrer, "Effect of the content of hydroxyapatite nanoparticles on the properties and bioactivity of poly(l-lactide) - Hybrid membranes," Composites Sci. Technol. 70, 1805 (2010).

180- H. Tsuji, K. Ikarashi, "In vitro hydrolysis of poly(lactide) crystalline residues as extended-chain crystallites. Part I: long-term hydrolysis in phosphatebuffered solution at $37^{\circ} \mathrm{C}, "$ Biomaterials 25, 5449 (2004).

181- Y. Kang, X. Xu, G. Yin, A. Chen, L. Liao, Y. Yao, Z. Huang, X. Liao, "A comparative study of the in vitro degradation of poly(l-lactic acid)/B-tricalcium phosphate scaffold in static and dynamic simulated body fluid," European Polymer Journal 43, 1768 (2007).

182- S. Koutsopoulos, "Synthesis and characterization of hydroxyapatite crystals: A review study on the analytical methods," J. Biomed. Mater. Res. 62, 600 (2002).

183- J. Li, D. Zhu, J. Yin, Y. Liu, F. Yao, K. Yao, "Formation of nano-hydroxyapatite crystal in situ in chitosanpectin polyelectrolyte complex network," Materials Science and Engineering: C 30, 795 (2010).

184- M. Supová, "Problem of hydroxyapatite dispersion in polymer matrices: a review," J. Mater. Sci. Mater. Med. 20, 1201 (2009).

185- S. Yan, J. Yin, Y. Yang, Z. Dai, J. Ma, X. Chen, "Surface-grafted silica linked with l-lactic acid oligomer: A novel nanofiller to improve the performance of biodegradable poly(l-lactide)," Polymer 48, 1688 (2007). 